\title{
Forced Response and Stability of Flexible Rotor-Bearing Systems with Squeeze Film Dampers
}

\author{
A Thesis \\ Presented to
}

the Faculty of the School of Engineering and Applied Science

University of Virginia

\begin{abstract}
In Partial Fulfillment
of the requirements for the Degree

Doctor of Philosophy (Mechanical and Aerospace Engineering)

by
\end{abstract}

Feng He

December 2013 
(c) 2013 Feng He 


\section{Approval Sheet}

This thesis is submitted in partial fulfillment of the requirements for the degree of

Doctor of Philosophy (Mechanical and Aerospace Engineering)

Feng He

This thesis has been read and approved by the Examining Committee:

Houston Wood, Co-Advisor

Robert Rockwell, Co-Advisor

Richard Kent, Chairman

Zongli Lin

Robert Ribando

Roger Fittro

Accepted for the School of Engineering and Applied Science:

James H. Aylor, Dean

December 2013 


\section{Abstract}

Since 1960s, Squeeze Film Dampers (SFDs) have been widely applied to rotating machinery, including ground-based turbines, compressors and aero-engines. The reason behind the popularity of SFDs is their ability to attenuate system vibrations and improve system stability. Many efforts have been made to understand their properties at both component and system level. However, the discrepancies between predictions and experiments and the deficiencies of theoretical models to satisfy industrial requirements still exist in those studies.

For the analysis of SFDs at component level, the major assumptions in the previous studies include open-end with full leakage, perfect seals with no leakage and ideal supply/discharge conditions. These presumptions fail to give reliable predictions of damping capacities in industrial applications. Another limitation of the component level analysis in the previous work is the constraint on the journal motion, which requires the journal to precess synchronously within either circular centered orbits or off-centered circular orbits having small amplitude. These kinds of presumptions prevent accurate predictions for the performances of SFDs in practical rotating systems.

Besides the limitations of the component level analysis, the methods for predictions at the system level fail to handle complicated operating conditions for the practical rotating machinery with SFDs. The force coefficient method using traditional iteration procedures is sensitive to the variation of operating conditions and can not deal with rotating systems having excessive dynamic loads. Additionally, when using the direct force methods, most of the past studies consider simple rotating systems on SFDs, and all of them apply extensively 
the full end-leakage model.

To meet the needs of industrial applications with SFDs, this work focuses on considering realistic damper configurations and improving the efficiency and robustness of prediction tools for rotordynamic analysis. One of the common sealing conditions, the piston ring seals, is investigated with two different supply/discharge methods, the central groove and direct holes. The finite difference method is used to obtain the pressure distribution and damping capacity of these complicated dampers. To incorporate these kinds of dampers into the rotordynamic analysis of complex rotating systems, this work also utilizes the ideal of homotopy and predictor-corrector procedure to improve the traditional methods for the analysis at system level. The parametric studies on a flexible rotor-bearing system and an aero-engine are discussed using the developed methods.

The results of this study show that SFDs with piston ring seals can generate three times more damping than that of open-end SFDs. Different from the analysis using simplified models, the current work finds that the pressure within the supply groove is not always zero, but dependent on the groove depth. The influence of supply pressure, which cannot be captured by the simplified damper models, is observed to increase the effective damping and improve system stability. The developed curve intersection method enables effective parametric study on flexible rotor-bearing systems with excessive unbalance forces. The improved harmonic balance method results in more than five times faster computation speed than the nonlinear transient analysis in obtaining steady state responses. The application of the developed methods to the aero-engine shows the effects of dampers with practical configurations on the rotordynamics of complicated systems for the first time. The simplified damper model is found to under predict the responses about 30\% than these from the finite difference model. Additionally, the prediction methods and the results from current work pave the base for the optimized design of industrial rotating systems on SFDs with complex configurations. 


\section{Acknowledgements}

First and foremost, I am deeply grateful to the Rotating Machinery and Controls (ROMAC) laboratory for the support throughout this process. I have learned a lot from this great opportunity that was given to me.

I would like to thank my advisors, Houston Wood and Robert Rockwell, for their support and help through this work. Thanks also to Richard Kent, Zongli Lin, Robert Ribando and Roger Fittro for serving on my examination committee and giving valuable suggestions and critique of this work.

I would like to thank Paul Allaire for guiding me into the interesting world of rotordynamics and advising for the first couple years. I would also like to thank Tim Dimond for his suggestions at the beginning of this thesis.

I appreciate all of my friends and colleagues Amir Younan, Weimin Wang, Wei Jiang, Jin Zhou, Jason Kaplan, Jianmin Cao, Saeid Dousti and all other ROMAC people who have been very helpful for the discussions throughout my Ph.D work.

My parents deserve so much appreciation for all the love and supports they gave me through my life. Finally, I must thank my wife, Gu Lin, for her enduring support. 


\section{Contents}

Acknowledgements $\quad$ iv

Contents

List of Figures . . . . . . . . . . . . . . . . . . . . . xi

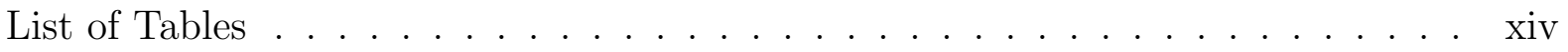

1 Problem Statement $\quad 1$

1.1 Introduction . . . . . . . . . . . . . . . . . . 1

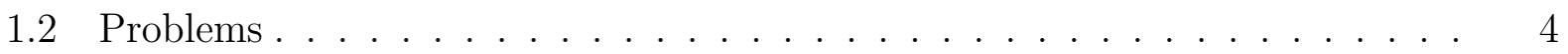

1.2.1 Component Level . . . . . . . . . . . . . . . . . . . . . . . . . . . . . . . . . . 4

1.2 .2 System Level . . . . . . . . . . . . . . . . . . . . . . . . . . . 9

1.3 Dissertation Outline . . . . . . . . . . . . . . . . . 11

2 Rotor-Bearing Systems with SFDs: Review 15

2.1 Lubricant Properties . . . . . . . . . . . . . . . . . . . . . . . 15

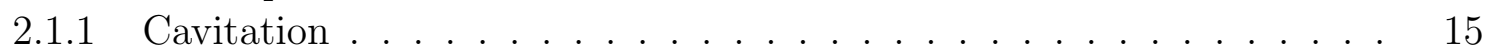

2.1 .2 Fluid Inertia . . . . . . . . . . . . . . . . . . . . . . . . . . . . . . . . . . . . .

2.2 Sealing Condition . . . . . . . . . . . . . . . . . . . . 19

2.2 .1 O-rings . . . . . . . . . . . . . . . . . . . . . . . . . . . . . . . . . 19

2.2 .2 Piston Rings . . . . . . . . . . . . . . . . . . . . . . . . . . . 19

2.3 Supply and Discharge Condition . . . . . . . . . . . . . . . . . . . . 20

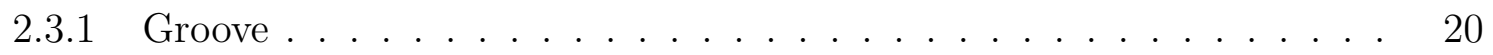

2.3 .2 Orifice Hole . . . . . . . . . . . . . . . . . . . . . . . 21

2.4 Forced Response . . . . . . . . . . . . . . . . . . . . . 22

2.4.1 Force Coefficients Method . . . . . . . . . . . . . . . . . 22

2.4 .2 Direct Force Method . . . . . . . . . . . . . . . . . . . 23

2.5 Stability Analysis . . . . . . . . . . . . . . . . . . . . . . . . . . . . . . . . . . . . .

2.5.1 Force Coefficients Method . . . . . . . . . . . . . . . 27

2.5.2 Direct Force Method . . . . . . . . . . . . . . . . . 27

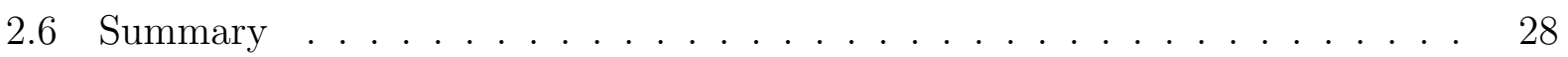

3 SFDs with End Seals and Supply/Discharge Conditions 30

3.1 Piston Rings . . . . . . . . . . . . . . . . . . . . . 31

3.1.1 Pressure Distribution Using FDM . . . . . . . . . . . . . . . . . . 32

3.1.2 Force Coefficients of SFDs with Piston Ring Seals . . . . . . . . . . . 38 
3.2 Hole Supply/Discharge . . . . . . . . . . . . . . . . . . . . . . . . . 45

3.2 .1 Pressure Distribution . . . . . . . . . . . . . . . . . . 45

3.2.2 Force Coefficients of SFDs with Hole Supply/Discharge . . . . . . . . 47

3.3 Groove Supply/Discharge . . . . . . . . . . . . . . . . . . . . 54

3.3 .1 Pressure Distribution . . . . . . . . . . . . . . . . . 54

3.3 .2 Force Coefficients of SFDs with Groove . . . . . . . . . . . . 58

3.4 Summary . . . . . . . . . . . . . . . . . . . . . . . . . 64

4 Rotordynamics of Flexible Rotor Systems on SFDs using Force Coefficients $\quad 66$

4.1 Force Coefficient Model of SFDs . . . . . . . . . . . . . . . . . . . . 67

4.1 .1 Open End(Full Leakage) . . . . . . . . . . . . . . . . . . . . . . 69

4.1.2 Central Groove Supply/Discharge with Full or No Leakage . . . . . . 70

4.1 .3 Open End SFDs with Inertia Effects . . . . . . . . . . . . . 71

4.2 Curve Intersection Method . . . . . . . . . . . . . . . . . . . . . . 72

4.3 Incorporate SFDs Using Force Coefficients . . . . . . . . . . . . . . 75

4.4 Unbalance Responses Using Force Coefficients . . . . . . . . . . . . . . 77

4.4.1 Mathematical Model for Unbalance Responses with SFDs . . . . . . . 78

4.4.2 Responses with Varying Operating Conditions of the Rotor . . . . . . 79

4.4.3 Responses with Varying Parameters of the SFD . . . . . . . . . . 84

4.5 Stability Analysis Using Force Coefficients . . . . . . . . . . . . . . . 88

4.5.1 Mathematical Model for Stability Analysis with SFDs . . . . . . . . 88

4.5.2 Stability with Varying Operating Conditions of the Rotor . . . . . . . 90

4.5.3 Stability with Varying Parameters of the SFD . . . . . . . . . . . 92

4.6 Summary . . . . . . . . . . . . . . . . . . . . . . . . 94

5 Rotordynamics of Flexible Rotor Systems on SFDs using Direct Force $\quad 95$

5.1 The Harmonic Balance Method . . . . . . . . . . . . . . . . . . . . 97

5.1.1 Forced Responses Using Harmonic Balance Method . . . . . . . . . . 98

5.1.2 Stability Analysis Using Harmonic Balance Method . . . . . . . . . . 100

5.2 Predictor-Corrector Procedure . . . . . . . . . . . . . . . . . . . . . 102

5.2 .1 Homotopy . . . . . . . . . . . . . . . . . . . . 103

5.2 .2 Predictors . . . . . . . . . . . . . . . . . . . . . . 105

5.2 .3 Correctors . . . . . . . . . . . . . . . . . . . 106

5.3 Application to a Flexible Rotor-Bearing System . . . . . . . . . . . . . 107

5.3.1 Responses with Varying Unbalance . . . . . . . . . . . . . . . 108

5.3 .2 Responses with Unidirectional Loads . . . . . . . . . . . . . . . . 112

5.3.3 Responses with Varying Stiffness of Centering Spring . . . . . . . . 115

5.3.4 Responses with Effects of Gyroscopics . . . . . . . . . . . . 118

5.3.5 Comparison with Force Coefficient Method . . . . . . . . . . . . . . . 121

5.4 Summary . . . . . . . . . . . . . . . . . . . . . . . 123 
6 Rotordynamic Analysis of an Aero-Engine $\quad 125$

6.1 Forced Responses of the Low Pressure Rotor . . . . . . . . . . . . . . . . . . 127

6.1.1 Comparison with the Transient Analysis . . . . . . . . . . . . . . 128

6.1.2 Responses with Varying Unbalance . . . . . . . . . . . . . . . . . . . 131

6.1.3 Responses with Varying Spring Stiffness . . . . . . . . . . . . . . . . 134

6.2 Rotordynamics of the High Pressure Rotor . . . . . . . . . . . . . . 137

6.2.1 Rotordynamics with Piston Ring Seals - a Simplified Model . . . . . 138

6.2.2 Piston-Ring Seals with Groove Supply/Discharge . . . . . . . . . . . 151

6.2.3 Piston-Ring Seals with Direct Hole Supply/Discharge . . . . . . . . . 156

6.3 Summary . . . . . . . . . . . . . . . . . . 158

7 Conclusions and Recommendations $\quad 160$

7.1 Conclusions . . . . . . . . . . . . . . . . . . . . . . 160

7.2 Contributions . . . . . . . . . . . . . . . . . . . . . . 168

7.3 Recommendations for Future Work . . . . . . . . . . . . . . . . 170

$\begin{array}{ll}\text { References } & 172\end{array}$

Appendix A: Forced Responses Using Harmonic Balance Method 182

Appendix B: Stability Using Harmonic Balance Method 186 


\section{Nomenclature}

$\Delta X \quad$ Mesh size in circumferential direction

$\Delta Z \quad$ Mesh size in axial direction

$\widetilde{\mathbf{F}}(\mathbf{X})_{\text {constant }}$ Constant term of Fourier coefficient for external forces

$\widetilde{\mathbf{F}}(\mathbf{X})_{\cos }$ Cosine term of Fourier coefficient for external forces

$\widetilde{\mathbf{F}}(\mathbf{X})_{\sin }$ Sine term of Fourier coefficient for external forces

$\{\mathbf{f}\} \quad$ External load vector

$\{\mathbf{x}\} \quad$ Displacement vector for all the nodes from finite element beam model

C Global damping matrix, including the damping from SFDs

G Global gyroscopic matrix

K Global stiffness matrix, including the effectiveness stiffness from SFDs

M Global mass matrix, containing the mass of the journal

$\mu \quad$ Dynamic viscosity of lubricant

$\Omega \quad$ Rotating speed of the shaft

$\omega \quad$ Precession speed of the journal

$\omega_{i} \quad i t h$ harmonic component presented in the steady state responses 
$\overline{C_{r t}} \quad$ Dimensionless cross-coupled damping in polar coordinate

$\overline{C_{t t}} \quad$ Dimensionless direct damping in polar coordinate

$\Phi \quad$ Circumferential direction in global coordinate

$\rho \quad$ Density of lubricant

$\theta_{i, j} \quad$ Angle of mesh point $(\mathrm{i}, \mathrm{j})$

$\varphi \quad$ Attitude angle

$a_{r} \quad$ Acceleration of the journal in radial direction

C Mesh size in circumferential direction

$C_{h} \quad$ Flow coefficient related to flow path properties of hole supply/discharge

$C_{p} \quad$ Leakage coefficient

$C_{r t} \quad$ Cross coupled damping in polar coordinate

$C_{t t} \quad$ Direct damping in polar coordinate

$D \quad$ Diameter of the journal

$e \quad$ Eccentricity of the journal

$F_{r} \quad$ Damper force in radial direction within the polar coordinate

$F_{t} \quad$ Damper force in tangential direction within the polar coordinate

$F_{x} \quad$ Damper force in $\mathrm{x}$ direction within the Cartesian coordinate

$F_{y} \quad$ Damper force in y direction within the Cartesian coordinate

$h \quad$ Film thickness of the film land

$h_{s} \quad$ Radial clearance between piston ring and housing 
$k \quad$ total number of frequencies used in harmonic balance method, integer

$L \quad$ Effective axial length of the film land

$M_{r r} \quad$ Direct mass terms from fluid inertia effect

$M_{t r} \quad$ Cross-coupled mass terms from fluid inertia effect

$p \quad$ The pressure inside the damper

$P_{a} \quad$ Ambient pressure

$p_{s} \quad$ Supply pressure

$R \quad$ Radius of the journal

Re $\omega C^{2} \rho / \mu$, the Reynolds number for squeeze film dampers

$t \quad$ Time

$V_{t} \quad$ Precession velocity of the journal

$w \quad$ Thickness of piston rings

$x(t) \quad$ Displacement in the time domain

$X_{0} \quad$ Constant term from Fourier transformation

$X_{c i} \quad$ Cosine term from Fourier transformation

$X_{s i} \quad$ Sine term from Fourier transformation

$z \quad$ The axial direction 


\section{List of Figures}

1.1 SFD without Spring . . . . . . . . . . . . . . . . . 2

1.2 SFD with Spring . . . . . . . . . . . . . . . . . . . . 2

1.3 Responses $\mathrm{W} /$ and $\mathrm{W} / \mathrm{O} \mathrm{SFD}[1] \ldots \ldots \ldots \ldots$

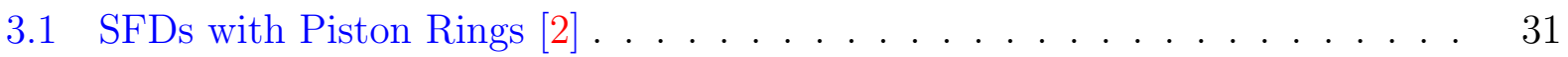

3.2 Fluid Film Region . . . . . . . . . . . . . . . . . . . . . . . . . 33

3.3 SFD Schematic View . . . . . . . . . . . . . . . . . . . 34

3.4 Pressure Distribution of a Damper with Piston Ring . . . . . . . . . . . . 36

3.5 Pressure Comparison in Axial Direction . . . . . . . . . . . . . . . 36

3.6 Pressure Comparison having Varying Radial Sealing Clearance of Piston Ring 37

3.7 Pressure Comparison with Different Leakage Coefficients . . . . . . . . . . 38

$3.8 \overline{C_{r t}}$ with Different $L / D \ldots \ldots \ldots \ldots$. . . . . . . . . . . . . . 40

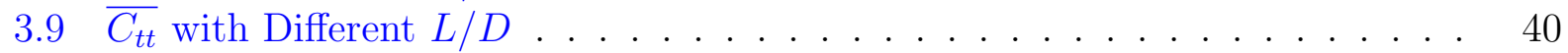

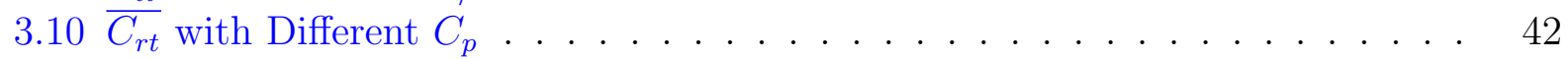

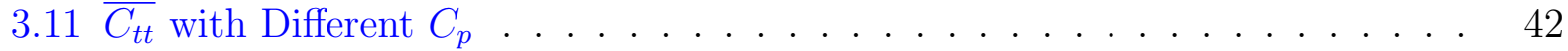

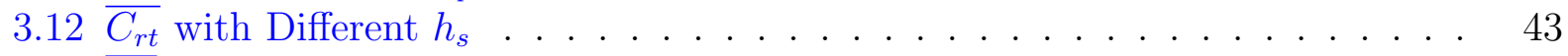

$3.13 \overline{C_{t t}}$ with Different $h_{s} \ldots \ldots \ldots \ldots \ldots \ldots \ldots \ldots$

3.14 Finite Difference Mesh for Hole Supply/Discharge . . . . . . . . . . . . . 45

3.15 Pressure Distribution with Piston Rings Seals and Hole Supply/Discharge . . 47

3.16 Pressure Distribution for Long Damper . . . . . . . . . . . . . . . . 48

$3.17 \overline{C_{r t}}$ of Cavitated Film with Varying Supply/Discharge Pressure . . . . . . . . 49

$3.18 \overline{C_{t t}}$ of Cavitated Film with Varying Supply/Discharge Pressure . . . . . . . . 50

$3.19 \overline{C_{t t}}$ of Cavitated Film (Fixed Eccentricity) . . . . . . . . . . . . 50

$3.20 \overline{C_{r t}}$ of Cavitated Film with Hole Number . . . . . . . . . . . . . 51

$3.21 \overline{C_{t t}}$ of Cavitated Film with Hole Number . . . . . . . . . . . . . 52

$3.22 \overline{C_{r t}}$ of Cavitated Film (Fixed Eccentricity) . . . . . . . . . . . . 53

$3.23 \overline{C_{t t}}$ of Cavitated Film (Fixed Eccentricity) . . . . . . . . . . . 53

3.24 Pressure Distribution with Groove Depth of $16 C \ldots \ldots$. . . . . . . . 55

3.25 Axial Pressure with Varying Groove Depth . . . . . . . . . . . . . . . 56

3.26 Pressure Distribution with Groove Depth of $16 C$ (With Holes) . . . . . . . 57

$3.27 \overline{C_{r t}}$ with Varying Groove Depth(Without Holes) . . . . . . . . . . . . . 59

$3.28 \overline{C_{t t}}$ with Varying Groove Depth(Without Holes) . . . . . . . . . . . . . . 60

$3.29 \overline{C_{r t}}$ with Varying Groove Depth(With Holes) . . . . . . . . . . . . . . . . . 61

$3.30 \overline{C_{t t}}$ with Varying Groove Depth(With Holes) . . . . . . . . . . . . . . . 61

$3.31 \overline{C_{r t}}$ with Varying Supply Pressure through Holes(Groove) . . . . . . . . . . 62 
$3.32 \overline{C_{t t}}$ with Varying Supply Pressure through Holes(Groove) . . . . . . . . . . . 64

4.1 Full Leakage $[1] \ldots \ldots$. . . . . . . . . . . . . . . . . . . . . . . . . . . . . 71

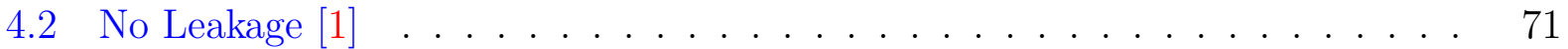

4.3 Typical Iteration Scheme . . . . . . . . . . . . . . . . . . . . . . . . . . . . . . . . 73

4.4 Illustration of Curve Intersection Method . . . . . . . . . . . . . . . . . . . . 74

4.5 Spring-Damping-Mass Model of SFD with Journal . . . . . . . . . . . . . 76

4.6 Finite Element Model of a Three Mass Rotor with SFD . . . . . . . . . . . . 77

4.7 Scheme of Unbalance Responses Analysis with CIM . . . . . . . . . . . . . 80

4.8 Comparison of Responses W/ and W/O Damper . . . . . . . . . . . 81

4.9 Responses of Damper Subject to Varying Unbalance Locations . . . . . . . . 82

4.10 Responses of Damper Subject to Varying Unbalance on Disk 2 . . . . . . . . 83

4.11 Responses of Left Bearing Subject to Varying Unbalance on Disk 2 . . . . . 83

4.12 Responses of Damper Subject to Varying Clearance Ratio . . . . . . . . . . 85

4.13 Responses of Right Ball Bearing with Varying Clearance Ratio . . . . . . . . 85

4.14 Amplification Factor V.S. Clearance Ratio . . . . . . . . . . . . . . . . 86

4.15 Responses of Damper with Varying Aspect Ratio . . . . . . . . . . . . . . 87

4.16 Responses of Right Ball Bearing with Varying Aspect Ratio . . . . . . . . . 88

4.17 Mode Shape Comparison at $8000 \mathrm{rpm}$. . . . . . . . . . . . . . . . 91

4.18 Log Decrement V.S. Cross-Coupled Stiffness . . . . . . . . . . . . . . . . . . 91

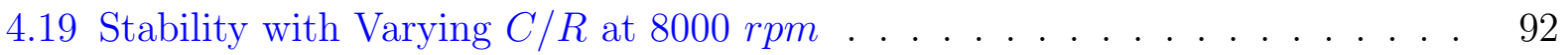

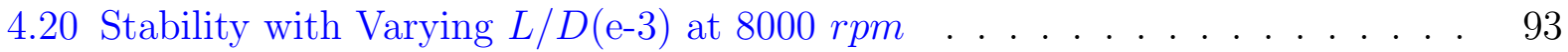

5.1 Iteration of a corrector $[3] \ldots \ldots \ldots \ldots$. . . . . . . . . . . . . 107

5.2 The Scheme of Improved Harmonic Balance Method . . . . . . . . . . . . . . 108

5.3 Response of Damper, $\mathrm{U}=0.6$ oz-in . . . . . . . . . . . . . . . . . . 109

5.4 Response of Damper, $\mathrm{U}=0.7$ oz-in on Disk 1 . . . . . . . . . . . . . . . 110

5.5 Response of Damper, $\mathrm{U}=0.7$ oz-in on Disk $2 \ldots \ldots$. . . . . . . . . . . . . 110

5.6 Response of Damper, $\mathrm{U}=0.7$ oz-in on Disk $3 \ldots \ldots$. . . . . . . . . . . . 111

5.7 FFT of Response at $3990 \mathrm{rpm}$, with Unbalance on Disk 2 . . . . . . . . . . . 111

5.8 Response of Damper with Load, $\mathrm{U}=0.6$ oz-in on Disk 2 . . . . . . . . . . . . 112

5.9 Response of Damper, with Varying Loads on Disk 3 . . . . . . . . . . . . . 113

5.10 Orbits at $7000 \mathrm{rpm}$, with Varying Loads on Disk 3 . . . . . . . . . . . . . . . 114

5.11 FFT of orbits $(7000 \mathrm{rpm})$ with Varying Load . . . . . . . . . . . . . . . 115

5.12 Response without Gravity . . . . . . . . . . . . . . . . 116

5.13 Response with Gravity . . . . . . . . . . . . . . . . . . . . . . . 117

5.14 Orbit at $2000 \mathrm{rpm}, \mathrm{K}=1500 \mathrm{lbf} / \mathrm{in} \ldots \ldots \ldots \ldots$

5.15 FFT of Response at $2000 \mathrm{rpm}, \mathrm{K}=1500 \mathrm{lbf} / \mathrm{in} \ldots \ldots$. . . . . . . . . . . 119

5.16 Response with Varying Inertia of Disk 1 . . . . . . . . . . . . . . . . 120

5.17 Response with Varying Inertia of Disk $2 \ldots . . . . . .120$

5.18 Response with Varying Inertia of Disk 3 . . . . . . . . . . . . . . . . . . 121

5.19 Comparison of Responses between HBM and Force Coefficients . . . . . . . . 122

5.20 Elliptical Orbit at $7490 \mathrm{rpm}$ using HBM . . . . . . . . . . . . . . . . . . 123

6.1 Cut-away of Rolls-Royce AE $3007[4] \ldots \ldots$. . . . . . . . . . . . . . 126 
6.2 Schematic View and FEA Model of LP Rotor . . . . . . . . . . . . . . . . 127

6.3 Orbits of Fan-End Damper Comparison . . . . . . . . . . . . . . . . . . . . 128

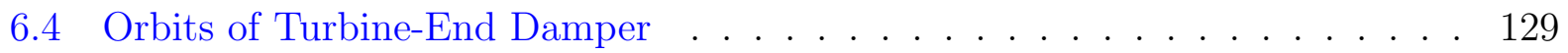

6.5 FFT of the response at $3500 \mathrm{rpm} \ldots \ldots \ldots$. . . . . . . . . . . 130

6.6 Responses of Fan-End Damper, UT=2 oz-in . . . . . . . . . . . . . . . . 132

6.7 Responses of Turbine-End Damper, UT=2 oz-in . . . . . . . . . . . . . 132

6.8 Responses of Fan-End Damper, UF=2 oz-in . . . . . . . . . . . . . . 133

6.9 Responses of Turbine-End Damper, UF=2 oz-in . . . . . . . . . . . . . 134

6.10 Response of Fan-End Damper with varying KF . . . . . . . . . . . . . . . . 135

6.11 Response of Turbine-End Damper with varying KF . . . . . . . . . . . . 135

6.12 Response of Fan-End Damper with varying KT . . . . . . . . . . . . . . 136

6.13 Response of Turbine-End Damper with varying KT . . . . . . . . . . . . 137

6.14 Schematic View and FEA Model of HP Rotor . . . . . . . . . . . . . . . . 138

6.15 Damper Responses with Varying L/D . . . . . . . . . . . . . . . . . . . 140

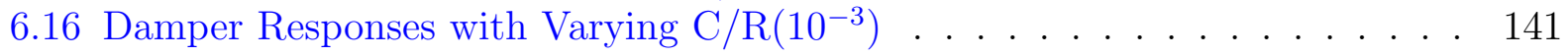

6.17 Damper Responses with Varying Spring Stiffness . . . . . . . . . . . . . . . 142

6.18 HP Rotor Natural Frequency with Varying Aspect Ratio . . . . . . . . . . . 144

6.19 HP Rotor Log Decrement with Varying Aspect Ratio . . . . . . . . . . . . . 144

6.20 Stability Margin with Varying Aspect Ratio . . . . . . . . . . . . . . . . . . 145

6.21 Stability Margin with Varying Clearance Ratio . . . . . . . . . . . . . . 146

6.22 Natural Frequency with Varying Clearance Ratio . . . . . . . . . . . . . . . 147

$6.23 \mathrm{Log}$ Decrement with Varying Clearance Ratio . . . . . . . . . . . . . . . . 147

6.24 Natural Frequency with Varying Stiffness . . . . . . . . . . . . . . . . . . . 148

6.25 Log Decrement with Varying Stiffness . . . . . . . . . . . . . . . . . . 148

6.26 Natural Frequency with Varying Stiffness (Wider Range) . . . . . . . . . . . 149

6.27 Log Decrement with Varying Stiffness (Wider Range) . . . . . . . . . . . . . 150

6.28 Journal Orbits with Supply Pressure(15,200rpm) . . . . . . . . . . . . . 152

6.29 Orbits of Right Bearing with Supply Pressure(15,200rpm) . . . . . . . . . 153

6.30 Journal Orbits with Groove Depth(15,200rpm) . . . . . . . . . . . . . 155

6.31 Orbits of Right Bearing with Groove Depth(15,200rpm) . . . . . . . . . . . . 156

6.32 Damper Orbits with Supply Pressure (Hole Supply) . . . . . . . . . . . . . 157

6.33 Orbits Right Bearing with Supply Pressure (Hole Supply) . . . . . . . . . . . 157 


\section{List of Tables}

3.1 Circumferential Location of Holes . . . . . . . . . . . . . . . . . . . 51

4.1 Damping Coefficients of Open-end SFDs under CCO . . . . . . . . . 70

4.2 Force Coefficients of Open-end SFDs W/ inertia [5] . . . . . . . . . . . . 72

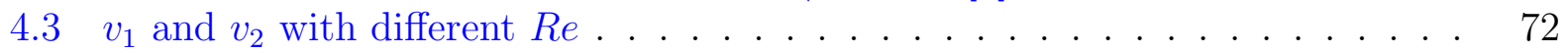

4.4 Geometries of the Rotor (unit: inch) _ . . . . . . . . . . . . . 79

4.5 Geometries and Operating Conditions of the Damper . . . . . . . . . . 81

4.6 Frequency and Log Dec Comparison, W/ and W/O SFD . . . . . . . . 90

5.1 Geometries and Operating Conditions of the Damper for HBM . . . . . . 108

6.1 Comparison of Computational Time . . . . . . . . . . . . . . . . . 129 


\section{Chapter 1}

\section{Problem Statement}

\subsection{Introduction}

In the 50 years since its invention, Squeeze Film Dampers(SFDs) have seen widespread use in rotor-bearing systems, including turbines and compressors. The SFD has become one of the crucial components in these machines for reliable operation with demanding requirements. The main factor for this popularity is its ability to produce additional damping to attenuate vibrations and improve stability of rotating systems that operate above the first few critical speeds to achieve constant needs of high efficiency and high power-density. Much attention

has been paid to understand SFD's performance, and problems remain to be solved to improve the understanding of this complicated component in rotating systems.

The mechanism of damping generation inside dampers is the squeezing motion of a viscous lubricant film between the inner ring and outer ring. As shown in Figure 1.1, the inner ring includes the rotating shaft $\mathbf{S}$, a ball bearing $\mathbf{B}$ and the journal $\mathbf{J}$. The outer ring contains the housing $\mathbf{H}$. There is a anti-rotation pin $\mathbf{P}$ between the journal and the housing to prevent nonsynchronous rotation of the inner ring. Thus, the inner ring is precessing inside the viscous lubricant film $\mathbf{F}$, and some of the vibration from the shaft is absorbed.

Figure 1.1 shows a damper without a squirrel cage (or centering spring), and the inner 


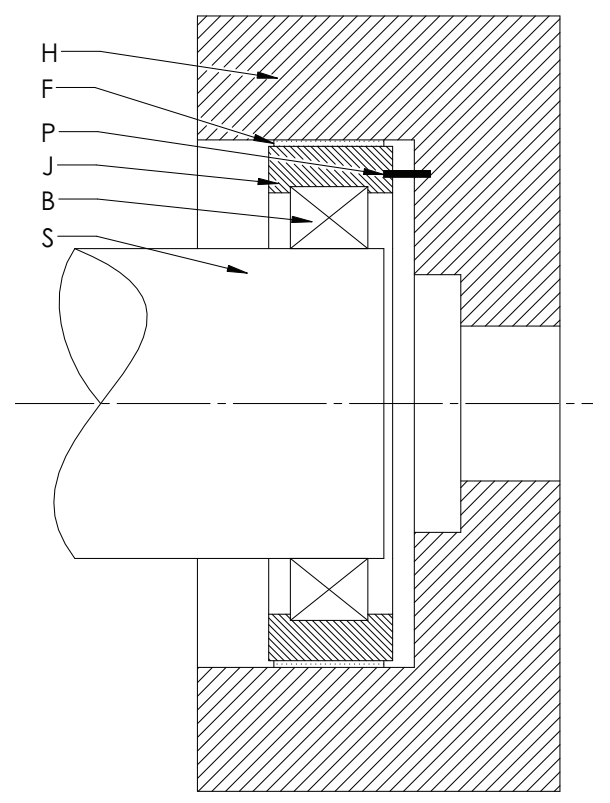

Figure 1.1: SFD without Spring

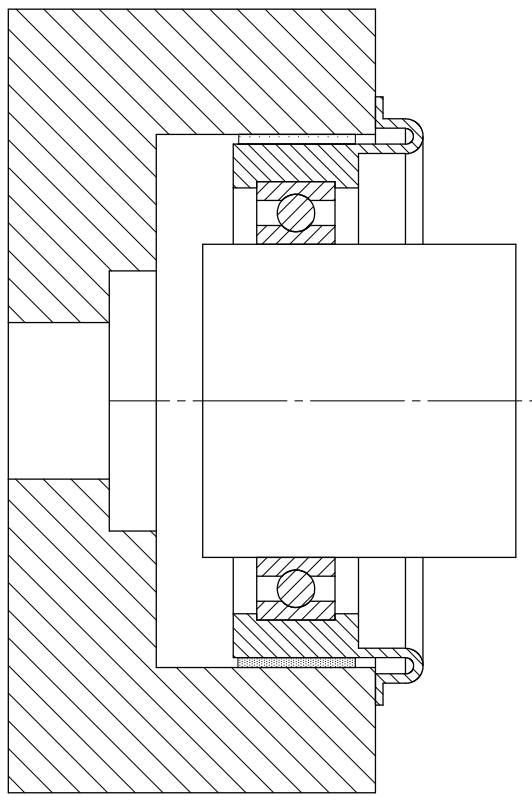

Figure 1.2: SFD with Spring

ring touches the bottom of the outer ring when the shaft is not rotating. Therefore, this kind of assembly is usually employed in systems with light external loads. For large systems, a centering spring is used, as depicted in Figure 1.2. The centering spring and journal are a single piece in the given figure. The U-shaped cage, which is widely used in aero-engines, connects the inner ring to the housing and saves axial space at the same time. For both configurations, the squeezing film plays the critical role in dissipating vibrations from the rotating shaft. This is the key function that squeeze film damper is used to improve the vibrational performance of rotating machines.

Figure 1.3 shows an example of a centrifugal compressor before and after installation of squeeze film dampers. The compressor was encountering a costly instability problem due to self-excited whirl before reaching the design operating speed at 13,500 rpm. After retrofitting the previous bearing design with a squeeze film damper, the compressor was able to operate at the design speed successfully without any sub-synchronous vibration frequency component. At the same time, the compressor's performance was greatly improved with an increase in maximum discharge pressure from 175 psi to 650 psi [1]. 


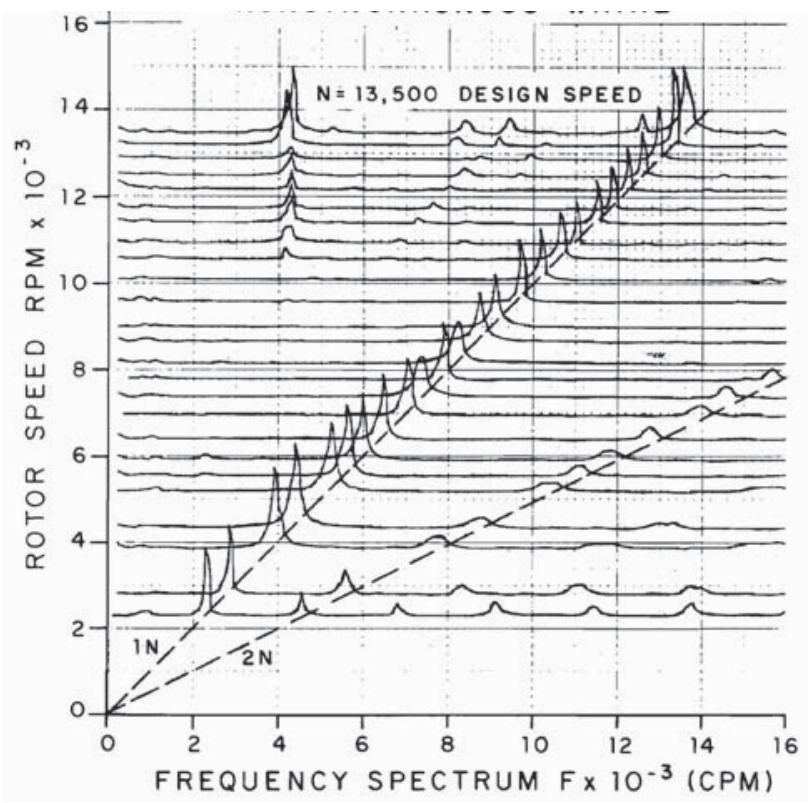

(a) Without SFD

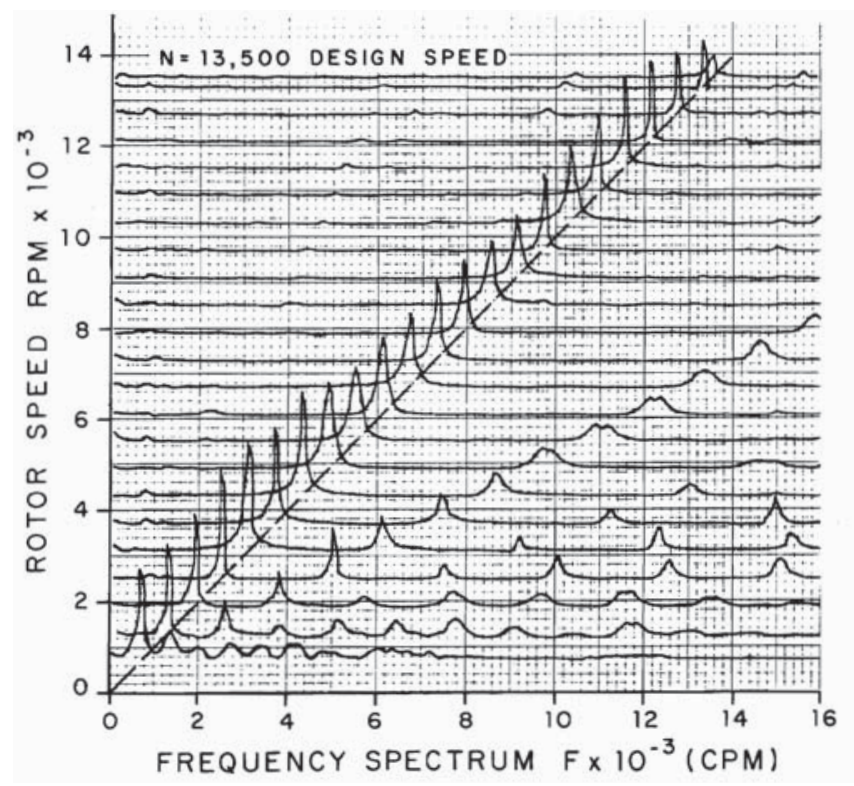

(b) With SFD

Figure 1.3: Responses W/ and W/O SFD [1]

However, not every application of squeeze film damper would give desirable results as the example shown above. Improper damper design might provide too much or too little damping and result in even worse performance than that without installation of SFDs. The main reason behind this is that there are multiple factors affecting a damper's damping 
capacity, including damper geometry (especially the film land), lubricant properties, sealing conditions of axial ends and lubricant supply and discharge conditions. Meanwhile, each factor contains its sub-factors. Any difference in these factors produces different hydrodynamic forces in the film and therefore results in varying rotordynamic behavior of the whole system. Therefore, to avoid inappropriate design and to take advantage of improved stability using SFDs, numerous studies have been focused on the effects of each factor and have tried to maximize the advantages. However, one major situation remains unsolved, and it is the failure of current modeling tools that can accurately predict the performance of a realistic squeeze film damper at both the component and system level.

\subsection{Problems}

For decades, modeling of SFD has been extensively based on the simplest geometries in a laboratory setting. The pressure distribution around the journal is obtained with many simplifying assumptions, such as short/long bearing approximations, isoviscous lubricants, open-end sealing condition and constant hydrostatic pressure within central grooves. However, these presumptions do not apply to most real rotating machines such as aero-engines. When it comes to system level analysis for the evaluating effects of squeeze film dampers on rotordynamics, rigid rotor or simple flexible rotor with few Degrees Of Freedom(DOF) are used extensively. These two aspects, from both component and system levels, indicate the need for improvements in SFD modeling and simulation.

\subsubsection{Component Level}

The component level analysis of SFDs mainly focuses on the hydrodynamic forces from the lubricant film. The hydrodynamics rely on damper geometry, lubricant, sealing, supply and discharge conditions. Many simplifications have been made to these operating conditions in 
prior studies. These fail to give convincing analytical results when compared to practical experience.

\section{Damper Geometry}

The main assumption in damper geometry is related to damper axial length. Either the short bearing or long bearing assumptions are employed extensively to produce simple, closed form solutions. The former assumption holds when the aspect ratio L/D is smaller than 0.25 , then the pressure gradient in the circumferential direction is considered to be much smaller than that of the axial direction and can be neglected. The latter assumption neglects the pressure gradient in the axial direction when the aspect ratio is much larger than 1 , and it is referred to as an infinite length damper. Each presumption will give an explicit form of pressure distribution around the journal. The corresponding force coefficients of the damper would then be possible to be written as closed-form expression.

Although these two assumptions shed light on understanding the basic behaviors of the damper, they can not obtain accurate results for finite length dampers which have aspect ratio between the above two cases. No closed form solution of the pressure is currently known when using the classic Reynolds equation. Some studies combine the solutions of both short and long damper by introducing a weighting factor and forming an explicit expression for the damper forces. The accuracy of this hybrid equation depends on the weighting factor, which is unknown for a given damper. To achieve better predictions, experiments are required to calibrate the factor before performing further analysis. Furthermore, this combined form is not useful during the design stage of a damper, since the weighting factor cannot be determined in advance.

\section{Lubricant Properties}

Lubricant properties include two elements that relate directly to the capacity of damping generation, they are the lubricant density and viscosity. In the simplest analytical model of a 
damper, these two are assumed to be uniform through the entire lubricant film. Lubricant film is considered to be incompressible and isoviscous in classic lubrication theory. However, such simplifications do not represent the application of SFDs in industry and result in inaccurate predictions when compared to experimental measurements. One crucial phenomenon for dampers in real-world applications is cavitation, which is unavoidable in most machines supported on SFDs. There are two main forms of cavitation; gaseous and vaporous cavitations. Both forms result in compressible lubricant, which can not be accurately modeled with classical lubrication theory. Since both kinds of cavitation not only reduce the density with air-oil mixture but also increase the effective viscosity with surface tension produced by the boundary between gas and liquid in the lubricant film.

In typical theoretical analyses, the cavitation is considered by imposing the $\pi$-film pressure boundary condition, which truncates the negative pressure region with ambient pressure while keeping the positive pressure zone. As the name suggests, $\pi$-film assumes that half of the damper circumference is under ambient pressure. Although this pressure form is widely used, it is only valid for the approximation of the gaseous cavitation but not the vaporous. It also fails to consider the influences on both lubricant density and viscosity. Furthermore, experiments [6] show that the gaseous cavitation can not only happen under negative pressure but also exists in the positive zone with rotating pressure wave. These two phenomena can not be handled by the $\pi$-film approximation.

On the other hand, the uncavitated squeeze film is extensively modeled with a $2 \pi$-film form in theoretical analysis, which retains the original negative pressure without any truncation. However, this form does not give reasonable results when compared to experimental observations[6]. The $2 \pi$-film approximation is found to be only suitable for low speeds, which generates negative pressure with low magnitude. As the rotational speed increases to the designed operating region, the pressure continues to decrease in the negative regime until the vapor cavitation occurs. This prevents lower negative pressure due to the vapor pressure of the lubricant. With even higher speeds, the vaporous cavitation occupies a high percentage 
of the negative regime and generates a large enough pressure difference between the pressure inside the film and outside the end seal to draw in air from the surroundings, resulting in mixed cavitation with both gaseous and vaporous form. Neither $\pi$-film nor $2 \pi$-film can model this mixture, and no analytical model exists currently.

Another fact that is neglected in typical analyses is the temperature variation inside the squeeze film. The temperature is assumed to be constant, although the squeeze film works by dissipating the vibrational energy from the journal, which is then converted to thermal energy. The main argument about the validity of the assumption is that the lubricant is supplied and discharged with a high flow rate which results in constant, steady-state temperature. This might be true if the damper is open-ended and under flooded operating conditions. However, for dampers in industrial applications, which usually have seals such as piston rings and O-rings at the axial ends to improve damping capacity and discharge the oil through grooves or orifices, this assumption is not accurate. These configurations can reduce the flow circulation rate and inevitably result in an increase of the film temperature, producing changes in lubricant properties, especially the viscosity. Therefore, a thermodynamic analysis coupled with the hydrodynamic analysis is needed for accurate predictions.

\section{Sealing Condition}

Typical sealing conditions for SFDs include open-end, O-ring seals, piston rings and side plates. Although the open-end condition has popularity in the academic community due to its simplicity, it is not often seen in industrial applications. The main reason is that the open-end condition has much less damping capacity compared with other sealing methods and is unable to satisfy practical requirements. Therefore, O-ring, piston rings and side plates are preferred for most applications in the real world. One of the challenges to model these realistic sealing conditions is the way to consider corresponding leakage through these seals. Since the complete end sealing is prone to increase lubricant temperature and reduce the damping, controlled leakage is favored. Thus, reliable analysis must consider the effect of leakage on the pressure distribution of the damper. 
A classic method of including the effect is to introduce a leakage factor to relate the pressure and pressure gradient at the sealing end. However, the value of the factor, which is the key to precise prediction, is assumed based on experience. It depends not only on the end-seal geometry but also on the dynamics of the journal. Thus, some kind of benchmark is needed for accurate predictions for a given system. A consequence is that the factor introduced for a specific configuration can not be generalized.

Besides their effects on the pressure distribution of the squeezing film, end seals have inherent elastomeric characteristics giving additional damping and stiffness to the damper[7][8]. Although these inherent coefficients are much less than those generated by the squeeze film for large and heavy rotor-bearing systems, they might be comparable to oil-film effects for small and light systems within which the built-in properties of seals cannot be neglected, resulting in a more complicated model for precise analysis.

\section{Supply and Discharge Condition}

Similar to the end-sealing conditions, the supply and discharge conditions determine the boundary conditions when calculating the damping capacity of a damper. Different supply and discharge configurations produce various boundaries and consequently give diverse pressure distributions. Common supply methods include axial-end inlet, centered groove, off-centered groove and orifice hole, which can also be the forms for discharging. The axial-end form is widely accepted in theoretical analysis and laboratory testing due to its simplicity. However, it is not that common in industrial rotating machines such as aero-engines because of its disadvantages in damping generation. The form of groove and hole, on the other hand, is preferred to increase damping performance, based on operational experience.

The classic hypothesis for groove supply/discharge is that the pressure inside the groove is constant and equal to supply/discharge pressure. However, experiments [9][10] demonstrate a quite different story. The groove generates totally different values from the supply/discharge pressure, even when the effects of fluid inertia and turbulence inside are accounted for. Depending on the groove geometry, groove depth and axial length, the inside pressure can 
have the same order of magnitude as the generated by the nearby film land. Therefore, this traditional hypothesis in analysis will not predict the real world.

For cases requiring high damping capacity, an orifice hole is a better choice than the groove [11]. One of the common methods in dealing with this boundary is to equate the pressure at the orifice to the supply/discharge condition. The rotating pressure wave with journal precession invalidates this assumption with inversion of lubricant flow in the holes [12]. To give precise predictions, the consideration of back flow is required.

\subsubsection{System Level}

Besides the inappropriate hypotheses for the modeling of squeeze film damper at the component level, the incorporation of dampers at the system level fails to accurately predict the dynamics of rotor-bearing systems for most industrial applications. The questionable situation for the systems level can be summarized as an oversimplified model treating the system as plain rotor-bearing systems supported on an ideal squeeze film damper. The characteristics of systems commonly analyzed include, but are not limit to, rigid rotors with axial symmetry, simple flexible rotors with few Degrees of Freedom (DOFs) and constrained operations such as circular centered orbits or off-centered orbits with small amplitude.

Typical damper models for system level validation include the short/long bearing assumption, open-end sealing conditions and $\pi$-film/2 $/$-film assumption for cavitation. However, as described in Section 1.2.1, these models fail to predict pressures inside realistic dampers accurately and therefore give unreasonable rotordynamic behaviors at the system level. Furthermore, those typical analyses neglect the fluid inertia, which was found to be critical for SFDs from both theoretical analysis [13][14] and experiments [15]. The inertia effect is observed not only to the changing of the critical speed of the system but also to reducing the non-linear jump phenomenon in unbalance responses.

Another widely applied hypothesis in rotordynamic analysis of SFD-supported systems is circular orbits for the precessing journal. The orbits might be centered [16] [17] or off-centered 
[18] with small amplitude. The main purpose of the hypothesis is to obtain expressions in closed-form for the damper coefficients as functions of damper geometries and precession speed. These coefficients are then incorporated into the rotor-bearing system analysis and determined through iteration. The problem is that the corresponding results are valid only if the shape of orbits are circular and have small amplitude. They cannot be generalized to dampers supporting complex rotating systems such as aero-engines, which are able to produce non-circular orbits.

The fact under the constrained-orbits assumption is that no explicit form of damper coefficients is known for more complicated orbit shapes. For dampers with end sealing using piston rings or O-rings and supply/discharge forms of groove or orifice, even if the journal precesses circularly, these coefficients are not accurate. Therefore, the method to model SFDs with closed-form dynamic coefficients should not be adopted for accurate prediction of practical industrial systems. Instead, numerical methods must be employed for the analysis of realistic dampers at the system level.

For nonlinear numerical methods, the influence of SFDs in the whole rotor-bearing system is represented by external hydrodynamic forces, which comes from the integration of the pressure distribution around the film land. Then, the consideration of complex damper configuration becomes possible for system evaluation. There are two categories based on the domain within which damper forces are expressed, the time domain or the frequency domain. A typical method in time domain analysis is the Runge-Kutta method. Examples in $[19,20,21]$ use this method to consider the effects of SFDs at the system level. As shown by those examples, the rotating systems considered are either rigid or flexible rotors with simple and symmetric structures, which are not able to represent complicated machines supported on SFDs. Another concern when using Runge-Kutta method is its computational inefficiency. It takes most of the computation time for the transient response before obtaining steady state responses, no matter the complexity of the rotating system. In addition, the steady state results from this method depend on the initial guess of the analysis if a rotating system 
has multiple solutions, which is possible due to the nonlinearity of SFDs[22].

Analytic methods in the frequency domain, such as the harmonic balance method(HBM) [23, 24, 25] and trigonometric collocation method(TCM) [26][27], are able to obtain the responses for multiple solutions given different initial guesses. Additionally, they are much more efficient to obtain steady state responses than those in the time domain. One key hypothesis for the HBM or TCM method is periodic or quasi-periodic responses of steady state. Therefore, the methods in the frequency domain cannot replace time domain analysis if the rotating system acts non-periodically or chaotically. Similar to those systems employed in the time domain, the academic community has extensively applied the frequency domain method to simple rotor-bearing systems on ideal SFDs, neglecting the effects such as gyroscopics, external loads and flexible foundations, which are critical to predicting the response of practical rotating systems in industry.

\subsection{Dissertation Outline}

As described in the previous section, the classic SFD analytical models rely on a number of simplifying assumptions that neglect important physical phenomena. The improved SFD model developed in this dissertation can be readily incorporated into larger, systemlevel, rotordynamic models to provide more accurate evaluation of SFD performance at the design stage of new machines or in the analysis of existing equipment. For the component level analysis, the main shortcomings exist in the models for dampers with end seals and supply/discharge conditions. At the system level, the modeling of SFDs using closed-form solutions as done in the previous works fails to predict the performance of rotating systems having large loads and can not ensure an effective optimized-design. When using the direct force method for the system level analysis, most of the published work considered the rotating system as either a rigid rotor or a simple flexible rotor with few degree-of-freedom. There are

several cases that looked at more complicated systems such as an aero-engine, but all the 
SFDs were modeled using open-end configurations, which are rare in rotating systems for practical industry applications including aero-engines. Therefore, to improve the existing studies, the aims of this work are: 1) to enable the analysis of realistic SFD configurations at the component level; 2) to improve the effectiveness of calculations of rotating systems with SFDs using a closed-form solution for initial parameter and system design studies; and 3) to realize the efficient and accurate system level analysis of complex practical industrial applications such as aero-engines. To accomplish these goals, the outline of this work is described as follows.

Chapter 2 gives the state of the art literature review on the status of both component and system level analyses, including lubricant properties, end-seal conditions, supply and discharge conditions, and forced responses of rotating systems with SFDs and their effects on stability. The review affirms that the objectives of the dissertation satisfy the needs of industrial applications with SFDs.

The component level analysis of SFDs is presented in Chapter 3. Three realistic configurations of industrial SFDs will be considered, including one end seal condition with piston rings and two supply/discharge conditions - hole and central groove. A numerical method, the finite difference method, is used to obtain the pressure distribution and damping capacity for these configurations. Both the cross-coupled and direct damping of these three cases are compared with simplified models such as the open-end model. The study of parametric changes on each configuration are evaluated as well. The results from this chapter will be applied to the system level analysis.

Chapter 4 focuses on both unbalance response and stability analysis of rotating systems with SFDs using closed-form solutions. Although the closed-form is a simplified SFD model, it provides fast solution time and gives accurate results if the required assumptions are satisfied. In addition, the closed-form solution can give reasonable approximations for complex industrial application which enables quick design and parametric studies. Thus, this simplified form can be useful in specific situations and, in particular, at the initial component and system design 
stage. To improve the use of closed-form solution models, a more effective procedure based on the Curve Intersection Method is developed to obtain the unbalance response, which is more robust than the traditional procedure and enables efficient parametric study on damper geometries. The method is evaluated through the analysis of a multi-mass flexible rotor. The optimized design of the supporting damper is discussed by evaluating the stability behavior of the system.

Chapter 5 focuses on forced response and stability analysis using the direct force method, which does not have the limitations associated with the closed-form solution. To enable the steady state analysis of rotating systems with realistic operating conditions, this chapter improves the direct force method in the frequency domain by using the idea of homotopy and the procedure of predictor-corrector. In addition, this work will consider important factors such as gyroscopic effects, which are neglected in most of the previous work, Chapter 5 models the rotor-bearing systems with SFDs by combining the finite element method and harmonic balance method. The developed method is applied to the multi-mass rotor with realistic operating conditions. Observation of the jump phenomenon and the importance of gyroscopic effects are documented.

To demonstrate the rotordynamics analysis of a practical rotor-bearing system with SFDs using the methods developed in both Chapter 4 and Chapter 5, the study of an aeroengine is considered in Chapter 6. The forced response of both Low Pressure(LP) and High Pressure(HP) rotors are discussed. The developed methods is shown to be at least five times more efficient than the traditional Runge-Kutta method. To understand the behavior of the SFD within the LP rotor, the study of different operating conditions is included. For the HP rotor, the initial damper design with the maximum stability margin is found by optimization using the closed-form solution approach. Based on the geometries from this initial design study, SFDs with realistic configurations are then evaluated using the models developed in Chapter 3. Two popular damper configurations in aero-engines, piston ring seal with central groove supply/discharge and piston ring seal with direct hole supply/discharge, are included 
for the HP rotor analysis. This chapter illustrates the shortcomings of the simplified damper model for SFDs in a realistic configuration and demonstrates the effectiveness of the methods developed in this work.

The conclusions of this work are in Chapter 7, which summarizes the main contributions and presents possibilities for further investigation. 


\section{Chapter 2}

\section{Rotor-Bearing Systems with SFDs:}

\section{Review}

The state of art review of both the component and system level analyses are included in this chapter. The hydrodynamic aspect of SFD is described first, followed by the consideration of SFD in rotor-bearing systems at the system level.

\subsection{Lubricant Properties}

\subsubsection{Cavitation}

Cavitation in the oil film is due mainly to two reasons: the low supply pressure of lubricant and imperfect end-sealing in reality. It can not be modeled accurately with classical lubrication theory [28]. The two typical forms of cavitation, lubrication vaporization and gaseous entrance, lead to a two-phase mixture in the squeeze film lubricant. White [29], extending the experiment by Cooper[30], observed that bubbles inside lubricant persisted in the high pressure region when the eccentricity ratio is larger than 0.3. Hibner and Bansal [31] experimentally found that the pressure profile and hydrodynamic forces did not correspond well to the results from classical lubrication theory, when the squeeze film was under the influence of cavitation. 
Zeidan and Vance [32], with the help of high-speed photography, observed the occurrence of both vapor and gas cavitation. The vapor cavitation was found with a few bubbles at the beginning and collapsed as pressure increased. However, the bubbles of gas cavitation shrank in size and persisted throughout the high pressure region. They also observed that gas cavitation reduced the damper load capacity dramatically, and the decreased bubble size in the high pressure region led to a homogeneous mixture of air and oil. This observation indicates that the assumption of an incompressible fluid is not valid in SFDs.

Zeidan and Vance [6] defined five distinct cavitation regimes subject to different operating speeds, examined their characteristics of pressure distribution and studied the influences of supply pressure on the distribution. Vapor cavitation was observed to have effects on the negative pressure region only. A pressure overshoot occurred near the location where pressure transfers from negative to positive, as a result of implosion of cavitation bubbles taking place. The gaseous cavitation was also observed to not only affect the pressure wave dramatically in the negative pressure regime, but also narrow the span of the positive pressure regime.

Diaz and San Andrés [33] examined the effects of air/oil bubbly mixture on pressure in a squeeze film damper executing a circular centered orbit with constant whirling speed. The increased amount of air was reported to decrease the peak-to-peak dynamic pressure and viscosity of the lubricant and increase journal orbit radius. A region with constant pressure was also observed when a high volume value of air to oil ratio was present. They described the gaseous cavitation for bubbly mixture as an unstable phenomenon in the sense that the film dynamic pressure was not repeatable with successive journal motion.

Diaz [34] extended the previous work with two different whirling frequencies and adopted a period averaging method to measure the variation of pressure distribution under the effects of air/oil mixture. They found that the radial force component of the damper was relatively insensitive to the change of an air/oil ratio under 0.85 , the tangential component decreased linearly as the ratio increased, and both force components became zero for a pure air condition.

With the same test rig as in [34], Diaz and San Andrés [35] investigated the fundamental 
difference between vapor and gas cavitation under the influence of whirl speed and supply pressure. They found that the region of vapor cavitation increased linearly with precessing speed, while the gas cavity did not vary much. For the peak-to-peak pressure, vapor cavitation remained nearly constant with precessing speed compared to the variance for the case of gas cavitation. The increase of supply pressure was also observed to reduce the air entrainment effect and increase the zone of vapor cavitation. Both tangential and radial forces were insensitive to whirling speed for air entrainment, but increased linearly for vapor cavitation. The prediction from the short bearing model with open ends was reported to be comparable with experimental results having the vapor cavitation effect. This indicates that the assumption of an open-end short bearing is invalid for gas cavitation.

Although several experiments have been carried out to understand the characteristics of cavitation and bubbly mixture inside SFDs, little analytical work is available to model them accurately. One reason for this situation is that the results from classical lubrication theory do not result in a good correlation with experimental data[28]. Tao et al. [36], under the assumption of isothermal flow without effects of cavitation, developed an analytical model for bubbly lubricant. A Reynolds-like lubricant equation, within which the effective viscosity of the lubricant was modeled as a quartic function of the volume fraction of air, was used to predict the mixture pressure. Favorable agreement with experimental data was achieved. Diaz and San Andrés [37] obtained a modified Reynolds equation for the prediction of squeeze film damper pressure in a homogeneous bubbly mixture, considering the mixture density and viscosity as functions of the effective gas volume fraction. An application of this model to an open-end damper gave good agreement with experimental results on the damper forces and peak-to-peak pressure.

\subsubsection{Fluid Inertia}

The influences of fluid inertia on squeeze film forces have been overlooked by classical lubrication theory, which produces discrepant results from experimental observations [15] 
[38]. SFDs for practical applications usually have squeeze film Reynolds number ( $R e=$ $\rho \omega c^{2} / \mu$ ) over a range of 1 to 50[39], and the influence of fluid inertia can be large enough to affect the dynamics of the journal. Many investigations have been done from both theoretical[14, 40, 41, 42] and experimental [15, 43, 44] aspects.

There are two main approximations that are widely used to consider the inertia effects in modeling, the momentum and the energy approximation. The former integrates the continuity equation in the Navier-Stokes $(\mathrm{N}-\mathrm{S})$ equation along the film thickness, and is also known as the average inertia method. The velocity profile for this method is assumed to be the same as that in the absence of inertia effects[45]. The momentum approximation can also be extended for the use of perturbation methods which express the pressure distribution and velocity profile in terms of $R e$.

San Andrés and Vance[14] and Tichy[46] verified that the results derived from small value of $R e$ can be used for higher value when $R e<25$. In [41], a finite-length damper with assumed Circular Centered Orbits(CCOs) was investigated using a perturbation method which expresses the pressure in terms of zero-order, first-order. The force coefficients were obtained, including both effects of $L / D$ (aspect ratio) and local end-sealing conditions.

The energy approximation, on the other hand, considers the kinetic energy of the fluid. However, the results from this method have about $20 \%$ difference between those from the momentum approximation for CCOs with small amplitudes[47]. Zhang et al.[48] claimed that the inertia coefficients from both approximations can have the same expressions with two different coefficients depending on which approximation was used.

San Andrés[49] experimentally observed the significance of fluid inertia on damper pressure and forces for the range of $R e$ from 2 to 6 . A large clearance of the damper is adopted for the purpose of increasing the inertia effect. The damper precessed along circular centered orbits with amplitude smaller than $50 \%$ of radial clearance, different from others such as [15] [50] having orbits with small amplitude. A prediction tool using the finite element method gave a favorable pressure comparison with measurements. 


\subsection{Sealing Condition}

The main purpose of end-seals is to reduce axial end leakage and increase the damping capacity of a damper, although end-seals can result in higher film temperature and lower viscosity of the lubricant. From the modeling point of view, the most challenging part is to relate the axial leakage with the pressure drop at the ends. O-ring and piston ring seals are the two most popular types in the real world.

\subsubsection{O-rings}

The O-ring seal has two different types based on its arrangement, radial O-rings and side O-rings. The radial O-ring deforms with a circumferential pressure distribution, which increases the flow rate in axial direction. The balance of flow along the circumferential direction, together with the introduction of a outflow coefficient, leads to an analytical model for this type of O-ring[2]. The side O-ring can be modeled without consideration of the circumferential pressure gradient in the seal, since the width of the O-ring is usually much larger than the radial clearance of the damper[2]. Since O-rings are typical elastomeric seals, their inherent stiffness and damping properties can affect the dynamics of the whole system [7][8]. However, these inherent characteristics have less importance than those related to end leakage effects with pressure drop[51], which underlines the significance of end seal boundaries.

\subsubsection{Piston Rings}

An analytical model of a piston ring can be obtained from the local boundary between the axial pressure drop through the seal and the pressure gradient inside the seal[2]. A leakage coefficient, a positive real number, is included in the model. However, the value of the coefficient is dependent on actual operating conditions. Empirical data or additional experience is needed for accurate predictions. In other words, a coefficient for one system can 
not be generalized. Unlike O-ring seals, the open literature shows little work has been done to understand the dynamic behaviors of piston rings inside SFDs. Jager et al.[52] evaluated the behaviors of piston rings, focusing on the demonstration of contact surfaces and the ring rotation phenomena. The leakage was found not only at the location of closed gap but also in the circumferential direction. They also observed the leakage effects on the damping forces through experiments and the decrease of seal effects as the amplitude of motion increases.

\subsection{Supply and Discharge Condition}

Different forms of groove and hole for both supply and discharge conditions are widely used in practical applications. The considerations of these two forms for analytical models are similar to those of sealing conditions since their contributions to the pressure distribution can be counted as boundary conditions as well.

\subsubsection{Groove}

In classical analytical models, the pressure inside the groove is usually prescribed as the supply/discharge pressure. Dampers with central grooves are considered to have two separated film lands, which do not interact with each other. However, the experiments contradict this theory, as the pressure within the groove can have the same order of amplitude as that generated by the film lands [7] [53]. The measurements also verify that complex flow interactions happen between the groove and squeeze film lands. In [10] and [54], the damper acts like a single film land when the journal precesses within small amplitude centered orbits, despite the presence of a central groove.

The ratio of groove depth to damper radial clearance plays another important role in the pressure distribution inside the groove. Arauz and San Andrés[10] found that the higher the ratio, the lower the pressure produced inside groove. When the ratio equals 3.5, the pressure in groove is over $60 \%$ of the value from a single film land. Levesley and Holmes[55] 
experimentally investigated the effects of the number of holes to the supply groove, the axial location of the groove and the end seal conditions on the unbalance responses. The reduction in the number of holes has little influence on the responses when the damper is sealed with piston rings, but produces detrimental results with end-chambered seals. The centered and off-centered groove supply show little difference for the weak seal condition. More recently, Delgado and San Andrés[56] verified the added mass effects due to the inertial forces inside the groove. The damping coefficients decrease with the increase of the groove depth as found in $[56]$.

\subsubsection{Orifice Hole}

The application of orifice holes for supply/discharge can improve the damping capacity of a damper, if the proper number of holes are chosen[11]. However, the pressure distribution with oil holes can be influenced by temporary reverse flow. Marmol and Vance[2] developed a boundary conditions for finite difference method to obtain the pressure distribution, based on the balance between the flow into the hole and the flow away from the hole. Chen and Hahn[11] solved for the pressure with a trigonometric power series, which frees the constraints on the location of hole and the eccentricity value. They also found that no significant interaction is possible between adjacent holes if they are at least 4 times the hole diameters apart, which is usually the case for most practical applications.

Walton II et al.[12] found that the arrangement of supply/discharge holes can have a significant influence on lubricant flow, the resulting pressure distribution and the rupture zone. Rodrigues et al.[57] used the finite difference method to study the influences of number of holes and the power supplied to pump on the responses and stability of a Jeffcott rotor. Five holes were found to generate a more stable system than two holes. They also verified that the higher the supply power the stiffer the damper, which increases the critical speed of the system. 


\section{$2.4 \quad$ Forced Response}

Two methods, the force coefficients and the direct force, are the main two in the forced response analyses for rotor bearing systems with SFDs. The basic idea of force coefficients is to model SFDs with explicit stiffness, damping and mass coefficients after giving the displacement(such as eccentricity), the velocity of the journal center and the acceleration. On the other hand, the direct force method consider the dampers' hydrodynamic forces directly, which are the functions of those dynamic properties assumed in the force coefficients method.

\subsubsection{Force Coefficients Method}

Since no closed form solutions are available for the pressure (or the hydrodynamic forces) from the generalized form of either the Navier-Stokes equation or the Reynolds equation, the force coefficients method requires assumptions to obtain the explicit dynamic coefficients. The assumptions are on the damper axial length, the motion of the journal and the end sealing conditions, including short or long bearing, centered or off-centered circular orbits and open-ends sealing. The cavitation are modeled with $\pi$-film and $2 \pi$-film for gas and vapor cavitation, respectively. Moreover, the supply and discharge conditions for damper models using this method are limited to a centered supply groove with constant pressure distribution inside the groove and axial end supply(or discharge).

The short or long bearing assumption[22, 58, 59, 60, 61, 62] is applied as appropriate based on damper aspect ratio due to the reduction of the two dimensional pressure profile to a one dimensional pressure profile. Therefore, the pressure can be expressed explicitly, and so can the hydrodynamic forces. Gunter et al.[1] developed the force coefficients with a synchronous centered circular orbits assumption for central groove supply with open-end or O-ring sealing. No leakage effect was included for the O-ring. They also showed that the damping capacity of a $2 \pi$-film was twice that of $\pi$-film, but the stiffness was zero for the $2 \pi$-film. San Andrés and Vance [63] examined the coefficients of an open-end damper with 
long axial length executing off-center circular orbits with small amplitude, including the fluid inertia effects. An approximated solution of the finite length damper was obtained by introducing a correction factor to the long damper solution.

The incorporation of SFDs with the form of force coefficients into forced responses analysis is not difficult when using methods such as finite element analysis. One additional step that is needed is the iteration. Since the obtained coefficients are functions of the dynamic behaviors of the journal, presumed coefficients from initial guesses requires iteration to get the desired solution according to current dynamic conditions. Greenhill and Nelson [64] investigated the unbalance response of a multi-shaft turbo fan system supported on SFDs which were considered as $\pi$-film models. They also discussed an iteration scheme to find the damper eccentricity to determine force coefficients for each operating speed, developing a modified secant root-finding method. More recently, Ertas et al.[65] applied this method to a rigid rotor, supported by a gas bearing and squeeze film damper at each end, for unbalance response analysis. Favorable agreement was achieved between simulation and experiments.

\subsubsection{Direct Force Method}

Since the force coefficients method has limitations due to assumptions it is based on, it is not appropriate for use in modeling SFDs not having the assumed conditions. The direct force method is preferable for the analysis of dampers having complex arrangements and operating conditions. It is then possible to not only consider the hydrodynamic forces from the damper, but also include other external forces such as gravity acting on the system. The analysis for this method can be executed in either the time domain or the frequency domain.

\section{The Time Domain}

In the time domain, the damper forces from the integration of pressure distribution are functions of time. No simplifying assumptions on the SFD is required for methods in this domain. The time domain methods are able to analyze not only the periodic solutions but 
also the aperiodic solutions for both steady state response and transient analysis. Taylor [66] found the nonlinear response of a planar rotor supported on a centered-short damper with the fourth order Runge Kutta integration method. The response with $\pi$-film assumption was found to be highly nonlinear, as compared to the almost linear responses under $2 \pi$-film assumption. Cookson and Kossa[19] discussed the transient forced response of an off-centered rigid rotor. Later, they performed a parametric analysis for the steady state response of a flexible rotor without centering springs, under the influence of different parameters of bearing, gravity and unbalance, mass ratio of bearing to mid-disk, etc.

Li and Hamilton[67], with the Runge Kutta integration method, investigated the transient response of a dual-rotor system with short squeeze film dampers. They introduced an intershaft damper which was found to produce an instability threshold speed with non-synchronous whirl. Sykes and Holrnes [21] documented the effects of bearing misalignment on nonlinear force response through numerical integration. Their predicted steady state orbits of the damper correlated favorably with experimental results. The jump phenomenon under different cavitation pressures were also captured by the integration method.

Zhu [22], in their discussion of different methods for seeking multiple solutions of a numerical example, verified the suitability of a numerical integration method using a forth order Runge Kutta with constant time step (to reduce computation time). More recently, Inayat-Hussain[68] studied the bifurcation behaviors of a simple flexible rotor with numerical integration; the occurrence of $2 X, 4 X$ and quasi-periodic vibrations varied with speed parameters.

\section{The Frequency Domain}

The methods in the frequency domain are usually applied to the systems having periodic or quasi-periodic responses. The popular methods include Harmonic Balance Method, Trigonometric Collocation Method and Hybrid Numerical Method.

Harmonic Balance Method(HBM)[69] is a method that assumes steady-state motions can be represented in harmonic form as equation (2.1), which has several fundamental 
harmonics associated with rotor running speed.

$$
\mathbf{x}(\mathbf{t})=\mathbf{X}_{0}+\sum_{i=1}^{k}\left[\mathbf{X}_{c i} \cos \left(\omega_{i} t\right)+\mathbf{X}_{s i} \sin \left(\omega_{i} t\right)\right]
$$

where,

$$
\omega_{i}=i \omega / v=i \Omega
$$

Equation (2.1) indicates that there are $k$ harmonics as fundamental frequencies composing the steady state response of a system with $n$ Degree of Freedoms(DOFs). $\mathbf{X}_{0}, \mathbf{X}_{c 1}, \ldots, \mathbf{X}_{c k}$ and $\mathbf{X}_{s 1}, \ldots, \mathbf{X}_{s k}$ are the $(2 k+1) n$ unknown Fourier coefficients to be determined by substituting the above presumed harmonic form into the equations of motion (in frequency domain), together with proper iteration procedures such as the Newton-Raphson method[70] and Broyden method[71]. More details about the harmonic balance are presented in chapter 5 .

Shiau and Jean [23] used the HBM to obtain periodic force responses of large order nonlinear systems; sub and super harmonic components were included in their analysis. Similarly, Hahn and Chen[24] applied the method to a multi-DOF rotor-bearing system on SFDs; the total $(p+q)$ equations of motion were separated into linear and nonlinear parts. A condensation procedure was adopted first to reduce the system equations into a form only related to nonlinear components, and the unknown coefficients $\mathbf{X}_{c k}^{q}, \mathbf{X}_{s k}^{q}$ associated with these nonlinear components were then solved with iterative procedures before getting unknown coefficients $\mathbf{X}_{c k}^{p}, \mathbf{X}_{s k}^{p}$ related with $p$ linear components of the system.

Bonello et al.[25] introduces a modified form of HBM into the analysis of nonlinear modeling of rotordynamic systems with SFDs. The relations between displacements and forces were expressed in terms of receptance matrices instead of equation of motion as traditional HBM. The forced responses at the location of nonlinear dampers were first solved iteratively before obtaining the displacements of the linear part. An open-end squeeze film damper with centered groove supply was modeled as two identical film lands with the consideration of $\pi$-film cavitation. The pressure profile from short bearing assumption was also adopted for integrating hydrodynamic forces. 
Trigonometric Collocation Method(TCM) has the same displacement form as $H B M$. The solutions are approximated by finite trigonometric series with fundamental frequency $\Omega$ as shown in equation $(2.1)$, which also has $(2 k+1) n$ unknowns to solve. The collocation method essentially requires the form in equation (2.1) to identically satisfy a specified number of time points $(M)$ within one period, and substitutions into equation of motion gives $M * N$ equations. Nataraj and Nelson [26] chose $M$ time points evenly in a period $[0, T]$ such that,

$$
t_{i}=\frac{i T}{M}, i=0,1, \ldots,(M-1)
$$

Then the state variable $x_{i}$ can be related with unknowns to the trigonometric series as in equation $(2.1)$ at the collocation points. To solve the $(2 k+1) n$ unknowns, $M$ is no less than $(2 k+1)$. Large order systems were modeled separately as linear and nonlinear parts, like the $H B M$. Discrete modeling method such as finite elements was employed for the linear part. The connectivity matrix was specified for the connection between linear and nonlinear parts; and the equations of motion were then reduced to the degree of linear parts only. Zhao et al.[27] applied the TCM to a 6-DOF symmetric rotor supported on two identical short SFDs with a $\pi$-film cavitation model.

Hybrid Numerical Method is the combination of $H B M$ and $T C M$, containing the merits of both methods as documented by Hwang and Shiau[72, 73, 74]. The displacement forms were taken as generalized polynomials, which was incorporated into the equation of motion from the principle of virtual work, instead of the finite element method[75]. The equation of motion of the linear part was then transferred from physical coordinates into generalized modal coordinates, as were the nonlinear forces from the dampers. Then, the generalized modal state vectors were expressed in Fourier series as $H B M$, and the collocation technique in $T C M$ was applied to get the solution at the final step. 


\subsection{Stability Analysis}

\subsubsection{Force Coefficients Method}

Similar to the forced response analyses using force coefficients method, the stability analyses for this method are also applied to dampers with presumed conditions such as short bearing precessing centered circular orbits(CCOs)[58, 60, 76]. Gunter et al.[1], modeled a damper under a synchronous CCOs assumption and carried out the stability analysis of a simple flexible rotor by evaluating a 12th-order characteristic equation of the system. Similarly, Rabinowitz and Hahn[58] examined the stability behavior of a symmetric rotor system using the Routh criterion, resulting in stability threshold maps for dampers executing CCOs.

With the same assumed orbit forms as [1] and [58], McLean and Hahn[60] studied the stability of a multi-mass flexible rotor bearing system by linearizing the damper forces around the equilibrium positions. To save computational effort, the analysis was carried out under a rotating Cartesian reference frame. They also illustrated the zero frequency stability map for a system with one damper and studied the stability of a flexible rotor adopted by [58], showing unstable behaviors for small bearing parameters at high eccentricity ratio.

\subsubsection{Direct Force Method}

The Floquet theory in the time domain has been widely used for stability analyses using the direct force method, even for the frequency domain methods such as $H B M[25,24]$ and $\operatorname{TCM}[77,27,74]$. Zhao et al.[77] applied the Floquet theory to a rigid rotor supported on a short damper with $\pi$-film assumption. A perturbed equation of motion, similar to [60], was obtained before applying the Floquet theory. The approximation calculation of the transition matrix was discussed using a discrete method developed by Hsu[78]. With the theory, Zhao et al. illustrated the change of the leading eigenvalue's amplitude subject to different rotating speeds, and examined unstable regions corresponding to the jump areas 
indicated by unbalance responses from TCM. The Floquet theory found an unstable region right after jump-down area, which could not be determined directly from TCM.

Similarly, by using TCM and Floquet theory, Zhao et al.[27] investigated the stability of a simple rotor system under both concentric and eccentric orbits. The stability of the eccentric operating condition has similar behavior as the concentric when the operating speed is not close to the first pinned-pinned critical speed. Within the region of the critical speed, the system became unstable for eccentric operation. Since two discrete points were found in the Poincaré map, 0.5-subharmonic motions were also observed. In other words, [27] indicated that the static loads can only change the system stability behavior near pinned-pinned critical speeds, but have little effects for speeds outside this region.

Using the Floquet theory, Shiau et al.[74], carried out the stability analysis of a rigid rotor as in [79] and a flexible system with four rigid disks. The increase of unidirectional side load was found to improve the stability of the rigid rotor within the range below the second critical speed, similar to the results documented in [80]. When above this critical speed, larger side loads produced a more unstable system and half-subharmonic motions appeared as well. The other investigation of a flexible rotor having two centered SFDs indicated that the first harmonic associated with static offset dominated the system behaviors and the stability region of the system narrowed down as speeds increased.

\subsection{Summary}

At the component level, many experiments have been performed to investigate SFDs' features, subject to cavitation, fluid inertia, different end seal and supply and discharge conditions. The existing analytical models of dampers under these conditions are not guaranteed to generate accurate predictions. Improvements in these models are needed for better simulation, especially for the considerations of bubbly oils, piston rings seal and holes for supply and discharge. 
At the system level, the assumptions for the force coefficients method limit its application to simplified SFDs only, which can not represent the dampers with complex arrangements in practical applications. The direct force method provides one solution to handle practical dampers, but the work in the past usually focuses on rigid rotors or simple symmetric flexible systems with ideal dampers. It fails to consider realistic rotor bearing systems such as aero-engines. There are several important factors neglected in the rotordynamic analysis of previous work, including unidirectional loads and gyroscopic effects. In addition, the widely used Floquet theory for stability analysis in the frequency domain is not computationally efficient, since the method requires a transformation from the frequency domain to the time domain at each iteration step. Other methods are necessary to achieve more efficient calculation for complex rotor bearing systems on SFDs. 


\section{Chapter 3}

\section{SFDs with End Seals and}

\section{Supply/Discharge Conditions}

A major limitation of existing analytical SFD models is their reliance on numerous simplifying assumptions, which do not satisfy the complex operating conditions found in most industrial applications. These complexities are due to end seal mechanisms as well as supply and discharge conditions. In contrast to simple models with open ends, end seals such as piston rings or O-rings are widely used in the real world. Similarly, the experimental evidence of non-zero pressure inside the supply groove shows the inadequacy of the model with zero pressure assumption for the groove. More realistic operating conditions must be considered for accurate predictions.

The main purpose of this chapter is to develop a comprehensive model for the pressure distributions and damping coefficients that can accommodate the complex boundary conditions that exist in real SFD installations. One widely used end seal condition, the piston ring seal, is considered. The supply and discharge conditions such as holes and grooves are also included. Since closed-form analytical solutions for these complicated operating forms can not be obtained, a numerical method is employed. A major challenge for the numerical methods is satisfying the boundary conditions for SFD practical configurations. This chapter discusses 
the boundary conditions associated with piston rings and hole and groove supply/discharge. The finite difference method is used for the pressure calculation. Corresponding damping capacities are obtained for these complex configurations and compared to the open-end and simplified models.

\subsection{Piston Rings}

Piston rings are widely used in the aero-engine based squeeze film dampers, due to their ability to increase the damping and sustain to wear. The typical arrangement of piston rings for SFDs is shown in Figure 3.1. Although the piston rings are used to reduce the leakage happening at axial ends, the pressure drop does happen at the ends due to the radial clearance $h_{s}$ between the ring and housing, as depicted in Figure 3.1. The larger $h_{s}$ is, the larger end leakage will be, which produces lower pressure inside the damper consequently. The axial end pressure can also be affected by the width of the piston ring $w$, which forms the length of leakage path.

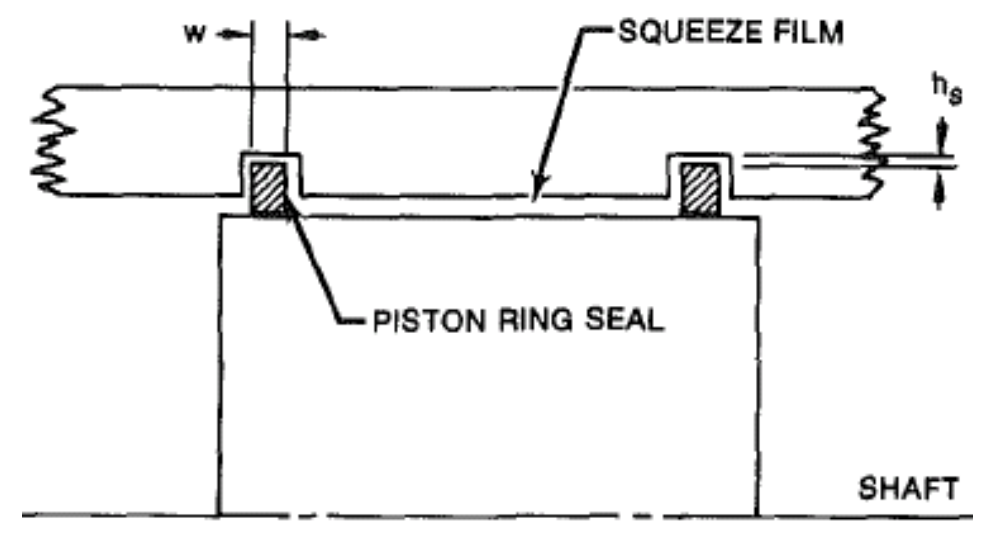

Figure 3.1: SFDs with Piston Rings [2]

The constraint for the leakage can be established using axial flow balance between the flow inside and outside the piston ring. Marmol [2] applied this boundary from piston ring seals. An additional leakage coefficient was introduced to model uncertainties, and the boundary 
condition is formulated as follows,

$$
\frac{h^{3}}{12 \mu} \frac{\partial p}{\partial z}+C_{p} \frac{\left(p-P_{a}\right) h_{s}^{3}}{12 \mu w}=0
$$

When the leakage coefficient $C_{p}=0$, it means there is no leakage at the ends; for $C_{p}=\infty$, it represents open end condition. Note that, the equation 3.1 constrains the flow at the location of $z=L$. For the boundary condition at the other end $(z=0)$, it becomes,

$$
\frac{h^{3}}{12 \mu} \frac{\partial P}{\partial Z}-C_{p} \frac{\left(P-P_{a}\right) h_{s}^{3}}{12 \mu w}=0
$$

Both the finite difference method (FDM) and the finite element method (FEM) are able to solve the pressure distribution of SFDs with piston ring seals. The method of finite difference is chosen due to its simple incorporation for the fluid field having rectangular shape.

\subsubsection{Pressure Distribution Using FDM}

The governing equation for the pressure distribution inside SFDs can be described by Reynolds equation shown below [81],

$$
\frac{\partial}{\partial x}\left(\frac{\rho h^{3}}{\mu} \frac{\partial p}{\partial x}\right)+\frac{\partial}{\partial z}\left(\frac{\rho h^{3}}{\mu} \frac{\partial p}{\partial z}\right)=12 \frac{\partial(\rho h)}{\partial t}
$$

No matter which end seals are used, equation 3.3 gives the basic formulation to solve pressure distribution inside SFDs, although it does not include effects such as fluid inertia and thermal effects. Figure 3.2 shows the fluid film region inside a damper, using the finite difference method. Since the length in circumferential direction is much larger than the damper radial clearance, the pressure variation across the film is neglected. The pressure field can then be represented by a rectangular region, as depicted in the figure. The Reynolds equation above governs all the pressures inside the inner parts of dampers. The pressures at both axial ends are constrained by both equation 3.1 and 3.2 for piston ring seals, while 
the pressure at the two ends in circumferential direction must be equal to each other due to periodic constraints. Hence, the pressure inside the damper can be obtained using these three conditions.

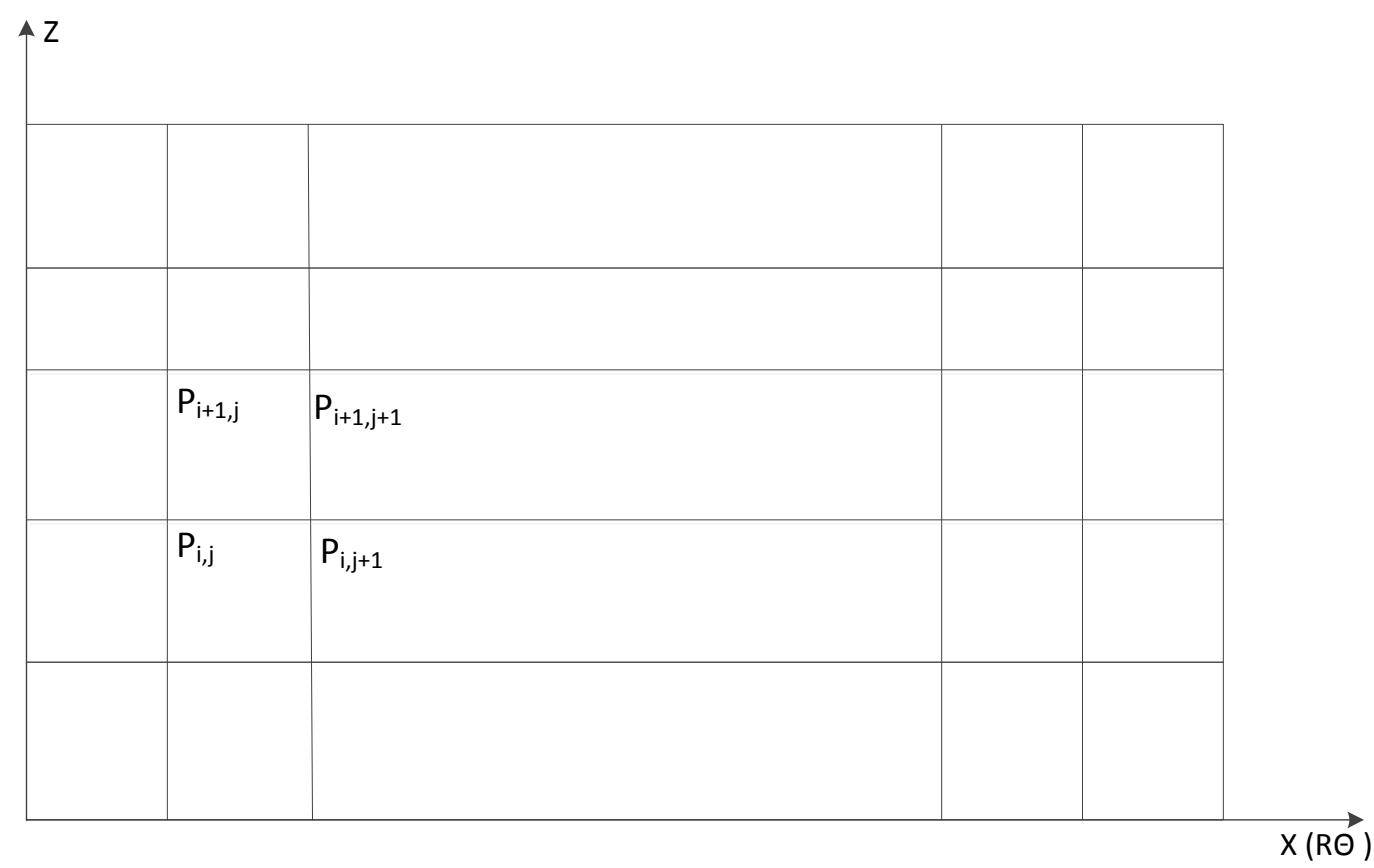

Figure 3.2: Fluid Film Region

To solve the pressure for each part of the damper using the finite difference method, both the Reynolds equation and the end seal boundary conditions need to be discretized. The format of central difference is adopted to approximate the second order derivative, and forward difference for the first order derivative. The Reynolds equation for the finite difference method can be represented as follows using the coordinate in Figure 3.3,

$$
\frac{\rho h_{i, j}^{3}}{12 \mu_{i, j}}\left(\frac{P_{i, j+1}-P_{i, j}}{\Delta X^{2}}-\frac{P_{i, j}-P_{i, j-1}}{\Delta X^{2}}+\frac{P_{i, j+1}-P_{i, j}}{\Delta Z^{2}}-\frac{P_{i, j}-P_{i, j-1}}{\Delta Z^{2}}\right)=\frac{\partial\left(\rho h_{i, j}\right)}{\partial t}
$$

In the equation above, the film thickness $h_{i, j}$ equals to,

$$
h_{i, j}=C+\operatorname{ecos}\left(\theta_{i, j}\right)
$$




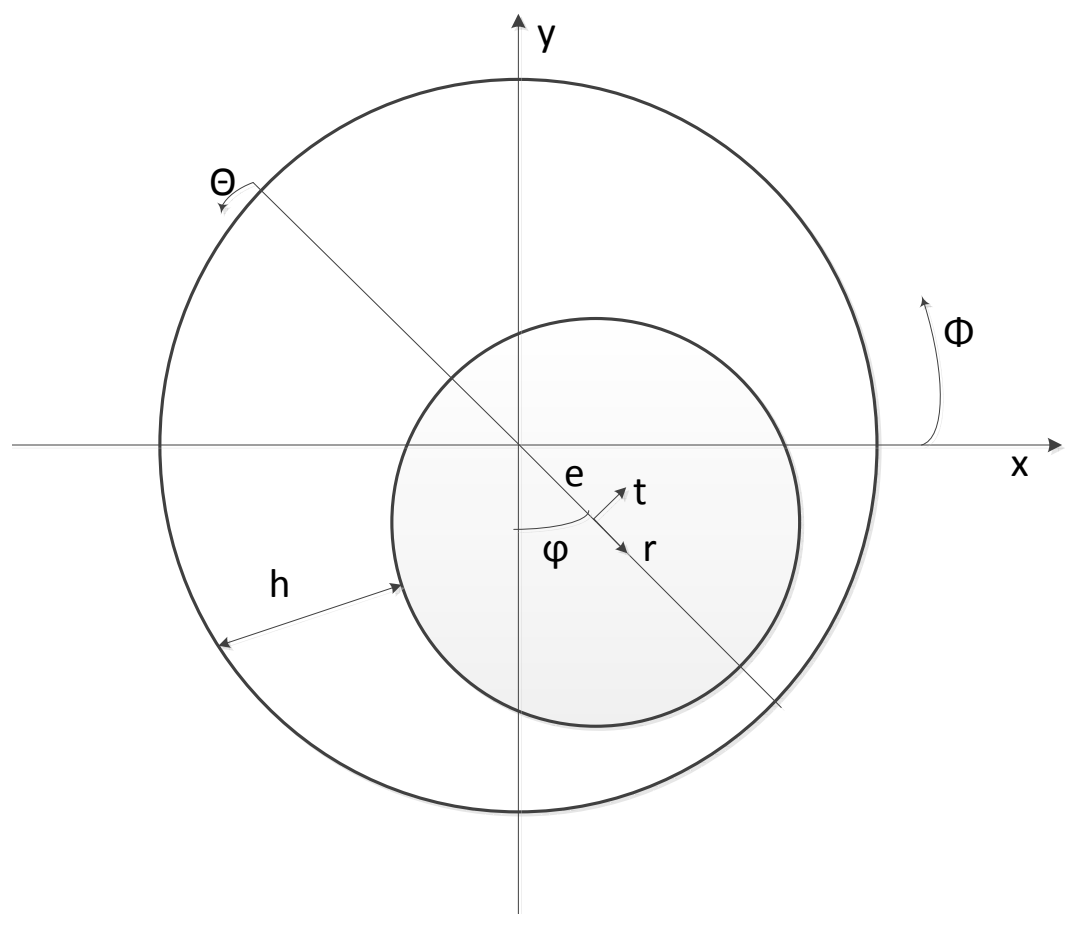

Figure 3.3: SFD Schematic View

Thus, in the line of centers coordinate system, the term on the right side of equation 3.4 gives,

$$
\frac{\partial\left(\rho h_{i, j}\right)}{\partial t}=\dot{e} \cos \left(\theta_{i, j}\right)-e \dot{\theta} \sin \left(\theta_{i, j}\right)
$$

Together with the left hand side of equation 3.4, the complete form of Reynolds equation in finite difference condition can be obtained as,

$$
\frac{\rho h_{i, j}^{3}}{12 \mu_{i, j}}\left(\frac{P_{i, j+1}-P_{i, j}}{\Delta X^{2}}-\frac{P_{i, j}-P_{i, j-1}}{\Delta X^{2}}+\frac{P_{i, j+1}-P_{i, j}}{\Delta Z^{2}}-\frac{P_{i, j}-P_{i, j-1}}{\Delta Z^{2}}\right)=\dot{e} \cos \left(\theta_{i, j}\right)-e \dot{\theta} \sin \left(\theta_{i, j}\right)
$$

Note that, equation 3.7 is valid in the local coordinate system. For the fixed coordinate, a transformation between $\theta$ and $\Phi$ is needed,

$$
\theta=\Phi-\varphi-\frac{\pi}{2}
$$


Similarly, the finite difference forms for the boundary conditions of Equation 3.1 and Equation 3.2 can be expressed as,

$$
\begin{gathered}
\frac{\rho h_{i, j}^{3}}{12 \mu_{i, j}} \frac{P_{i, j+1}-P_{i, j}}{\Delta Z}+C_{p}\left(P_{i, j}-P_{a}\right) \frac{h_{p_{i}}^{3}}{12 \mu_{i, j} w}=0 \\
\frac{\rho h_{i, j}^{3}}{12 \mu_{i, j}} \frac{P_{i, j+1}-P_{i, j}}{\Delta Z}-C_{p}\left(P_{i, j}-P_{a}\right) \frac{h_{p_{i}}^{3}}{12 \mu_{i, j} w}=0
\end{gathered}
$$

The constraint for the periodic boundary is,

$$
P_{i, 0}-P_{i, 2 \pi}=0
$$

Combining all the equations from Equation 3.7 to Equation 3.11 together will solve the pressure at each mesh grid to form pressure distributions of a damper with piston ring seals.

\section{A Damper with Piston Ring Seals}

The example damper has axial length $L=0.4$ inch, radius $R=1.5$ inch and radial clearance $C=2$ mil. The running speed of the journal is $2,000 \mathrm{rpm}$ with synchronous precession. Figure 3.4 gives the pressure distribution of the damper at the location having eccentricity ratio of 0.1 and attitude angle of $90^{\circ}$. The piston rings have width $w=L / 4$, the radial sealing clearance of $h_{s}=C / 2$ and $C_{p}=2.5$. The maximum pressure is around $16 \mathrm{psi}$, and the minimum around 13 psi.

Figure 3.5 shows the comparison of maximum axial pressure among piston rings with different axial thickness. The variation of the thickness has the range from $L / 10$ to $L / 4$. As depicted by the figure, the piston ring with $L / 4$ gives the highest pressure, and the pressure drops linearly with decreasing thickness. Note that the maximum pressure for each case is not present at the middle of axial direction. This happens due to the presence of a central groove at the middle, which has a radial clearance of 8 mil, four times the damper radial clearance of 2 mil. This observation indicates that the pressure inside the groove is not always zero 


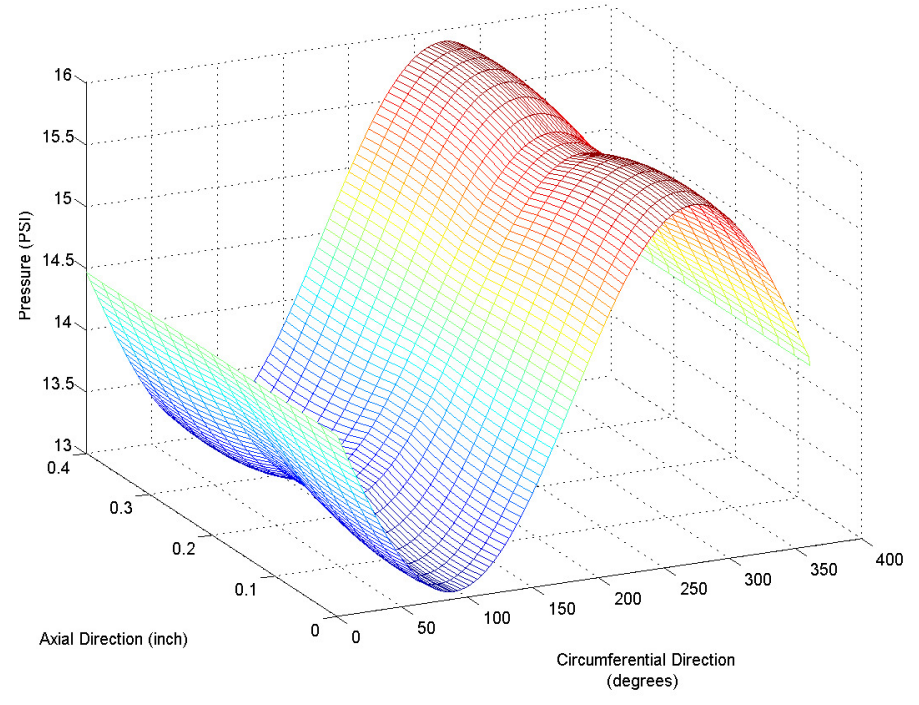

Figure 3.4: Pressure Distribution of a Damper with Piston Ring

and can be the same order of the pressure from the nearby film land. More discussions about this phenomenon is included in part 3.3.

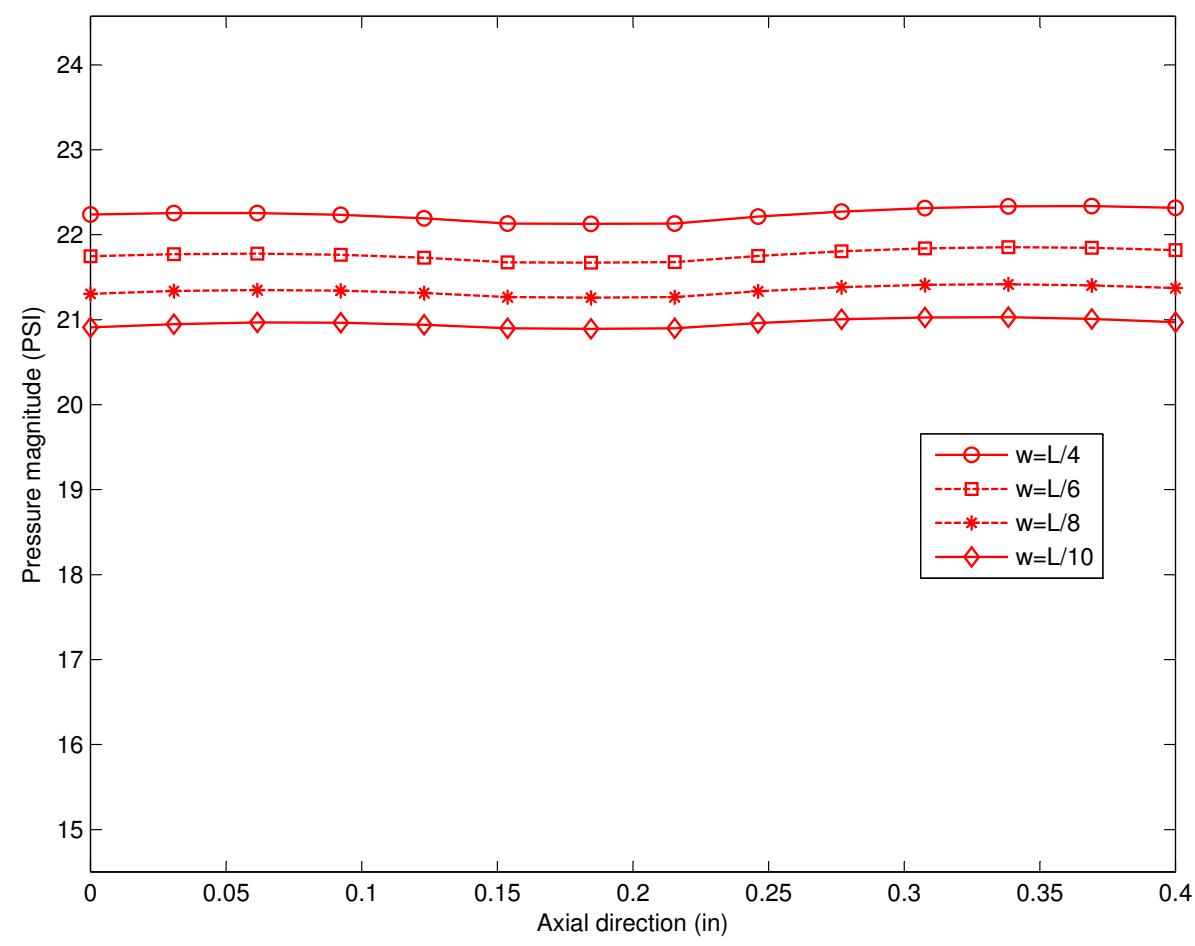

Figure 3.5: Pressure Comparison in Axial Direction

The pressure comparison among dampers with different sealing clearances is depicted in 
Figure 3.6. The pressure change due to the varying clearance is much more obvious than that of the varying axial thickness, which indicates the pressure is much more sensitive to the radial sealing clearance than to the seal axial thickness. The increase of radial clearance, on the other hand, the larger the seal leakage flow which decreases the damper pressure.

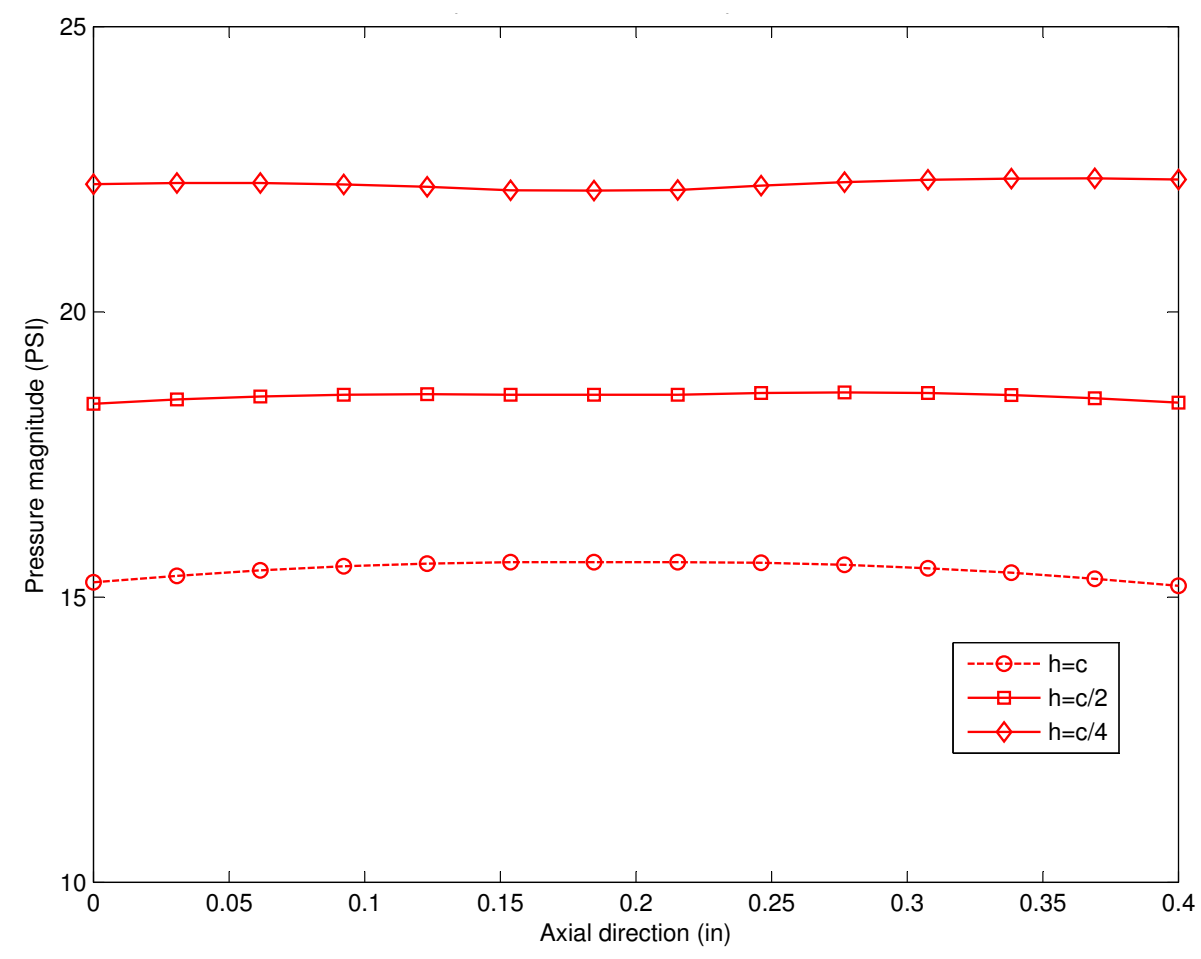

Figure 3.6: Pressure Comparison having Varying Radial Sealing Clearance of Piston Ring

As indicated by equation 3.1 and 3.2 , the leakage coefficient $C_{p}$ plays another important role in the pressure generation. The coefficient counts for the uncertainties between the model given in Figure 3.1 and the realistic setting corresponding to the model. These uncertainties include the surface properties of leakage path and friction between the piston rings and the journal. Benchmark is necessary to give accurate prediction using equations 3.1 and 3.2.

Figure 3.7 shows the axial pressure distribution with different values of $C_{p}$. As depicted by the figure, a high leakage coefficient reduces the pressure within the damper. When $C_{p}$ equals to 100, the end pressure next to the seal is almost the same as the ambient pressure (14.5 psi). It is also found that the changes of pressure is nonlinear with linear change of $C_{p}$. The pressure is more sensitive to the $C_{p}$ below 0.5 than other higher values. The overall 
changing range of pressure is about $100 \mathrm{psi}$ for $C_{p}$ from 0.1 to 100 . The significant change happens for $C_{p}$ between 0.1 and 0.5 , which is about 60 psi. This large variation indicates the important effects of the leakage coefficient on pressure generation and the need of calibration on the coefficient for accurate pressure predictions.

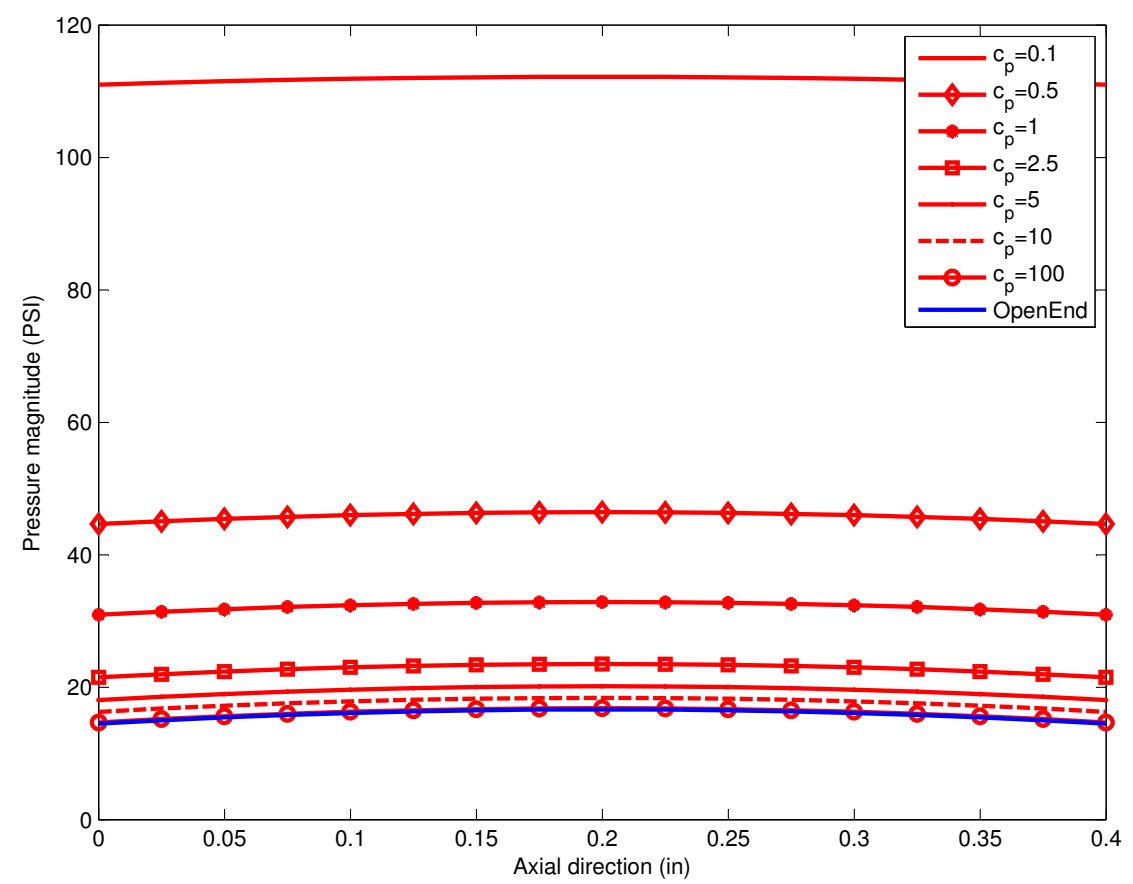

Figure 3.7: Pressure Comparison with Different Leakage Coefficients

\subsubsection{Force Coefficients of SFDs with Piston Ring Seals}

The previous section discussed the pressure distribution of SFDs with piston ring seals. To have a better understanding of dynamic properties of this kind damper, the study of its force coefficients is necessary. Since SFDs can not provide forces without journal precession, the coefficients here mainly refer to the speed dependent coefficients - the damping coefficients, not the stiffness. Then, the damper forces in polar coordinate (as shown in Figure 3.3) are,

$$
\begin{aligned}
& F_{r}=C_{r t} V_{t} \\
& F_{t}=C_{t t} V_{t}
\end{aligned}
$$


The above damper forces in the polar coordinate can be transferred to the Cartesian coordinate using following equation,

$$
\left[\begin{array}{l}
F_{x} \\
F_{y}
\end{array}\right]=\left[\begin{array}{cc}
\sin \varphi & \cos \varphi \\
-\cos \varphi & \sin \varphi
\end{array}\right]\left[\begin{array}{c}
F_{r} \\
F_{t}
\end{array}\right]
$$

Note that, equation 3.12 excludes the inertia terms and assumes a circular orbit of journal motion. With these two presumptions, the damping terms could be obtained by perturbation method as follows,

$$
\begin{aligned}
C_{r t} & =\frac{\Delta F_{r}}{\Delta V_{t}} \\
C_{t t} & =\frac{\Delta F_{t}}{\Delta V_{t}}
\end{aligned}
$$

To generalize the results from a specific SFD, the damping coefficients are non-dimensionalized using following equation,

$$
\begin{aligned}
C_{r t} & =\mu R \frac{L}{D}\left(\frac{R}{c}\right)^{3} \overline{C_{r t}} \\
C_{t t} & =\mu R \frac{L}{D}\left(\frac{R}{c}\right)^{3} \overline{C_{t t}}
\end{aligned}
$$

\section{A Damper with Piston Ring Seals - Dimensionless Damping}

The dimensionless damping coefficients of a damper with synchronous precession speed of 2, $000 \mathrm{rpm}$ are studied. The effects of aspect ratio $L / D$, seal leakage coefficients $C_{p}$ and seal radial clearance $h_{s}$ on the coefficients are compared.

Figures 3.8 and 3.9 give the cross-coupled and direct damping subjects to the change of $L / D$ from $1 / 8$ to 1 , with $c / R=1.8 / 1000, C_{p}=0.5$ and $h_{s}=c$. For each case of $L / D$, the relation between eccentricity ratio and damping coefficients is nonlinear, which is similar to the predictions from short bearing theory[1]. Within the high eccentricity region, SFDs generate more nonlinear damping coefficients than that within the low eccentricity region.

With increasing $L / D$, larger damping is produced, as expected. The damping is more sensitive to the variation below 0.5. Comparing Figure 3.8 with 3.9, it can also be found that 


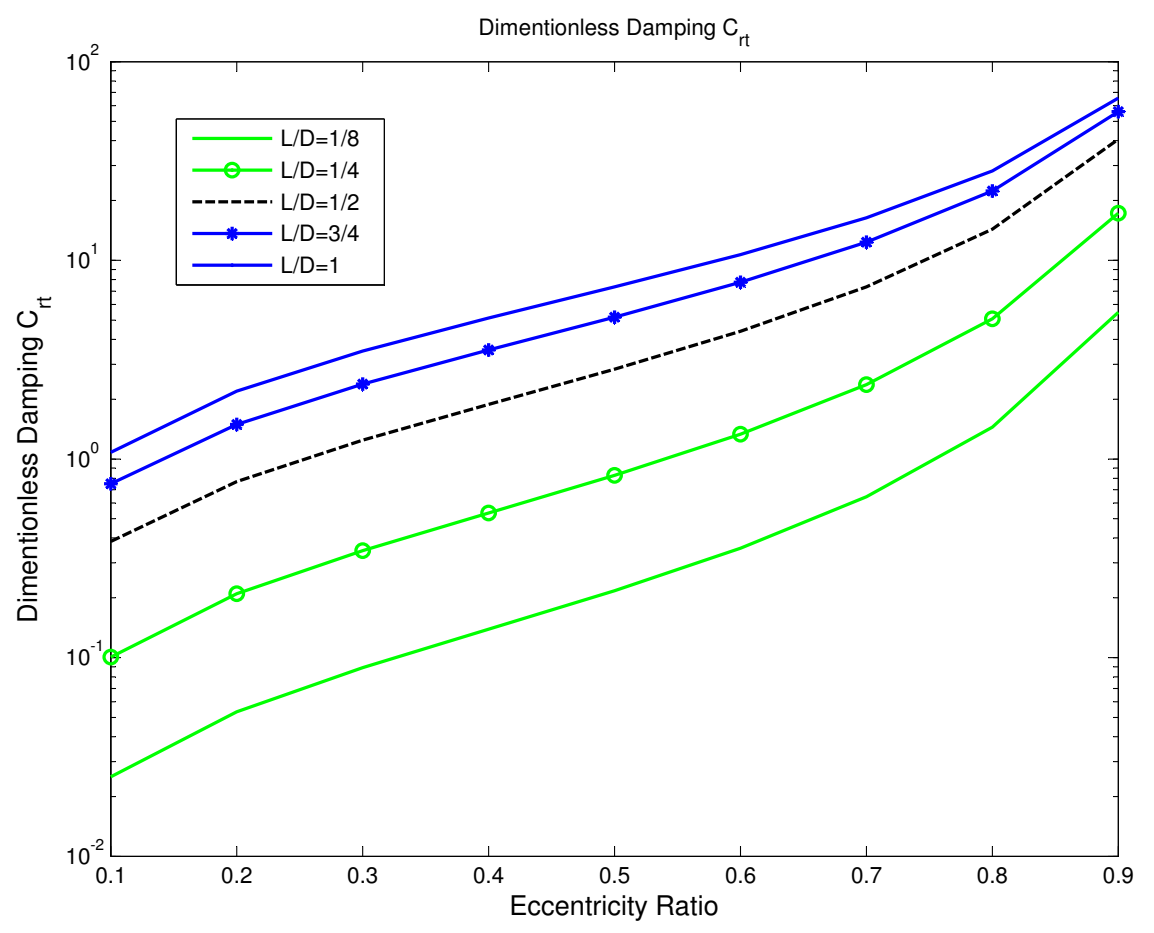

Figure 3.8: $\overline{C_{r t}}$ with Different $L / D$

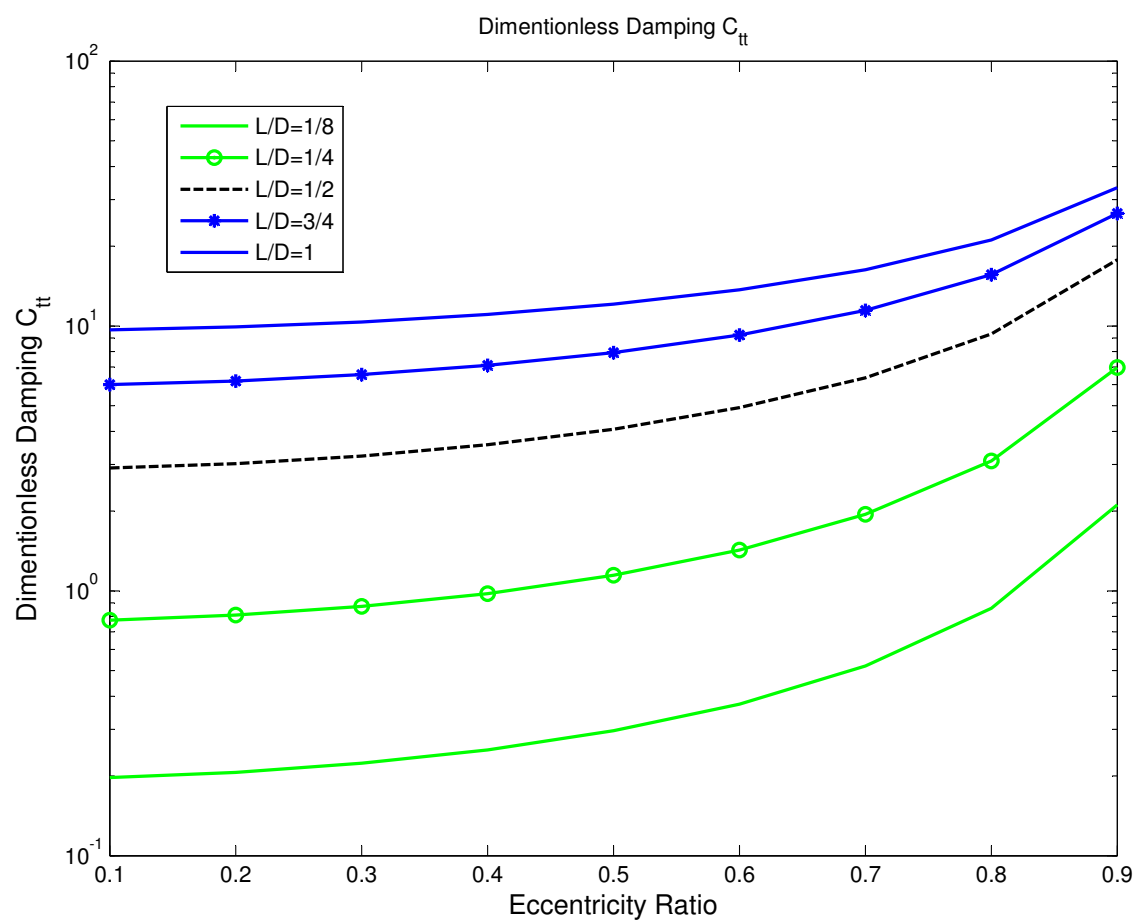

Figure 3.9: $\overline{C_{t t}}$ with Different $L / D$ 
the direct damping is about 10 times higher than the cross-coupled damping with eccentricity ratio less than 0.2. However, the increasing rate of cross coupled damping is larger than that of the direct damping, which results higher value of $\overline{C_{r t}}$ when the eccentricity ratio is above 0.7. Since the cross-coupled damping is equivalent to direct stiffness for SFDs[39], this observation indicates that the effective damping of a SFD is decreasing as eccentricity increases, which could result in a less stable or unstable system.

Figure 3.10 and 3.11 show the effects of leakage coefficient $C_{p}$ on the cross coupled and direct damping, respectively. The changing range of $C_{p}$ is from 0.1 to 100 , which is similar to that shown in Figure 3.7. The $L / D$ is fixed to $1 / 8 . c / R$ and $h_{s}$ are the same as the previous study on the effects of $L / D$. Correlated with the trend of pressure drop given previously, the damping coefficients are decreasing with leakage. The reduction of damping within the range from 0.1 to 0.5 covers about $50 \%$ of the overall damping variation for studied leakage range. When $C_{p}$ is larger than 5 , its variation has little influence on the damping coefficients. Different from the trend subject to varying $L / D$, the tendency of damping variation due to leakage are nearly the same for all the $C_{p}$, which are parallel to each other. This observation is expected since the leakage coefficient only changes the pressure value at damper ends, which does not change the distribution shape of the pressure inside the film land much.

From Figures 3.10 and 3.11, both cross coupled and direct damping of the piston ring sealed SFD are almost the same as value of open-end dampers when $C_{p}$ increases to 100 , which shows the correlation with the pressure distribution depicted in Figure 3.7. However, if SFDs are well sealed by piston rings, they can produce much higher damping than open-end dampers. Taking the $C_{p}=0.5$ for example, both $\overline{C_{r t}}$ and $\overline{C_{t t}}$ are about four times as large as these from open-end cases. This provides one of the reasons for the wide application of piston ring seals in aero-engine dampers.

The radial clearance $h_{s}$ of the piston ring plays another important role on the pressure generation by the damper and on the dynamics of the journal. Tighter assembled piston rings have less leakage. Meanwhile, they give less displacement space of the journal. Therefore, if 


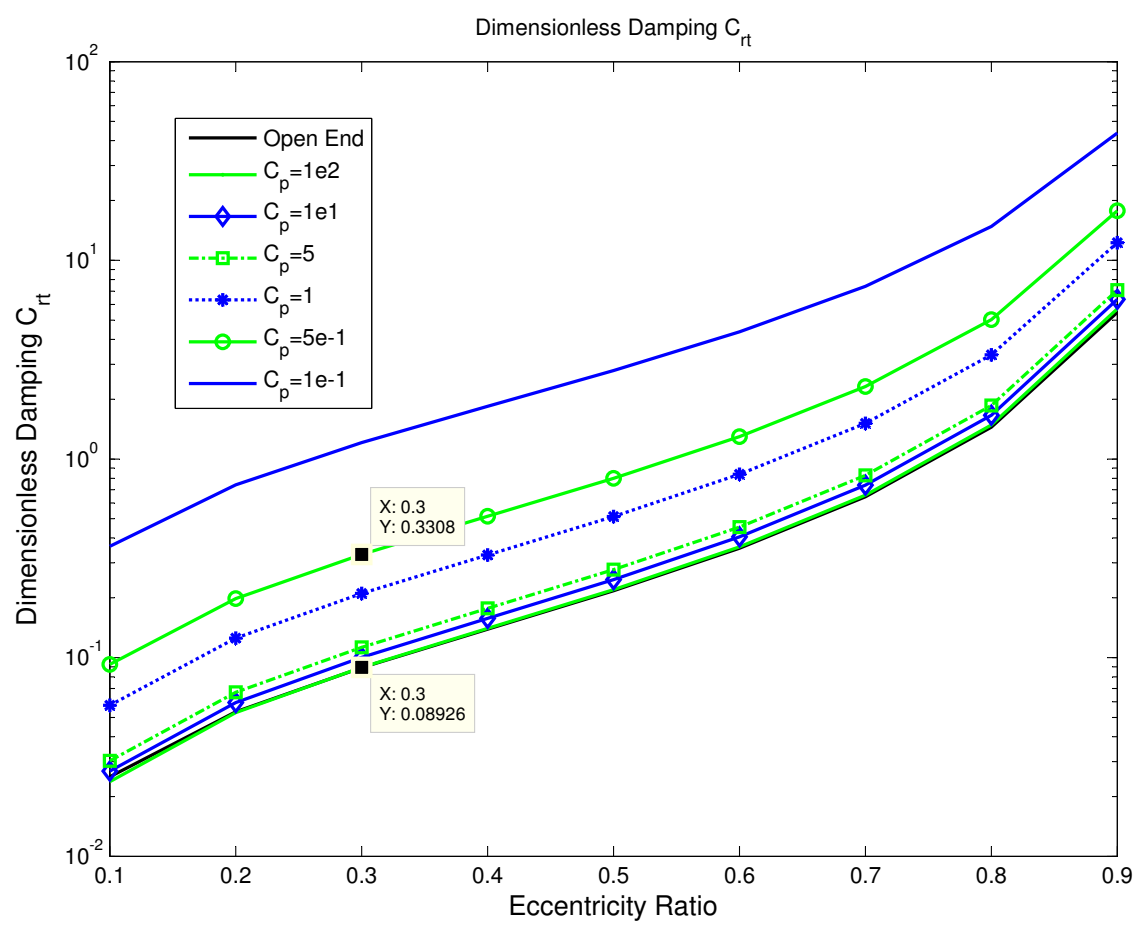

Figure 3.10: $\overline{C_{r t}}$ with Different $C_{p}$

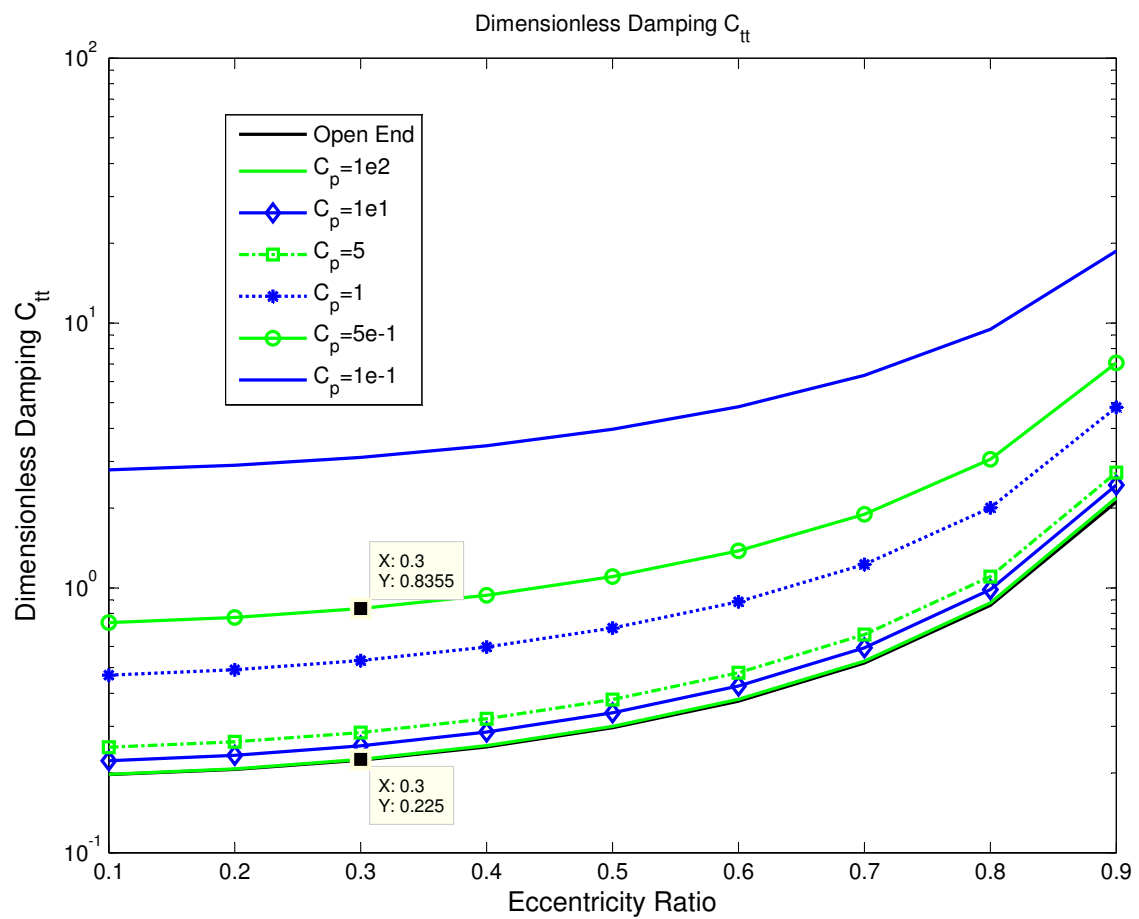

Figure 3.11: $\overline{C_{t t}}$ with Different $C_{p}$ 
the journal has larger vibration amplitude than $h_{s}$, the direct contact between the journal and inner face of the piston rings can happen. This could produce a bilinear effect where the radial stiffness of the ring contributes to the journal behaviors. In the current study, this bilinear effect is neglected. If contact happens, the local ring clearance is considered to be zero and no leakage exists locally.

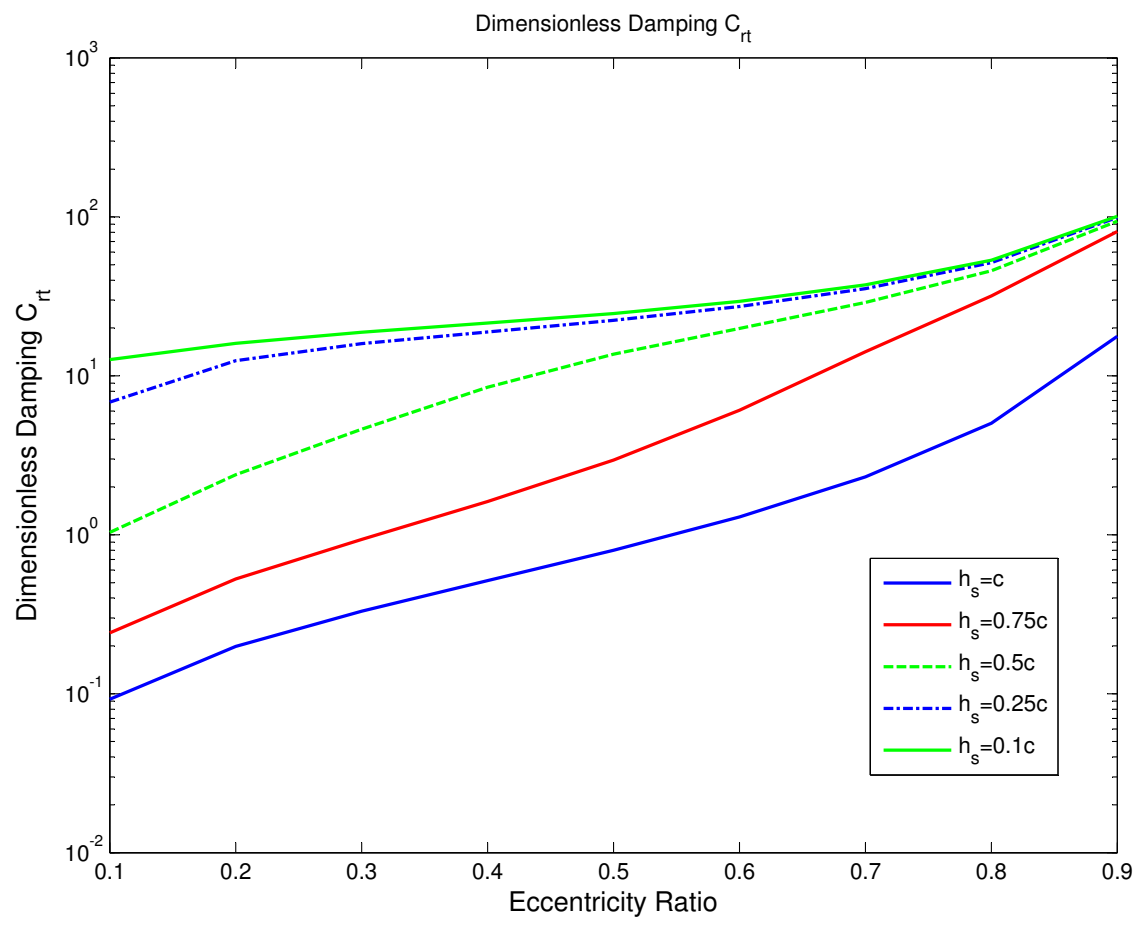

Figure 3.12: $\overline{C_{r t}}$ with Different $h_{s}$

The effects of the piston ring radial clearance $h_{s}$ on the damping coefficients are given in Figures 3.12 and 3.13. For these cases, $L / D=1 / 8, c / R=1.8 / 1000$ and $C_{p}=0.5 . h_{s}$ varies from $25 \%$ to $100 \%$ of the damper radial clearance. From Figures 3.12 and 3.13, a smaller ring clearance $h_{s}$ produces larger damping coefficients, and the tendency is nonlinear. The effect of $h_{s}$ on the damping capacity is decreasing as the clearance becomes tighter. When $h_{s}$ is below $0.25 c$, the cross-coupled damping values are quite close to each other, especially for the region from middle to large eccentricity ratio. The cross-coupled damping also has less damping difference between low and high eccentricity regions, when $h_{s}$ is small. The 
damping difference between the minimum and the maximum for $h_{s}=0.5$ is about 100 , while it is around 88 for $h_{s}=0.1$.

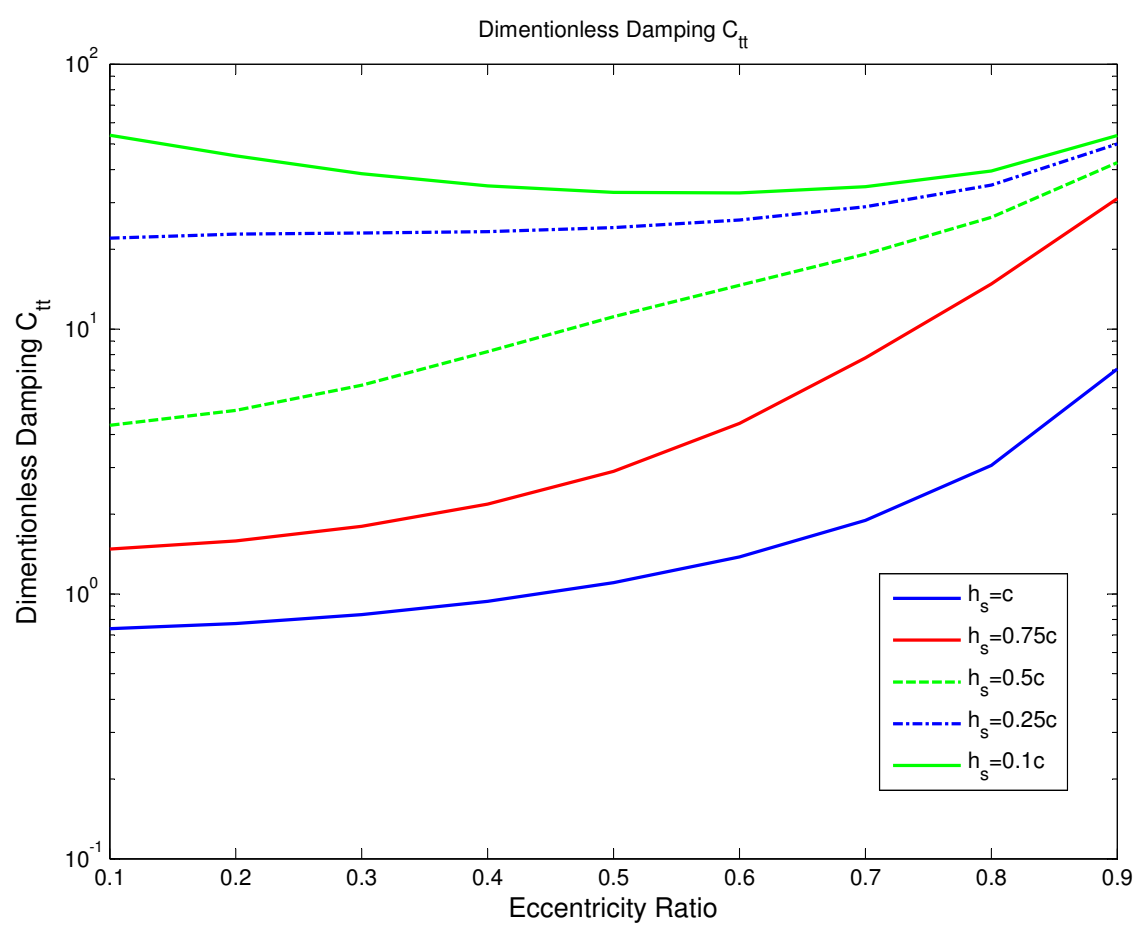

Figure 3.13: $\overline{C_{t t}}$ with Different $h_{s}$

For the direct damping $\overline{C_{t t}}$, as shown in Figure 3.13, although it tends to converge as the journal operates within the high eccentricity region, it behaves differently for the eccentricity ratio between moderate and small value. Its increasing rate is larger than that of cross-coupled damping for small eccentricity. The tendency of $\overline{C_{t t}}$ changes from monotonic increase $\left(h_{s}=0.5\right)$ to parabolic variation $\left(h_{s}=0.1\right)$. For $h_{s}=0.25$, the shape of $\overline{C_{t t}}$ is quite flat over the region with eccentricity ratio below $70 \%$. Within the low eccentricity region, the increasing rate of direct damping with $h_{s}$ is larger than that of cross-coupled damping, which indicates the effective damping is rising for the region. In other words, when the journal is inside the low eccentricity region, tighter clearance of piston rings can result in more stable system. Caution must be paid for this conclusion, since the contact model with bilinear effect is not included in current analysis, not are the thermal effects. 


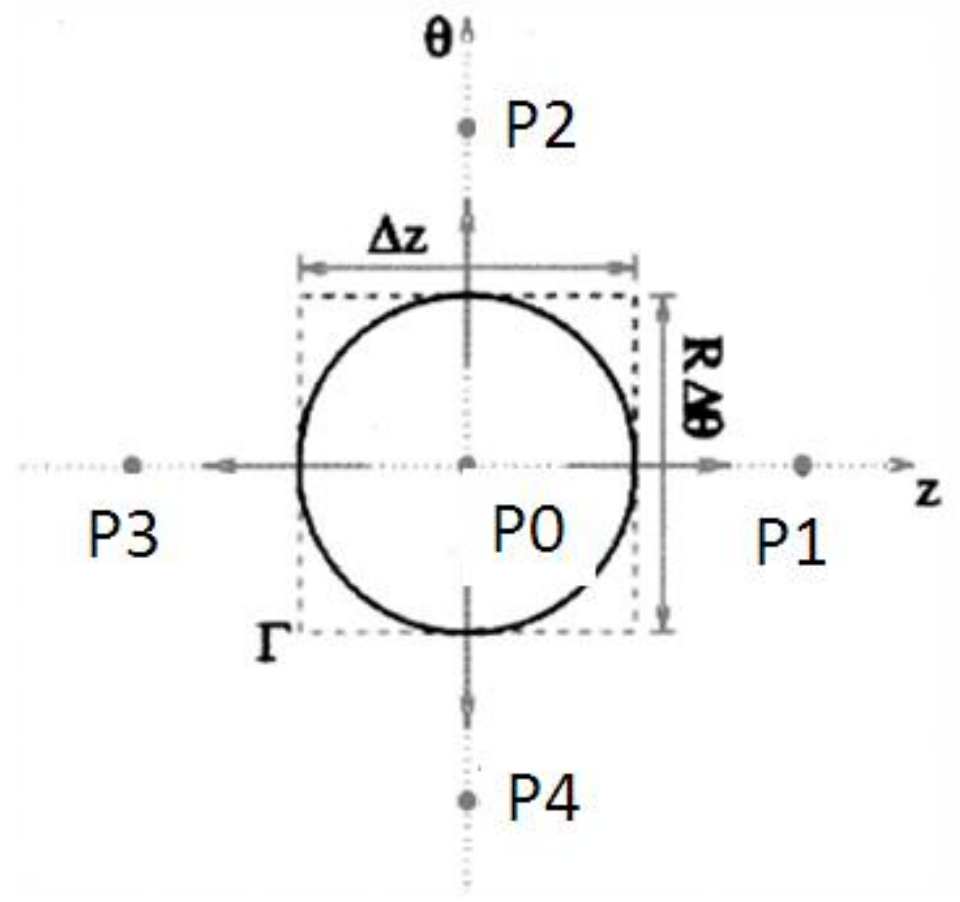

Figure 3.14: Finite Difference Mesh for Hole Supply/Discharge

\subsection{Hole Supply/Discharge}

\subsubsection{Pressure Distribution}

Hole supply/discharge is considered as one way to improve the damping capacity of SFDs [51]. The modeling of this configuration can be established by either the flow balance between outward and inward flow through the hole or the constraint on the pressure (equal to supply/discharge pressure) at the location of holes. The method of flow balance is used in this work, together with the finite difference method. For this method, the inward flow $Q_{\text {in }}$ equals to the outward flow $Q_{\text {out }}$, which is $Q_{\text {in }}=Q_{\text {out }}$. Figure 3.14 gives the mesh around the hole using the finite difference method. Note that, the grid size in both the axial and circumferential directions is larger than the radius of the hole. The flow balance through the hole can be approximated as [2],

$$
C_{h}\left(p_{s}-P_{0}\right)=\frac{h^{3}}{12 \mu}\left(\frac{\partial P_{3}}{\partial x}-\frac{\partial P_{1}}{\partial x}\right) \Delta z+\frac{h^{3}}{12 \mu}\left(\frac{\partial P_{4}}{\partial z}-\frac{\partial P_{2}}{\partial z}\right) \Delta x
$$


The left side of equation 3.16 represents the flow from the supply into the hole, and the right side gives the flow from the hole into damper film land. The finite difference format of the above equation is represented as below. Again, the forward difference is used.

$C_{h}\left(p_{s}-P_{i, j}\right)=\frac{h^{3}}{12 \mu}\left(\frac{P_{i, j}-P_{i-1, j}}{\Delta X}-\frac{P_{i+1, j}-P_{i, j}}{\Delta X}\right) \Delta Z+\frac{h^{3}}{12 \mu}\left(\frac{P_{i, j}-P_{i, j-1}}{\Delta Z}-\frac{P_{i, j+1}-P_{i, j}}{\Delta Z}\right) \Delta X$

$P_{i, j}$ is the pressure at the center of the hole.

\section{A Damper with Hole Supply/Discharge - Pressure Distribution}

The damper chosen here has the same dimension and operating conditions as the one in Part 3.1.1. Besides one piston ring seal at each end, two holes are located at the middle location in axial direction with $\Phi$ of $90^{\circ}$ and $270^{\circ}$ for supply and discharge, respectively. The holes have diameter of 0.1 inch. The pressure of supply and discharge equals to the ambient pressure. Applying the boundary condition in equation 3.17 gives the pressure distribution of the damper as shown in Figure 3.15.

The previous smooth pressure distribution becomes folded with raised and decreased pressure due to supply and discharge holes. Note that, the pressure next to the axial end does not have smooth trend either. It has two peaks near the locations of both supply and discharge hole. This happens due to the short length of the damper in axial direction. The diameter of the hole is $25 \%$ of the damper axial length, which makes the supply/discharge hole dominant in the pressure distribution along axial direction. As the damper increases its axial length, the pressure at axial end becomes smooth, as depicted in Figure 3.16.

The damper in Figure 3.16 has an axial length of 4 inches, with the hole diameter of 0.1 inch unchanged. For this longer damper in axial direction, it gives much higher pressure, $38.38 \mathrm{psi}$, compared to the previous $17.60 \mathrm{psi}$; its pressure distribution along the axial end changes smoothly without sudden variation near the location of supply and discharge. As observed, the abrupt region appears within the range of \pm 0.5 inch around the center of the hole. Outside this area, the pressure distribution becomes polished in both axial and 


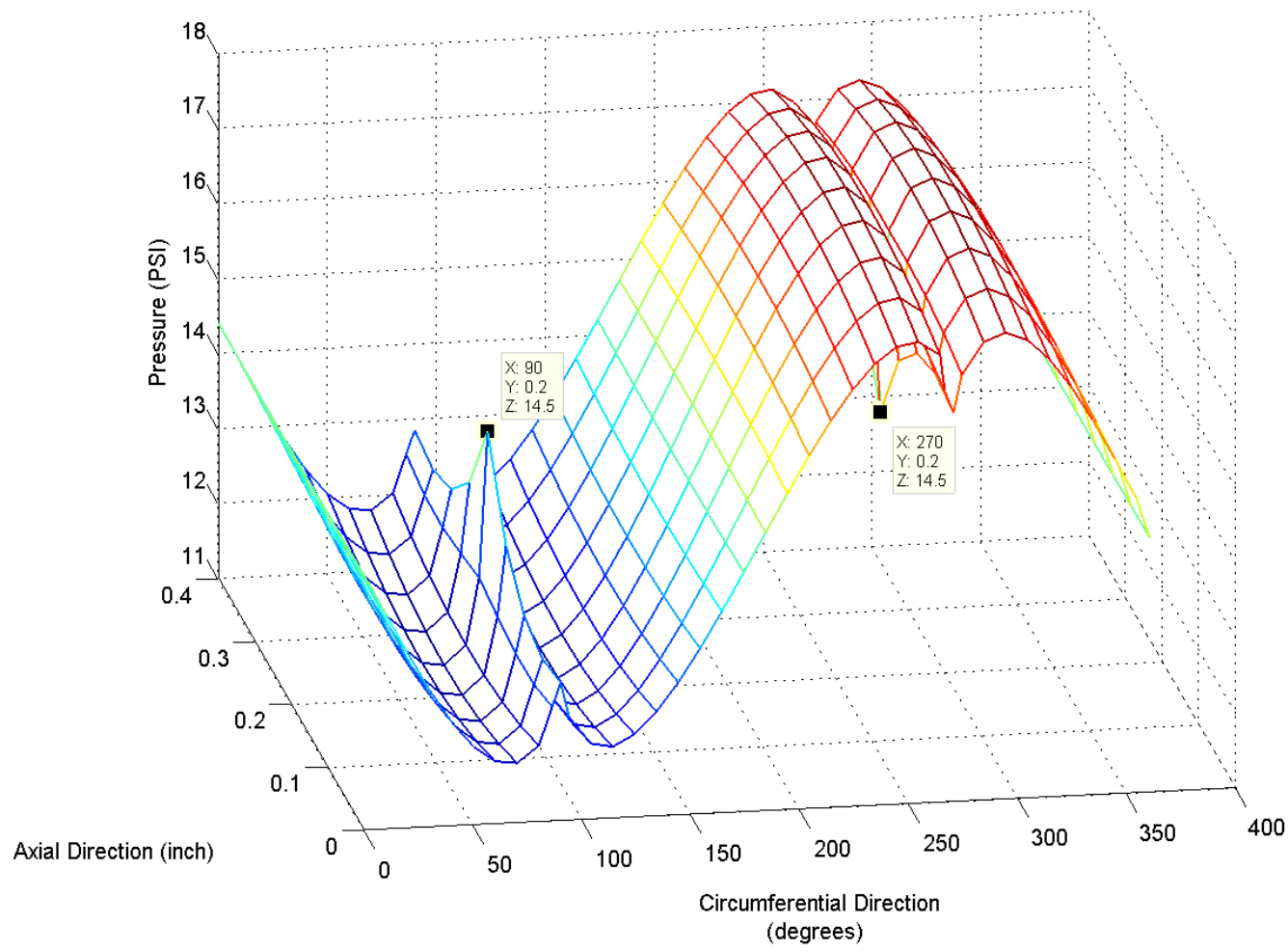

Figure 3.15: Pressure Distribution with Piston Rings Seals and Hole Supply/Discharge

circumferential direction. It is also worth noting that the previous damper has $L / D$ of 0.13 , which is a tenth of the current one. This observation concludes that the hole supply/discharge affects the pressure profile of short dampers much more than that of long dampers, especially for the pressure at axial ends.

\subsubsection{Force Coefficients of SFDs with Hole Supply/Discharge}

To study the damping capacity of SFDs with hole supply/discharge, the same procedure and assumption as in part 3.1.2 are used here. The damping coefficients are non-dimensionalized in the polar coordinate, and synchronous circular centered orbit is presumed for the journal precession. A damper having the same geometric parameters in the previous two parts will be used for the analysis.

\section{A Damper with Hole Supply/Discharge - Force Coefficients}




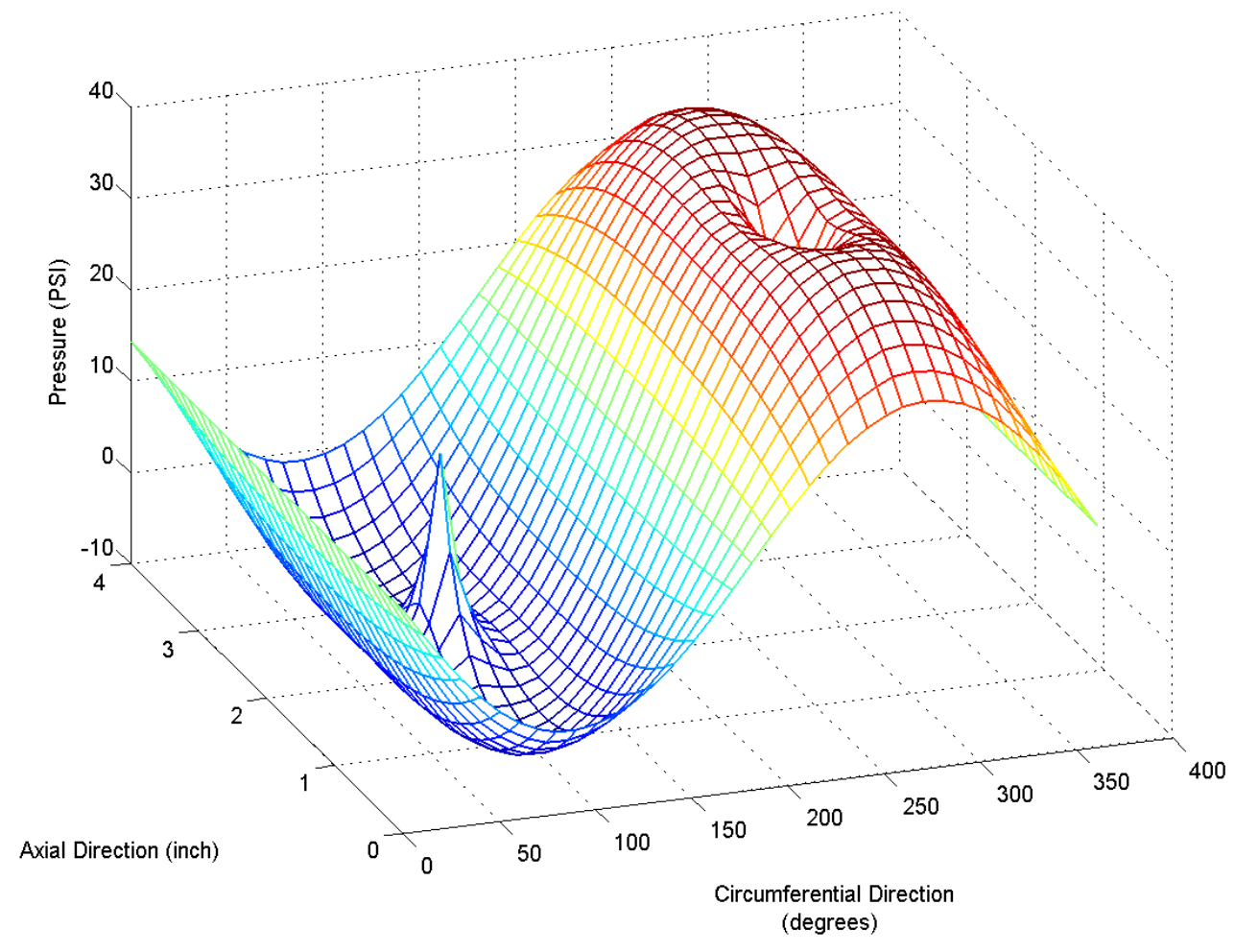

Figure 3.16: Pressure Distribution for Long Damper

The damper is sealed by piston rings, with $C_{p}=0.5, h_{s}=c$ and ring axial width $w_{s}=L / 4$. The effects of supply/discharge pressure through holes on the damping coefficients are investigated. There are two holes with identical diameter of $L / 4$, located at $90^{\circ}$ and $270^{\circ}$ in the circumferential direction and at the middle span in the axial direction. The hole at $90^{\circ}$ is considered as supply hole, and the other is discharge hole. During the investigation, the supply pressure changes from $1 \mathrm{~atm}$ to $40 \mathrm{~atm}$, while the discharge pressure is fixed to ambient pressure. Although the current study focuses on the effects of supply pressure, the results can be applied to the effects of discharge pressure, as the chosen damper has symmetric supply and discharge configuration.

Figures 3.17 - 3.19 give the dimensionless damping for cavitated film. For the cases with cavitation, the rupture pressure equals to the ambient pressure, which is $1 \mathrm{~atm}(14.5$ psi). Figures 3.17 and 3.18 show the cross-coupled and direct damping, respectively. The cross-coupled damping is almost invariant for all the different supply pressures over entire 
eccentricity range, which indicates the supply pressure does not affect the cross-coupled damping. On the other hand, the supply pressure can increase the direct damping term when the supply pressure is below $30 \mathrm{~atm}$, as shown by Figure 3.18.

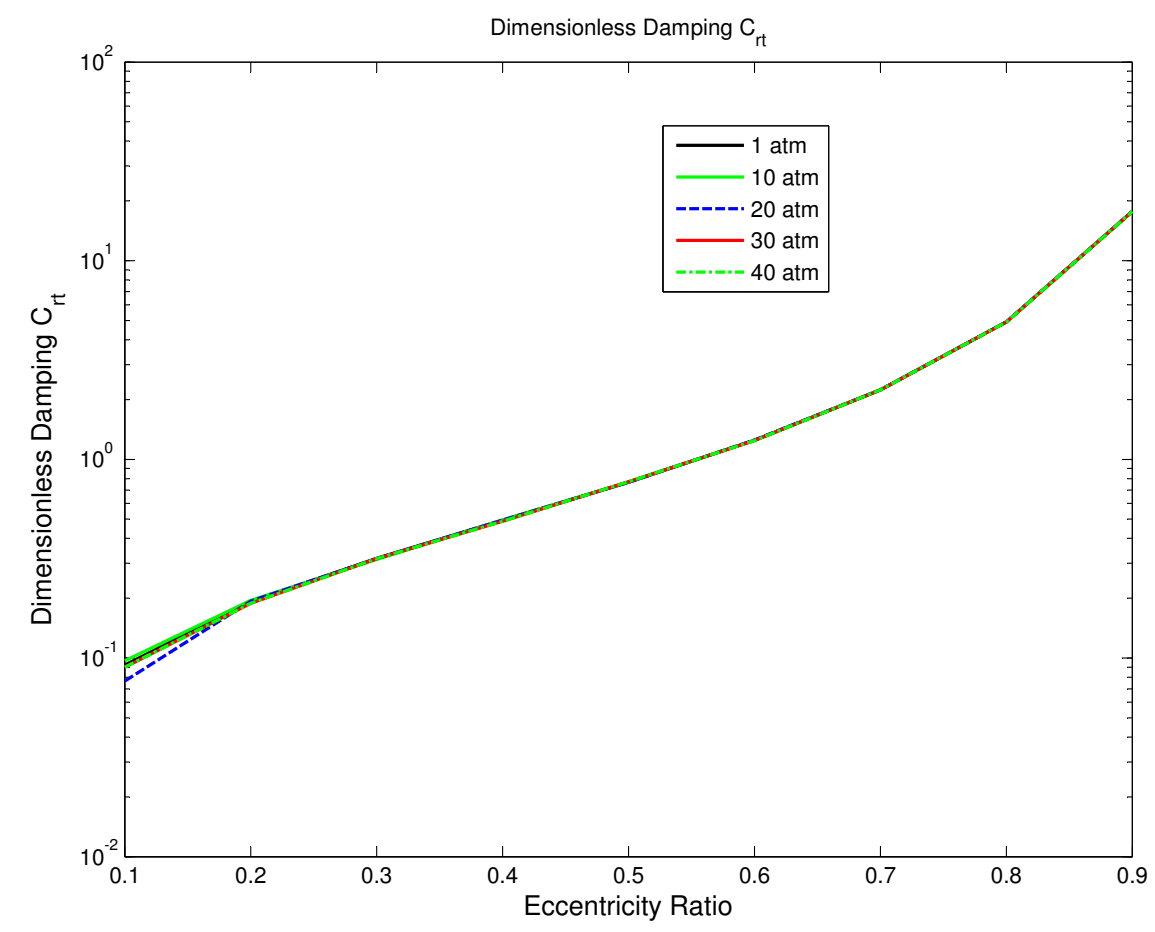

Figure 3.17: $\overline{C_{r t}}$ of Cavitated Film with Varying Supply/Discharge Pressure

However, the linear increase of supply pressure between $1 \mathrm{~atm}$ and $30 \mathrm{~atm}$ does not give linear increase of the damping. The rise from $1 \mathrm{~atm}$ to $10 \mathrm{~atm}$ gives the largest increase. Same amount of pressure increase above 10 atm gives much less damping rise. As shown by both Figures 3.18 and 3.19, the direct damping reaches its limit when the supply pressure is $30 \mathrm{~atm}$ and can not increase further with the pressure, which results in the overlapped distribution when pressure is $40 \mathrm{~atm}$.

With these observations, the increase of supply pressure is shown to be able to improve the effective damping of a SFD within low to moderate eccentricity ratio, therefore, supply pressure plays one of the factors to improve system stability. However, there is a limit of the supply beyond which the direct damping can not be improved further. The studied cases also indicate that the increasing rate of direct damping is decreasing as supply pressure rises, 


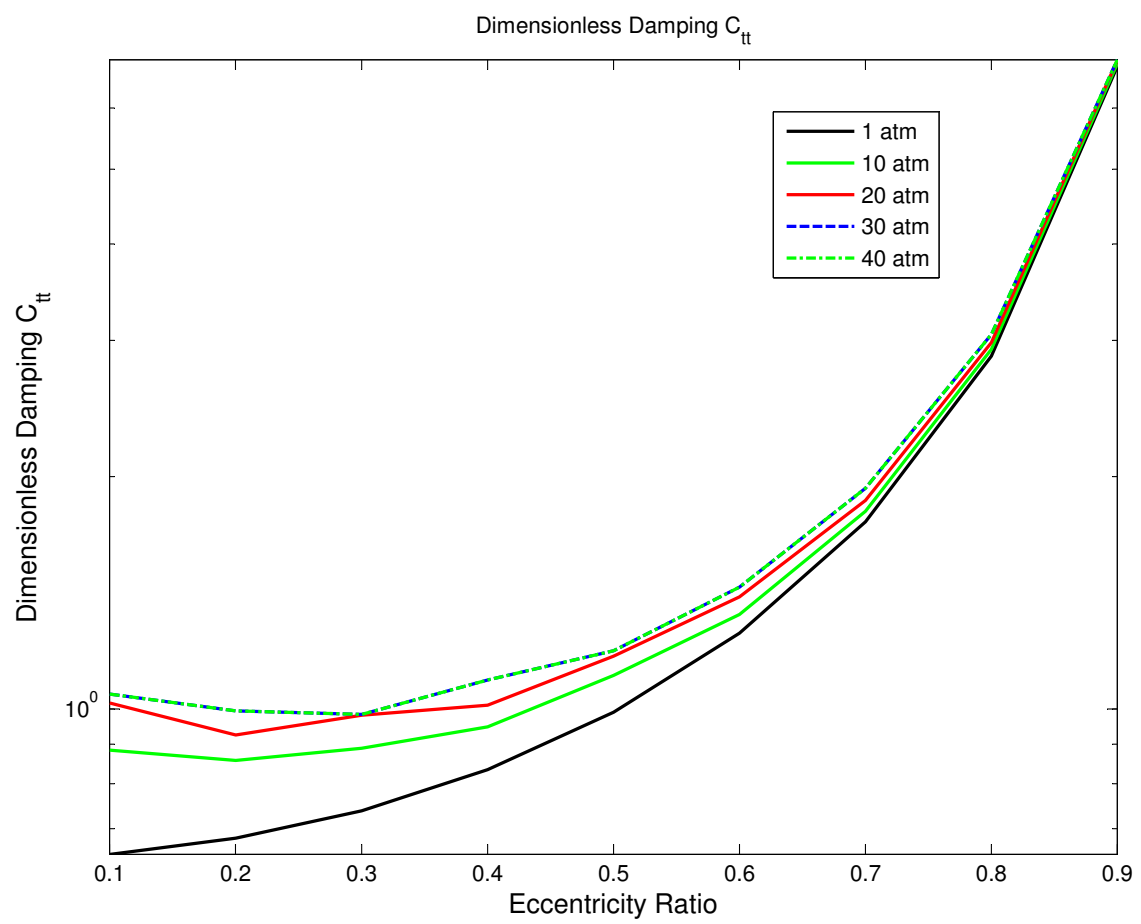

Figure 3.18: $\overline{C_{t t}}$ of Cavitated Film with Varying Supply/Discharge Pressure

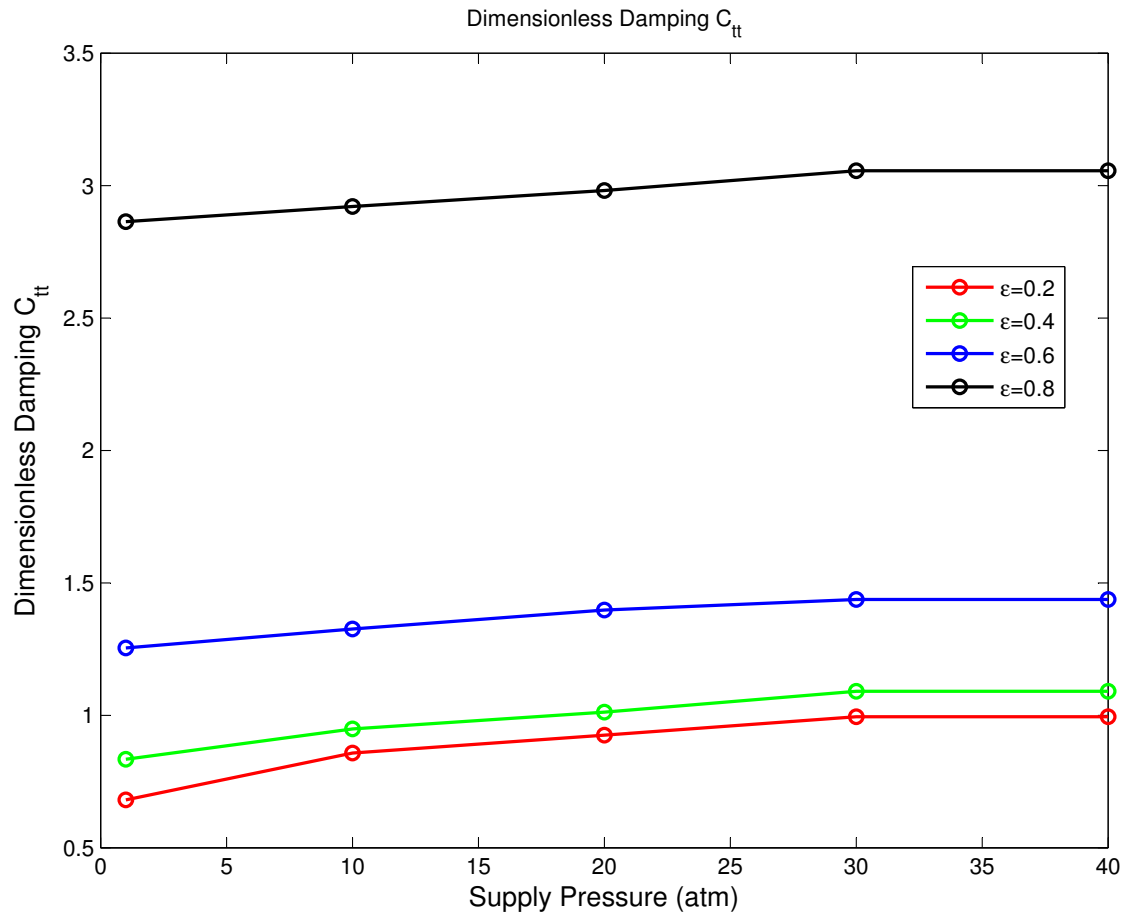

Figure 3.19: $\overline{C_{t t}}$ of Cavitated Film (Fixed Eccentricity) 
as shown in Figure 3.19. The same amount of the supply pressure within low pressure range gives higher damping rise than that within high pressure range.

Table 3.1: Circumferential Location of Holes

\begin{tabular}{c|c}
\hline Number of Holes & Circumferential Location (in degree) \\
\hline 2 & 90,270 \\
\hline 3 & $30,150,270$ \\
\hline 4 & $0,90,180,270$ \\
\hline 5 & $54,126,198,270,342$ \\
\hline 6 & $30,90,150,210,270,330$ \\
\hline
\end{tabular}

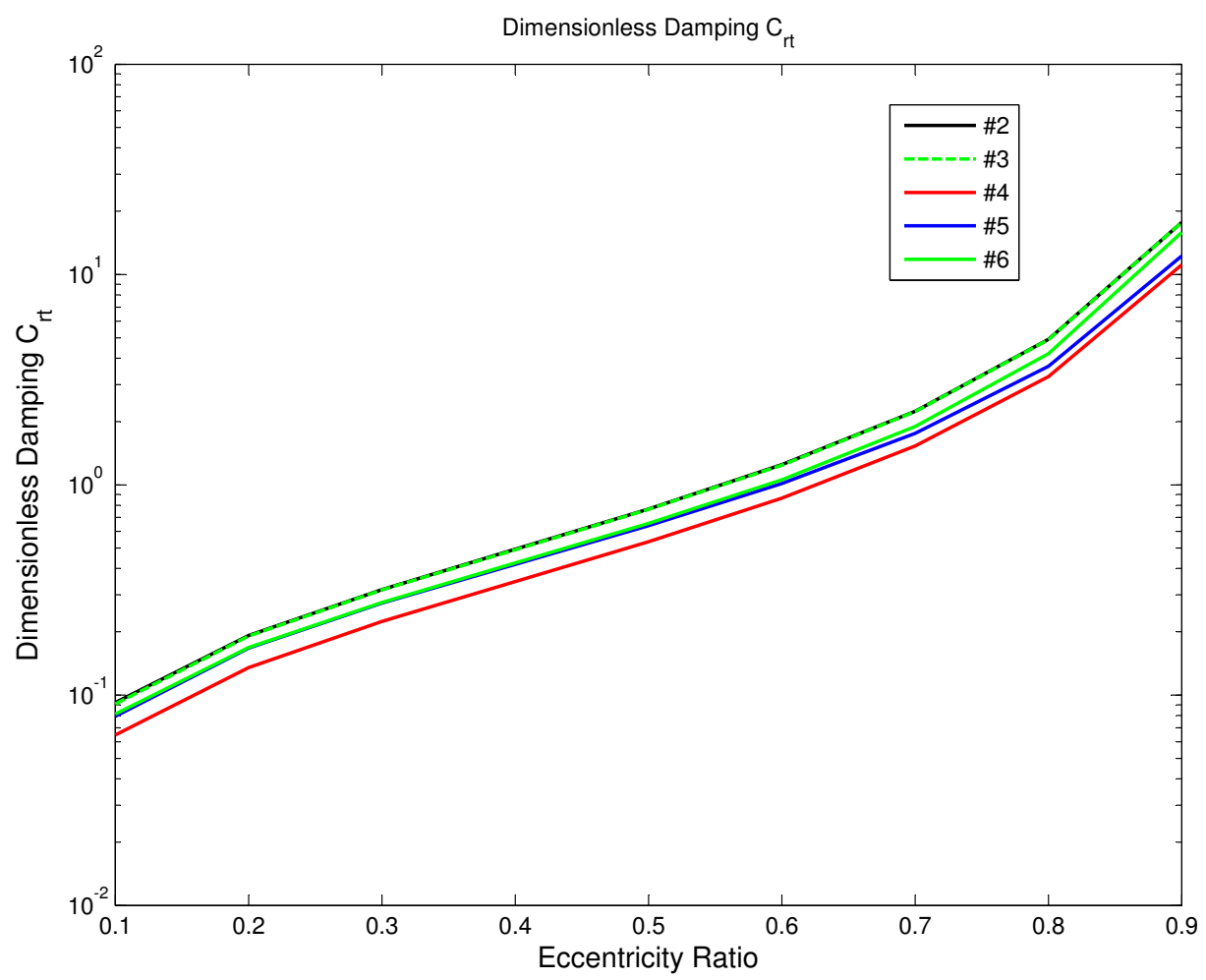

Figure 3.20: $\overline{C_{r t}}$ of Cavitated Film with Hole Number

Figures from 3.20 to 3.23 depict the change of damping capacity of cavitated film subject to varying supply/discharge holes. Five different hole numbers, from two to six, are studied. The circumferential hole location of each case is listed in Table 3.1, and the axial location is fixed to the middle of damper axial length. Current study chooses the location of $270^{\circ}$ as the only discharge hole, no matter how many holes on the damper. Both the supply and discharge pressure for each case are fixed to $1 \mathrm{~atm}$. From Figure 3.20, the hole number is 
found to affect the cross-coupled damping nonlinearly. The number of two holes gives the largest damping, although the case of three holes produces quite similar damping over entire eccentricity. It is interesting to find that the smallest damping comes from four holes, not the case with five or six holes. The five and six holes have quite similar damping for eccentricity lower than $60 \%$. Then they start to split, the case of six holes gives larger damping with higher eccentricity ratio.

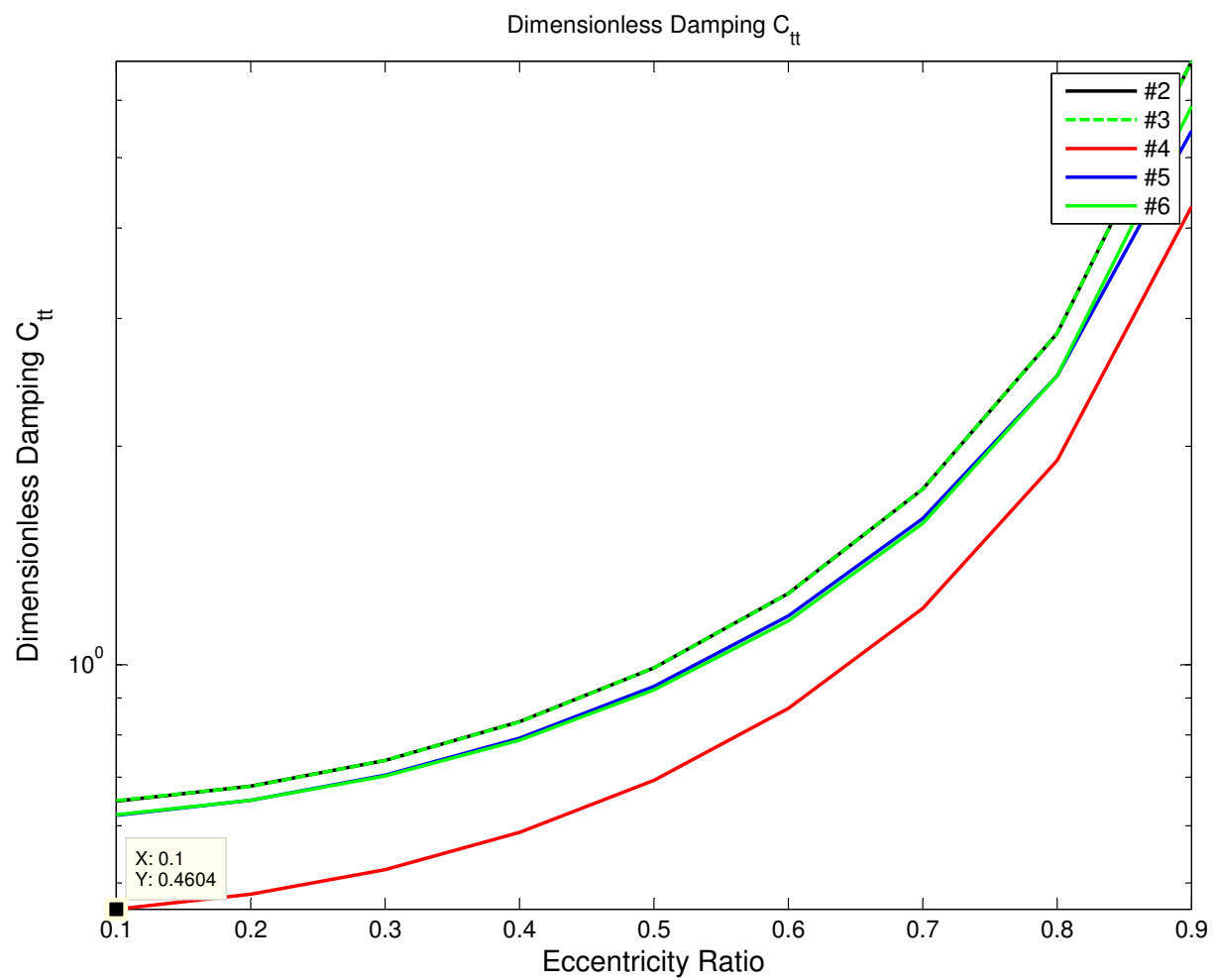

Figure 3.21: $\overline{C_{t t}}$ of Cavitated Film with Hole Number

Figure 3.21 shows the effects of hole numbers on the direct damping. Similar to the cross-coupled damping, four holes generates the minimum damping. The damping of two and three holes are on the top, followed by the cases of five and six holes. Compared to Figure 3.20, the same changes of hole number gives wider variation range for the direct damping than that for the cross-coupled damping, which indicates the hole number has more influence on the direct damping. 


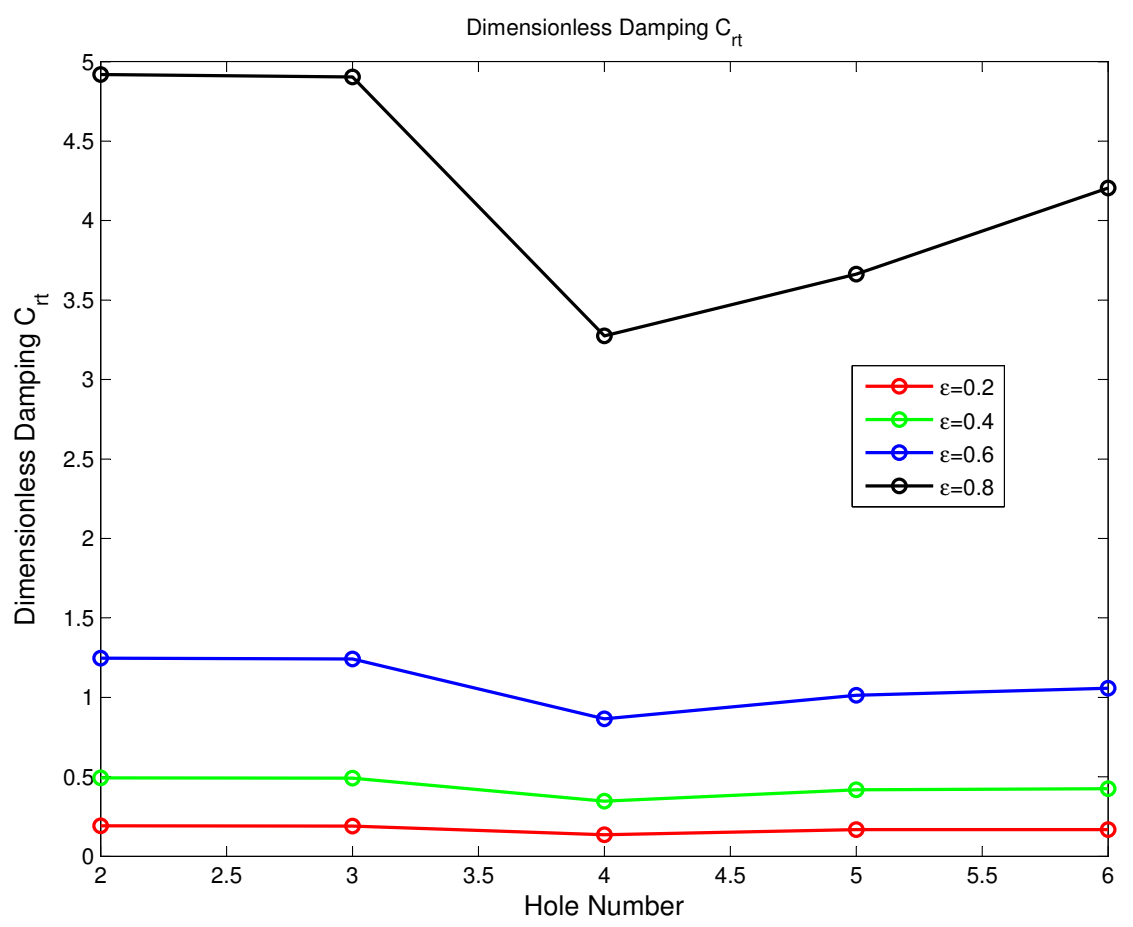

Figure 3.22: $\overline{C_{r t}}$ of Cavitated Film (Fixed Eccentricity)

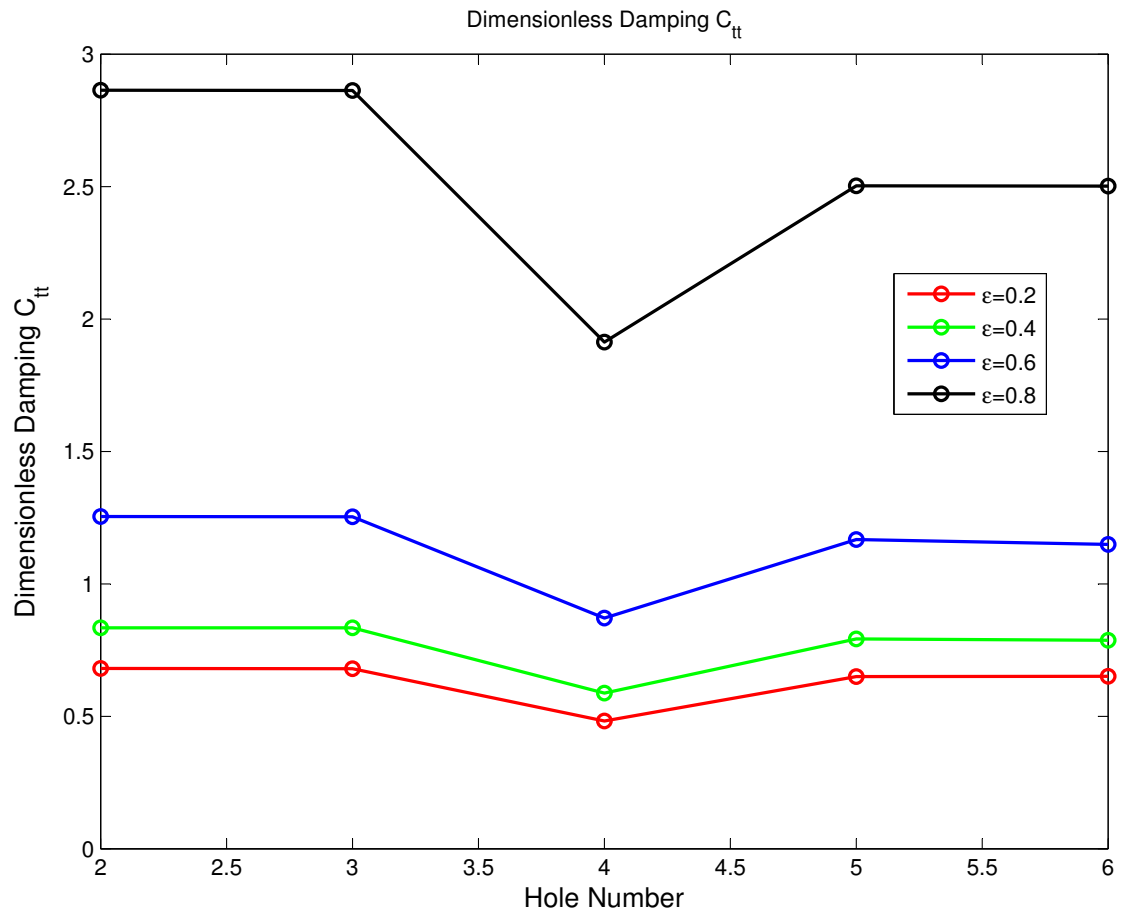

Figure 3.23: $\overline{C_{t t}}$ of Cavitated Film (Fixed Eccentricity) 
Figures 3.22 and 3.23 give the variation of damping with hole numbers for four different

eccentricity cases. Both $\overline{C_{r t}}$ and $\overline{C_{t t}}$ show the change of hole number has less effects on operation with low eccentricity. For $\overline{C_{r t}}$, eccentricity ratio of $20 \%$ does not have obvious variation when subjects to different hole number. This observation indicates that the operation in low eccentricity region resists to the hole number changes. From Figure 3.22 and 3.23, it can also be found that the cross-coupled damping increases more quickly with eccentricity ratio than the direct. Taking the case of two holes for example, the cross-coupled damping starts with $0.19(\epsilon=20 \%)$ and ends at $4.92(\epsilon=80 \%)$, compared to the range from 0.68 to 2.86 for the direct damping. Hence, it is not hard to find that low eccentricity region has more effective damping and generates more stable system.

\subsection{Groove Supply/Discharge}

Groove is another form of oil supply or discharge for squeeze film dampers. Traditional analysis considers the pressure inside the groove as zero, then a closed form pressure distribution is available. However, this simplified model does not agree with the experimental result, which observes non-zero pressure inside the groove [9]. In [7], Rouch found some parts of the pressure within groove is comparable to the pressure in the film land. The current study uses the finite difference method as described before to capture the non-zero pressure inside the groove. A centered groove will be considered. To consider the effects of groove on the pressure distribution, two steps are included. The first step ignores the effects of supply/discharge pressure within the groove. The second one takes into account the supply/discharge condition through holes inside the groove.

\subsubsection{Pressure Distribution}

A damper having the same geometry as in Parts 3.1 and 3.2 is used. Similar to the previous two parts, the axial ends are sealed with piston rings having $C_{p}=0.5, h_{s}=0.5 C$ and ring 
clearance $w_{s}=L / 4$. The groove is centered with axial length of $15 \%$ of entire damper axial length. Using the discretized Reynolds equation 3.7 and applying boundary conditions from piston ring seal, Figure 3.24 shows the damper pressure distribution when the groove depth is 16 times the damper radial clearance. As it shows, the pressure within the groove is not constant as ambient pressure (14.5 psi) but varies with the pressure wave of the film land. The pressure reaches the maximum or minimum as the film land has the largest or smallest pressure, the changing pace in circumferential direction is the same as that of film land. The variation range of groove in circumferential direction is smaller than that of the film land. For any fixed circumferential location, the pressure inside the groove is constant across the axial direction, which is more obvious in Figure 3.25.

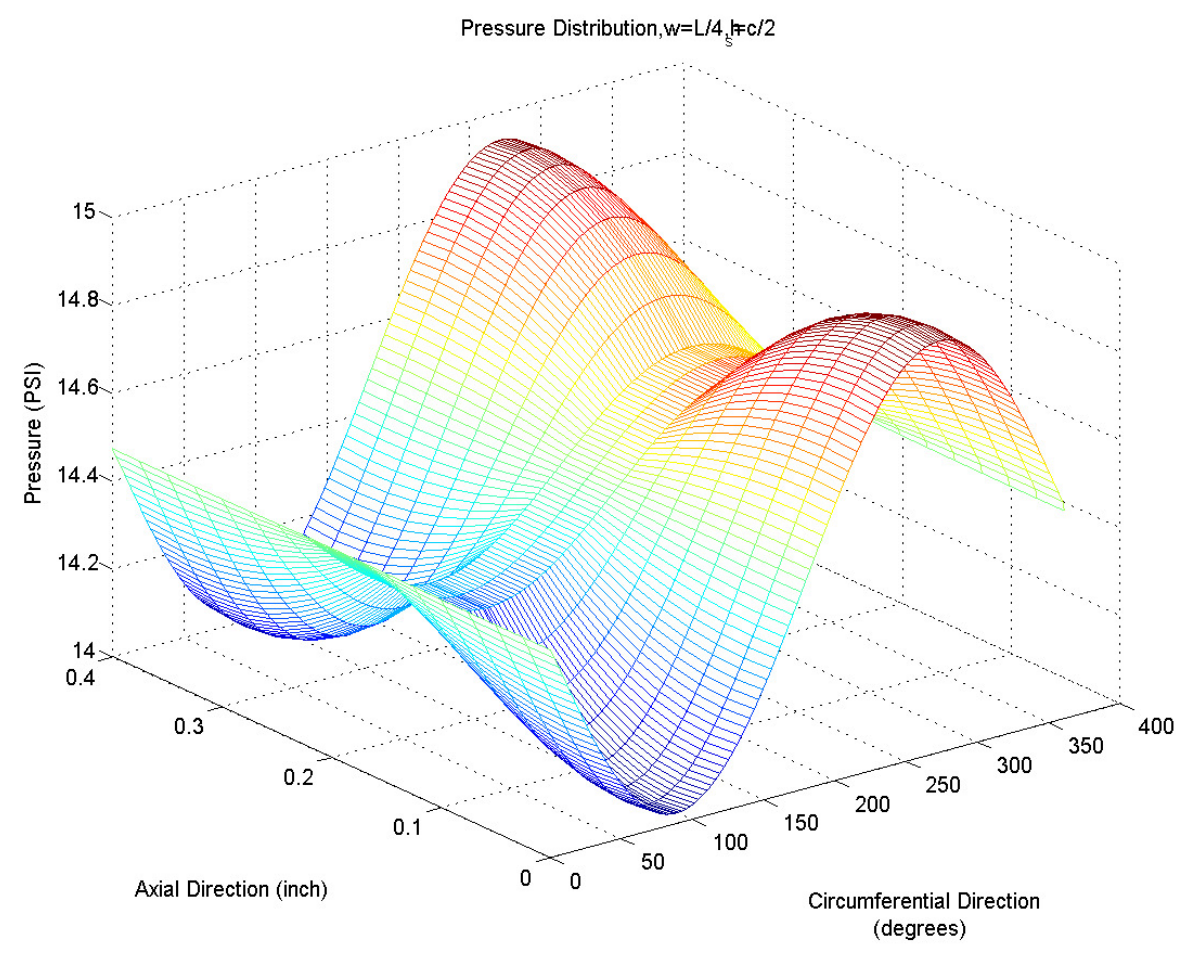

Figure 3.24: Pressure Distribution with Groove Depth of $16 C$

Figure 3.25 shows the axial pressure distribution for the fixed circumferential location where the pressure is maximum. Five different groove depths, varying from $4 C$ to $32 C$, are included. The depth of $4 C$ gives the highest pressure, and the $32 C$ produces the lowest. When the depth is above $16 C$, the pressure distribution does not change very much. The decreasing 
limit, the ambient pressure (14.5 psi), is reached when the groove depth is $32 C$, which means deeper groove can not decrease the pressure further. For all the five cases, the pressure inside the groove is comparable to the pressure generated by the film land. Taking the case of $16 C$ for example, the groove has the pressure of 15.74 psi, compared to the maximum pressure of 15.93 within the film land. This conclusion correlates with other experimental studies [9].

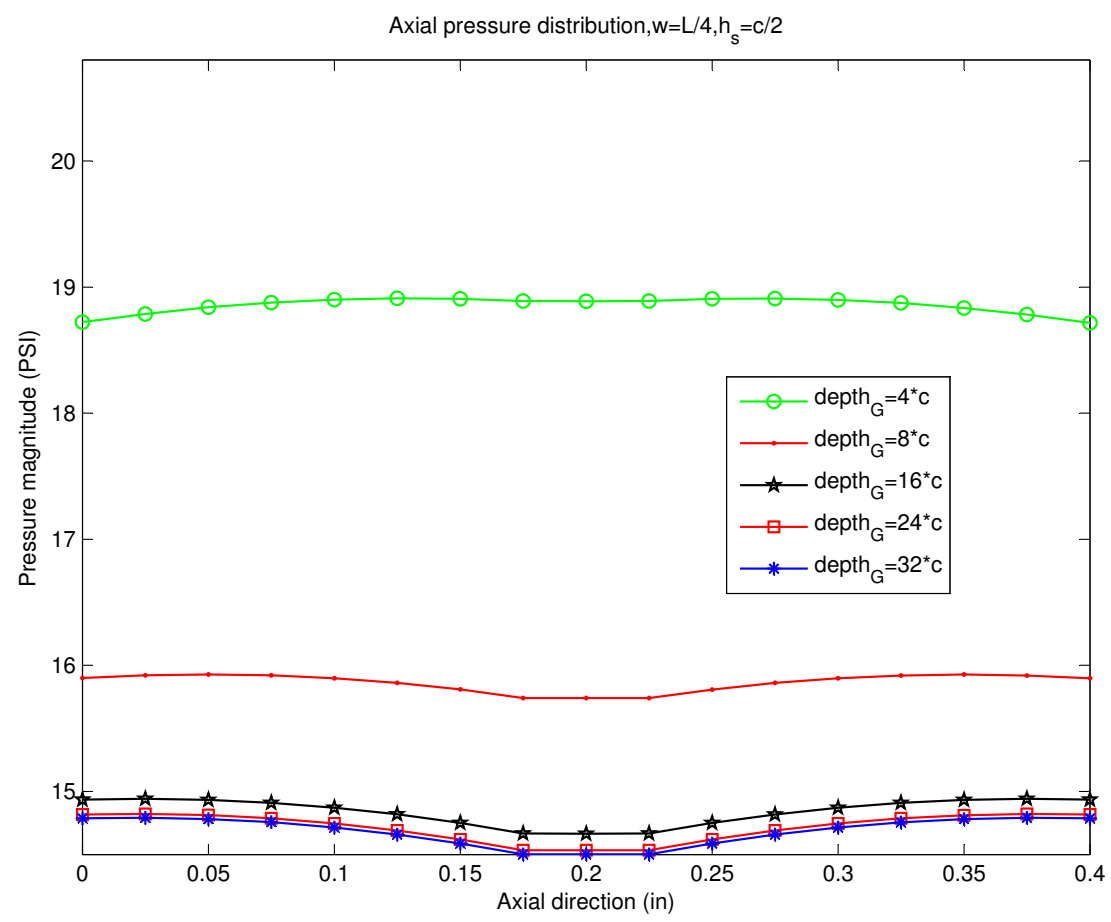

Figure 3.25: Axial Pressure with Varying Groove Depth

Figure 3.25 also indicates the groove depth has dominant effects on the overall pressure distribution. With all other conditions unchanged, the maximum pressure of $4 C$ (18.9 psi) is about $28 \%$ higher than that of $32 C$ (14.76 psi), which tells the smaller groove depth can produce more damping. The groove depth can also affect the shape of axial pressure. From Figure 3.25, the shape of axial distributions are not the similar to each other. The pressure within one side of the film land has parabolic distribution for small groove depth, which generates the largest pressure around the middle part of film land. On the other hand, the large groove depth gives monotonic shape with the film land. The pressure rises from the location of groove and reaches the highest next to the piston ring seal. 
Above analysis does not include the effects of supply/discharge pressure from the central groove. Typical supply/discharge form includes circular holes, which could distribute evenly within the groove in circumferential direction. For the following study, the damper has the same geometry and configurations as the previous one, except two holes are added. The holes are located at $90^{\circ}$ and $270^{\circ}$ in $\theta$ direction and the middle of the groove in axial direction. The one at $90^{\circ}$ is supply channel, and the other is discharge. The pressure for both supply and discharge is 1 atm.

Figure 3.26 depicts the pressure distribution when two holes are presented. Different from Figure 3.24, the pressure inside the groove does not vary with the pressure wave within the film land. Overall, the pressure is quite flat in the circumferential direction, although it has two abrupt changes at the locations of supply and discharge. The pressure shape of the film land does not change much, compared to the distribution without holes. However, its pressure has smaller variation range $(0.6 \mathrm{psi})$ than that of the previous case $(1 \mathrm{psi})$. The damping properties comparison with these two configurations will be studies in part 3.3.2.

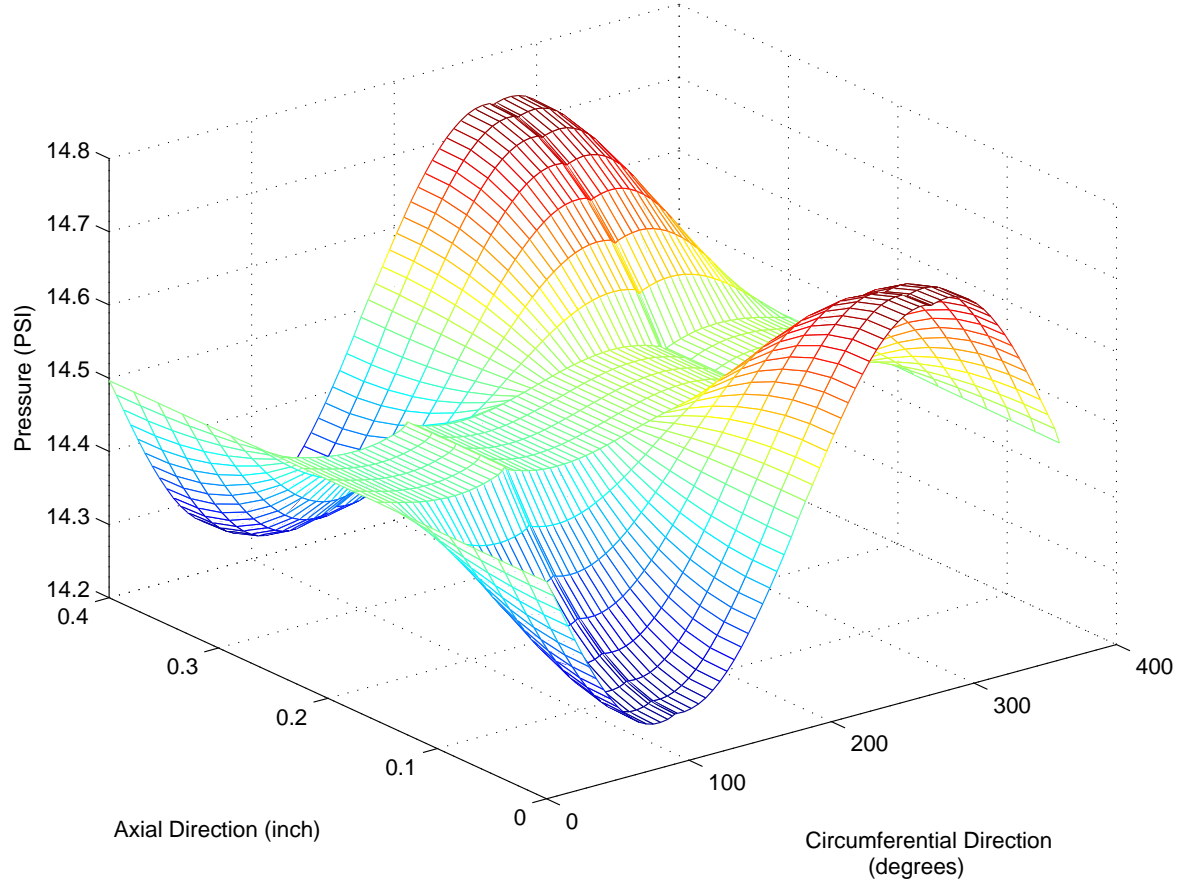

Figure 3.26: Pressure Distribution with Groove Depth of $16 C$ (With Holes) 
As shown by Figure 3.24 and 3.26, the pressure along the piston ring seals has smooth $S$ shape distribution, which is totally different from the case with hole supply/discharge in Figure 3.15. Note that the damper has the same axial length as that in Figure 3.15. It is the presentence of centered groove that eliminates the effects of supply/discharge hole on the pressure within the film land. Therefore, the film outside the groove is not influenced by the holes and has smooth shape of pressure distribution. Caution must be paid to generalize this conclusion. Keep in mind that the groove depth of the current example has the value of $16 C$. With decreasing of the depth, the effects of the groove is diminishing and the effects of hole becomes dominant again. However, it is safe to conclude that the effects of holes on the smoothness of pressure within film land is neglectable for large groove depth; for this damper, it should be larger than $16 C$.

\subsubsection{Force Coefficients of SFDs with Groove}

Same procedures and assumptions in the previous parts are used to investigate the damping capacity of SFDs with central groove. Similarly, the orbit of the journal is assumed to be synchronous and circular centered. The damping coefficients are expressed in dimensionless form within the polar coordinate system.

\section{A Damper with Central Groove - Force Coefficients}

A damper having the same geometry as the previous two sections (3.1.2 and 3.2.2) will be studied in this part. It is sealed by two piston rings which have $C_{p}=0.5, h_{s}=c$ and $w_{s}=L / 4$. The effects with and without supply/discharge holes through the groove will be considered. For the case with holes, two holes locating at $90^{\circ}$ and $270^{\circ}$ in circumferential direction are included. The effects of varying supply pressure of the hole at $90^{\circ}$ and the groove depth are considered as well.

Figures 3.27 and 3.28 depict the damping coefficients of the damper without supply/discharge holes. Both figures consider the cavitation with rupture pressure of 1 atm. 


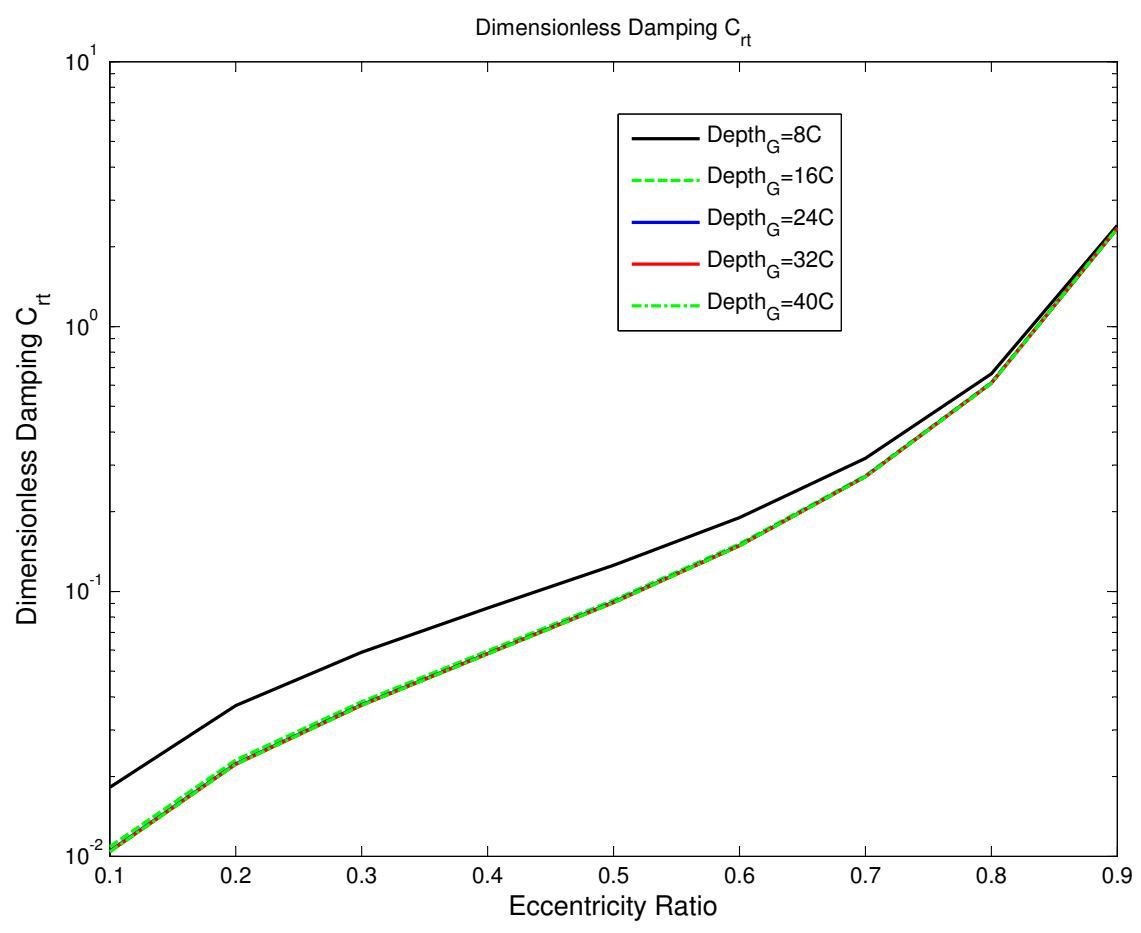

Figure 3.27: $\overline{C_{r t}}$ with Varying Groove Depth(Without Holes)

The effect of central groove depth is included, varying from $8 C$ to $40 C$. In Figure 3.27 , the cross-coupled damping is the maximum with groove depth of $8 C$. The difference between the maximum value and other cases is decreasing with the increase of eccentricity ratio. The operations within high eccentricity (above 80\%) have similar cross-coupled damping. For the low and moderate eccentricity, the damping decreases as the groove becomes deeper. However, the descending trend approaches an asymptotic line, which is evidenced by the overlaps for all the cases with groove depth larger than $16 C$.

Figure 3.28 shows the effect of groove depth on the direct damping. Similar to the cross-coupled damping, it increases monotonically with eccentricity. The difference among the depth cases decreases with the increase of eccentricity ratio. Although shallower groove generates less damping as before, the variation of the depth between $16 C$ and $32 C$ has distinguishable direct damping. The asymptotic groove depth for the direct damping is around $40 C$, larger than $16 C$ for the cross-coupled one. This observation indicates that the SFD has more stable performance as the groove depth decreases within the range from 
$32 C$ to $16 C$. Additionally, both $\overline{C_{r t}}$ and $\overline{C_{t t}}$ do not vary much for groove depth above $40 C$. Therefore, any deeper groove than $40 C$ can not improve the effective damping of the SFD any further.

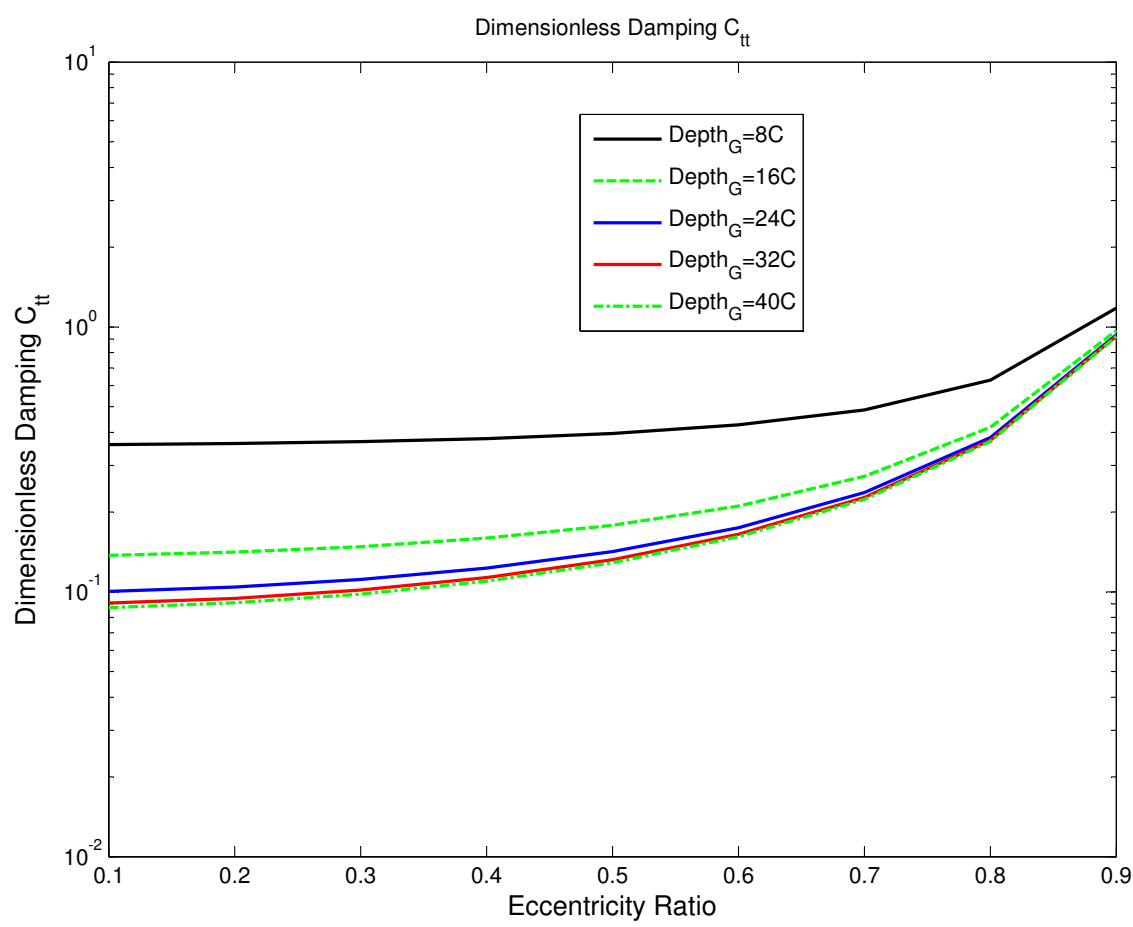

Figure 3.28: $\overline{C_{t t}}$ with Varying Groove Depth(Without Holes)

Figures 3.29 and 3.30 present the damping change with groove depth when supply/discharge holes are considered. Both figures give a similar pattern of the damping variation to the corresponding figure shown previously. The cross-coupled terms for both groove configuration raise with eccentricity faster than the direct terms. Therefore, the effective damping decreases with eccentricity ratio, which is the similar to all the other cavitated dampers. Different from the previous case, the consideration of holes through the central groove generates less damping for both cross-coupled and direct terms. Thus, the effective damping could either increase or decrease depending on the decreasing rate of both direct and cross-coupled damping.

From Figures 3.29 and 3.30, the difference between the maximum damping with the shallowest groove and the minimum with deepest groove is found to be smaller than that of the previous cases without holes. For the cross-coupled damping, the widest variation range 


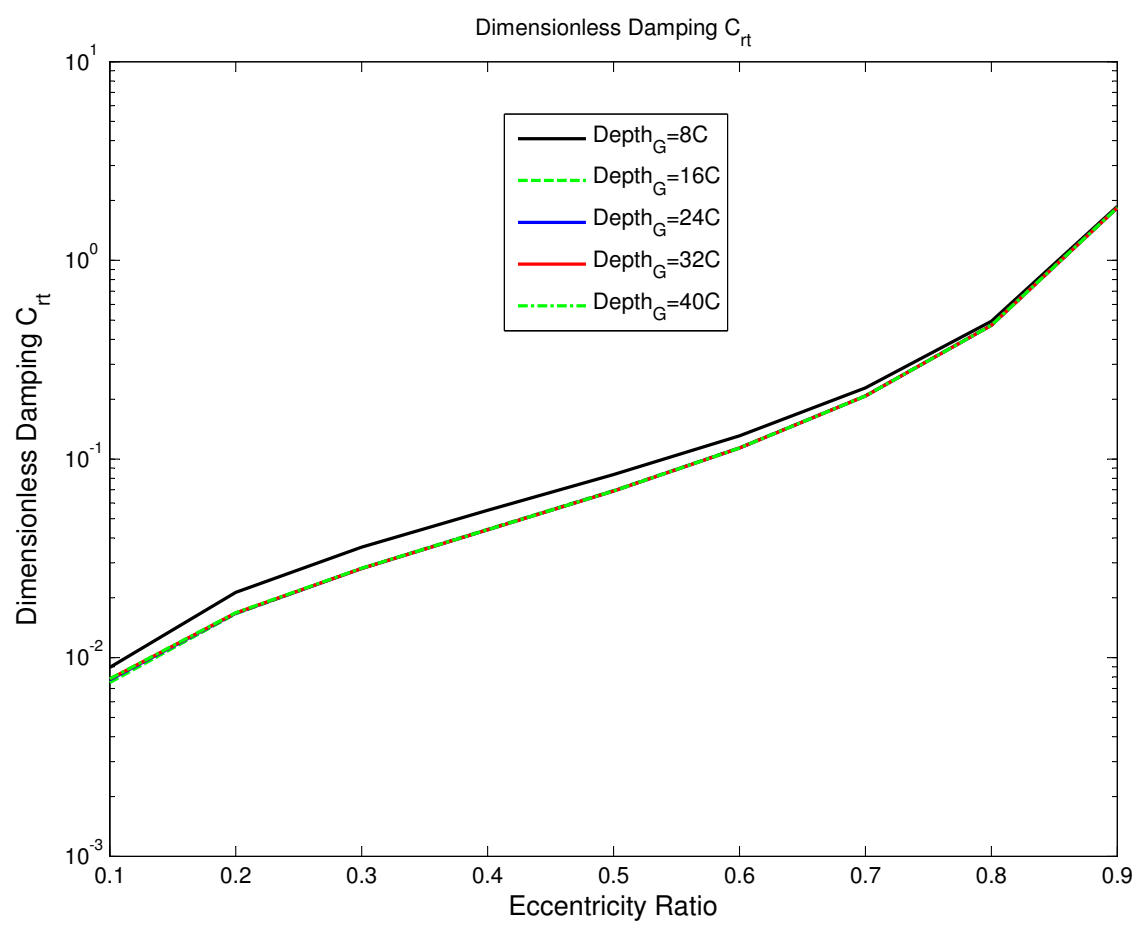

Figure 3.29: $\overline{C_{r t}}$ with Varying Groove Depth(With Holes)

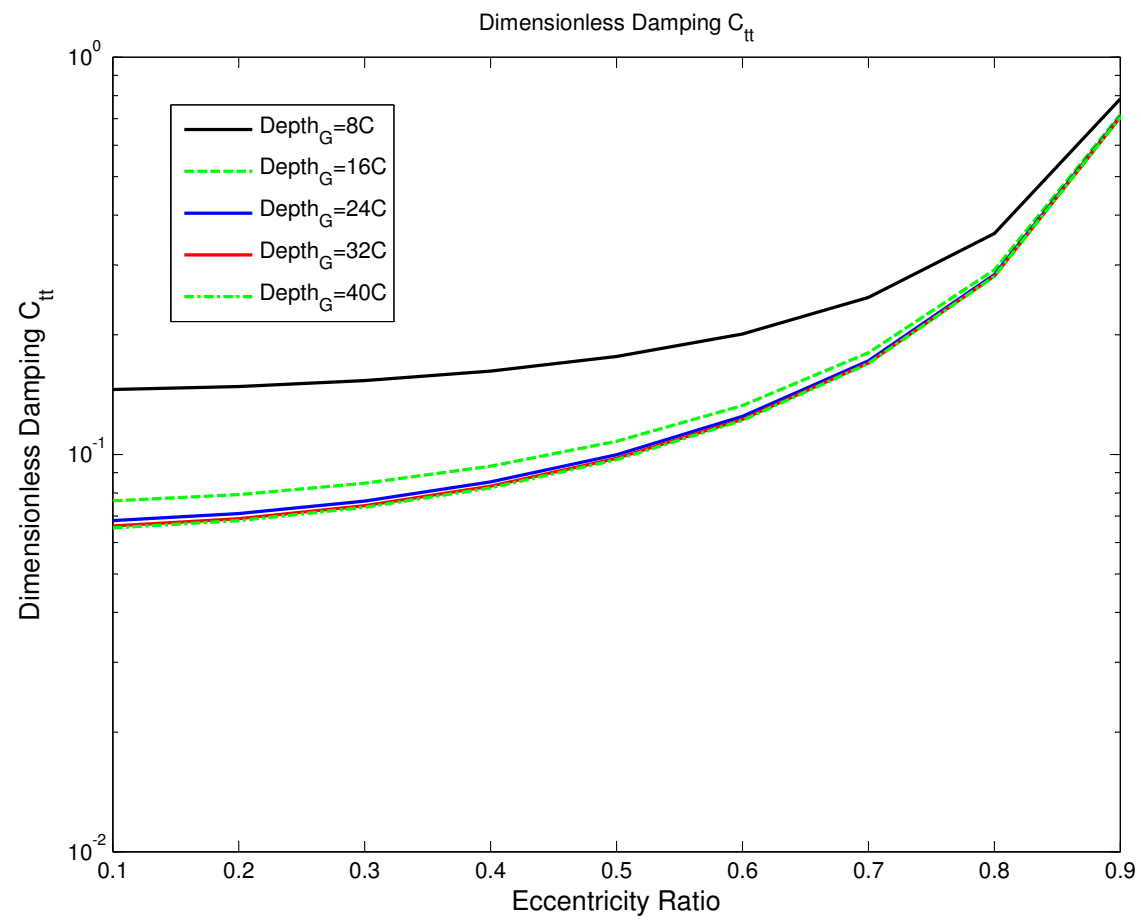

Figure 3.30: $\overline{C_{t t}}$ with Varying Groove Depth(With Holes) 
among different groove depth is 0.015 when the effect of holes is considered, compared to 0.005 for the case with hole. For the direct damping, the maximum range is 0.27 in Figure 3.28, three times as large as that in Figure 3.30. Therefore, the presence of holes inside central groove diminishes the effects of groove depth on the damping generation.

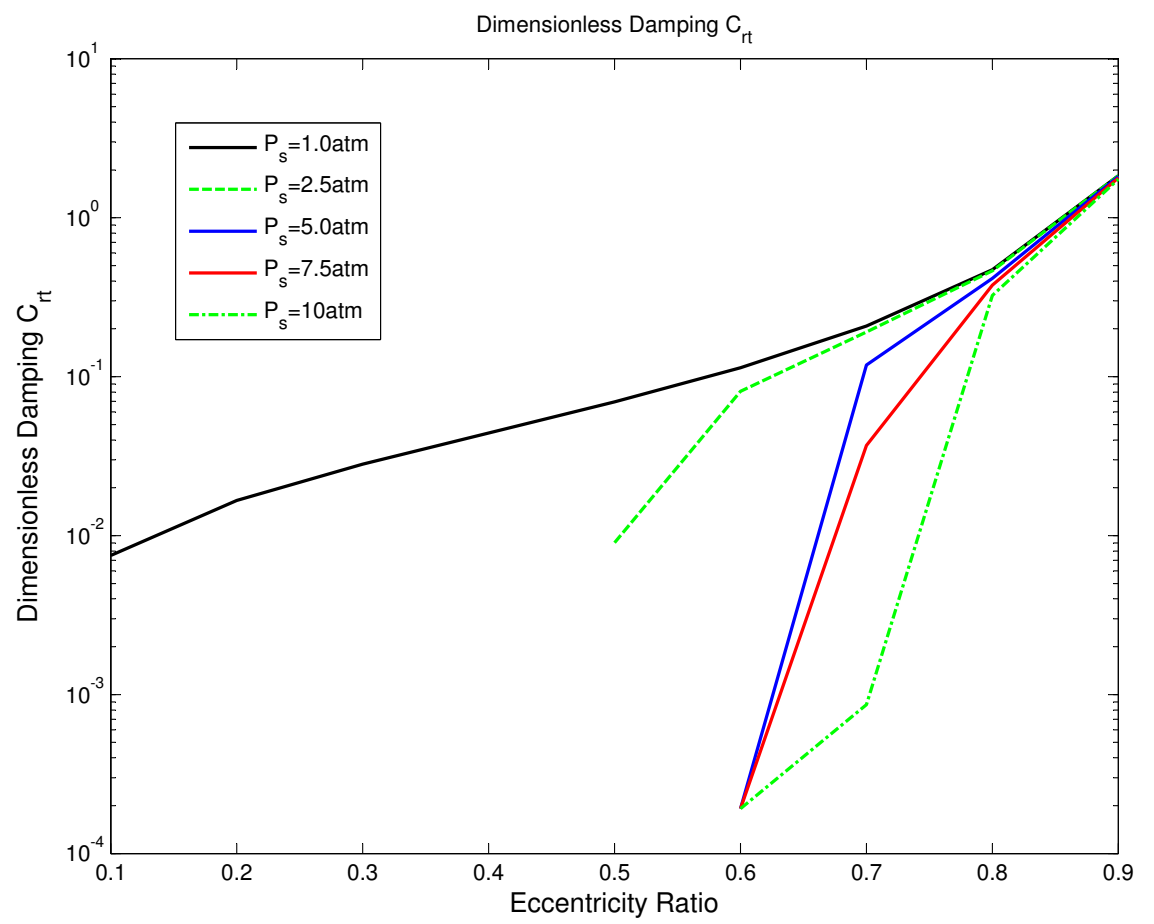

Figure 3.31: $\overline{C_{r t}}$ with Varying Supply Pressure through Holes(Groove)

The effects of supply pressure on dampers with central groove are given in Figure 3.31 and 3.32. The lubricant is supplied through a hole at $90^{\circ}$ to the groove and discharged through the other hole at $270^{\circ}$ in the circumferential direction. Similar to the damper without central groove but only the case of holes supply/discharge in part 3.2.2, the conclusion about the supply pressure in the following can be applied to the study of discharge pressure due to the symmetric configuration of supply and discharge for current damper. The variation range of the supply pressure is from $1 \mathrm{~atm}$ to $10 \mathrm{~atm}$, including five different cases. Except the pressure variation, the groove depth and axial length remain unchanged as $16 \mathrm{C}$ and $0.15 \mathrm{~L}$, respectively. 
Figure 3.31 shows that the supply pressure of 1 atm gives the maximum cross-coupled damping, especially for low and moderate eccentricities. The cross-coupled terms are quite similar to each other for eccentricity ratio above 0.8 . Completely different from the crosscoupled damping subject to groove depth variation, the damping is quite sensitive to the change of supply pressure. Different from the damper with hole supply/discharged only (part 3.2.2), this damper can not generate any cross-coupled damping within low eccentricity range once the supply pressure above $1 \mathrm{~atm}$. For the supply pressure of $2.5 \mathrm{~atm}$, the cross-coupled damping appears only when the eccentricity ratio is larger than 0.5 . For all the other cases with larger supply pressure, the damping presents when the operating range above 0.6 is achieved. This inability of damping generation below high eccentricity region for high supply pressure indicates the effects of supply pressure on the elimination of cavitation. The higher the supply pressure, the less cavitation region inside the film land. When no cavitation happens, the cross-coupled damping becomes zero, which is similar to $2 \pi$-film assumption as given by Gunter et al. [1]. Even for high eccentricity area, the large supply pressure does produce small amount of damping, but the highest pressure remains to generate the smallest value, which indicates the elimination of cavitation by supply pressure as well.

Figure 3.32 gives the direct damping subject to the varying supply pressure. Different from the case without the consideration of holes, the damping with holes inside central groove does not increase monotonically with eccentricity for supply pressure above 1 atm. Contrast to the cross-coupled damping in Figure 3.31, minimum supply pressure (1 atm) produces the minimum direct damping. All the cases with supply larger than 1 atm gives nearly identical damping for the eccentricity range from 0.1 to 0.5 . This means that the direct damping does not vary with supply pressure when no cavitation happens within region having small eccentricity ratio. The pressure only has its effects on direct damping when cavitation takes place. For the range of eccentricity ratio larger than 0.6 , where cavitation happens, the damping increases with supply pressure. This variation is not linear. The supply pressure from 2.5 to 5 atm gives the largest damping enlargement under cavitation condition, while 


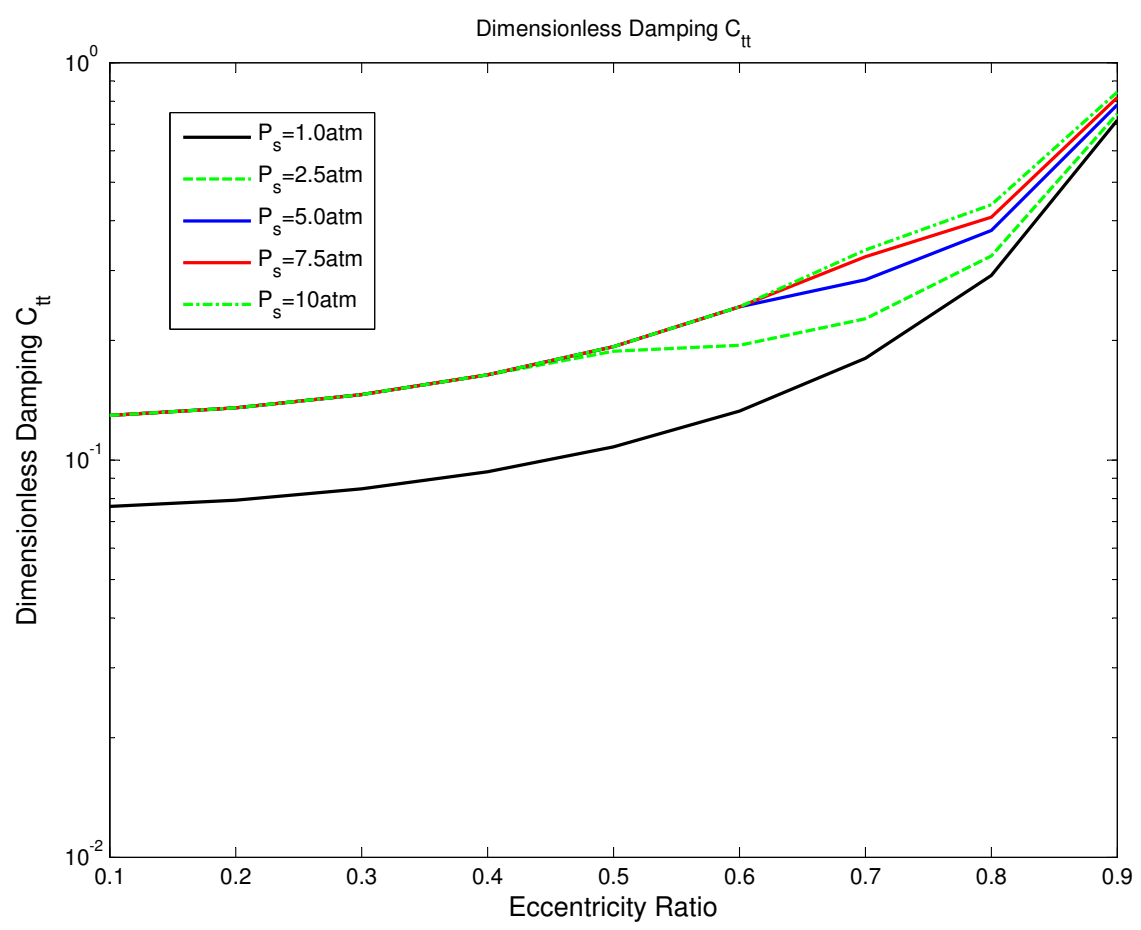

Figure 3.32: $\overline{C_{t t}}$ with Varying Supply Pressure through Holes(Groove)

the same amount change of pressure from 7.5 to $10 \mathrm{~atm}$ has the minimum damping rise. This observation implies that there is a limitation to enlarge the direct damping by increasing the supply pressure.

\subsection{Summary}

This chapter has considered three practical configurations of squeeze film dampers, which include piston ring seals, hole and groove supply/discharge. The finite difference models for both piston ring seals and hole supply/discharge were developed. The pressure distributions of these complicated SFDs were then obtained based on these models. Dimensionless damping coefficients in polar coordinates for each configuration were calculated with the numerical method.

The leakage coefficients and radial seal clearance of piston ring seals were found to have important effects on the damping capacity. For the hole supply/discharge, the impacts of holes 
number and their locations on the damping was studied for the first time. Non-monotonic relation between the hole number and damping coefficients was observed, which highlights the effects of hole number and location on the damping of SFDs.

The developed numerical method gained pressure distribution of SFDs with groove supply/discharge. Non-zero pressure was predicted inside the groove, which correlates with experimental observations. Zero pressure within groove is not guaranteed, except the cases with considerably large groove depth. The increase of supply/discharge pressure through holes into groove was found to eliminate the influence of cavitation and to produce zero cross-coupled damping and constant direct damping within low eccentricity region. 


\section{Chapter 4}

\section{Rotordynamics of Flexible Rotor}

\section{Systems on SFDs using Force}

\section{Coefficients}

With ideal assumptions on the conditions of end seal, supply/discharge and journal orbit, SFDs can be modeled using force coefficients, which are the closed form functions of damper geometries and operating conditions. These assumptions include full or no leakage at the end, zero supply/discharge pressure inside grooves, synchronous Centered Circular Orbit(CCO) of the journal and synchronous Off-centered Circular Orbit(OCO) with small amplitude. Despite the limitations imposed by these assumptions, force coefficients are widely used in simple rotor-bearing systems with SFDs [1, 82, 83, 84]. The main reasons for the popularity of the force coefficient method are its simplicity and computational efficiency compared with nonlinear transient analysis. If a damper satisfies these assumptions, the method can give accurate predictions. Therefore, as long as the analyst is aware of the limitations in the model, this method can be used for rough analysis of a rotor-bearing system with SFDs for example during the initial design stage.

After a brief introduction of the implicit relation between journal dynamics and damper 
reacting forces, this chapter will focus on a Curve Intersection Method to determine the force coefficients for rotordynamics analysis. Three typical assumptions, including full end leakage, no end leakage (to approximate piston ring or O-ring seals), and zero supply/discharge pressure within a central groove, are discussed. The model to incorporate SFDs in a flexible rotor-bearing system for the rotordynamic analysis is included. Both the unbalance responses and stability of the SFDs supported system are documented using the developed method. The rotordynamics with different operating conditions, varying damper parameters, and fluid inertia effects are investigated as well.

\subsection{Force Coefficient Model of SFDs}

The following Reynolds equation governs the pressure inside short-bearing squeeze film dampers. Compared to equation 3.3 shown in part 3.1 of Chapter 3, the following equation 4.1 considers that the pressure gradient in axial direction is much larger than that in the circumferential direction. Therefore, the pressure gradient along the circumferential direction is neglected. This is the base assumption using force coefficients method to model SFDs.

$$
\frac{\partial}{\partial z}\left(\frac{\rho h^{3}}{\mu} \frac{\partial p}{\partial z}\right)=12 \frac{\partial(\rho h)}{\partial t}
$$

Using the same coordinate shown in figure 3.3, the film thickness $h$ can be expressed as,

$$
h=C+e \cos \theta
$$

Therefore, the right side of equation 4.1 equals to,

$$
12 \frac{\partial(\rho h)}{\partial t}=12 \rho(\dot{e} \cos \theta-e \dot{\theta} \sin \theta)
$$

The above equation applies the compressible assumption for the lubricant. Thus, the density 
Chapter 4 | Rotordynamics of Flexible Rotor Systems on SFDs using Force Coefficients68

$\rho$ is time invariant. Now, the pressure is ready to solve by integrating equation 4.1 twice as follows,

$$
P(\theta, z)=6 \frac{\mu}{h^{3}}\left[(\dot{e} \cos \theta-e \dot{\theta} \sin \theta) z^{2}+C_{1} z+C_{2}\right]
$$

with $C_{1}$ and $C_{2}$ are constants to be determined by boundary conditions.

To this point, the forces of SFDs acting on the journal are available by integrating the pressure through film land, which can be expressed in the polar coordinate (defined in Figure 3.3) as,

$$
\begin{aligned}
& F_{r}=\int_{0}^{L} \int_{0}^{2 \pi} P(\theta, z) \cos \theta R d \theta d z \\
& F_{t}=\int_{0}^{L} \int_{0}^{2 \pi} P(\theta, z) \sin \theta R d \theta d z
\end{aligned}
$$

The transformation from polar to fixed coordinate is repeated as follows, shown by equation 3.13 in chapter 3 .

$$
\left[\begin{array}{l}
F_{x} \\
F_{y}
\end{array}\right]=\left[\begin{array}{cc}
\sin \varphi & \cos \varphi \\
-\cos \varphi & \sin \varphi
\end{array}\right]\left[\begin{array}{c}
F_{r} \\
F_{t}
\end{array}\right]
$$

Equations from 4.4 to 4.6 show that the forces generated by squeeze film dampers are dependent on both the location and velocity of journal. On the other hand, for any given rotor-bearing system with SFDs, the journal location and velocity are determined by forces from SFDs, imbalance on the rotor and external loads et cetera. These kinds of relation between journal dynamic behaviors and SFD forces is implicit. The following parts will show more details to reveal this relation. 


\subsubsection{Open End(Full Leakage)}

For the assumption with open ends, the pressure distribution can be determined using the boundary conditions at ends,

$$
P(\theta, 0)=P(\theta, L)=P_{\text {ambient }}
$$

$P_{\text {ambient }}$ is the ambient pressure. To simplify even further, zero pressure can be assumed for above equation [1], which gives,

$$
P(\theta, 0)=P(\theta, L)=0
$$

Apply the above conditions to equation 4.4, the two constants can be obtained as,

$$
\begin{aligned}
& C_{1}=-(\dot{e} \cos \theta-e \dot{\theta} \sin \theta) L \\
& C_{2}=0
\end{aligned}
$$

Thus, the pressure for the open end SFDs is

$$
P(\theta, z)=\frac{6 \mu}{h^{3}}(\dot{e} \cos \theta-e \dot{\theta} \sin \theta)\left(z^{2}-L z\right)
$$

Substituting above pressure into equation 4.5 and assuming Centered Circular Orbit(CCO) result in,

$$
\begin{aligned}
& F_{r}=-\frac{\mu L^{3} R C \varepsilon \dot{\varphi}}{C^{3}} \int_{0}^{2 \pi} \frac{\sin \theta}{(1+\varepsilon \cos \theta)^{3}} \cos \theta d \theta \\
& F_{t}=-\frac{\mu L^{3} R C \varepsilon \dot{\varphi}}{C^{3}} \int_{0}^{2 \pi} \frac{\sin \theta}{(1+\varepsilon \cos \theta)^{3}} \sin \theta d \theta
\end{aligned}
$$

$\dot{\varphi}$ is angular speed of precession. It can be found that the integrand for radial force $F_{r}$ is an odd function, which indicates $F_{r}$ is zero if all the pressure in circumferential direction from 0

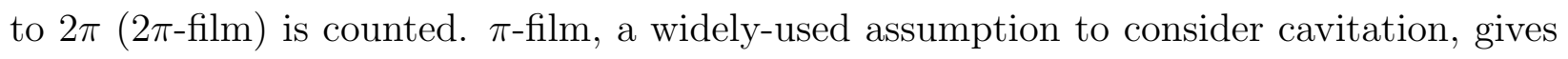


non-zero radial forces. Equations 4.12 and 4.13 give the SFD forces for $\pi$-film and $2 \pi$-film, respectively. The tangential force of $2 \pi$-film is twice that of the $\pi$-film.

$\pi$-film,

$$
\begin{aligned}
& F_{r}=-\frac{2 \mu L^{3} R \varepsilon(e \dot{\varphi})}{C^{3}\left(1-\varepsilon^{2}\right)^{2}} \\
& F_{t}=-\frac{\mu L^{3} R \pi(e \dot{\varphi})}{2 C^{3}\left(1-\varepsilon^{2}\right)^{1.5}}
\end{aligned}
$$

$2 \pi$-film

$$
\begin{aligned}
& F_{r}=0 \\
& F_{t}=-\frac{\mu L^{3} R \pi(e \dot{\varphi})}{C^{3}\left(1-\varepsilon^{2}\right)^{1.5}}
\end{aligned}
$$

Note that the term of $e \dot{\varphi}$ in the previous two equations is the precession velocity $V_{t}$ in tangential direction. Expressing the SFD forces in terms of $V_{t}$ as done in part 3.1.2, the cross-coupled and direct damping are readily available. Table 4.1 gives the damping for both $\pi$-film and $2 \pi$-film.

$$
\begin{aligned}
& F_{r}=C_{r t} V_{t} \\
& F_{t}=C_{t t} V_{t}
\end{aligned}
$$

Table 4.1: Damping Coefficients of Open-end SFDs under CCO

\begin{tabular}{c|c|c}
\hline Cavitation Condition & $C_{r t}$ & $C_{t t}$ \\
\hline$\pi$-film (Cavitated) & $\frac{2 \mu L^{3} R \varepsilon}{C^{3}\left(1-\varepsilon^{2}\right)^{2}}$ & $\frac{\mu L^{3} R \pi}{2 C^{3}\left(1-\varepsilon^{2}\right)^{1.5}}$ \\
\hline $2 \pi$-film (Uncavitated) & 0 & $\frac{\mu L^{3} R \pi}{C^{3}\left(1-\varepsilon^{2}\right)^{1.5}}$ \\
\hline
\end{tabular}

The implicit relation between damper forces and journal dynamics can also be told by the damping coefficients in above table, since both $C_{r t}$ and $C_{t t}$ are the functions of an unknown, the eccentricity ratio $\varepsilon$. The $2 \pi$-film gives one time more direct damping than that of $\pi$-film, and its cross-coupled damping is zero. Therefore, the effective damping of $2 \pi$-film is much larger than that of $\pi$-film.

\subsubsection{Central Groove Supply/Discharge with Full or No Leakage}

The main assumption for central groove supply/discharge in the force coefficients model is zero pressure through the entire groove, although it is not always the case for shallow grooves, 
as observed in part 3.3. Gunter et al. [1] applied this assumption and approximated the damping coefficients for grooved dampers with full and no leakage.

For the case of central groove with full leakage, it can be considered as two identical dampers separated by the groove and having half of the original axial length, as shown in Figure 4.1. For each film land, the damping can be obtained using the expressions in table 4.1 and replacing the axial length with half the original, which means the total forces and damping of one film land reduces to one eighth of original value. Thus, the total damping of a central grooved damper with full leakage is one fourth of the one without groove.

For central grooved dampers with end seals, as shown in Figure 4.2, the pressure disappears at the location of groove. Based on this observation, Gunter et al. [1] assumed the pressure profile of sealed damper with central groove is equivalent to the open-end damper. This assumption leads to the conclusion that the damping capacities in figure 4.2 are the same as those listed in table 4.1.

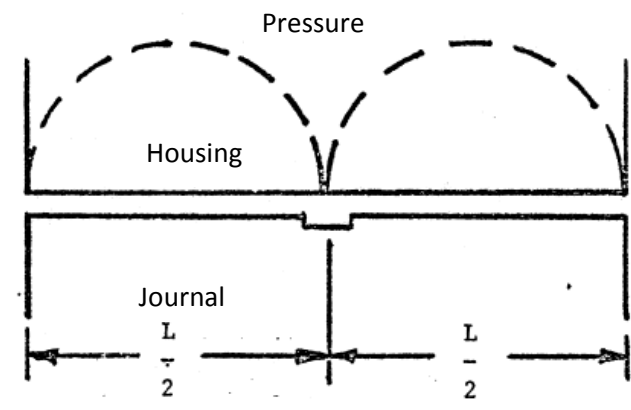

Figure 4.1: Full Leakage [1]

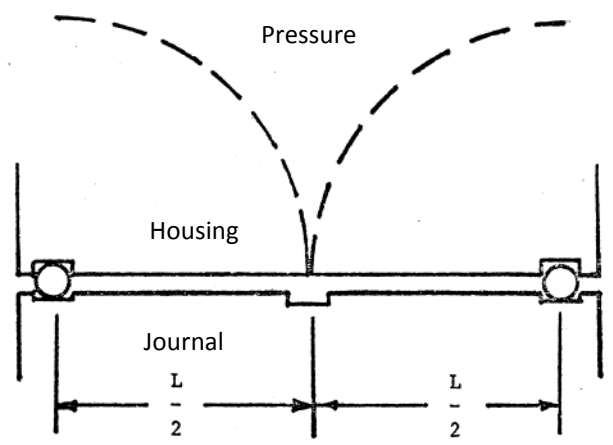

Figure 4.2: No Leakage [1]

\subsubsection{Open End SFDs with Inertia Effects}

San Andrés [5] developed force coefficients for short SFDs to consider the fluid inertia effect. To include this effect, the damper forces were expressed in the polar coordinate as follows, 
Chapter 4 | Rotordynamics of Flexible Rotor Systems on SFDs using Force Coefficients72

$$
\begin{aligned}
& F_{r}=\int_{0}^{L} \int_{0}^{2 \pi} P(\theta, z) \cos \theta R d \theta d z=C_{r t} V_{t}+M_{r r} a_{r} \\
& F_{t}=\int_{0}^{L} \int_{0}^{2 \pi} P(\theta, z) \sin \theta R d \theta d z=C_{t t} V_{t}+M_{t r} a_{r}
\end{aligned}
$$

Different from Equation 4.5, the pressure in above equation is calculated using NavierStokes equation. To obtain analytical solutions with the inertia effect, the synchronous CCO precession with open-end assumption was applied, same as the previous two sections. The fluid inertia of the lubricant under different Reynolds number were discussed. Table 4.2 gives the mass and damping coefficients including inertia effect.

Table 4.2: Force Coefficients of Open-end SFDs W/ inertia [5]

\begin{tabular}{c|c}
\hline & $2 \pi$-film solution (No cavitation) \\
\hline$C_{r t}$ & 0 \\
\hline$C_{t t}$ & $\frac{\mu L^{3} R \pi}{C^{3}\left(1-\varepsilon^{2}\right)^{1.5}}$ \\
\hline$M_{t r}$ & 0 \\
\hline$M_{r r}$ & $\frac{\pi \mu L^{3} R}{6 C^{3} \varepsilon^{2} \omega} \frac{1-\gamma}{\gamma} R e\left[v_{1}+v_{2}(\gamma-1)\right]$ \\
\hline
\end{tabular}

Note that only $2 \pi$-film is considered for the analytical solutions. If $\pi$-film is considered, numerical method is needed to count the inertia effect on the cavitated film [5].

In Table $4.2, \gamma=\left(1-\varepsilon^{2}\right)^{0.5}$. Re is the Reynolds number for squeeze film dampers. $v_{1}$ and $v_{2}$ are coefficients dependent on the value of $R e$ for a given damper. Table 4.3 lists their value for different Reynolds numbers [5].

Table 4.3: $v_{1}$ and $v_{2}$ with different $R e$

\begin{tabular}{c|c|c}
\hline$R e$ & $v_{1}$ & $v_{2}$ \\
\hline$\ll 1$ & 1.2 & $102 / 35$ \\
\hline moderate & 1.0 & $84 / 35$ \\
\hline$\gg 1$ & 1.0 & $70 / 35$ \\
\hline
\end{tabular}

\subsection{Curve Intersection Method}

As shown in Part 4.1, the forces and damping of SFDs are functions of journal eccentricities for cases with and without fluid inertia effects. Given a rotor-bearing system on SFDs, the 
eccentricity is dependent not only on the damper load capacities but also on external loads. Therefore, it is not known beforehand. To handle this problem, traditional methods choose some iteration procedures to calculate the journal eccentricity for steady responses. Greenhill and Nelson [64] used an iteration procedure to determine the damping coefficients of SFDs subject to airfoil imbalance. The SFD studied is short bearing with open-end and $\pi$-film assumptions. Fluid inertia effect is not included. More recently, Ertas et al. [85] applied a similar iteration method to study the synchronous response of a rotor on SFDs with the same assumptions as in [64]. Figure 4.3 shows the typical iteration scheme for these procedures.

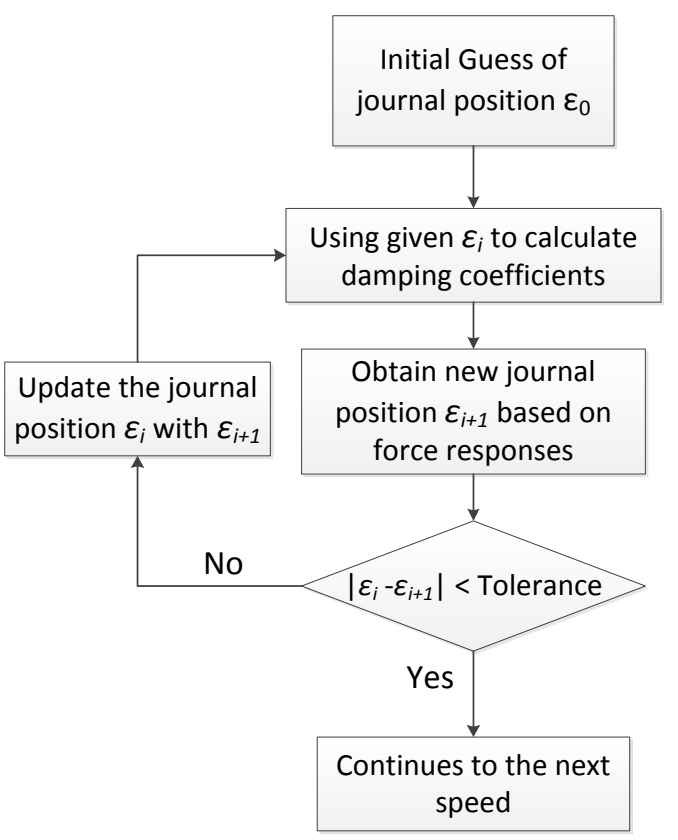

Figure 4.3: Typical Iteration Scheme

The main problem with the traditional iteration method is that it is not robust to obtain the steady state solutions of journal eccentricity. The calculation speed and accuracy are highly dependent on the initial guess of the solution at the beginning. If the distance between the initial guess and the final solution is too large, the iteration method could be unconverged. It is true that, if the initial guess is close to the the solution, the converged solution might be obtained quickly. The problem for the latter case is how to guarantee the initial guess is 
Chapter 4 | Rotordynamics of Flexible Rotor Systems on SFDs using Force Coefficients74

close enough to the solution. Unless for some familiar rotor-bearing systems to the user, it is doubtful to have an ideal initial guess every time.

Another problem with the traditional iteration method is the sensitivity. It is sensitive not only to the convergence tolerance (shown in figure 4.3) but also to the loads of the rotating system. Greenhill and Nelson [64] found their iteration procedure can not converge if large unbalance forces are present. The reason behind this observation is that, the SFDs behave more nonlinearly as higher unbalance forces act on the rotor, and the traditional method is unable to handle highly nonlinear performances successfully.

To solve the problems with traditional iteration methods, a Curve Intersection Method(CIM) is developed in this part. This method is based on the relation between the input of journal position (the eccentricity) and the output responses with the damping coefficients under the input condition. The range of journal eccentricity ratio $\varepsilon$ for the input can be any value in $[0,1)$. For each point within the range, the corresponding response of the journal position can be calculated. The connection of these outputs forms a performance curve of a given damper. Curve A in figure 4.4 shows an example of performance curve of a target damper.

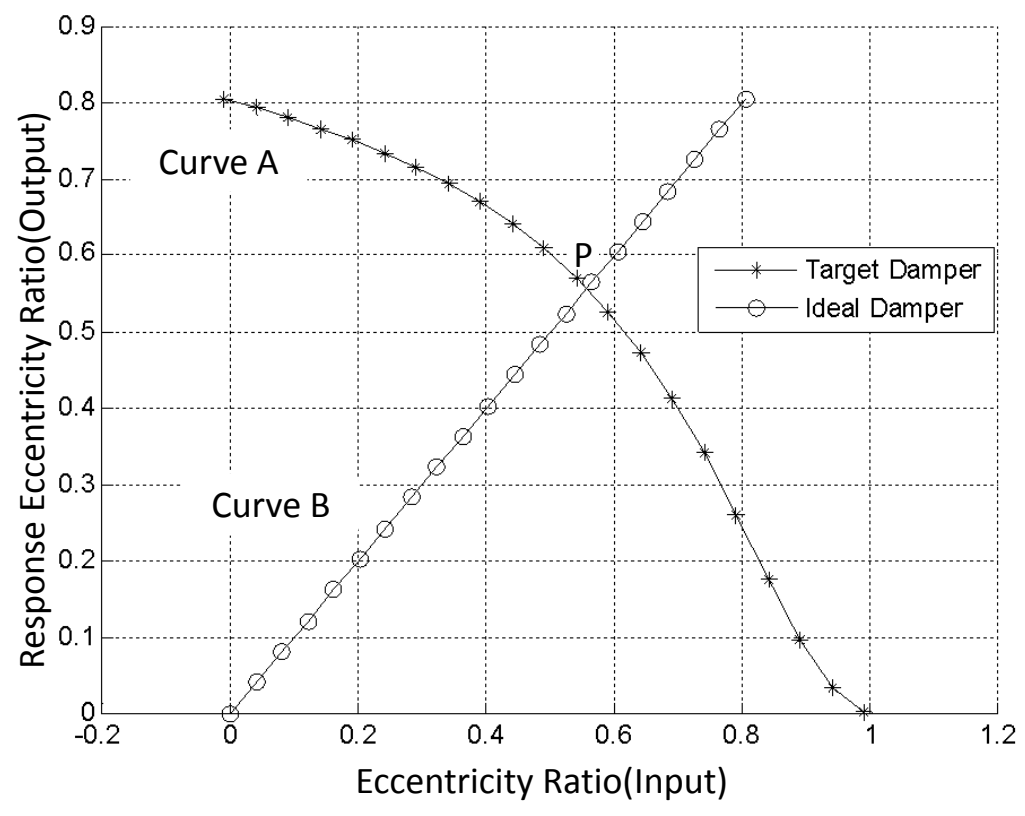

Figure 4.4: Illustration of Curve Intersection Method

In the Curve Intersection Method, another ideal damper is introduced, which generates 
the same response eccentricities as the input. Hence, the performance curve of the ideal damper is a straight line with slope of one, as curve B shown in Figure 4.4. The combination of the two curves results in intersection point(s) and gives the steady state solution(s) of the target damper. In the figure, the corresponding eccentricity ratio of intersection point $P$ is the solution for the damper.

Compared with traditional iteration procedure, the developed CIM method is more robust to the performance variations of SFDs. Linear or highly nonlinear behavior can be obtained by the performance curve in the CIM. The shape and smoothness of the curve reflect the nonlinear extent of the damper. No matter how much nonlinearity is present, there is always a performance curve and the solution can be obtained quickly through intersection. The robustness of the CIM method can also be shown by its ability to find multiple solutions(intersections) and to determine if the target damper does not have enough load capacity for external forces. The multiple intersections might be possible if a damper is subject to excessive unbalance forces, which can produce jump responses [86]. For the damper with low load capacity, the CIM method can indicate this situation by the event of no intersection between the two curves. It is difficult for the traditional iteration method to handle the cases with multiple solutions and low load capacity. The following two sections will apply the curve intersection method for both unbalance responses and stability analysis for a rotor bearing system with a squeeze film damper.

\subsection{Incorporate SFDs Using Force Coefficients}

To incorporate SFDs for rotordynamics analyses, the rotors are modeled using the finite element method. This method is one of the classical methods for rotordynamics analysis. It has been discussed extensively since 1972, when Ruhl and Booker [87] first developed the method for rotating systems. Within this method, the rotor shaft is discretized into beam elements having mass matrix, stiffness matrix and damping matrix(if any). The disks 
Chapter 4 | Rotordynamics of Flexible Rotor Systems on SFDs using Force Coefficients76

and blades are approximated with lumped mass, polar and transverse inertia. The bearings might be considered by either KCM (stiffness, damping and mass) model or KC (stiffness and damping) model [88]. The model of whole rotor-bearing system can be established by assembling the element matrices of mass, stiffness and damping of the entire system. Since lots of related works have been done in the past [89, 90, 91, 92, 93], details about shaft mass, stiffness and damping calculation and element matrices assembling will not be discussed here. Only the consideration and incorporation of SFDs for dynamic analysis is included.

Figure 4.5 gives a spring-damping-mass model of a squeeze film damper with journal (includes bearings in series). Both SFDs and in-series bearings are modeled with stiffness and damping coefficients. Note that the stiffness of the SFD here is an effective stiffness, which comes from the cross-coupled damping property as the journal precesses inside. The cross-coupled and direct damping of the SFD will be determined by the Curve Intersection Method developed in part 4.2. The mass term in the figure includes the mass of the precessing journal, which contains the mass of in-series bearings and surrounding arrangements. For applications of bearings mounted directly to the ground, this kind of mass does not contribute to the dynamics analysis. However, the mass needs to be considered for the applications in series with SFDs due to the precession motion. The mass term in Figure 4.5 might also include the effective mass of the squeeze film if the effects of fluid inertia are considered. Figure 4.5 only shows the model of one Degree-Of-Freedom(DOF). The complete model considers two DOF for the damper, translational motions in both $x$ and $y$ direction.

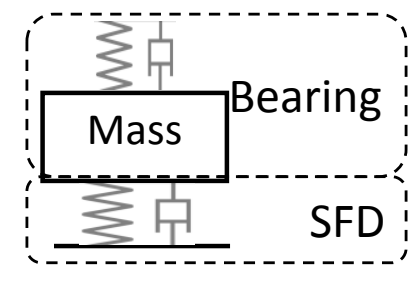

Figure 4.5: Spring-Damping-Mass Model of SFD with Journal

Figure 4.6 depicts an example of a finite element beam model of a rotor-bearing system on squeeze film dampers. There is one SFD in series with the ball bearing (Brg2) at the right 


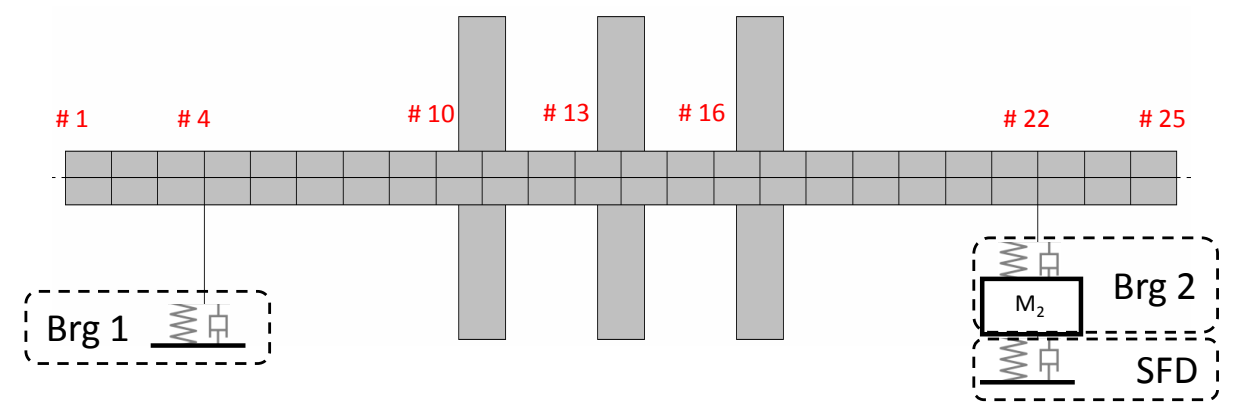

Figure 4.6: Finite Element Model of a Three Mass Rotor with SFD

end. The shaft is discretized into 24 elements(25 nodes), with three identical disks locating at node 10, 13 and 16, respectively. The left ball bearing connects to node 4 and the right one to node 22. To incorporate the damper, its stiffness and damping matrix connect to node 22 , and the diagonal mass matrix is added to that of the shaft. The shaft has total DOF of 100, considering four DOF for each node (two translational and two rotational). The damper adds another two to the whole system. Hence, the global matrices (including mass, stiffness and damping) after assembling has the size of $102 \times 102$. More details about how to assemble the element matrices can be found in finite element books such as [94].

After assembling all the element matrices, the equation of motion for the rotor-bearing system can be obtained as follows,

$$
[\mathbf{M}]\{\ddot{\mathbf{x}}\}+([\mathbf{C}]+\Omega[\mathbf{G}])\{\dot{\mathbf{x}}\}+[\mathbf{K}]\{\mathbf{x}\}=\{\mathbf{f}\}
$$

Equation 4.16 establishes the base for both unbalance and stability analysis. More details about these two analyses are discussed in the following parts.

\subsection{Unbalance Responses Using Force Coefficients}

This section focuses on the unbalance response analysis of rotor-bearing systems on SFDs. The effects of rotor operating conditions (including unbalance magnitude and location, rotating speed) and damper configurations (including $L / D$ and $C / R$ ) are studied. The responses 
Chapter 4 | Rotordynamics of Flexible Rotor Systems on SFDs using Force Coefficients78 with and without fluid inertia effects are compared as well. Before the detailed discussion of response results, the mathematical model for the simulations of unbalance responses is required.

\subsubsection{Mathematical Model for Unbalance Responses with SFDs}

To be compatible with the stability analysis, the equation of motion 4.16 is converted to state space form as,

$$
\left[\begin{array}{cc}
\mathbf{M} & \mathbf{0} \\
\mathbf{0} & \mathbf{M}
\end{array}\right]\{\dot{\mathbf{u}}\}+\left[\begin{array}{cc}
\mathbf{0} & -\mathbf{M} \\
\mathbf{K} & \overline{\mathbf{C}}
\end{array}\right]\{\mathbf{u}\}=\left[\begin{array}{l}
\mathbf{0} \\
\mathbf{f}
\end{array}\right]
$$

where, $\overline{\mathbf{C}}=\mathbf{C}+\Omega \mathbf{G} ; \mathbf{u}$ is a state space vector, equals to $\left[\begin{array}{c}\mathbf{x} \\ \dot{\mathbf{x}}\end{array}\right]$

For current rotor-bearing system subject to unbalance excitation only, the vector $u$ can be expressed as,

$$
\{\mathbf{u}\}=\{\mathbf{U}\} e^{j \Omega t}
$$

The unbalance force $f$ is,

$$
\{\mathbf{f}\}=\{\mathbf{F}\} e^{j \Omega t}
$$

Substituting above two equations into equation 4.17 gives,

$$
\Omega\left[\begin{array}{cc}
\mathbf{M} & \mathbf{0} \\
\mathbf{0} & \mathbf{M}
\end{array}\right]\{\mathbf{U}\}+\left[\begin{array}{cc}
\mathbf{0} & -\mathbf{M} \\
\mathbf{K} & \overline{\mathbf{C}}
\end{array}\right]\{\mathbf{U}\}=\left[\begin{array}{l}
0 \\
\mathbf{F}
\end{array}\right]
$$

Further simplification of above equation results,

$$
\left[\Omega \mathbf{I}+\mathbf{A}^{-1} \mathbf{B}\right]\{\mathbf{U}\}=\left[\begin{array}{c}
0 \\
\mathbf{M}^{-1} \mathbf{F}
\end{array}\right]
$$

where, 


$$
\mathbf{A}=\left[\begin{array}{cc}
\mathbf{M} & \mathbf{0} \\
\mathbf{0} & \mathbf{M}
\end{array}\right], \mathbf{B}=\left[\begin{array}{cc}
\mathbf{0} & -\mathbf{M} \\
\mathbf{K} & \overline{\mathbf{C}}
\end{array}\right]
$$

From equation 4.21 , the response vector $\{\mathbf{U}\}$ is solved as,

$$
\{\mathbf{U}\}=\left[\Omega \mathbf{I}+\mathbf{A}^{-1} \mathbf{B}\right]^{-1}\left[\begin{array}{c}
\mathbf{0} \\
\mathbf{M}^{-1} \mathbf{F}
\end{array}\right]
$$

The above derivations are valid for synchronous responses of any rotor-bearing system subject to unbalance forces, including these with SFDs. For systems with SFDs, the $\mathbf{K}$ and C matrices are obtained after the steady state damper eccentricity is determined using Curve Intersection Method. Equation 4.22 is used for every point on the performance curve shown in Figure 4.4. Figure 4.7 depicts the scheme of the unbalance response analysis using the Curve Intersection Method.

\subsubsection{Responses with Varying Operating Conditions of the Rotor}

The example to demonstrate the method developed in previous parts is the one shown in Figure 4.6. The geometries of the rotor are listed in table 4.4. The operating speed range is from 1,000 to 10,000 rpm. The length of shaft element is one inch. The polar and transverse inertia of the disk are 36 and $18.67 \mathrm{lb} \cdot \mathrm{in}^{2}$, respectively. The ball bearing has stiffness of $600,000 \mathrm{lbf} / \mathrm{in}$ and mass of $1.6 \mathrm{lbm}$. The damping of the ball bearing is small enough to be neglected. The SFD for the analysis in this part is open-end without the consideration of fluid inertia effect. The $\pi$-film model is used. The geometries and operating conditions of the damper are listed in table 4.5 .

Table 4.4: Geometries of the Rotor (unit: inch)

\begin{tabular}{l|l|c}
\hline \multirow{3}{*}{ Shaft Geometry } & Diameter & 1 \\
& Axial Length & 24 \\
& Bearing Span & 18 \\
\hline \multirow{2}{*}{ Disk Geometry } & Diameter & 6 \\
& Thickness & 1 \\
\hline
\end{tabular}


Chapter 4 | Rotordynamics of Flexible Rotor Systems on SFDs using Force Coefficients80

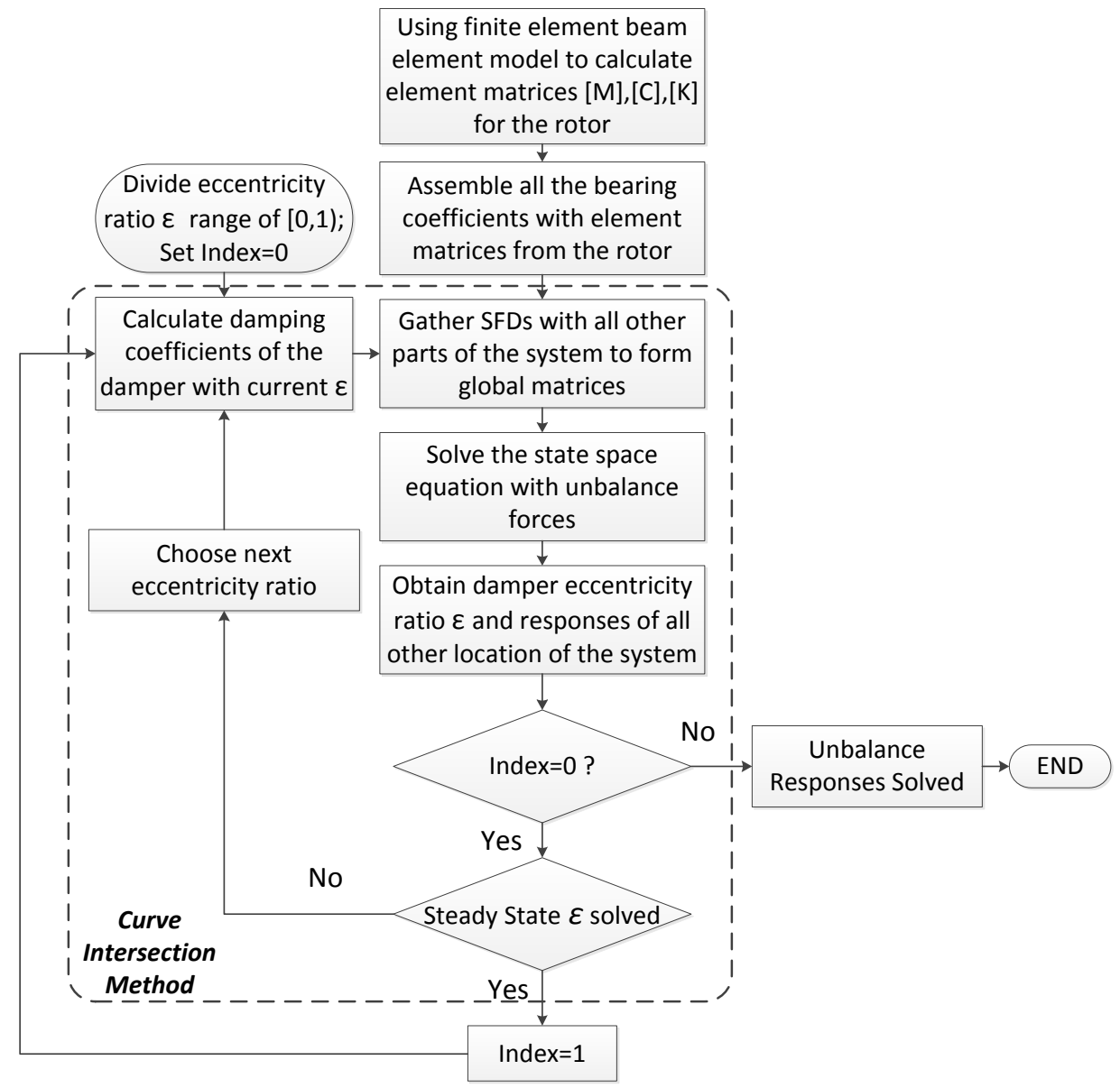

Figure 4.7: Scheme of Unbalance Responses Analysis with CIM

Figure 4.8 depicts the responses of the rotor at the location of left ball bearing, both cases with and without damper are included. The unbalance is located on disk 1 (the left disk) with phase angle of $0^{\circ}$. As shown by the figure, the response with the SFD is quite smooth through all the speed range from 1,000 to 10,000 rpm. Its peak response is about 25 times less than that of the response without SFD. Its amplification factor is 2.6, compared to 46.5 for the latter case. These observations demonstrate the effects of SFDs on the vibration elimination for rotor-bearing systems.

Figure 4.8 also shows that the SFD does not affect the responses with speeds below the first critical speed, however, it dissipates the vibration around the critical and gives a smooth transition through the critical area, which is desirable for reliable rotor-bearing systems. The two responses have different critical speeds. It is $4068 \mathrm{rpm}$ for the one with damper and 4375 
Table 4.5: Geometries and Operating Conditions of the Damper

\begin{tabular}{l|l}
\hline Journal Diameter $(D)$ & 3 in \\
\hline Axial Length $(L)$ & 0.375 in \\
\hline Radial Clearance $(C)$ & $2.7 / 1000$ in \\
\hline Centering Spring Stiffness $\left(K_{s}\right)$ & $5,000 \mathrm{lbf} /$ in \\
\hline Lubricant & ISO VG 32 \\
\hline
\end{tabular}

rpm for the other. The difference of $307 \mathrm{rpm}$ shows that the damper softens the support and produces less effective stiffness for the system. It is also worth noting that, despite the different locations of damper and ball bearing in Figure 4.8, the damper at the right end of the rotor is capable to diminish the vibration of the bearing at the other end.

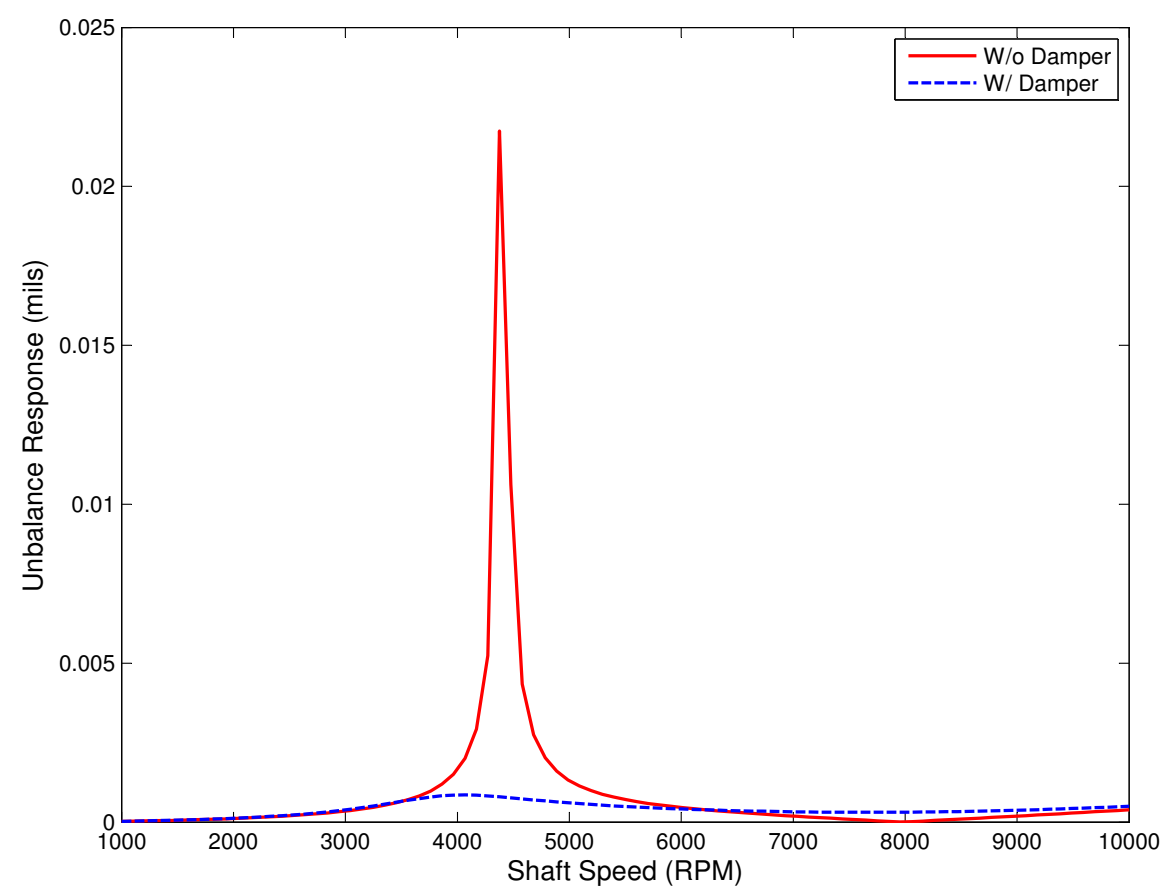

Figure 4.8: Comparison of Responses W/ and W/O Damper

Figure 4.9 shows the unbalance responses of the journal with three different unbalance locations. Each case has the unbalance force acting on one disk only. The magnitude and phase of the unbalance are fixed to $4 W / N$ and $0^{\circ}$, respectively. According to the API 684 [95], $W$ is the weight of the rotor when it is static; $N$ is the maximum running speed of the system with unit of rpm. From the figure, the unbalance on Disk 1(the left disk) has the lowest amplitude before and around the first critical speed. For the case on Disk 3 (the right 
Chapter 4 | Rotordynamics of Flexible Rotor Systems on SFDs using Force Coefficients82

disk), the response is the maximum before the critical speed range and becomes the second largest within the range. This happens because Disk 3 is the closest to the location of the damper, which generates the largest reacting force at the damper location with the same amount of unbalance. However, as the speed reaches the critical range, the unbalance force from the middle disk (Disk 2) is more effective in exciting the first bending mode of the system than the force on the right disk (Disk 3), and therefore gives the largest amplitude. In the figure, it also shows different location of the unbalance force excites three different first critical speeds, unlike the linear systems. In other words, the incorporation of SFD introduces nonlinearities to the original linear-system.

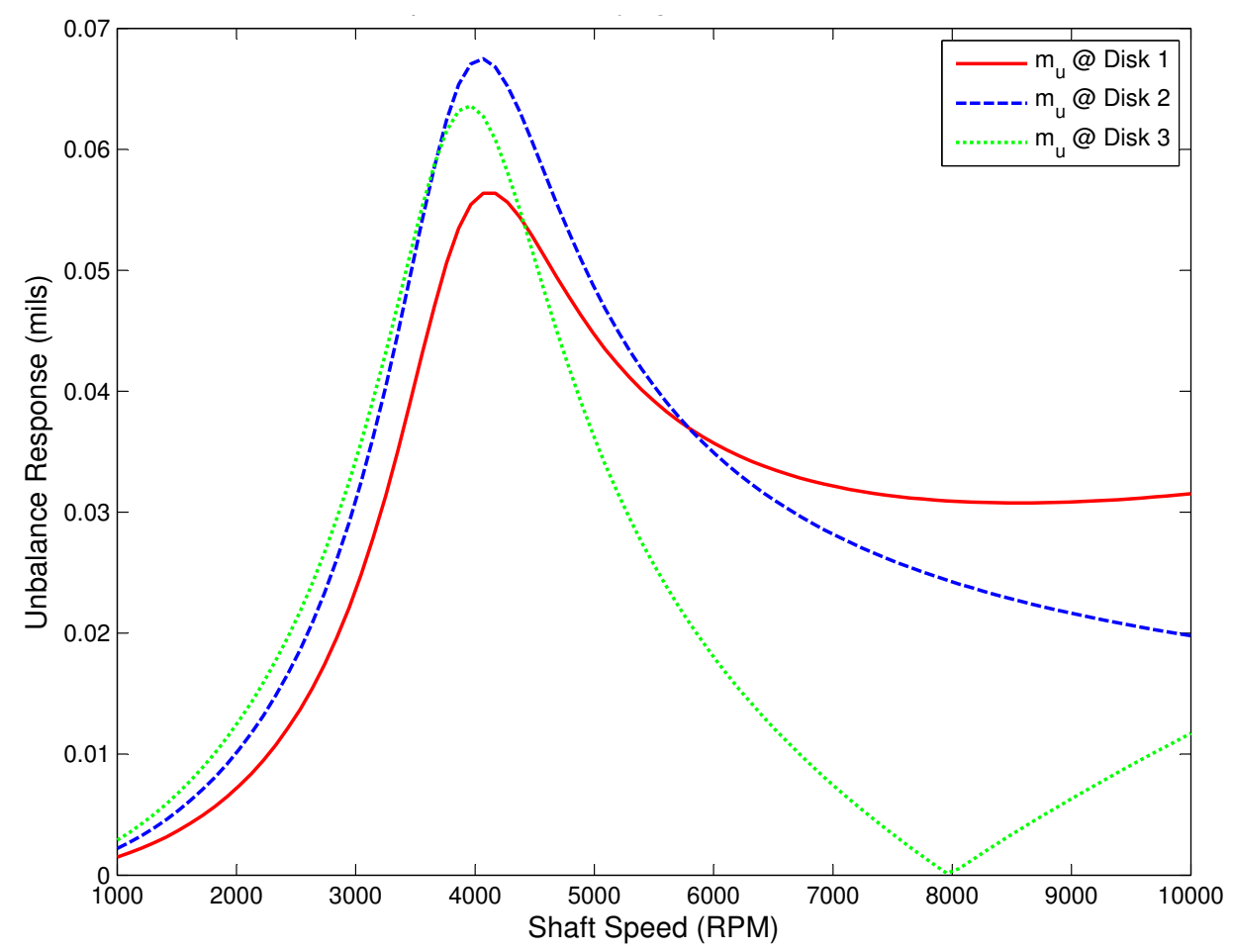

Figure 4.9: Responses of Damper Subject to Varying Unbalance Locations

Figure 4.10 gives the responses of the journal when the rotor is subject to unbalance at the middle disk (Disk 2) with magnitude changing from $4 W / N$ to $36 W / N$. The phase of the unbalance is $0^{\circ}$ for all the five cases. The choice of the location at middle disk is due to its effectiveness to excite the bending mode. As shown, the response is elevated through the whole speed range with larger unbalance, similar to linear systems. However, 


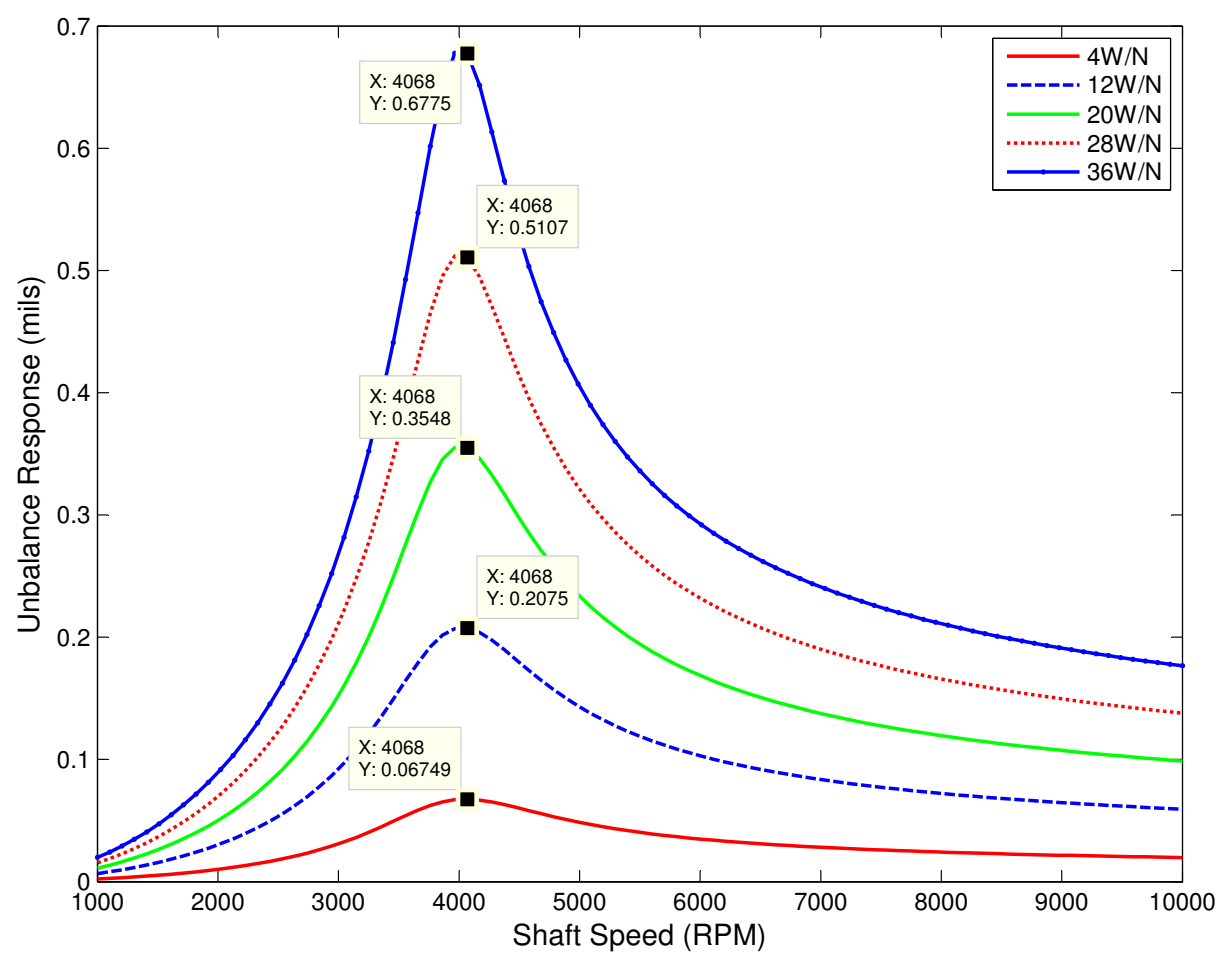

Figure 4.10: Responses of Damper Subject to Varying Unbalance on Disk 2

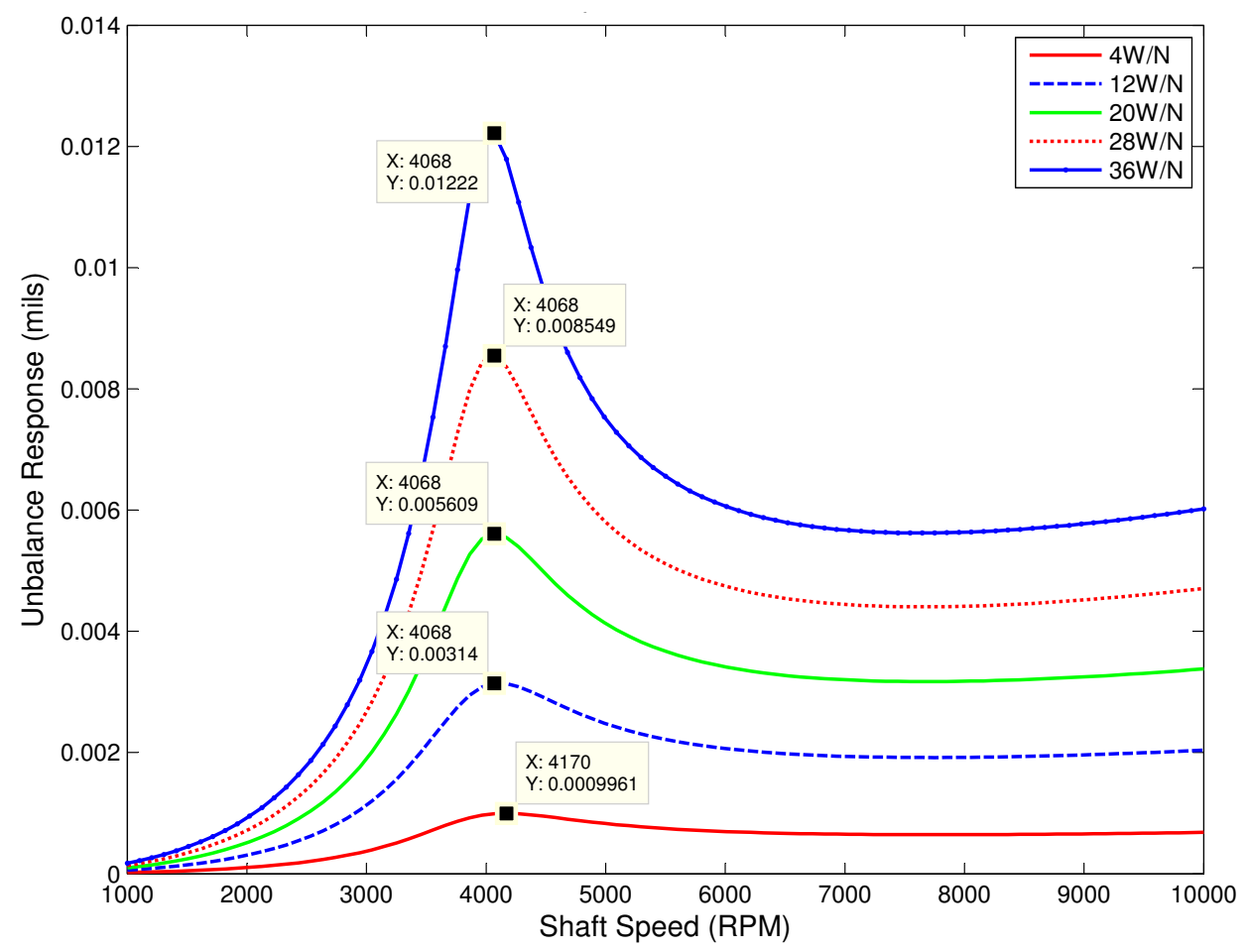

Figure 4.11: Responses of Left Bearing Subject to Varying Unbalance on Disk 2 
Chapter 4 | Rotordynamics of Flexible Rotor Systems on SFDs using Force Coefficients84

both the amplitude increment of responses and amplification factors near the critical speed behave differently from linear systems. The amplification factor increases from 2.6 to 3.7 as unbalance magnitude rises from $4 W / N$ to $36 W / N$. The growing amplification factor indicates the effective damping of the rotating systems is decreasing as unbalance increases. The amplitude increment at the critical speed is 0.14 mils as unbalance increases from $4 \mathrm{~W} / \mathrm{N}$ to $12 \mathrm{~W} / \mathrm{N}$, while for the same amount of raised unbalance, the amplitude increment is 0.17 mils from $28 \mathrm{~W} / \mathrm{N}$ to $36 \mathrm{~W} / \mathrm{N}$. These two observations make a rotating system with SFDs distinct from one without SFDs, where both the amplification factor and amplitude rise remain the same with linear variations of unbalance forces. Figure 4.11 shows the response of the rotor at the location of left ball bearing. It presents similar nonlinearities of the system.

\subsubsection{Responses with Varying Parameters of the SFD}

This part focuses on two important design parameters of SFD. They are clearance ratio $C / R$ and aspect ratio $L / D$. The unbalance force for the response is located at Disk 2 , with magnitude of $4 W / N$ and phase of $0^{\circ}$. The same rotor-bearing system as previous part is used with the same speed range. The changing range of the ratio is from $1 / 1000$ to $2.5 / 1000$.

The effects of clearance ratio on the responses of the journal are shown in Figure 4.12. It is found that higher clearance ratio produces larger responses at the location of journal. The peak amplitude with $C / R=2.5 / 1000$ is about $45 \%$ more than that with $C / R=1 / 1000$. In contrast to the responses of the journal, the right side ball bearing has the largest amplitude with the smallest clearance ratio, as shown in Figure 4.13. With the smallest ratio, the damper generates excessive damping, locks the journal precession and gives small vibration inside the damper. Due to the in-series connection with the damper, the vibration inside the ball bearing has to increase to react to the unbalance force from the rotor.

From Figure 4.12 and 4.13, the critical speed of the system decreases as the clearance ratio increases. This indicates the effective stiffness from the damper decreases with $C / R$. Different from the monotonic changes of the critical speed, the variation of amplification 


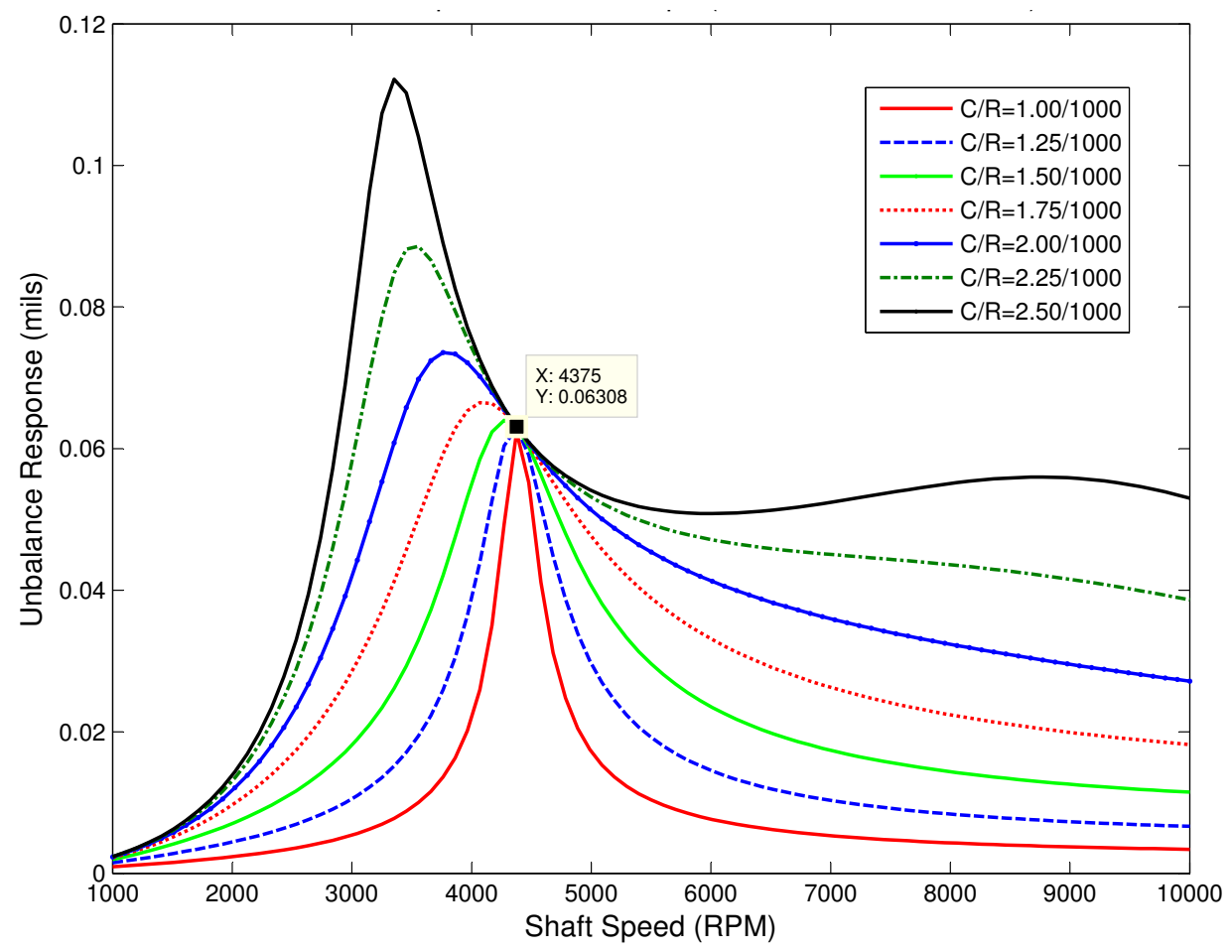

Figure 4.12: Responses of Damper Subject to Varying Clearance Ratio

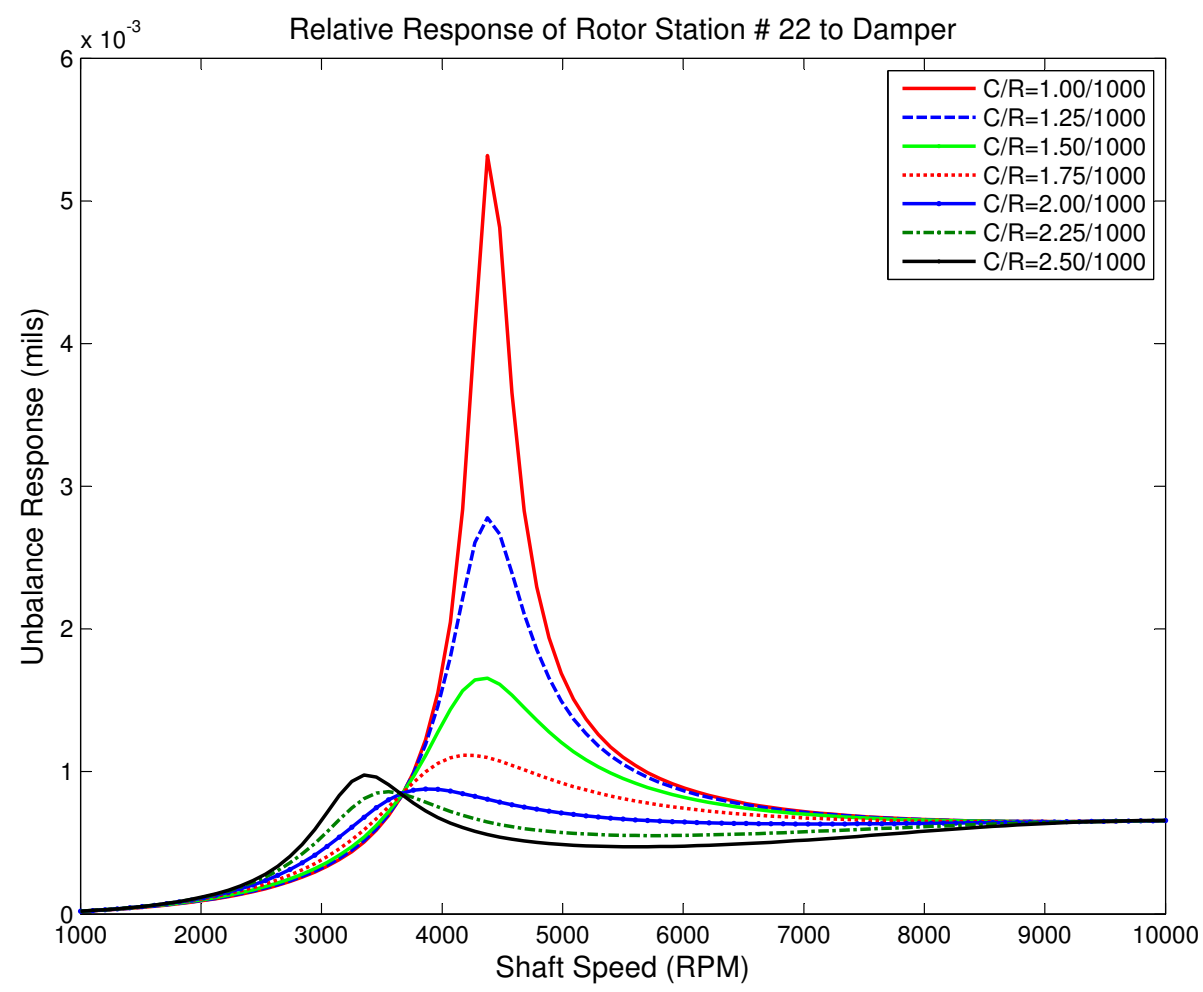

Figure 4.13: Responses of Right Ball Bearing with Varying Clearance Ratio 
Chapter 4 | Rotordynamics of Flexible Rotor Systems on SFDs using Force Coefficients86

factor is non-monotonic. It has the maximum value of 13.93 when $C / R=1 / 1000$, and reaches the minimum when the ratio is 2/1000, as shown in Figure 4.14. The amplification factor decreases with the ratio for the range of $[1 / 1000,2 / 1000]$ and increases when the ratio is larger than 2/1000. Hence, it is possible for two different clearance ratios to give the same amplification factor, based on the parabolic relation shown in Figure 4.14. From the stability point of view, the minimum amplification factor when $C / R=2 / 1000$ is desirable as it gives the maximum effective damping to diminish vibration from the journal.

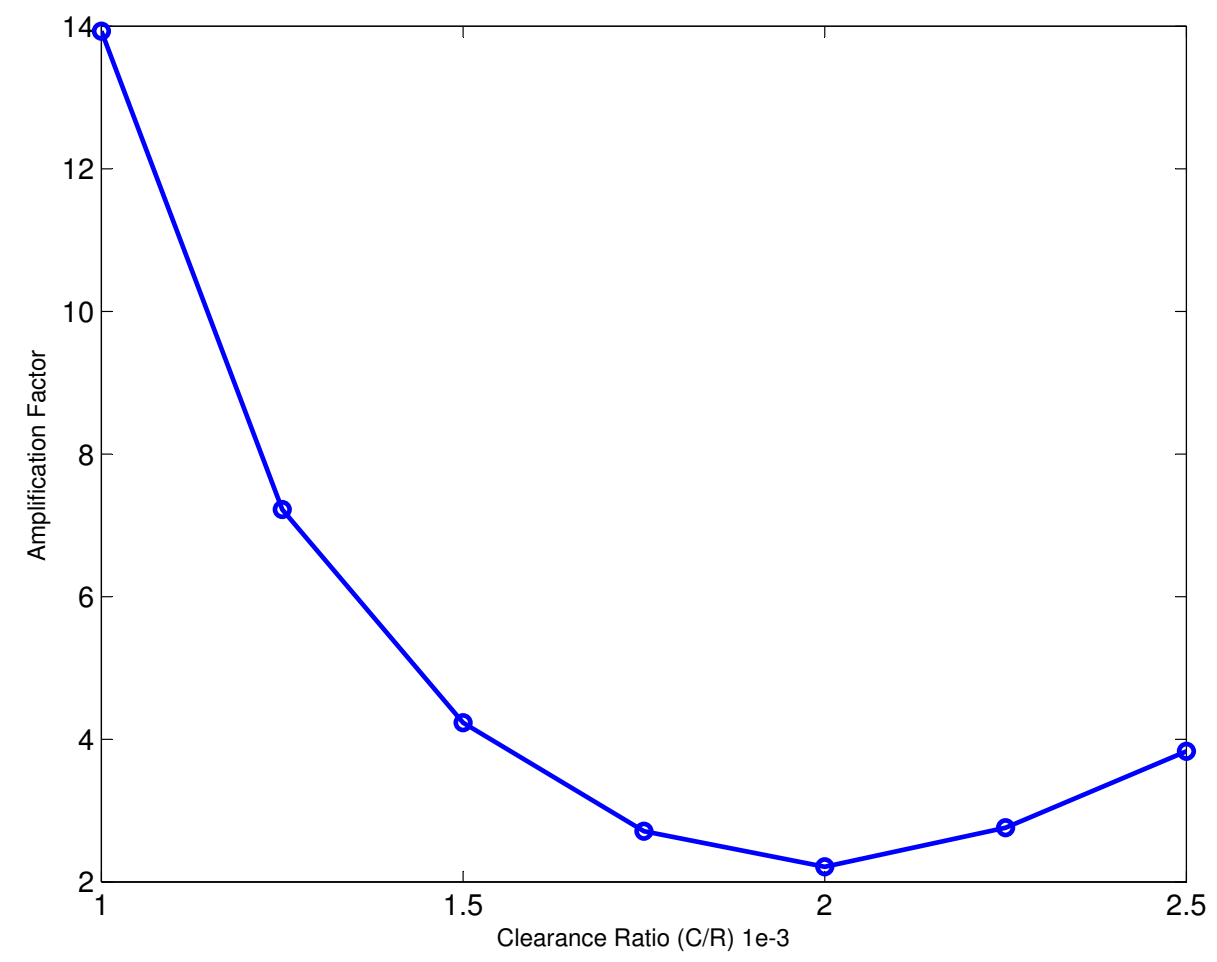

Figure 4.14: Amplification Factor V.S. Clearance Ratio

The aspect ratio is another important factor related to the damping capacity of SFDs. Within this study, the clearance ratio equals to $1.8 / 1000$ for all the variation of aspect ratio. All the other conditions including unbalance, damper radius and centering stiffness are kept unchanged. Figure 4.15 shows the journal responses subject to the variation of aspect ratio. Due to the assumptions of short-bearing and open-end for dampers in this chapter, the minimum value of the variation is 0.075 . And according to [96], the short-bearing theory can 
not predict accurately when the aspect ratio is larger than $1 / 4$. Therefore, the upper limit of the range is chosen as 0.225 .

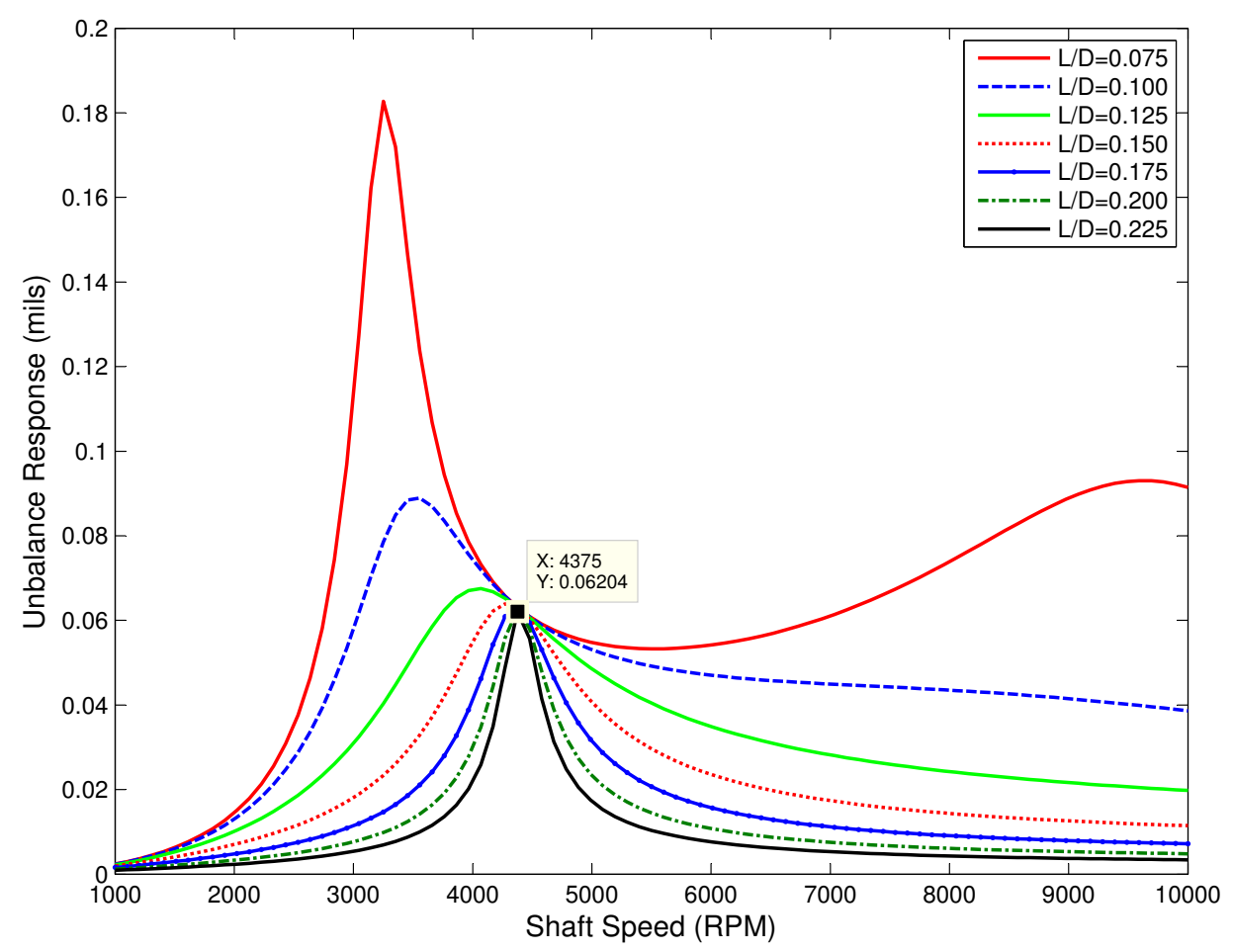

Figure 4.15: Responses of Damper with Varying Aspect Ratio

Compared to the effects of clearance ratio on the damper responses in Figure 4.12, the shape and trends of responses subject to the variation of aspect ratio are quite similar. However, the relation between the aspect ratio and the responses is different from that between clearance ratio and the responses. The larger aspect ratio gives higher critical speed and reduces the amplitude at the same time. The amplification factor reaches the minimum value of 2.58 when the ratio is $1 / 8$. Figure 4.15 depicts the relative displacement of the right ball bearing subject to the variation of aspect ratio. The largest $L / D$ gives the highest amplitude and largest critical speed. 
Chapter 4 | Rotordynamics of Flexible Rotor Systems on SFDs using Force Coefficients88

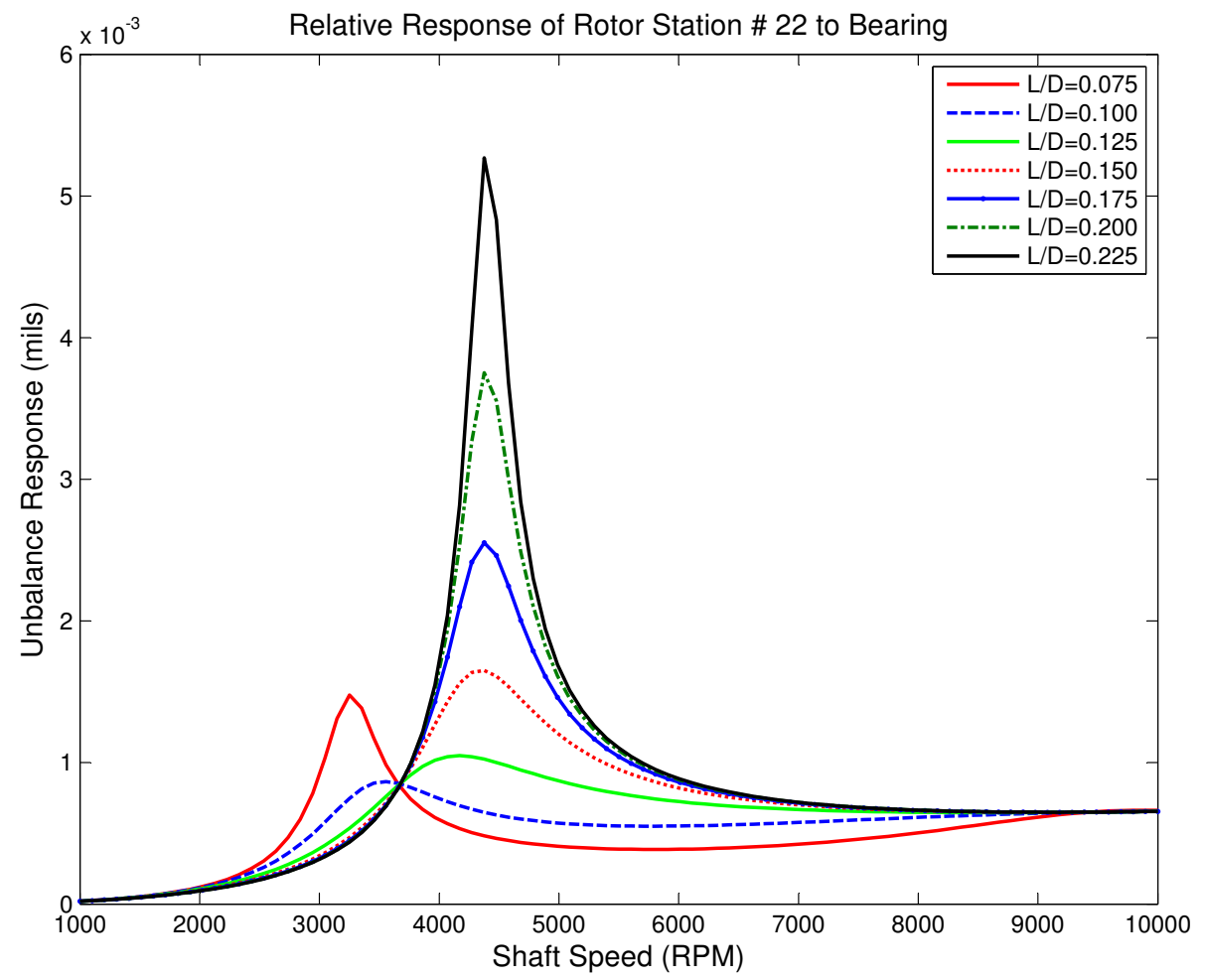

Figure 4.16: Responses of Right Ball Bearing with Varying Aspect Ratio

\subsection{Stability Analysis Using Force Coefficients}

With obtained steady state solutions with given unbalance forces, the stability of the rotorbearing system can be evaluated. The main assumption for this analysis is that, the SFD behaves linearly surrounding its equilibrium position when subject to unbalance forces. Therefore, its damping and effective stiffness properties from the responses will not change for the stability analysis. This section starts with a brief introduction of the mathematical model for stability analysis with SFDs, then applies it to the flexible bearing systems as described before. Different rotor operating conditions with various parameters of the SFD are included.

\subsubsection{Mathematical Model for Stability Analysis with SFDs}

The stability analysis here is focused on the self-excited vibration analysis when the rotorbearing system is under steady state. Several literatures have covered detailed derivations for 
the stability analysis $[92,97,98,99]$. Therefore, this part only gives brief introduction about the analysis.

Different from the equation 4.17 in part 4.4.1, the right hand side of the equation should be zero, which gives,

$$
\left[\begin{array}{cc}
\mathbf{M}_{s} & \mathbf{0} \\
\mathbf{0} & \mathbf{M}_{s}
\end{array}\right]\{\dot{u}\}_{s}+\left[\begin{array}{cc}
\mathbf{0} & -\mathbf{M}_{s} \\
\mathbf{K}_{s} & \overline{\mathbf{C}}_{s}
\end{array}\right]\{u\}_{s}=\left[\begin{array}{l}
0 \\
0
\end{array}\right]
$$

where, the subscript $s$ represents the steady state. $\mathbf{M}_{s}, \mathbf{K}_{s}$ and $\overline{\mathbf{C}}_{s}$ are the mass, stiffness and damping (from both the damper and gyroscopic effects) matrix under the equilibrium status.

Introducing the similar displacement form to the unbalance response analysis gives the steady state form as,

$$
\{u\}_{s}=\{\mathbf{U}\}_{s} e^{\lambda t}
$$

where, $\lambda=\alpha+j \omega$

Substituting above equation to the equation 4.23 and dividing both sides by $e^{\lambda t}$ yields,

$$
\lambda\left[\begin{array}{cc}
\mathbf{M}_{s} & \mathbf{0} \\
\mathbf{0} & \mathbf{M}_{s}
\end{array}\right]\{\mathbf{U}\}_{s}+\left[\begin{array}{cc}
\mathbf{0} & -\mathbf{M}_{s} \\
\mathbf{K}_{s} & \overline{\mathbf{C}}_{s}
\end{array}\right]\{\mathbf{U}\}_{s}=\left[\begin{array}{l}
0 \\
0
\end{array}\right]
$$

The above equation can be transferred into a standard eigenvalue problem as,

$$
\{\lambda \mathbf{I}+\mathbf{W}\}\{\mathbf{U}\}_{s}=\mathbf{0}
$$

where,

$$
\mathbf{W}=\left[\begin{array}{cc}
\mathbf{M}_{s} & \mathbf{0} \\
\mathbf{0} & \mathbf{M}_{s}
\end{array}\right]^{-1}\left[\begin{array}{cc}
\mathbf{0} & -\mathbf{M}_{s} \\
\mathbf{K}_{s} & \overline{\mathbf{C}}_{s}
\end{array}\right]
$$

To this point, the stability of the steady state can be revealed by solving the complex eigenvalue problem for equation 4.26. Several commercial computational tools are available to solve the problem. 
Chapter 4 | Rotordynamics of Flexible Rotor Systems on SFDs using Force Coefficients90

\subsubsection{Stability with Varying Operating Conditions of the Rotor}

The rotor-bearing system is the same as described in section 4.4.2. Details about the rotor diameter and damper configuration can be found in table 4.7 and 4.5 , respectively. The damper is assumed to be open-end with cavitation $(\pi$-film).

Table 4.6: Frequency and Log Dec Comparison, W/ and W/O SFD

\begin{tabular}{l|l|l}
\hline & Natural Frequency $(c p m)$ & Log Dec $(\delta)$ \\
\hline$W / O$ SFD & 4248.5 & $7.76 \mathrm{e}-13$ \\
\hline$W / \mathrm{SFD}$ & 3920.5 & 1.23 \\
\hline
\end{tabular}

Table 4.6 compares the natural frequency $N_{c}$ and logarithm decrement $\delta$ of the rotorbearing with and without the damper. The operating speed is $8000 \mathrm{rpm}$, with the unbalance excitation at the middle disk with magnitude of $4 W / N$ and phase of $0^{\circ}$. Only the first forward bending mode is listed in the table. As shown, the original rotor without SFD is marginally stable, according to control theory [100]. ${ }^{1}$ However, the incorporation of SFD improves the stability greatly by increasing the log decrement to 1.23 , which satisfies the API 684 . The natural frequency decreases about $300 \mathrm{rpm}$ from the original rotor-bearing system. Both the change of log decrement and natural frequency in the table are in accordance with the unbalance responses shown in Figure 4.8.

Figure 4.17 depicts the first bending mode shapes for cases with and without SFD. The main difference between these two is the displacement at the right end of the rotor, where the SFD is located. The assembling of the SFD allows much more motion at the bearing node, compared to the locked node in 4.17(a). This large motion indicates the journal moves inside the SFD effectively, which generates desired damping to stabilize the rotating system.

Cross-coupled stiffness has great effects on the stability of rotating machines[98, 101]. Squeeze film damper is one of the solutions to diminish the potential instability issue due to cross-coupled stiffness $[17,102]$. Figure 4.18 shows the effect of cross-coupled stiffness on the

\footnotetext{
${ }^{1}$ however, according to the API 684 [95], its stability is an issue because the log decrement is much smaller than 0.2 .
} 


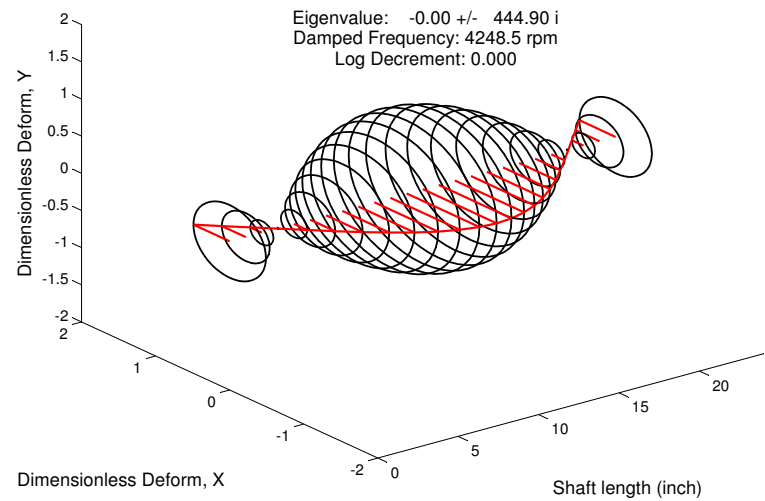

(a) Without SFD

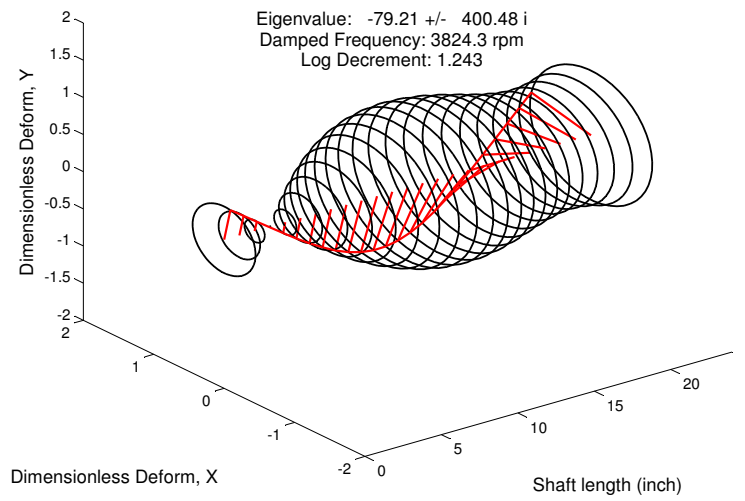

(b) With SFD

Figure 4.17: Mode Shape Comparison at 8000 rpm

stability of current rotor-bearing system. The cross-coupled stiffness is applied on the left disk. Only one speed case, 8000 rpm, is studied here.

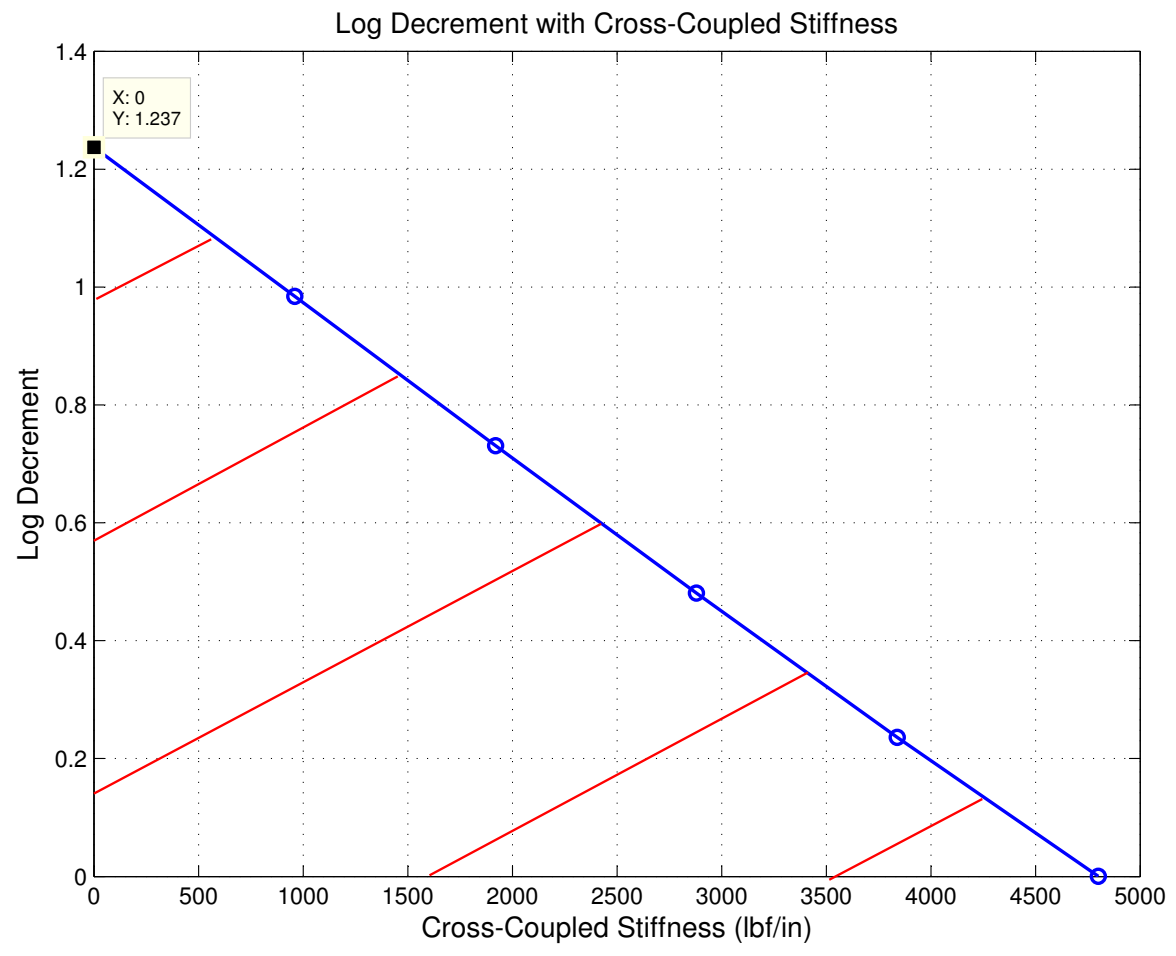

Figure 4.18: Log Decrement V.S. Cross-Coupled Stiffness

As shown in the figure, the log decrement(stability margin) decreases with cross-coupled stiffness. It is 1.237 when no cross-coupled stiffness is applied and decreases to zero when the stiffness is around 4,800 $\mathrm{lbf} / \mathrm{in}$. The relation between log decrement and the stiffness is linear 
Chapter $4 \quad$ Rotordynamics of Flexible Rotor Systems on SFDs using Force Coefficients92

for current rotor-bearing system. Caution must be paid to generalize this linear relation to other systems. In [103], the same rotor with tilting pad journal bearings gives a parabolic relation. Triangle area with red line shows the effectiveness of SFD on the improvement of system stability. As discussed at the beginning of this section, the rotor-bearing system without SFD is unstable if any cross-coupled stiffness is present. This is because the log decrement of the system is zero. In other words, the stability margin increases from $0 \mathrm{lbf} / \mathrm{in}$ to $4,800 \mathrm{lbf} / \mathrm{in}$, if SFD is present.

\subsubsection{Stability with Varying Parameters of the SFD}

The effects of two parameters, the clearance ratio $C / R$ and aspect ratio $L / D$, on system stability are documented in this part. The operating conditions are the same as those discussed in part 4.4.3 for unbalance responses analysis. The variation range of $C / R$ and $L / D$ are $[1 / 1000,2.5 / 1000]$ and $[0.075,0.225]$, respectively.

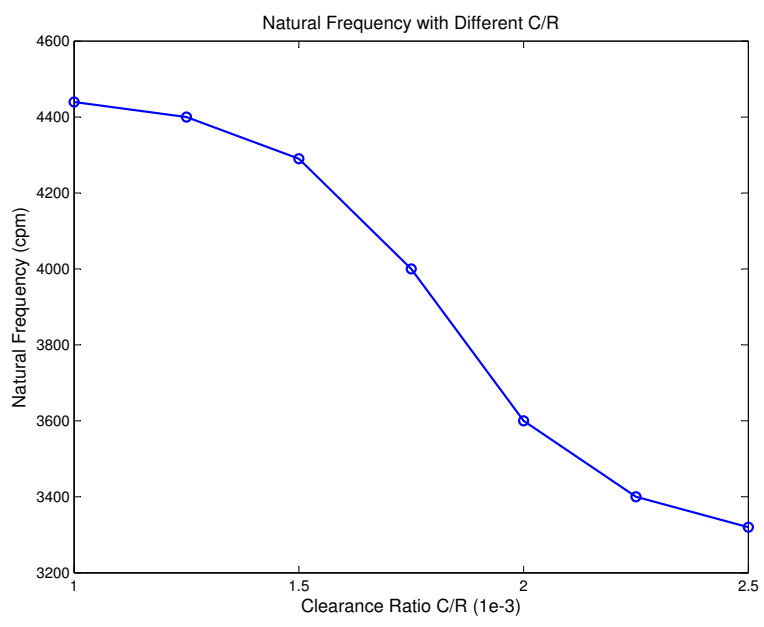

(a) Natural Frequency V.S. $C / R$

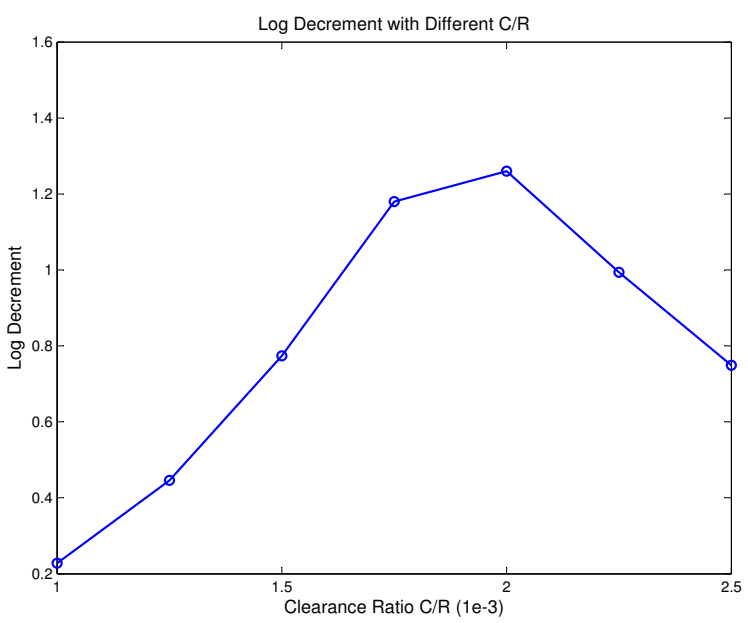

(b) Log Decrement V.S. $C / R$

Figure 4.19: Stability with Varying $C / R$ at 8000 rpm

The natural frequency and $\log$ decrement with the variation of $C / R$ are shown in Figure 4.19. The aspect ratio $L / D$ is fixed to $1 / 8$ and the running speed is $8000 \mathrm{rpm}$ during the variation. From Figure 4.19(a), the increase of clearance ratio decreases the frequency from $4439 \mathrm{rpm}$ to $3320 \mathrm{rpm}$, about $1120 \mathrm{rpm}$ reduction for the whole variation range. This 
observation implies the smaller clearance ratio the stiffer the SFD. Within the variation range, the natural frequency does not decrease with clearance ratio linearly. The decreasing rate for the middle range of $[1.5 / 1000,2 / 1000]$ is larger than that of the rest.

Figure 4.19(b) gives the relation between log decrement and clearance ratio. Different from natural frequency, the relation is not a monotonous function, since the log decrement increases with the ratio first before decreasing. It has the maximum value of 1.26 when $C / R$ equals to $2 / 1000$, and two different clearance ratios might give the same log decrement.

The effects of aspect ratio on the natural frequency and log decrement are depicted in Figure 4.20. The clearance ratio equals 1.8/1000 and running speed is $8000 \mathrm{rpm}$. Different from Figure 4.19(a), the natural frequency increases with aspect ratio and gives about 1180 rpm variation between the maximum and minimum. The increasing rate is the largest for the middle range of $[0.10,0.15]$. For the ratio larger than 0.15 , it can not raise the frequency effectively. The change between $[0.20,0.225]$ only results in $10 \mathrm{rpm}$ increment.

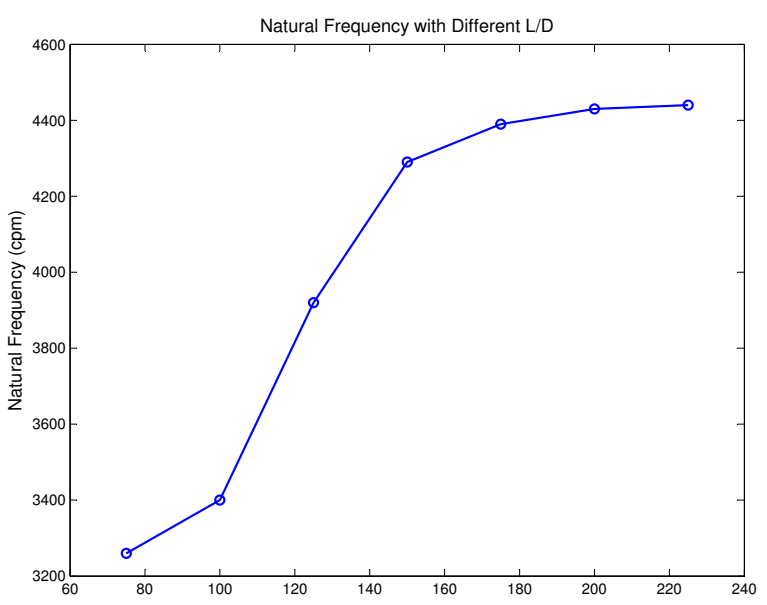

(a) Natural Frequency V.S. $L / D$

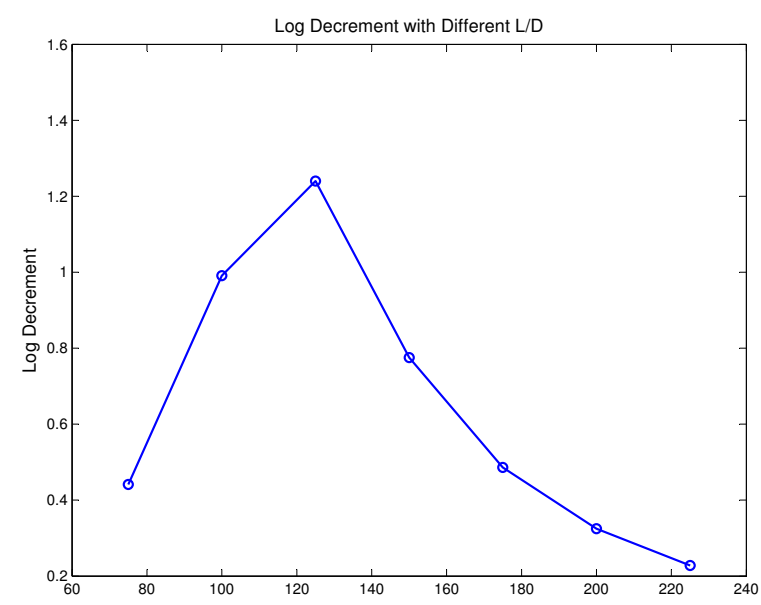

(b) Log Decrement V.S. $L / D$

Figure 4.20: Stability with Varying $L / D(\mathrm{e}-3)$ at 8000 rpm

Figure 4.20(b) represents the relation between log decrement and aspect ratio. Similar to the relation in Figure 4.19(b), it increases with $L / D$ and reaches the maximum value before decreasing. The ratio of 0.125 gives the largest value of 1.24. From the figure, the increasing and decreasing rate are different from each other. The log decrement is found 
Chapter 4 | Rotordynamics of Flexible Rotor Systems on SFDs using Force Coefficients94

more sensitive to the $L / D$ variation between 0.075 and 0.125 than that for the range above. Within the variation range with high aspect ratio, the increasing $L / D$ becomes less effective in the reduction of the log decrement. Comparing these observations with the results in unbalance analysis, they are correlated well with each other.

\subsection{Summary}

This chapter documented the rotordynamics analysis of rotor-bearing system with SFDs using force coefficients method. The implicit relation between journal dynamics and damper reacting forces was discussed. A new method, the Curve Intersection Method, was created to find the steady state solution. It is more robust and effective than traditional iteration methods for unbalance responses of rotor-bearing systems with SFDs. A code based on this method was developed to realize both unbalance response analysis and stability analysis.

The effects of SFDs on the rotordynamics of a rotating system were studied by applying the developed tool to a flexible rotor-bearing system. Both unbalance response and stability of the system were investigated when subjected to different operating conditions. The location and magnitude variation of unbalance forces were found to have more influence on unbalance responses than on system stability. The larger unbalance gives higher responses and higher amplification factor, therefore, the stability margin decreases. All these relations observed are nonlinear.

The influence of two important damper parameters, the clearance ratio and aspect ratio,

on both unbalance response and stability were documented. Both the clearance ratio of $2 / 1000$ and aspect ratio of $1 / 8$ were found to have the most desirable performance of the current rotating system. 


\section{Chapter 5}

\section{Rotordynamics of Flexible Rotor}

\section{Systems on SFDs using Direct Force}

Although the force coefficients method is simple and effective for some rotordynamics analyses with SFDs, its applications are limited by the assumptions used to obtain the damping coefficients. These assumptions include small aspect ratio(short bearing), $\pi$-film cavitation with open-end, $2 \pi$-film without cavitation, or zero pressure within supply/discharge grooves. In addition, the force coefficients method also prevents the journal from free precession, requiring either a synchronous Centered Circular Orbit(CCO) or a synchronous Off-center Circular Orbit(OCO) with small amplitude.

The above assumptions reduce the usefulness of the force coefficients method for realistic rotor-bearing systems in industrial applications and make direct force methods desirable. The direct force methods, no damping coefficients are required in the modeling. The incorporation of SFDs into the rotor-bearing system is realized by considering the damper forces directly. Hence, it removes the simplifying assumptions that are necessary to go from damper forces to damping coefficients in the force coefficients method.

The damper forces can be considered in either the time domain or the frequency domain for the rotordynamics analysis with SFDs. Methods in the time domain include the Runge- 
Kutta method[104], Newmark- $\beta$ method[105], and shooting method [106] among others. The frequency domain methods include the harmonic balance method [107], trigonometric collocation method[26], hybrid numerical method [72].

Taylor [66] and Cookson [108] modeled the damper forces with a fourth order Runge-Kutta integration method to find the nonlinear response of a rotor-bearing system supported on centered, short dampers and off-centered dampers, respectively. With the same procedure, Sykes and Holrnes [21] characterized the effects of bearing misalignment on the response of a damper supported rotor, and captured the nonlinear jump phenomenon under different cavitation pressures.

As discussed in chapter 1 and chapter 2, the methods in the time domain are not computationally efficient compared to the frequency domain methods. They focus more on the nonlinear transient than the steady state solutions. Since the steady state rotordynamics analysis is the concentration of the current work, the direct force methods in the time domain are not the first choices. Instead, the direct force methods in the frequency domain are employed. The harmonic balance method, due to its simplicity and effectiveness, is used in this dissertation to obtain the steady state solutions of flexible rotating systems with SFDs.

Several previous works related to rotating systems on SFDs have used the harmonic balance method [23, 24, 25]. However, these studies either consider only simple rotor-bearing systems having few DOF or neglect important effects such as gyroscopics and excessive unbalance. Hence, these existing methods are not able to model practical rotating systems with SFDs. This chapter develops an improved harmonic balance method to satisfy the practical needs.

Firstly, a harmonic balance method applied to a reduced equation of motion is developed. Both the forced response and stability analysis using this method are included. Then, a predictor-corrector procedure based on the method is investigated to obtain forced responses of the rotating system under varying speeds with high nonlinearities. The rotordynamics analysis of a flexible rotor with SFDs is then studied to validate the improved harmonic 
balance method for nonlinear rotating systems.

\subsection{The Harmonic Balance Method}

From the brief introduction of harmonic balance method in part 2.4.2, the displacement form of steady state solutions of the nonlinear system can be expressed as,

$$
x(t)=X_{0}+\sum_{i=1}^{k}\left[X_{c i} \cos \left(\omega_{i} t\right)+X_{s i} \sin \left(\omega_{i} t\right)\right],
$$

In rotordynamic analyses, the harmonic component $\omega_{i}$ is related to the running speed. Sub and super components are possible, dependent on the operating conditions of the rotating system. The number of $\omega_{i}$ is determined by $k$ in the above equation. Note that equation 5.1 is the displacement for one Degree-Of-Freedom(DOF) only. Vector format is required for multiple DOF system.

Different from the existing harmonic balance method used by other works with their simple rotor-bearing systems $[23,24,109]$, the finite element method is adopted in order to model realistic rotor-bearing systems having high DOF. Similar to the analysis in chapter 4, the equation of motion is given as follows,

$$
[\mathbf{M}]\{\ddot{\mathbf{x}}\}+([\mathbf{C}]+\Omega[\mathbf{G}])\{\dot{\mathbf{x}}\}+[\mathbf{K}]\{\mathbf{x}\}=\{\mathbf{f}(\mathbf{x})\}
$$

Note that, the matrices of $[\mathbf{C}]$ and $[\mathbf{K}]$ on the left side do not include the coefficients contributed by SFDs. Different from the force coefficients method in the previous chapter, the force vector on the right side includes not only the unbalance forces from the rotor, but also the forces from SFDs, gravity of the rotating system and other external loads. Substituting the vector format of equation 5.1 to equation 5.2 will give the equation to solve the unknowns in the frequency domain. 


\subsubsection{Forced Responses Using Harmonic Balance Method}

The equations to obtain the force responses are available once the substitution of 5.1 to equation 5.2 is completed. Three sub-equations will be available to form the new equation of motion in the frequency domain, shown as follows,

$$
\begin{aligned}
& {[\mathbf{K}]\left\{\mathbf{X}_{\mathbf{0}}\right\}=\widetilde{\mathbf{F}}(\mathbf{X})_{\text {constant }}} \\
& \left([\mathbf{K}]-\omega_{\mathbf{i}}^{2}[\mathbf{M}]\right)\left\{\mathbf{X}_{\mathbf{c i}}\right\}+\omega_{i}([\mathbf{C}]+\Omega[\mathbf{G}])\left\{\mathbf{X}_{\mathbf{s i}}\right\}=\widetilde{\mathbf{F}}(\mathbf{X})_{\cos } \\
& \left([\mathbf{K}]-\omega_{\mathbf{i}}^{2}[\mathbf{M}]\right)\left\{\mathbf{X}_{\mathbf{c i}}\right\}-\omega_{i}([\mathbf{C}]+\Omega[\mathbf{G}])\left\{\mathbf{X}_{\mathbf{s i}}\right\}=\widetilde{\mathbf{F}}(\mathbf{X})_{\sin }
\end{aligned}
$$

It is not hard to find that the three sub-equations result from constant, cosine and sine terms of Fourier transformation. It is worth noting that $\omega_{i}$ is different from $\Omega$. The former is the vibration frequency of the rotating system, and the latter is the operating speed. If $\omega_{i}$

equals to $\Omega$, then it is called synchronous vibration. However, $\omega_{i}$ can be other frequencies, such as sub- and super- harmonics depending on the operating conditions. $\Omega$ is a fixed running speed in this part. For the varying speed range, it will be discussed in section 5.2.

Same as the equation of motion using force coefficients method, the above equation in frequency domain is an implicit equation. Therefore, iteration method such as NewtonRaphson is required to get the solutions. Current total number of unknowns to be solved iteratively is $(2 k+1) n$, where $n$ is the number of DOF of the whole system. Compared to the time domain method, the number of unknowns rises from $n$ to $(2 k+1) n$, which enlarges the computational burden $(2 k+1)$ times. To remove this disadvantage of the traditional harmonic balance method, the current work introduces a complex vector and separates the linear and nonlinear part of the system.

The idea of linear and nonlinear separation comes from the observation of implicity relation in the above equation of motion. It is the nonlinear part of the system that requires iteration procedures. This is because the implicit relation in equation 5.3 only exists between the dynamics of the journal and its corresponding forces. Other than the nodes of SFDs, the iteration is not necessary. Hence, if it is possible to reduce the number of unknowns requiring 
iteration, the computational efficiency will be improved dramatically.

To separate the linear and nonlinear parts of the system, a complex vector $\widetilde{\mathbf{R}}$ is introduced as,

$$
\widetilde{\mathbf{R}}= \begin{cases}\mathbf{X}_{0} & i=0, \\ \mathbf{X}_{c i}+j \mathbf{X}_{s i} & i=1, \ldots, k\end{cases}
$$

Re-arranging equation 5.3 with $\widetilde{\mathbf{R}}$ and separating linear and nonlinear parts of the system gives the following equation for each harmonic component as,

$$
\left[\begin{array}{ll}
\mathbf{P}_{\mathbf{1 1}} & \mathbf{P}_{\mathbf{1 2}} \\
\mathbf{P}_{\mathbf{2 1}} & \mathbf{P}_{\mathbf{2 2}}
\end{array}\right]_{i}\left\{\begin{array}{c}
\widetilde{\mathbf{R}}_{1} \\
\widetilde{\mathbf{R}}_{2}
\end{array}\right\}_{i}=\left\{\begin{array}{c}
\widetilde{\mathbf{F}}_{1}^{L} \\
\widetilde{\mathbf{F}}_{2}^{L}
\end{array}\right\}_{i}+\left\{\begin{array}{c}
\mathbf{0} \\
\widetilde{\mathbf{F}}_{2}^{N L}
\end{array}\right\}_{i}
$$

where, the subscript 1 and 2 represent linear and nonlinear part, respectively. The superscript $L$ represents linear force and $N L$ is nonlinear force. For example, $\widetilde{\mathbf{F}}_{2}^{L}$ describes the linear force vector acting on the nonlinear parts of the system. $\left[\mathbf{P}_{\alpha \beta}\right]_{i}$ equals to following equation,

$$
\left[\mathbf{P}_{\alpha \beta}\right]_{i}=\left[\mathbf{K}_{\alpha \beta}\right]_{i}-\omega_{i}^{2}\left[\mathbf{M}_{\alpha \beta}\right]_{i}-j \omega_{i}\left[\mathbf{C}_{\alpha \beta}\right]_{i}
$$

with $\alpha$ and $\beta$ equal to $1,2 .\left[\mathbf{M}_{\alpha \beta}\right],\left[\mathbf{K}_{\alpha \beta}\right]$ and $\left[\mathbf{C}_{\alpha \beta}\right]$ are the mass, stiffness and damping matrix corresponding to linear and nonlinear part, respectively.

With separated equation 5.5, the displacement of nonlinear part $\widetilde{\mathbf{R}}_{2}$ can be solved,

$$
\left\{\widetilde{\mathbf{R}}_{2}\right\}_{i}=[\mathbf{U}]_{i}^{-1}\left(\left\{\widetilde{\mathbf{F}}_{2}^{N L}\right\}_{i}-\{\mathbf{V}\}_{i}\right)
$$

where,

$$
\begin{aligned}
& {[\mathbf{U}]=-\left[\mathbf{P}_{\mathbf{2 1}}\right]\left[\mathbf{P}_{11}\right]^{-1}\left[\mathbf{P}_{\mathbf{1 2}}\right]+\left[\mathbf{P}_{\mathbf{2 2}}\right]} \\
& \{\mathbf{V}\}=\left[\mathbf{P}_{\mathbf{2 1}}\right]\left[\mathbf{P}_{11}\right]^{-1}\left\{\mathbf{F}_{\mathbf{L}}^{\mathbf{1}}\right\}-\left\{\mathbf{F}_{\mathbf{L}}^{\mathbf{2}}\right\}
\end{aligned}
$$

Equation 5.7 is the one that needs to be solved iteratively. However, the number of unknowns requiring iteration reduced from $(2 k+1) n$ to $(k+1) N$, where $N$ is the number of 
DOF of nonlinear parts only. In realistic rotating systems, the DOF number of nonlinear parts is much less than that of the overall system. If a system is supported on two SFDs, then the nonlinear parts only have four DOF. Therefore, the efficiency of computation is improved dramatically by the separation of the improved harmonic balance method. More details about the separation can be found in Appendix A. Once $\left\{\widetilde{\mathbf{R}}_{2}\right\}$ is obtained, the displacement of linear parts $\left\{\widetilde{\mathbf{R}}_{1}\right\}$ is available readily from equation 5.5 without any iteration steps. The displacement of each DOF in the time domain can be obtained using equation 5.1.

\subsubsection{Stability Analysis Using Harmonic Balance Method}

Typical methods for the stability evaluation include Floquet theory [110] and Poincaré map [111]. These two methods are applied after obtaining the steady sate responses from the harmonic balance method. They have been used in several works related to the rotordynamics of rotating system on $\operatorname{SFDs}[24,25,27,60,74,112]$. It is worth noting that both Floquet theory and Poincaré map are the methods in the time domain, different from the domain within which the steady state solution is obtained. The switch from the frequency domain to time domain make these two methods not effective for the computation.

Different from previous works, the current work keeps consistent within the same domain as forced response analysis. The main reason is that the forced response analysis using harmonic balance method gives important information for stability evaluation, the Jacobian matrix, when solving nonlinear algebra equations iteratively (section 5.2 shows more details). The Jacobian matrix has contained the stability information of the steady state solution already. Thus, it is unnecessary to start the calculation over in the time domain. A perturbation method is applied to evaluate system stability using the existing Jacobian matrix.

The perturbed displacement $\mathbf{x}(\mathbf{t})$ around the steady state $\mathbf{x}_{\mathbf{e}}(\mathbf{t})$ can be expressed as,

$$
\mathbf{x}(\mathbf{t})=\mathbf{x}_{\mathbf{e}}(\mathbf{t})+\Delta \mathbf{x}(\mathbf{t})
$$


with,

$$
\Delta \mathbf{x}(\mathbf{t})=e^{\lambda t} \mathbf{q}(\mathbf{t})
$$

where, $\mathbf{q}(\mathbf{t})$ has the Fourier expansion as,

$$
\mathbf{q}(\mathbf{t})=\mathbf{Q}_{\mathbf{0}}+\sum_{i=1}^{k}\left[\mathbf{Q}_{\mathbf{c i}} \cos \left(\omega_{i} t\right)+\mathbf{Q}_{\mathbf{s i}} \sin \left(\omega_{i} t\right)\right]
$$

For convenience, the subscript $i$ is neglected for the following derivation. Substituting 5.10 into equation 5.2 and collecting constant, cosine and sine terms give,

$$
\left(\begin{array}{cc}
\widetilde{\mathbf{K}} & \mathbf{K}^{*}
\end{array}\right)\left(\begin{array}{c}
\mathbf{R} \\
\Delta \mathbf{R}
\end{array}\right)=\widetilde{\mathbf{F}}
$$

where,

$$
\begin{aligned}
& \widetilde{\mathbf{K}}=\mathbf{K}-\omega^{2} \mathbf{M}-j \omega \mathbf{C} \\
& \mathbf{K}^{*}=\lambda^{2} \mathbf{M}+\lambda(\mathbf{C}-j 2 \omega \mathbf{M})+\widetilde{\mathbf{K}}
\end{aligned}
$$

Introducing following variant $\mathbf{E}(\mathbf{R})$,

$$
\mathbf{E}(\mathbf{R})=\widetilde{\mathbf{F}}-\widetilde{\mathbf{K}} \mathbf{R}
$$

Combining equations from 5.13 to 5.15 results in,

$$
\left[\begin{array}{cc}
\mathbf{M} & \mathbf{0} \\
\mathbf{0} & \mathbf{M}
\end{array}\right]\{\dot{\mathbf{v}}\}+\left[\begin{array}{cc}
\mathbf{0} & -\mathbf{M} \\
\mathbf{K}_{\mathbf{E}} & \mathbf{C}_{\mathbf{E}}
\end{array}\right]\{\mathbf{v}\}=\left[\begin{array}{l}
0 \\
0
\end{array}\right]
$$


where, $\mathbf{v}=\left(\begin{array}{ll}\boldsymbol{\Delta} \mathbf{R} & \boldsymbol{\Delta} \dot{\mathbf{R}}\end{array}\right)^{T}$, and

$$
\begin{aligned}
& \mathbf{K}_{\mathbf{E}}=\widetilde{\mathbf{K}}-\mathbf{J} \\
& \mathbf{C}_{\mathbf{E}}=\mathbf{C}-j 2 \omega \mathbf{M} \\
& \mathbf{J}=\frac{\partial \mathbf{E}}{\partial \mathbf{R}}
\end{aligned}
$$

where, $\mathbf{J}$ is the Jacobian matrix obtained during the iteration to solve the steady state responses. The Jacobian related to the converged solutions from the iteration will be used for the stability analysis.

More details about the derivation for stability analysis in the frequency domain are included in Appendix B. To this point, the system stability can be determined by the eigenvalue of equation 5.16 using similar procedure applied to the force coefficients method(part 4.5).

\subsection{Predictor-Corrector Procedure}

As discussed in the section 5.1.1, the main effort for the calculation using harmonic balance method is the iteration between the dynamic behaviors and reacting forces of the nonlinear parts. Therefore, the efficiency of the iteration dominates the overall computational speed of this method. There are several facts that determine the iteration efficiency, including initial guess and iteration method. The discussion about the efficiency between different iteration methods is available in other books, such as [70] and [71]. The focus of this part focuses on the effects of initial guess.

Similar to any other iteration method, the distance between initial guesses and final solutions has great influence on the converging speed. Too far way from the final solution usually causes longer iteration times to convergence, or the solution might be diverging. To ensure the initial guess converging to the final solution effectively and efficiently, the idea of Homotopy[113] is adopted in current work. 


\subsubsection{Homotopy}

The equation 5.7, which is required to be solved iteratively, can be generalized as[114],

$$
\mathbf{g}(\mathbf{R}):=\mathbf{f}(\mathbf{R}, \boldsymbol{\Omega})=\mathbf{0}
$$

The main process of the iteration is the involvement of a sequence of approximated solutions,

$$
\mathbf{R}_{(0)}, \mathbf{R}_{(1)}, \ldots, \mathbf{R}_{(m)}, \ldots, \mathbf{R}_{(M)}
$$

where, $\mathbf{R}_{(0)}$ is initial guess, and $\mathbf{R}_{(m)}$ is the approximated solution toward the final solution. Once $\mathbf{R}_{(m)}$ satisfies an error tolerance (small residual) requirement,

$$
\left\|\mathbf{g}\left(\mathbf{R}_{m}\right)\right\|<\epsilon
$$

the iteration will be terminated.

In Homotopy[113, 114], a chain of functions is also introduced. The chain starts with simple functions that are easy to solve, and ends with the target function like 5.18. The transition of functions between the simple and the complicated target function is shown as follows,

$$
\begin{aligned}
& \mathbf{f}^{[0]}(\mathbf{R}):=\widetilde{\mathbf{g}}(\mathbf{R})=\mathbf{0} \\
& \mathbf{f}^{[1]}(\mathbf{R})=\mathbf{0} \\
& \ldots \\
& \mathbf{f}^{[j]}(\mathbf{R})=\mathbf{0} \\
& \ldots \\
& \mathbf{f}^{[Z-1]}(\mathbf{R})=\mathbf{0} \\
& \mathbf{f}^{[Z]}(\mathbf{R}):=\mathbf{g}(\mathbf{R})=\mathbf{0}
\end{aligned}
$$

For each step, the solution of the previous function serves as the initial guess for the following function. Thus, if the difference between $\mathbf{f}^{[j]}$ and $\mathbf{f}^{[j+1]}$ is small, then their corresponding 
solutions, $\mathbf{R}^{[j]}$ and $\mathbf{R}^{[j+1]}$, should be close to each other.

With the above assumption, for any $j$ th intermediate function, the iteration from $\mathbf{R}_{(0)}$ to $\mathbf{R}_{(M)}$ should be fast. Note that the initial guess and converged solution of any $j$ th function have following relation with $\mathbf{R}^{[j]}$ and $\mathbf{R}^{[j+1]}$,

$$
\begin{aligned}
& \mathbf{R}_{(0)}:=\mathbf{R}^{[j]} \\
& \mathbf{R}_{(M)} \approx \mathbf{R}^{[j+1]}
\end{aligned}
$$

The problem now is how to construct the simplest and the intermediate functions during the process of Homotopy. Since the main difference between the simple and complex one comes from one or multiple parameters, say $\eta$. Then, $\eta$ can be described as the range between the simplest and the most complicated condition, such as $[0,1] . \eta=0$ represents the simplest function $\mathbf{f}^{[0]}(\mathbf{R})$ at the beginning of the sequence, and $\eta=1$ represents the target function $\mathbf{f}^{[Z]}(\mathbf{R})$. Any value of $\eta$ within $(0,1)$ represents the transition functions of the chain. The mathematical model of these functions can be described as,

$$
\begin{aligned}
\mathbf{f}^{H}(\mathbf{R}, \eta) & =0 \\
\mathbf{f}^{H}(\mathbf{R}, 0) & =\widetilde{\mathbf{g}}(\mathbf{R}) \\
\mathbf{f}^{H}(\mathbf{R}, 1) & =\mathbf{g}(\mathbf{R})
\end{aligned}
$$

For the nonlinear rotor-bearing systems with SFDs, $\eta$ can depict parameters including unbalance and external loads. For systems with excessive unbalance forces, SFDs responses with high nonlinearity, which introduces difficulties for traditional iteration methods. Using the idea of Homotopy, the system with small unbalance force serves as the simplest system. With small unbalance forces, the SFD operates within the linear range around the damper center. The solution from this linear system serves as the initial guess for the next system with slightly larger unbalance forces. This process continues until the value of the excessive unbalance is applied.

Another scenario having severe nonlinear performances exists in SFDs without centering 
spring, where the gravity of the rotating systems plays an important role to affect their behaviors. For these kinds of systems, the system without the consideration of gravity is considered as the plain system. The systems with gradually increased gravity form the sequence cases to obtain the solution with full load. If the increase of gravity is small enough, the solutions of these systems should be close to each other and the convergence is guaranteed.

For both cases, the excessive unbalance and external loads, there are infinite systems between $\eta=0$ and $\eta=1$, theoretically. However, the application of homotopy is of interest only in the solutions of the final equation. Any intermediate solutions for $\eta$ within $(0,1)$ are abandoned. To avoid the infinite functions of the sequence, the current work applies the procedure of predictors and correctors to determine the number of cases between the simplest and the most complicated systems.

\subsubsection{Predictors}

The previous part discussed the method to obtain the steady state solutions at a fixed operating speed. If the prediction of a speed range is required, additional methods such as predictors and correctors are necessary to improve the computational efficiency. This section concentrates on the predictor procedures. There are two main classes of the predictors[114, 115], the ordinary derivative method and polynomial extrapolation method.

The governing equation for the derivative method is obtained by the differential of equation 5.18

$$
d \mathbf{f}=\frac{\partial \mathbf{f}}{\partial \mathbf{R}} d R+\frac{\partial \mathbf{f}}{\partial \Omega} d \Omega=\mathbf{0}
$$

then, there is,

$$
\frac{d \mathbf{R}}{d \Omega}=-\left(\frac{\partial \mathbf{f}}{\partial \mathbf{R}}\right)^{-1} \frac{\partial \mathbf{f}}{\partial \Omega}
$$

Integration of the above equation results in the prediction of the solution for next speed case. For this derivative method, only one speed case at the beginning of the speed range is required. Thus, the process of homotopy will only be applied once, which saves computation 
time somehow. However, the problem for this method is that the inverse of $\frac{\partial \mathbf{f}}{\partial \mathbf{R}}$ might not exist for every speed case. The derivative would be singular at some turning points [116]. To avoid this problem, parameterization method such as arc-length continuity method can be used $[117,118]$.

For the polynomial extrapolation method, one or more speed cases might be needed using homotopy process, depending on the order of chosen polynomial. For the first order polynomial, it is trivial prediction, which means $\mathbf{R}_{0}\left(\Omega_{j+1}\right)=\mathbf{R}_{M}\left(\Omega_{j}\right)$. If the polynomial has the order of one, then two previous speed cases are required to give the prediction for next speed cases. The prediction is given by,

$$
\mathbf{R}_{0}\left(\Omega_{j+1}\right):=\mathbf{R}_{M}\left(\Omega_{j}\right)+\delta_{j}\left(\mathbf{R}_{M}\left(\Omega_{j}\right)-\mathbf{R}_{M}\left(\Omega_{j-1}\right), \Omega_{j}-\Omega_{j-1}\right)
$$

Where $\delta_{j}$ is a given step length. Higher order polynomial is also possible for the extrapolation[119], however, lower-order predictors cost less in the long run[3]. The current work adopted the secant method for the prediction.

\subsubsection{Correctors}

The solutions given by predictors are usually not the right one satisfying the prescribed error requirement for convergence. Therefore, correctors are necessary to correct the predictors towards the solution on path. The dot line in figure 5.1 shows the path of correction from the predictor. The path from the predictor to the converged solution for speed $\Omega_{j+1}$ would be different dependent on the method of parameterizations[119, 3]. However, no matter what parameterization method is chosen, the process of correction needs iteration procedure such as Newton-Raphson. At each iteration step, the next corrector can be obtained with current corrector as,

$$
\begin{aligned}
& \mathbf{R}_{z+1}=\mathbf{R}_{z}+\Delta \mathbf{R} \\
& \Omega_{z+1}=\Omega_{z}+\Delta \Omega
\end{aligned}
$$


Then, the problem is to calculate $\Delta \mathbf{R}$ and $\Delta \Omega$ at every iteration step. One way to obtain the increment is to use the tangent predictor and search the variation along the orthogonal direction of the tangent, which can be described by scalar product as follows,

$$
\left(\mathbf{R}_{z}\left(\Omega_{z}\right)-\mathbf{R}_{M}\left(\Omega_{j}\right)\right)^{T} \Delta \mathbf{R}+\left(\Omega_{z}-\Omega_{j}\right) \Delta \Omega=0
$$

Together with first order partial derivative shown in equation 5.24, $\Delta \mathbf{R}$ and $\Delta \Omega$ are solved for next correction step.

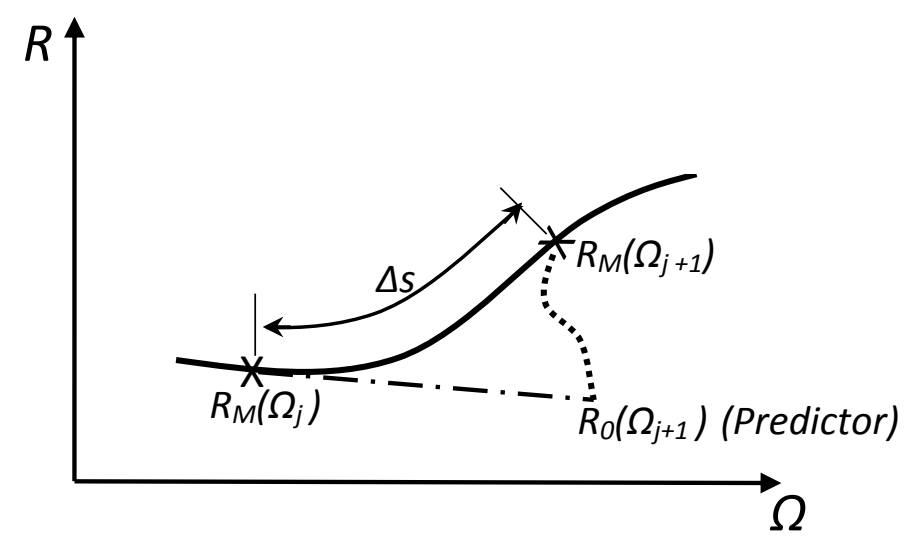

Figure 5.1: Iteration of a corrector [3]

The overall process of the improved harmonic balance method with predictors and correctors is illustrated by the scheme in figure 5.2.

\subsection{Application to a Flexible Rotor-Bearing System}

This section applies the improved harmonic balance method to the flexible rotor-bearing as described in chapter 4 . The finite element beam model and geometry parameters of the rotor can be found in figure 4.6 and table 4.4. The geometry and operating conditions of the damper is listed in the following table 5.1. The damper is assumed to be open-end with $\pi$-film cavitation. Thus, the same model of SFD developed in chapter 4 will be applied. The operating speed of the rotor is from 2,000 rpm to 10,000 rpm. The effects of excessive 


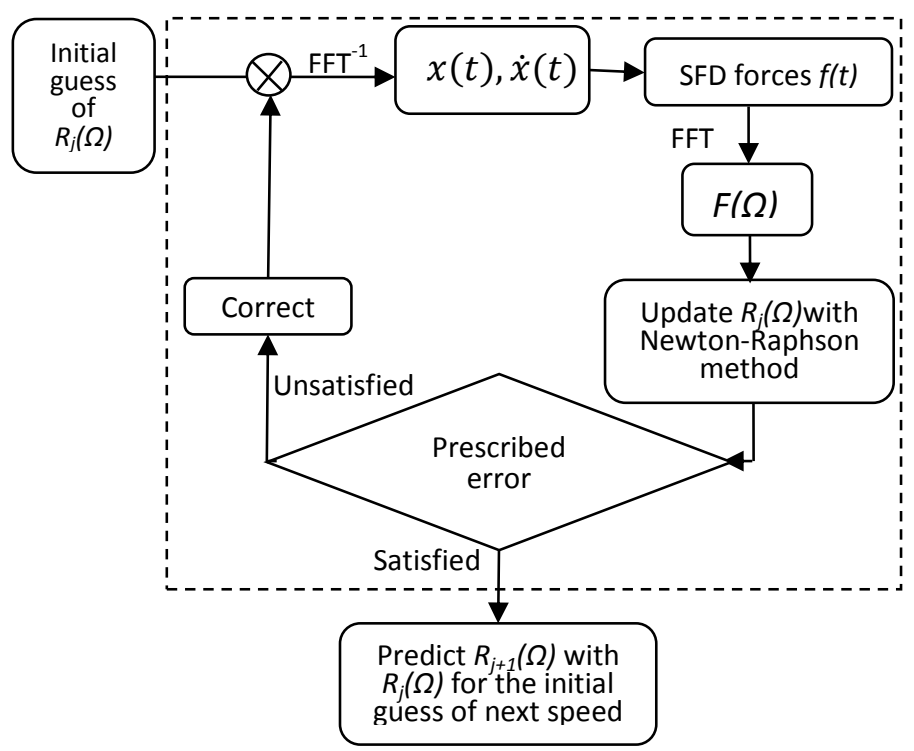

Figure 5.2: The Scheme of Improved Harmonic Balance Method

unbalance, unidirectional loads and gyroscopic on the forced responses of the system are investigated. The results from force coefficients method and the improved harmonic balance method are compared for both unbalance responses and stability.

Table 5.1: Geometries and Operating Conditions of the Damper for HBM

\begin{tabular}{l|l}
\hline Journal Diameter $(D)$ & 3 in \\
\hline Axial Length $(L)$ & 1 in \\
\hline Radial Clearance $(C)$ & $6.67 / 1000$ in \\
\hline Lubricant & ISO VG 32 \\
\hline
\end{tabular}

\subsubsection{Responses with Varying Unbalance}

The responses of the damper to unbalance applied at the locations of three disks are evaluated. The magnitude and phase angle of the unbalance are applied to one disk only for each calculation at 0.6 oz-in and 0 degrees, respectively. As depicted in figure 5.3, the critical speeds and their amplitudes for the three cases are quite similar, with the largest amplitude for unbalance on Disk 2 and the least on Disk 1. This indicates that the squeeze film damper is more sensitive to unbalance on Disk 2 than the similar unbalance on other disks, which is the same observation as in chapter 4 . With larger unbalance magnitude (0.7oz-in), the 


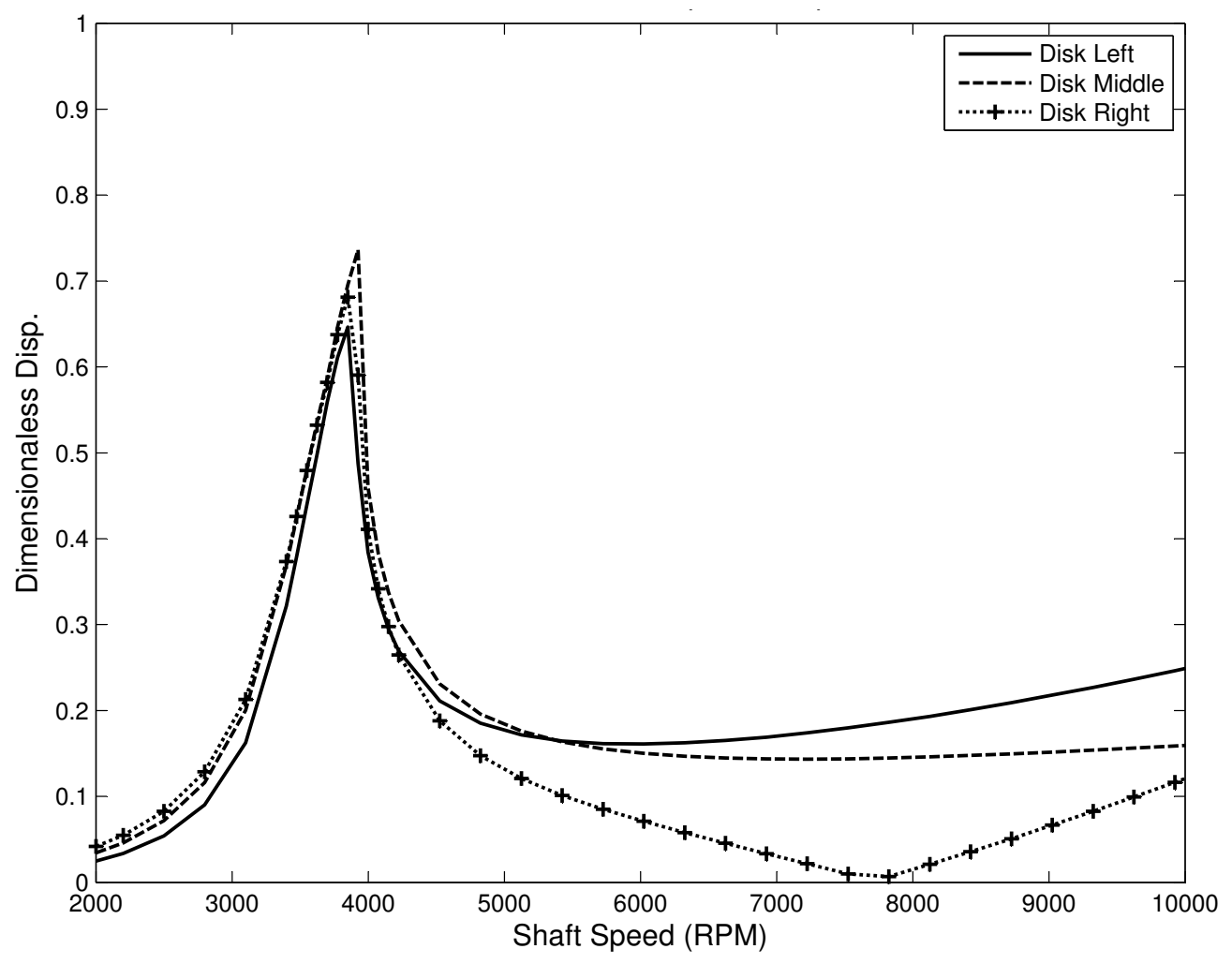

Figure 5.3: Response of Damper, $\mathrm{U}=0.6$ oz-in

response of the damper starts to jump, as shown by figures from 5.4 to 5.6. The jump happens between 3,890 and 4,200 rpm for these cases. The speed difference between start-up and coast-down is the largest of $180 \mathrm{rpm}$ when the unbalance is located on Disk 2, as compared to $110 \mathrm{rpm}$ for unbalance in Disk 3 and $97 \mathrm{rpm}$ for unbalance on Disk 1. The corresponding hysteresis area in Fig. 6 is the largest. This implies that the squeeze film damper is the most responsive to unbalance in Disk 2. Note that the responses shown from figure 5.4 to 5.6 are excited by unbalance on disks only, without considering other external forces. Thus, the reason for the observed hard and soft spring effects of nonlinear jump phenomena above is the presence of excessive unbalance $(0.7 \mathrm{oz}$-in is about the magnitude of $233 \mathrm{~W} / \mathrm{N}$, about 58 times as large as API requirement of $4 \mathrm{~W} / \mathrm{N})$. Figure 5.7 displays the FFT of response at 3,990 rpm during start-up for unbalance on Disk 2. The super-harmonics of the $3 \mathrm{x}$ component starts to appear, together with the synchronous component. 


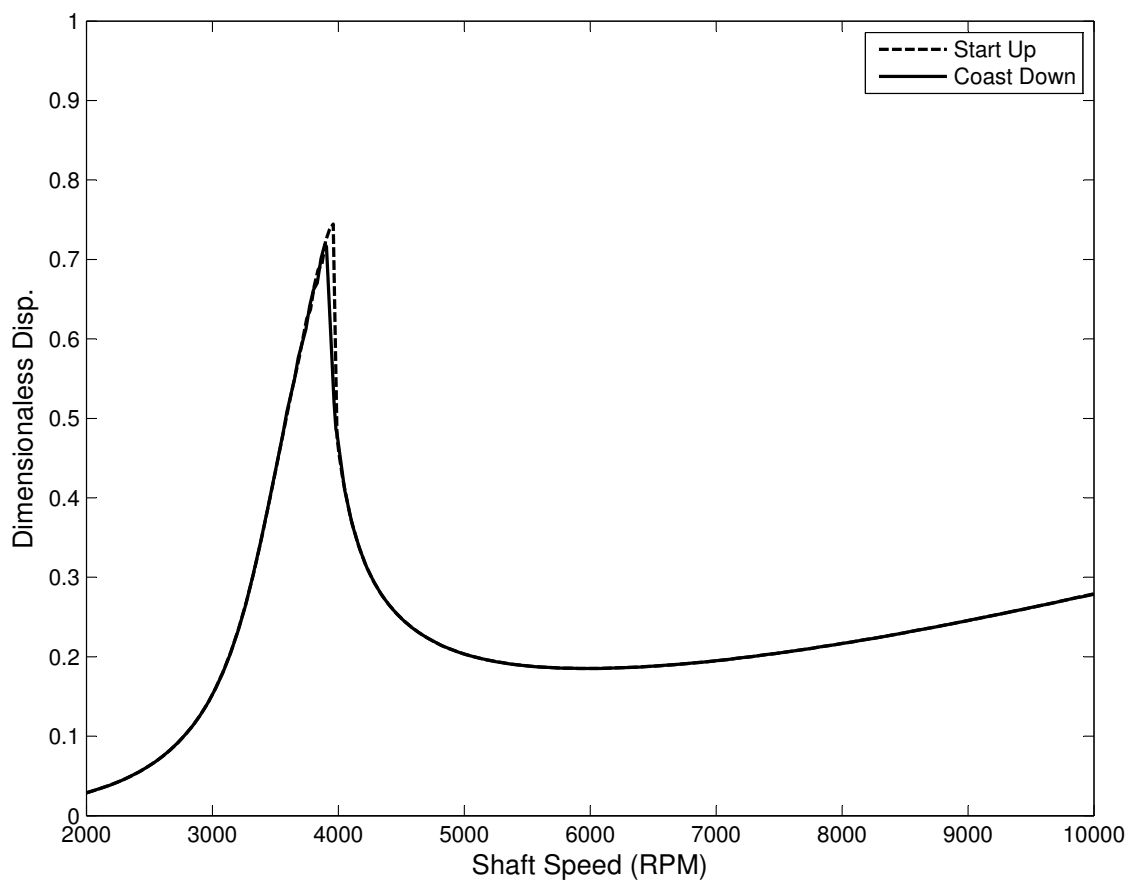

Figure 5.4: Response of Damper, $\mathrm{U}=0.7$ oz-in on Disk 1

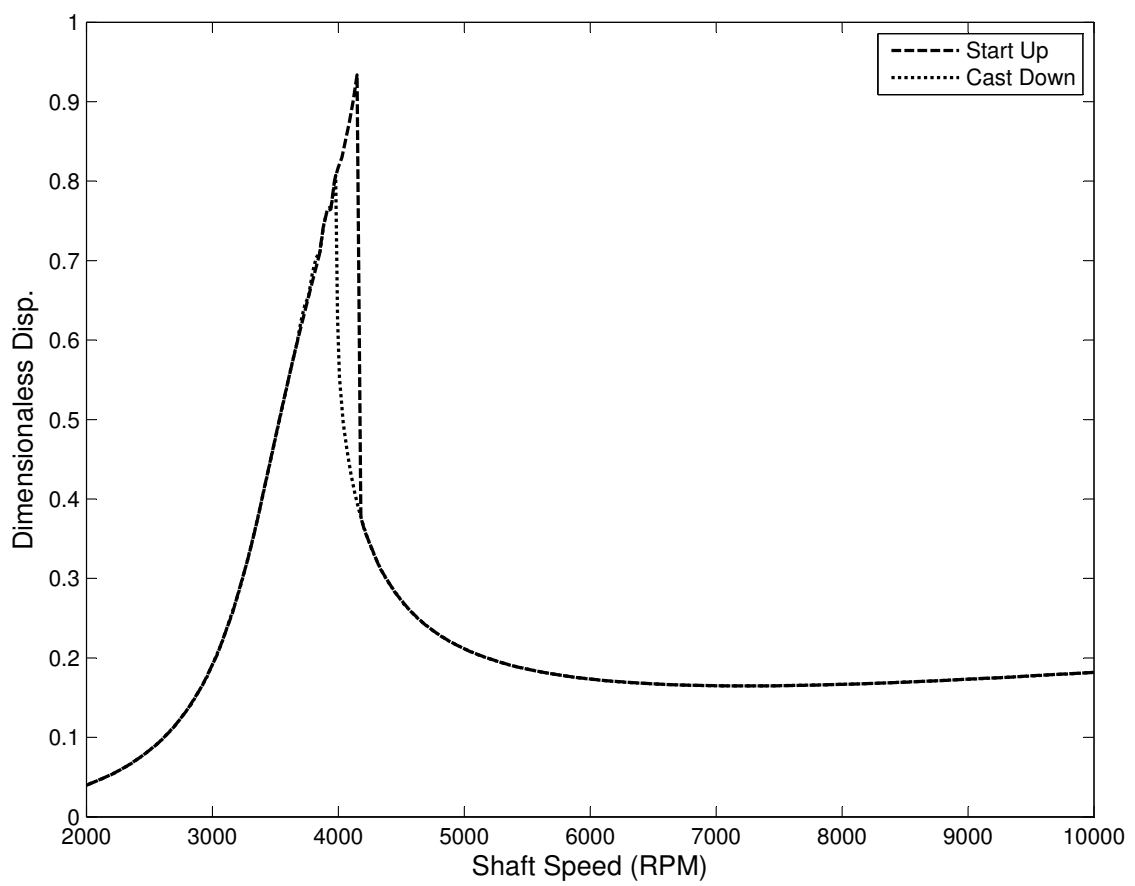

Figure 5.5: Response of Damper, $\mathrm{U}=0.7$ oz-in on Disk 2 
5.3 Application to a Flexible Rotor-Bearing System

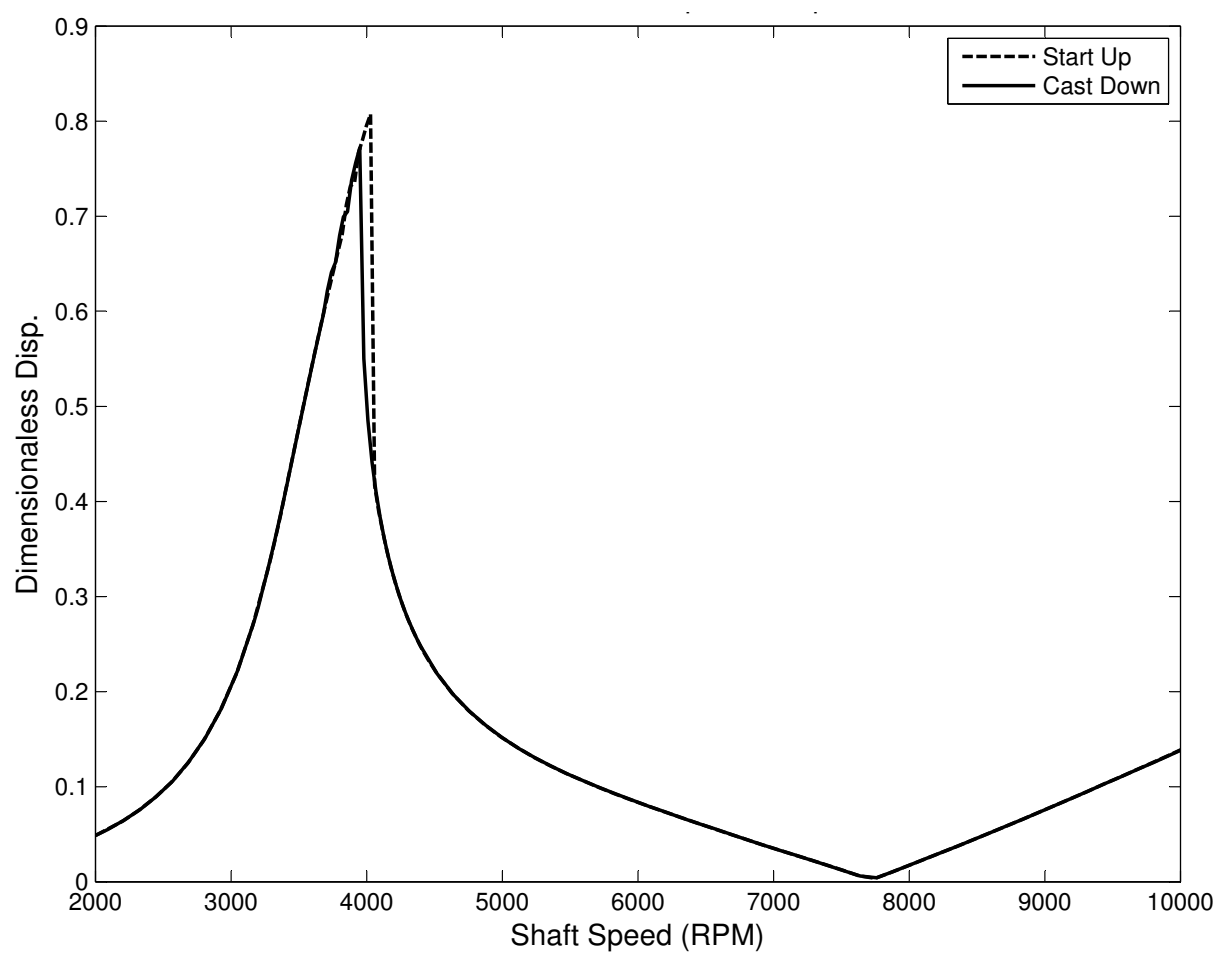

Figure 5.6: Response of Damper, $\mathrm{U}=0.7$ oz-in on Disk 3
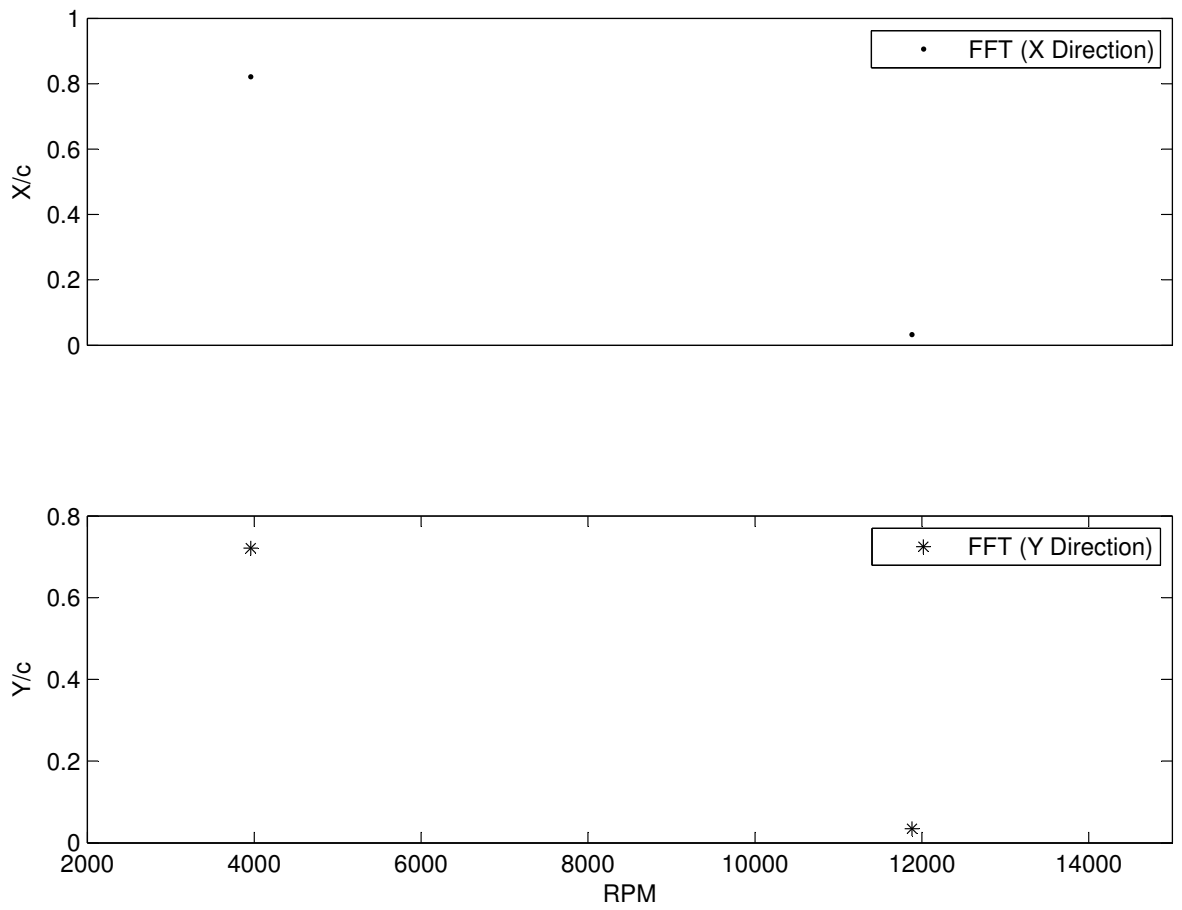

Figure 5.7: FFT of Response at 3990 rpm, with Unbalance on Disk 2 


\subsubsection{Responses with Unidirectional Loads}

The unidirectional loads are applied by including the gravity of both rotor and disk. Compared to previous cases without gravity, the damper operates eccentrically and is more nonlinear. With the same unbalance of 0.6 oz-in on Disk 2, figure 5.8 gives the comparison between responses with and without loads. The maximum amplitude of the response with load at the critical speed is quite similar to the case without load. Despite the fact that the damper vibrates with larger amplitude for speeds far away from the critical speed region, the difference between cases with and without gravity load decreases as the operating speed approaches the first critical speed. This result displays some evidence for the nonlinearity of the squeeze film damper, which can also be found by the nonlinear jump phenomenon shown in figure 5.8. With other conditions unchanged but the load, the damper starts to jump when the unbalance on Disk 2 is 0.6 oz-in, as compared to the 0.7 oz-in for the case without the consideration of gravity. The evaluation of varying unidirectional loads is carried out by

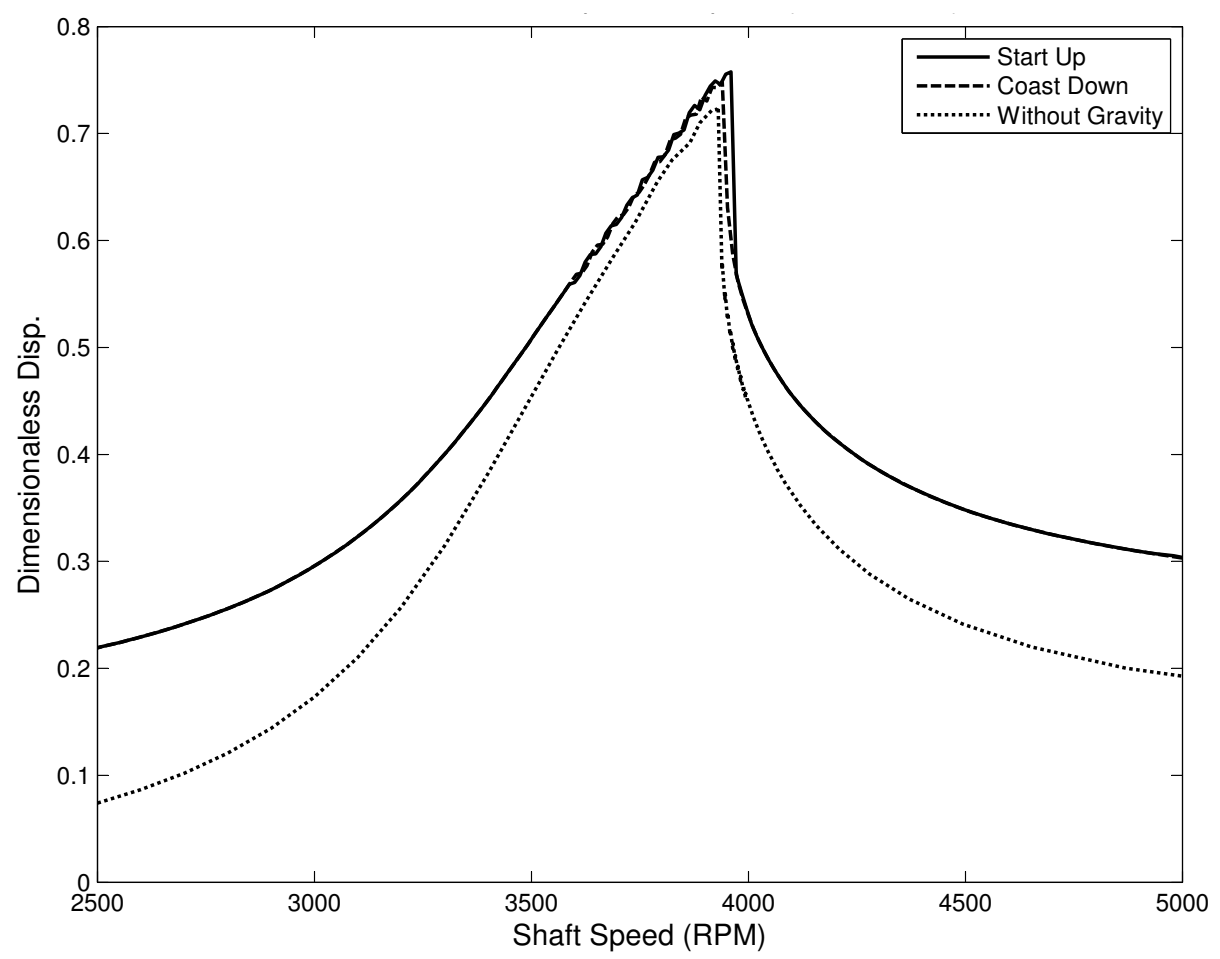

Figure 5.8: Response of Damper with Load, $\mathrm{U}=0.6$ oz-in on Disk 2 
applying an additional force at the location of Disk 3 , since it is the closest to the damper and produces more eccentric operation condition than other disks. The forces are in the same direction as gravity, and their amplitudes change from 1 times the gravity on Disk 3 up to 16 times. With constant unbalance having magnitude of 0.4 oz-in on Disk 2 and phase angle of 0 degree, figure 5.9 displays the responses to various loads.

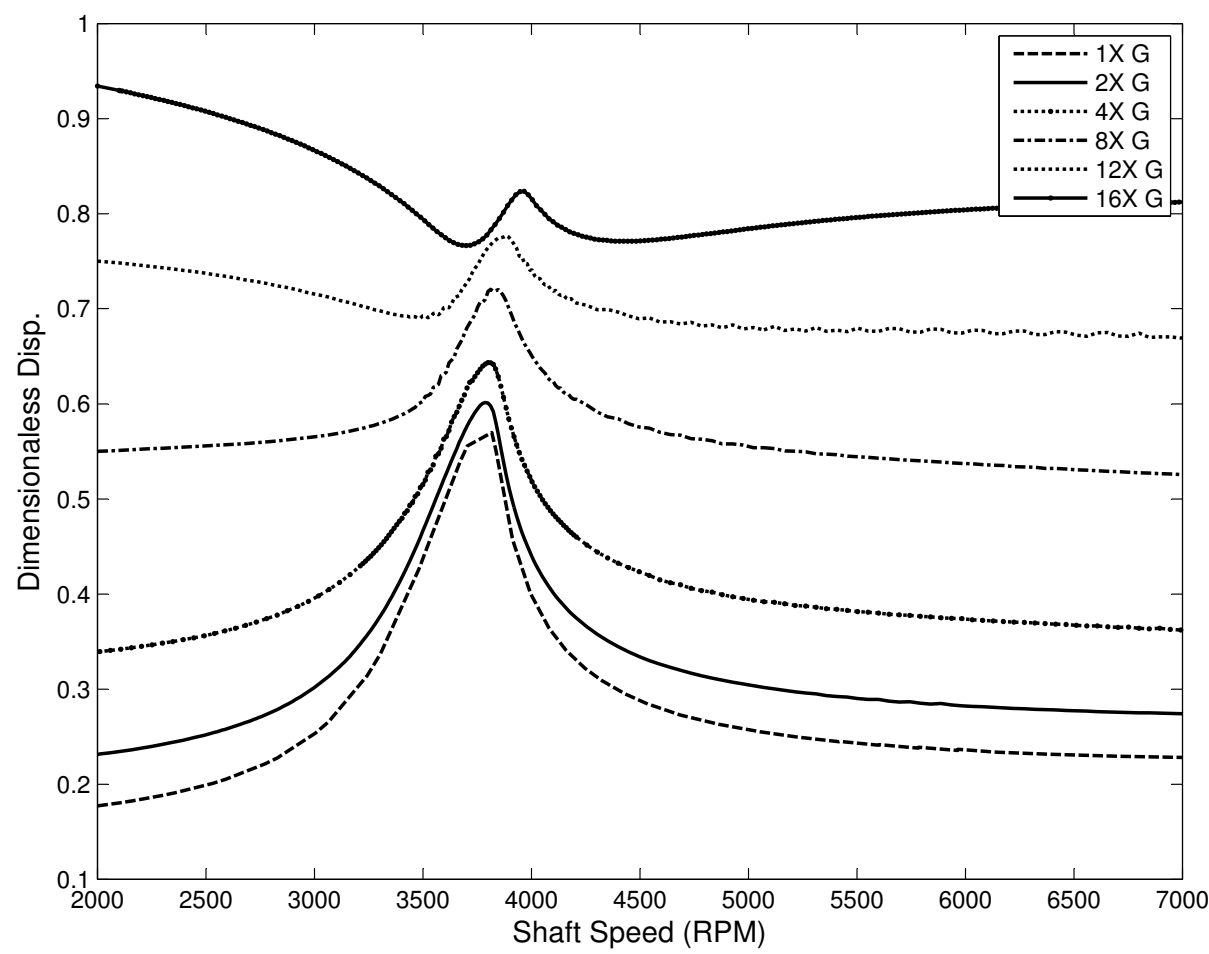

Figure 5.9: Response of Damper, with Varying Loads on Disk 3

As expected, higher loads push the damper to a more eccentric region. The first critical speeds, shown in figure 5.9, shift to the right as loads increase, which comes from operating condition having high eccentricity. The amplitude of the critical speed decreases, in contrast to the displacement of speeds around $2000 \mathrm{rpm}$; for the case of $16 \mathrm{x} \mathrm{G}$, it does not have the highest amplitude, and is smaller than that of low speed. Within low operating speed region, the dynamic forces are not large enough to balance the external static loads on the soft centering spring supported damper. The increasing speed, although it lifts the journal upward somehow, cannot pull the damper out of high eccentric region that prevents responses with large amplitude. 
This fact of increasing nonlinearity with loads can also be observed from the shape change of damper orbits as well. Figure 5.10 gives the orbits at 7,000 rpm, for loads up to $16 \mathrm{x}$ G. The shapes of the orbits change from an off-centered circle to an ellipse with shrinking amplitude, indicating that the damper produces more asymmetry with high damper operating eccentricity. The non-circular shape also implies that multiple harmonics are present for large static loads, which can be confirmed by the FFT in Figure 5.11. All of these non-circular responses with multiple harmonics cannot be obtained using the force coefficients method in the previous chapter, since the force coefficients method is based on the assumption of synchronous Centered Circular Orbit.

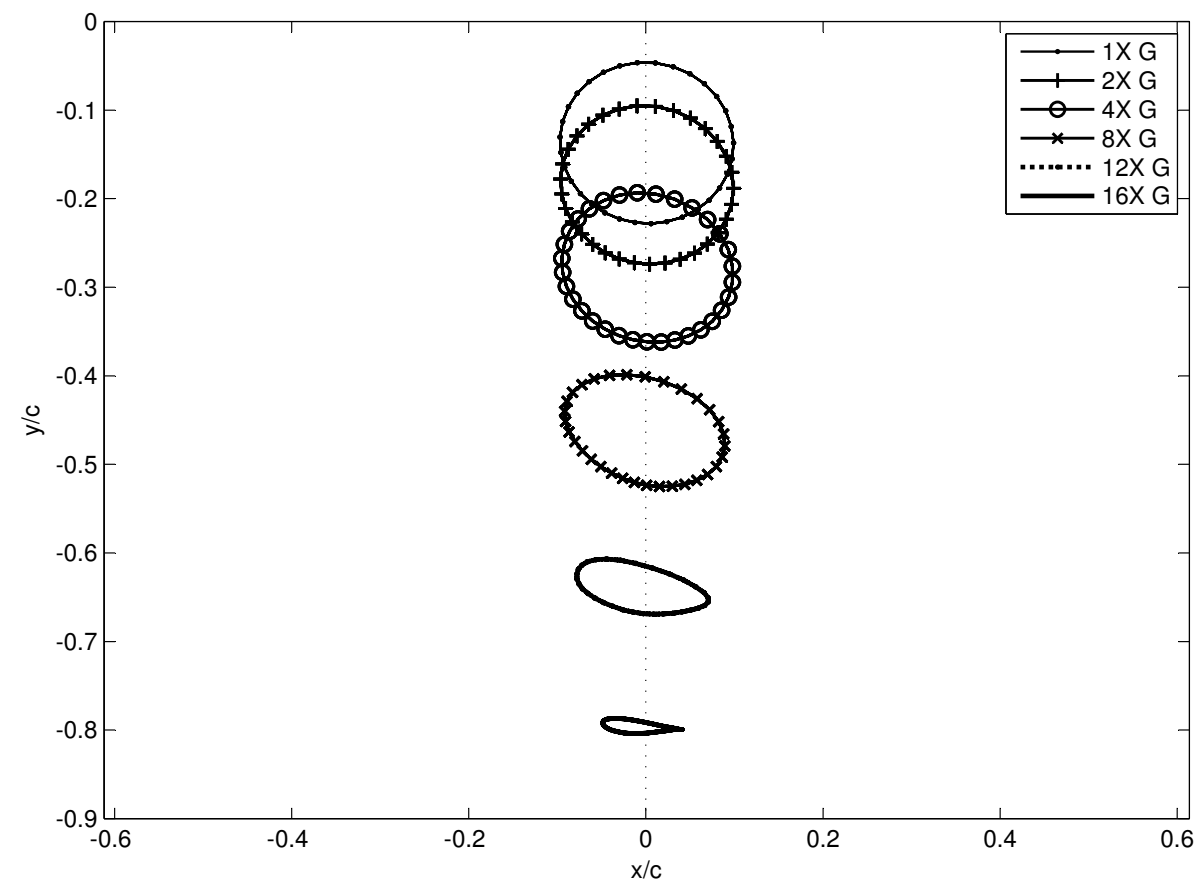

Figure 5.10: Orbits at 7000 rpm, with Varying Loads on Disk 3

The zeroth frequency component in the y direction, which is shown by the offsets of the orbits in figure 5.10, is not included in FFT. The appearance of a zeroth component in the $\mathrm{x}$ direction, which is also indicated by figure 5.10, implies that the center of orbit becomes eccentric not only in the y direction as the static load increases up to 4 times the weight of Disk 3. Although the $1 \mathrm{x}$ component is dominant for all the tested cases, it's amplitude 

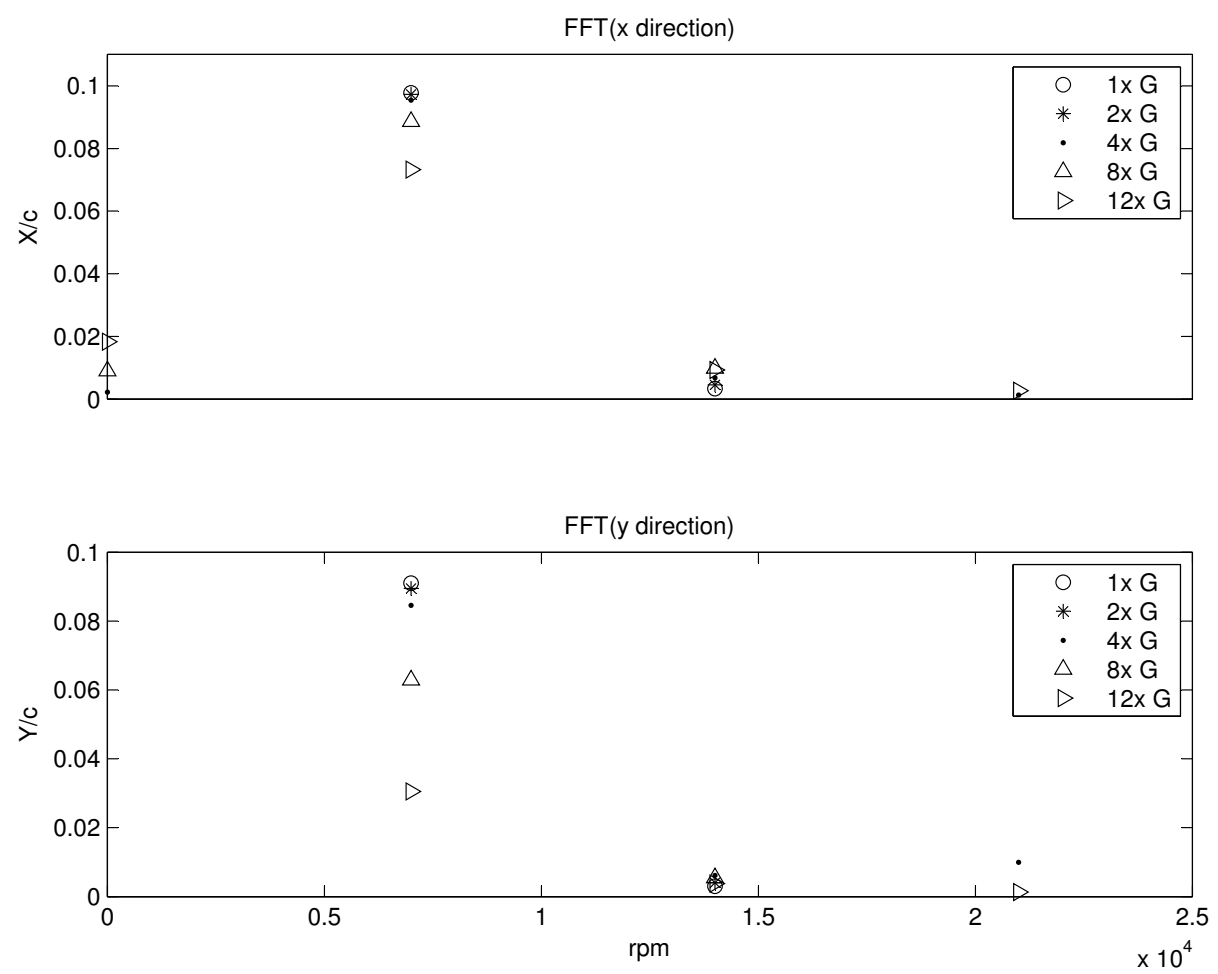

Figure 5.11: FFT of orbits (7000 rpm) with Varying Load

decreases with growing unidirectional static loads. Meanwhile, the increasing static loads lead to an excitation of the $2 \mathrm{x}$ and $3 \mathrm{x}$ harmonics within the unbalance response results and produce non-circular orbits as shown above.

\subsubsection{Responses with Varying Stiffness of Centering Spring}

To evaluate damper responses with a varying stiffness of centering spring, the unbalance is kept on Disk 2 with fixed magnitude of 0.4 oz-in and phase of 0 degree. Both cases with and without the consideration of gravity are examined.

\section{Varying spring stiffness WITHOUT gravity}

Figure 5.12 displays the responses for spring stiffness ranging from $100 \mathrm{lbf} /$ in to 10,000 lbf/in without gravity. As the centering spring stiffness increases, the critical speed does not increase all up to the highest value as found in the linear system models. The first critical speed 
decreases as the centering spring stiffness increases within the region from 100 to 2,500 lbf/in.

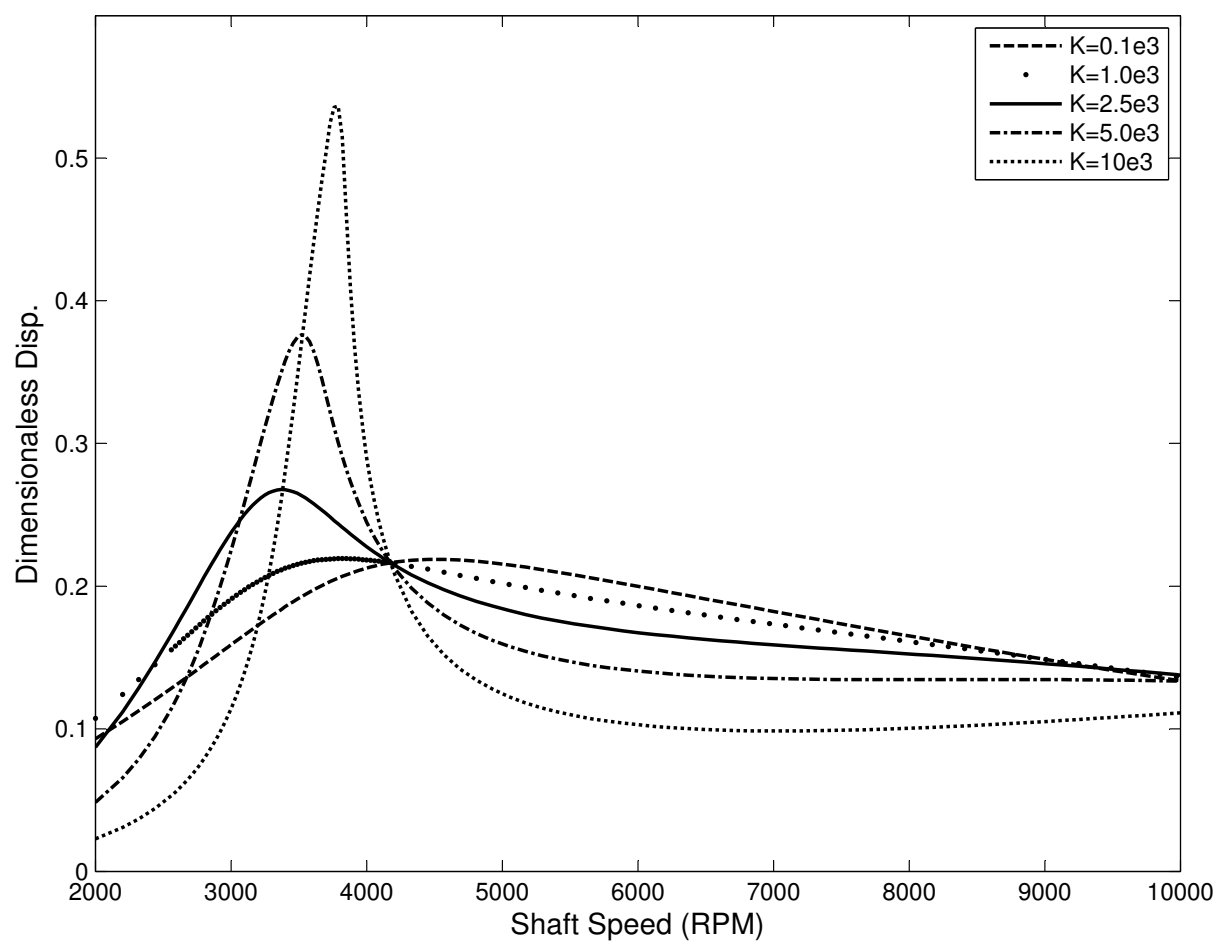

Figure 5.12: Response without Gravity

As given in figure 5.12, the amplification factors are smaller than 2.5 for the soft centering spring region. This indicates that the squeeze film damper acts effectively without the restriction from the stiff centering spring and produces a well-damped system. For speeds above than 4,100 rpm, the damper with low centering spring stiffness, on the other hand, operates with larger amplitudes. This observation is quite similar to results with base excited flexible rotor vibrations for frequencies beyond the first critical.

\section{Varying spring stiffness WITH gravity}

With the addition of gravity, Figure 5.13 gives the response with centering spring stiffness range from 1,500 to $10,000 \mathrm{lbf} / \mathrm{in}$. As the centering spring stiffness decreases, the damper operates with even larger eccentricity, similar to the cases with increasing unidirectional loads. Contrasting this result to the cases in Figure 5.9, the shapes of the responses in Figure 5.13 
change with centering spring stiffness. The critical speed around 3,700 rpm for stiff spring supports is decaying with decreasing stiffness; when $\mathrm{K}$ is less than 2,500 lbf/in. The vibration crosses the region smoothly without the presence of a peak.

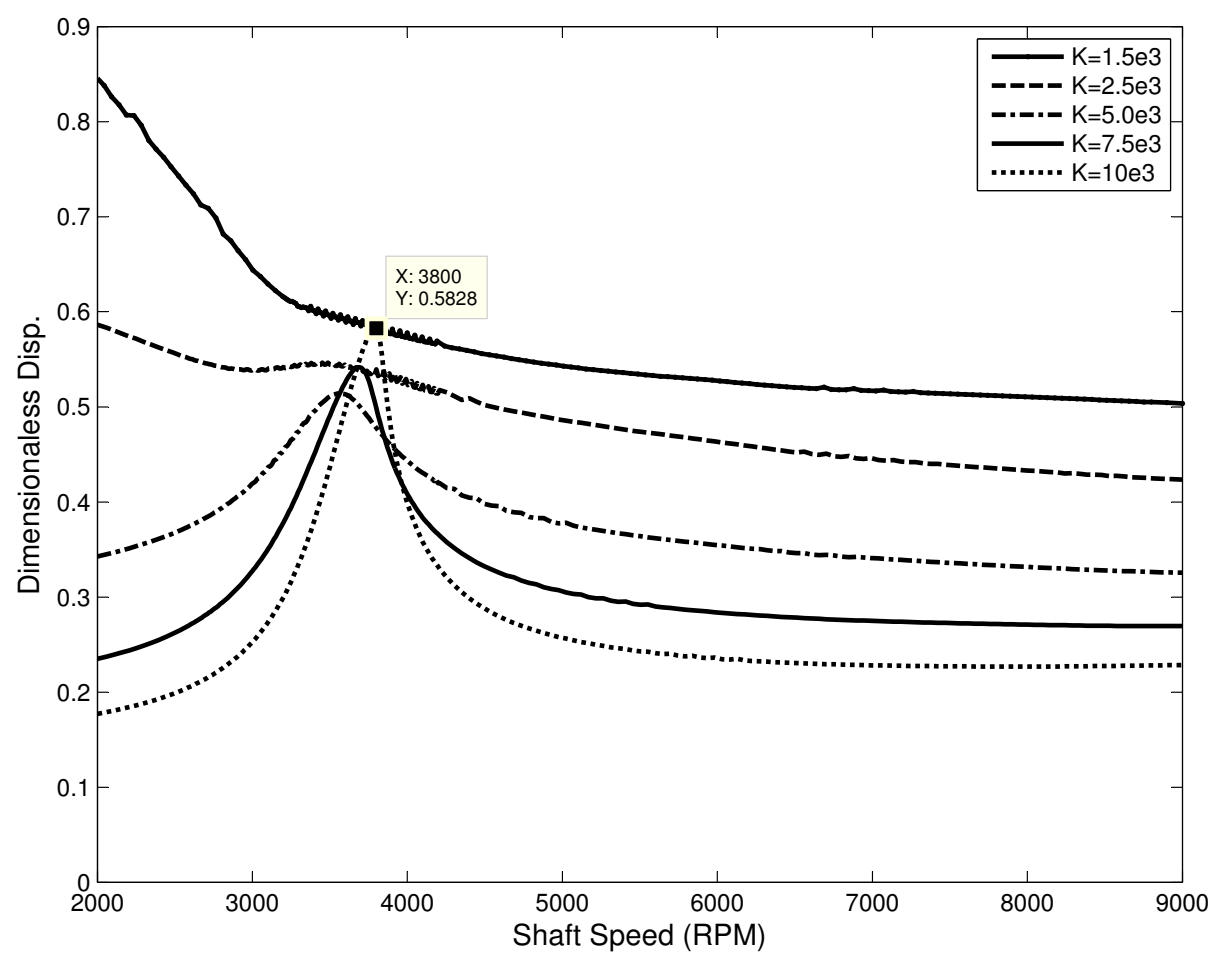

Figure 5.13: Response with Gravity

Comparing Figure 5.13 to the results in Figure 5.12, the responses with a hard centering spring have a slightly higher first critical speed and amplitude; e.g., the critical speed for $\mathrm{K}=10,000 \mathrm{lbf} / \mathrm{in}$ is $3,800 \mathrm{rpm}$ with dimensionless amplitude of 0.58 , which is larger than the results at 3,790 rpm for the cases without gravity. For both cases, the system is shown to be well-damped with soft centering springs by the evidence of the disappearance of critical speed for K less than 2,500 lbf/in.

For the rotor operating with speeds below 3,000 rpm, the gravity effect dominates the total response for soft centering spring supports and results in high eccentricity operation, which excites super harmonics as expected. Figure 5.14 depicts a flat orbit at 2,000 rpm with spring stiffness of 1,500 lbf/in. The orbit has small amplitude, indicating rather large hydrodynamic forces acting on the journal. A larger displacement in $\mathrm{x}$ direction than $\mathrm{y}$ shows 
that the damper generates higher forces along y direction, exhibiting the high asymmetry of the damper. Also, the FFT of the y direction response contains multi-harmonics including the 0th (not shown in Figure 5.15),1x, 2x and 3x, as compared to the $\mathrm{x}$ direction response which has the 0th and 1x component only.

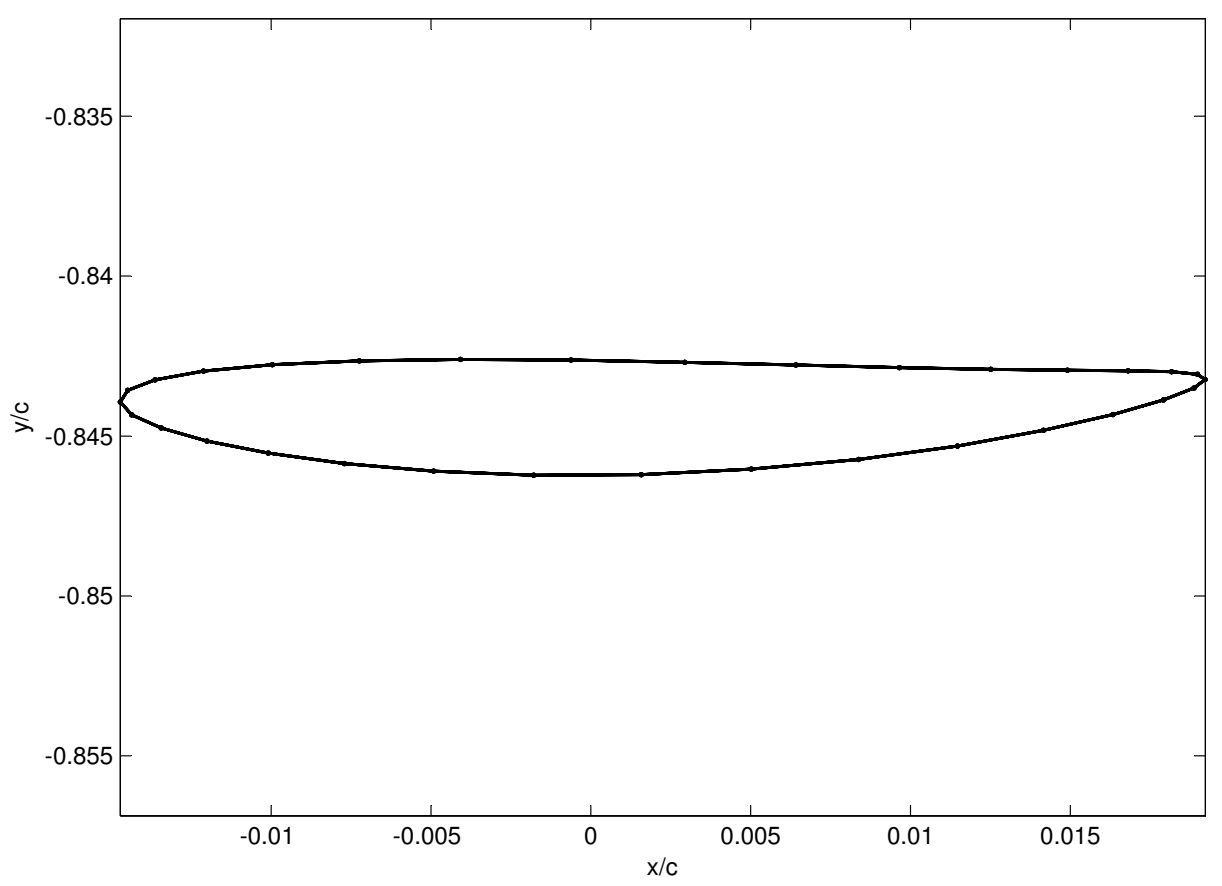

Figure 5.14: Orbit at $2000 \mathrm{rpm}, \mathrm{K}=1500 \mathrm{lbf} / \mathrm{in}$

\subsubsection{Responses with Effects of Gyroscopics}

Responses with different gyroscopic terms in the equation of motion are evaluated by changing the polar inertia of each disk. An unbalance of 0.4 oz-in is retained on Disk 2 and the gravity effect is not included. The centering spring stiffness is kept the same as 2,500 lbf/in for all the evaluations. Figures from 5.15 to 5.18 display responses of varying polar inertia from $1 \mathrm{x}$ to $16 \mathrm{x}$ of nominal value for each disk.

For the variation of inertia of Disk 1, the smallest inertia results in a maximum response at the damper for speeds less than 3,000 rpm. This order reverses above that speed with 16x disk inertia producing the largest amplitude before all the responses gathering together 

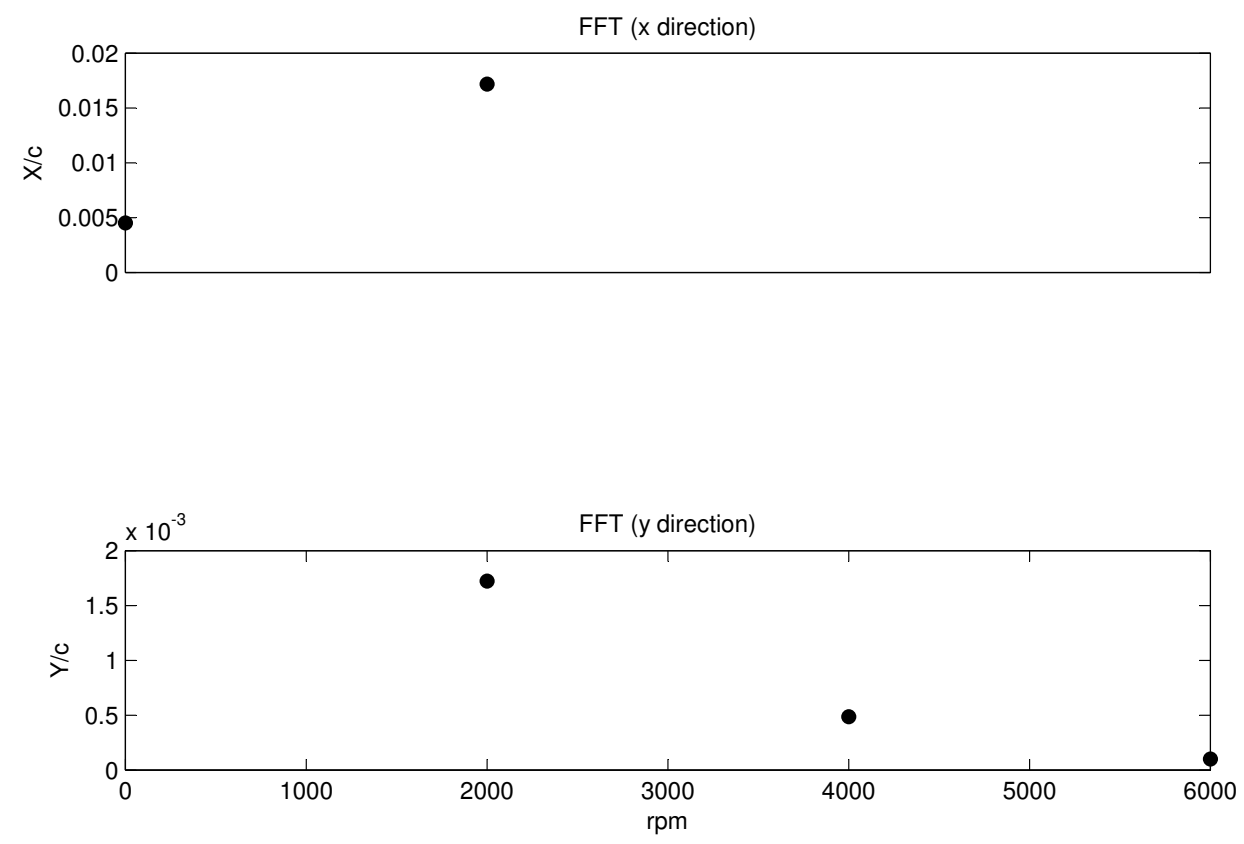

Figure 5.15: FFT of Response at 2000 rpm, K=1500 lbf/in

around 7,000 rpm. By increasing the disk polar inertia, the first critical speed shifts to the right with rising amplitude. One of the reasons of the shift is the hardening effects of the gyroscopic terms on the first forward mode of the system. In addition, due to the stiff ball bearing at left end, the conical mode of the system is more likely to be excited by Disk 1 than the other two, as it locates furthest from the damper.

With the same varying range of the disk polar inertia, Figure 5.17 depicts the damper responses corresponding to the inertia change in Disk 2. The amplitude of all responses is quite similar for operating speeds less than 3,000 rpm. The largest gyroscopic term produces the highest amplitude around the first critical speed and it becomes the lowest for speeds above 4,000 rpm. In contrast to the previous cases, the first critical speed with studied range of polar moment of inertia of Disk 2 are identical, with only a slight increase of the amplitude.

For the variation of the polar moment of inertia of Disk 3, the damper behaves similarly to the second case for speeds below 3,000 rpm, having indistinguishable responses. Contrasting this result to the first case, the increasing polar moment of inertia decreases the first critical 


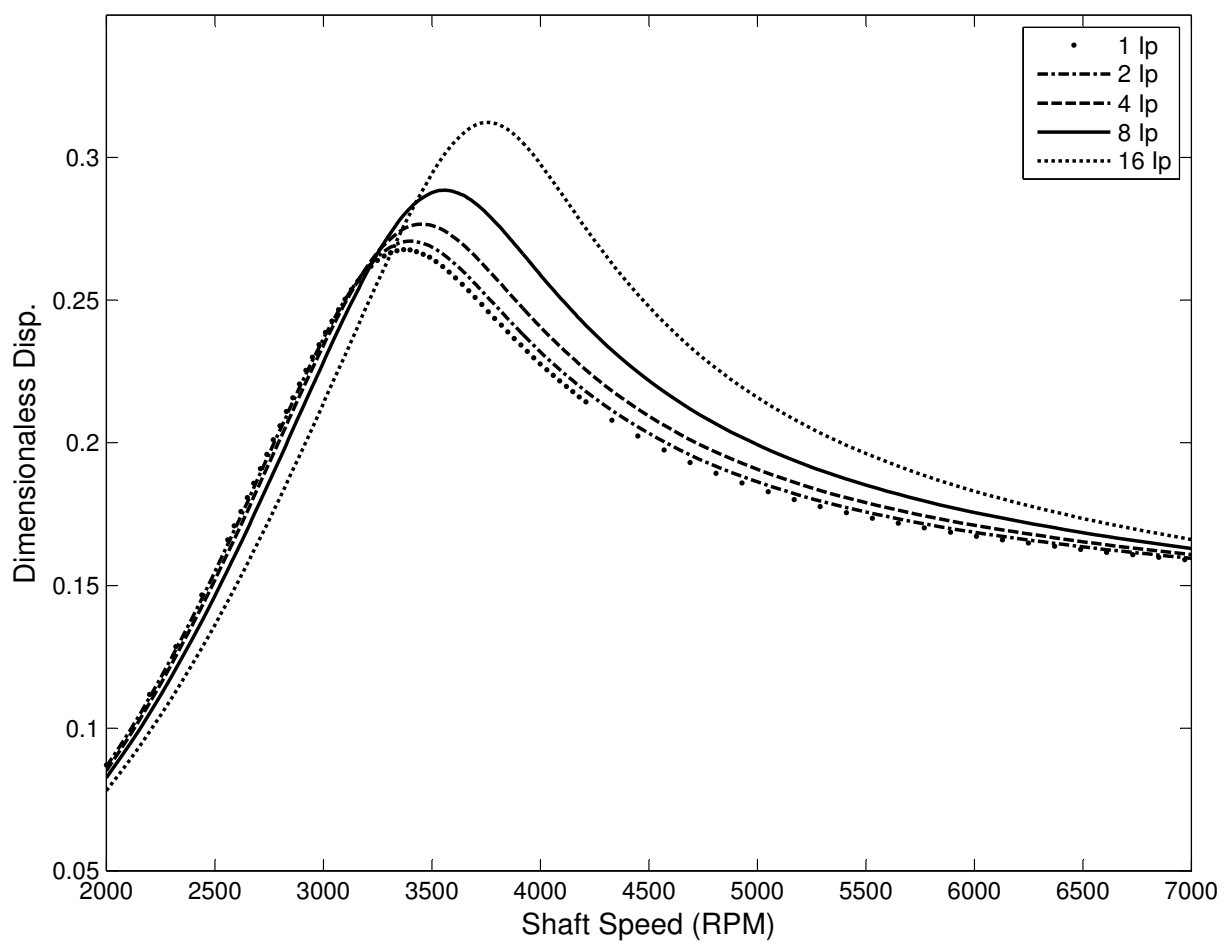

Figure 5.16: Response with Varying Inertia of Disk 1

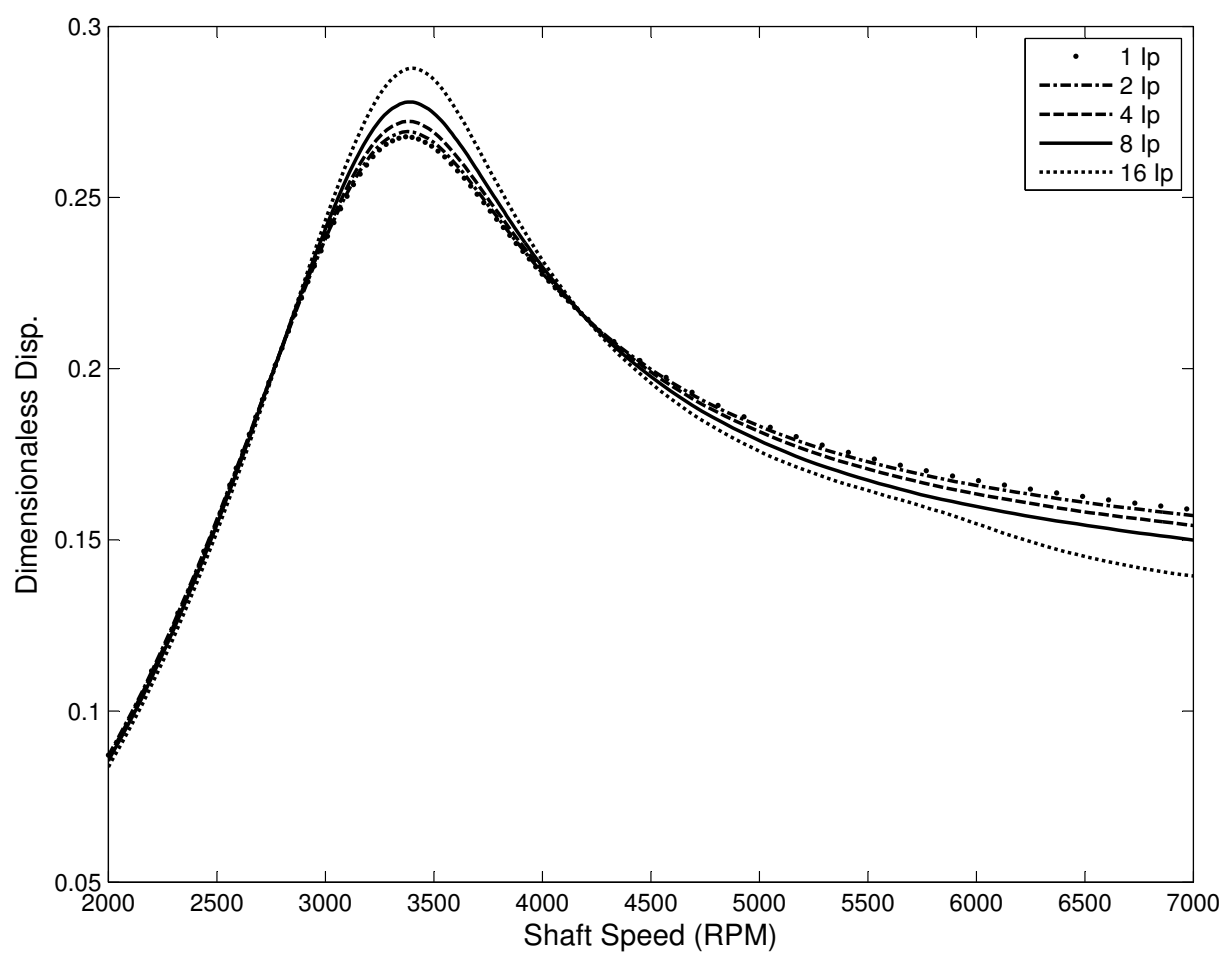

Figure 5.17: Response with Varying Inertia of Disk 2 
speed and its amplitude. Meanwhile, the amplitude with largest polar moment of inertia is helping keep the lowest over the responses of the speed region studied.

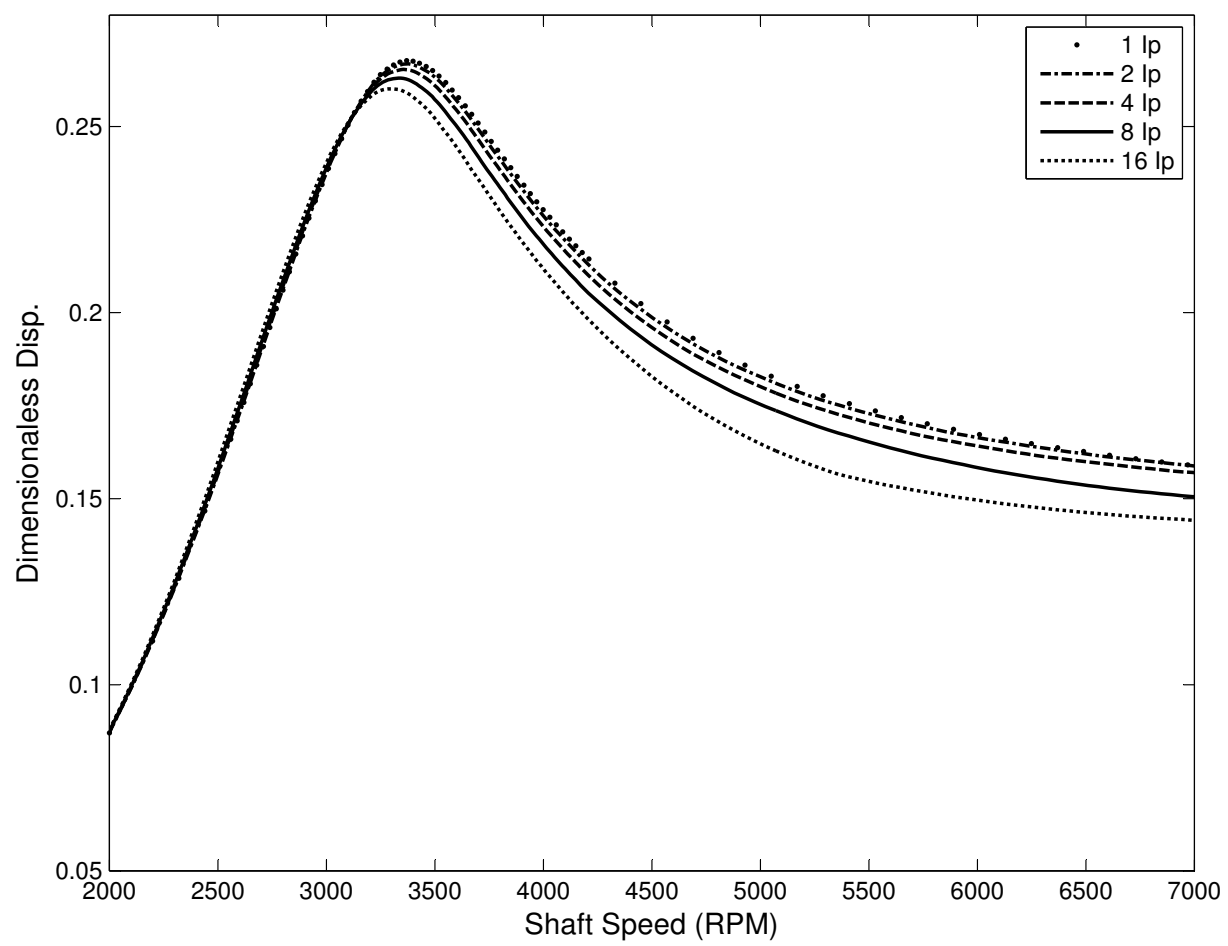

Figure 5.18: Response with Varying Inertia of Disk 3

Comparing all three cases, one can find that, the variance of the responses with the changing polar moment of inertia is different in each of three different cases. Case 1 gives more than a 5\% difference of response between smallest and largest inertia around the critical speed, larger than that of the second case; the varying polar moment of inertia of Disk 3 gives the smallest.

\subsubsection{Comparison with Force Coefficient Method}

The case for comparison with force coefficient method has unbalance on Disk 2 with magnitude of 0.12 oz-in and phase of $0^{\circ}$. The centering spring has the stiffness of 2,000 lbf/in. The effects of gyroscopics are included, but without consideration of gravity. In other words, the damper is assumed to precess within centered orbits. 
Figure 5.19 shows the comparison of unbalance responses between the improved harmonic balance method and force coefficients method. As shown in the figure, the responses from the two methods are almost the same. Both the amplitude and critical speeds values are the same for the two methods. Although the force coefficient method under-predicts the response compared to that from the harmonic balance method for the speed range from 3000 to 8000 rpm, the overall difference between each is neglectable.

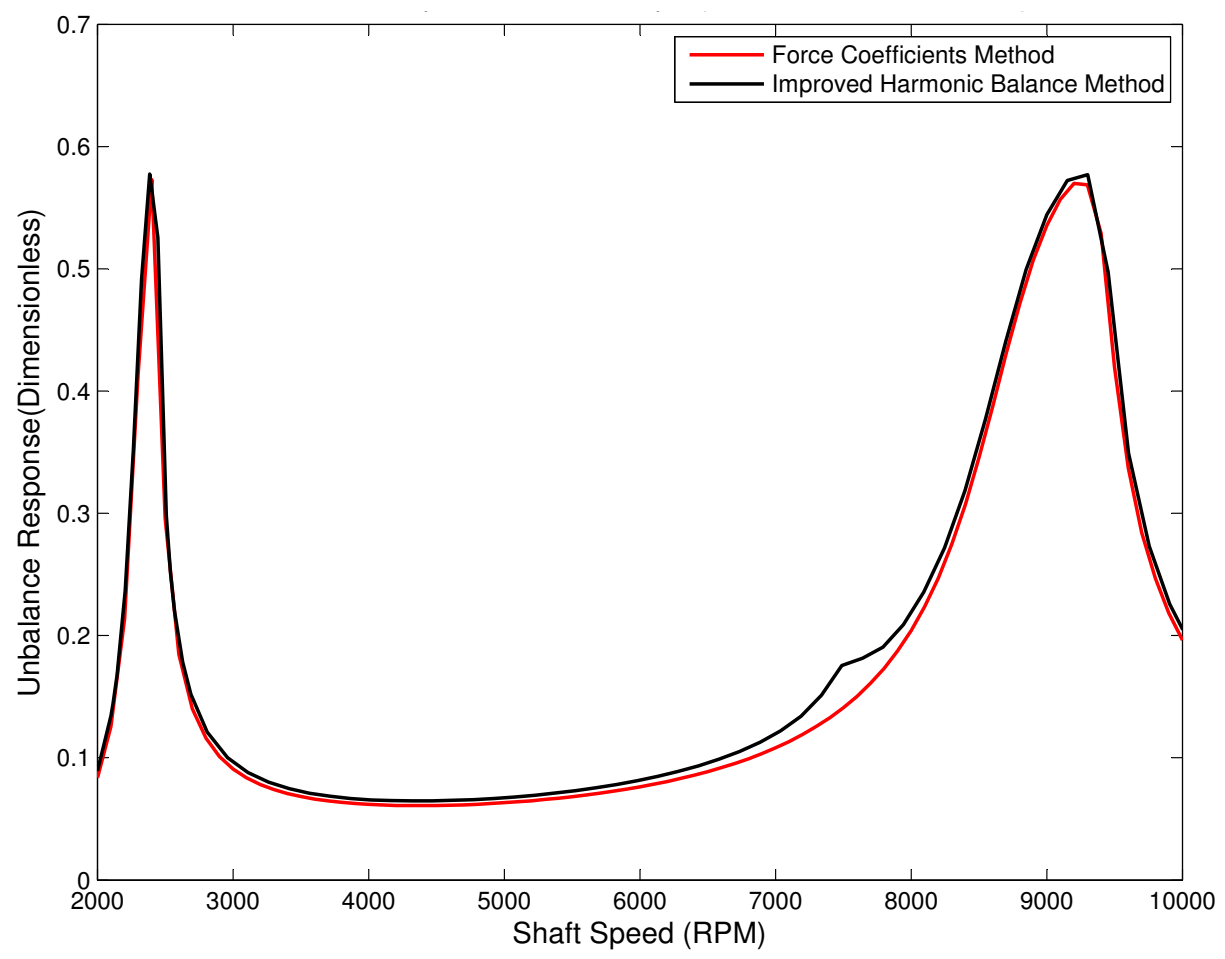

Figure 5.19: Comparison of Responses between HBM and Force Coefficients

The maximum difference happens around the running speed of $7500 \mathrm{rpm}$, where the harmonic balance method gives 3.5\% higher amplitude than the force coefficients method. This happens due to the presence of asymmetric support, which produces an elliptical orbit as given in Figure 5.20. Since one of the basic assumptions of the force coefficients method is the circular centered orbit, therefore, it can not obtain the response accurately. 


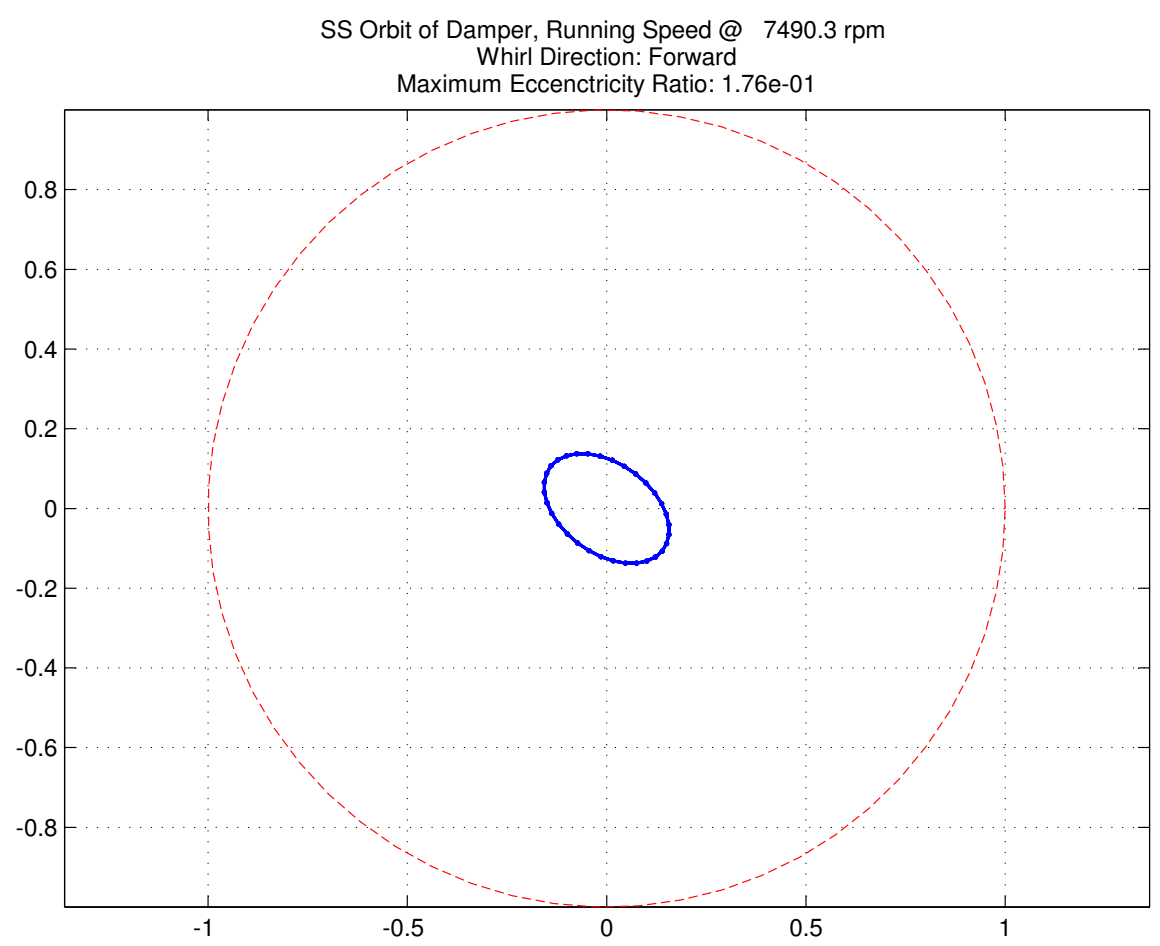

Figure 5.20: Elliptical Orbit at 7490 rpm using HBM

\subsection{Summary}

This chapter has discussed the rotordynamics analysis using direct force method for rotating systems with SFDs. An improved harmonic balance method was developed for the analyses of forced response and stability. The efficiency of traditional harmonic balance method is enhanced by solving the nonlinear parts only during the iteration, and the solutions of the linear parts are obtained without any iterative step. In contrast to other works using harmonic balance method, this chapter developed a new stability analysis method in the frequency domain. Therefore, the computational efficiency is increased by eliminating the requirement for the analysis in the time domain.

The ability of the improved harmonic balance method to analyze highly nonlinear rotating systems is ensured by introducing the idea of homotopy. Excessive dynamic and static loads for SFDs without centering spring can be modeled effectively. For the analysis of operating speed range, the predictor-corrector procedure was introduced to result in fast iteration 
process to gain the solutions of the entire speed range.

The developed direct force method was applied to the flexible rotating system subject to parametric changes, which include the magnitude and location of unbalance forces, unidirectional loads, stiffness of centering spring and gyroscopic effects. The jumped responses were captured by the developed method when excessive unbalance is present. Additionally, the method obtained the non-circular eccentric orbits when static loads are large, which can not be calculated using the force coefficients method. The effects of gyroscopics, which are neglected by other works using harmonic balance method, were found to be important for the forced responses.

The comparison of the responses from both the improved harmonic balance method and force coefficient method shows good agreement between each other. However, the harmonic balance method is able to give more reasonable solutions than the force coefficient method, since it does not have assumptions that constrain it from modelling more complicated responses. 


\section{Chapter 6}

\section{Rotordynamic Analysis of an}

\section{Aero-Engine}

As opposed to ground-based turbines, where fluid film bearings are extensively used, aeroengines are typically supported on rolling element bearings for reduced energy consumption. However, a significant disadvantage to the rolling element bearings is the lack of damping to stabilize the system. To solve this problem, SFDs are introduced to produce additional damping for reliable operations of these machines. This is the primary reason for their widespread use in aero-engines.

This chapter will investigate the rotordynamics analyses of an aero-engine, Rolls-Royce AE 3007, using the methods developed in chapters 4 and 5. The engine has two rotors, a Low-Pressure(LP) rotor and a How-Pressure(HP) rotor. The LP rotor consists of a singlestage fan and 3-stage turbine. The HP rotor has an axial compressor with 14 stages and a turbine with 2 stages. Figure 6.1 shows a cut-away of the AE3007 turbofan. There are three SFDs in the rotating system, two for the LP rotor and one for the HP rotor.

One of the challenges in modeling the aero-engines is the consideration of the SFDs with complicated configurations. Typically, they have end seals, such as piston rings, and supply/discharge conditions like hole and groove. To tackle this challenge, both the force 


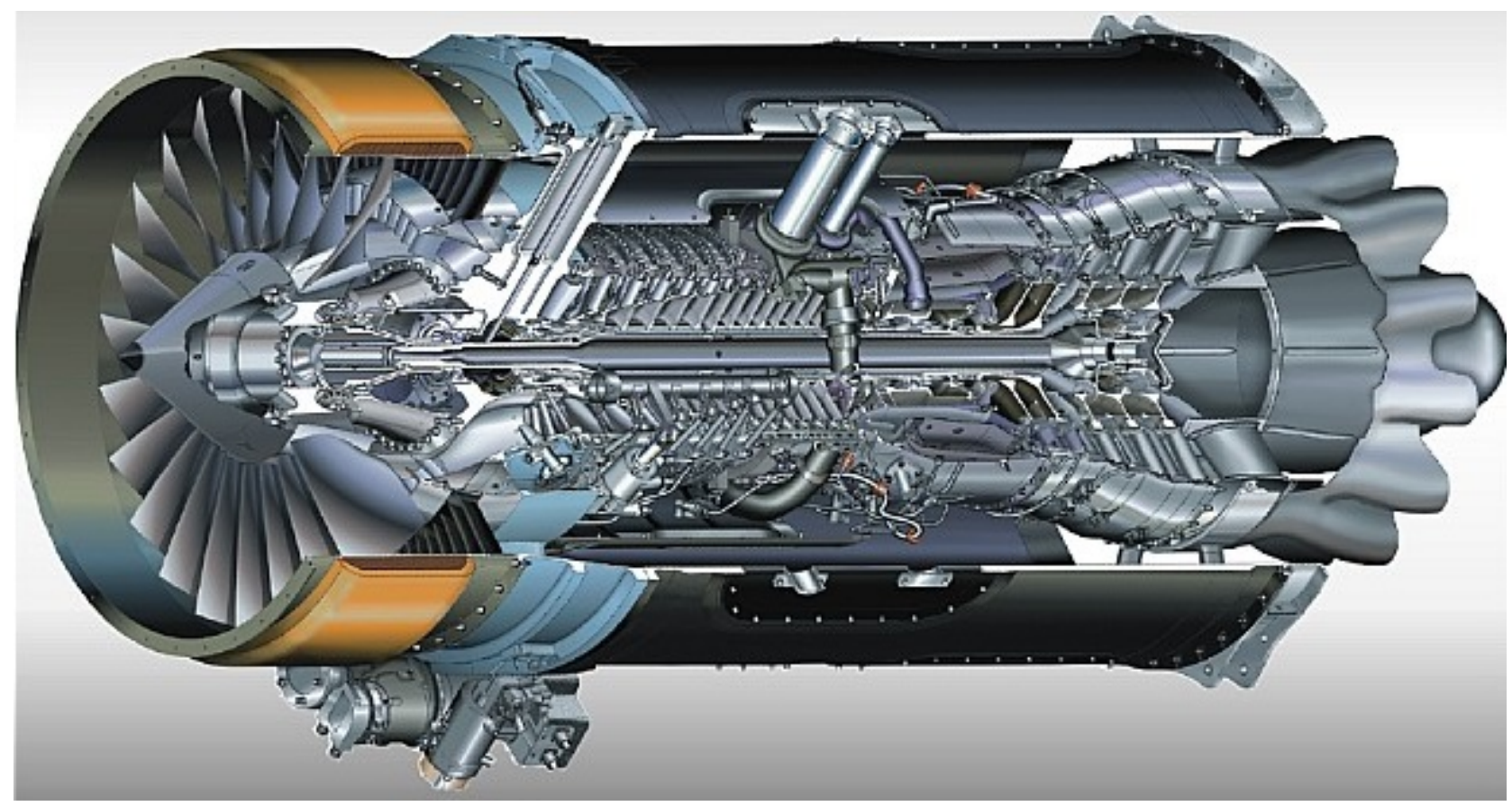

Figure 6.1: Cut-away of Rolls-Royce AE 3007 [4]

coefficients method and the improved harmonic balance method are used in the rotordynamic analysis of the engine. The forced response of the LP rotor will be obtained using the improved harmonic balance method. To demonstrate its computational effectiveness, it is compared to a transient analysis in the time domain. The effects of parametric changes on the responses of the LP rotor are also included in this chapter for the purpose of optimized design. For the HP rotor, a simplified model for SFDs with piston-ring seals is adopted to analyze its unbalance responses and stability using the force coefficients method. With this implified method, the effective parametric studies are realized to give optimized damper design. The stability performance of the HP rotor is documented when subjected to the variation of cross-coupled stiffness at the compressor stages.

Based on the initial design from the force coefficients method, two realistic damper configurations of the HP rotor, piston-ring seal with direct hole supply/discharge and pistonring seal with central groove, are studied using the improved harmonic balance method. The models of sealing and supply/discharge conditions from Chapter 3 are used for the analysis. The responses of the rotor with these two configurations are then compared at the operating 
speed.

\subsection{Forced Responses of the Low Pressure Rotor}

The LP rotor is modeled using 2-D finite element method. The fan disks and turbine stages are approximated as lumped mass with polar and transverse inertia. The conical shape segments of shaft at both fan end and turbine end are approximated by step elements. Figure 6.2 gives both the schematic view and finite element model of the rotor[120]. The rotor has total weight around $180 \mathrm{lbf}$ with maximum running speed about $7500 \mathrm{rpm}$. The rotor is supported by three rolling element bearings. Two squeeze film dampers are located in-series with fan-end (station 4) and turbine-end (station 25) bearing. There are total 100 DOFs of the rotor with 4 additional DOFs of dampers at both ends.

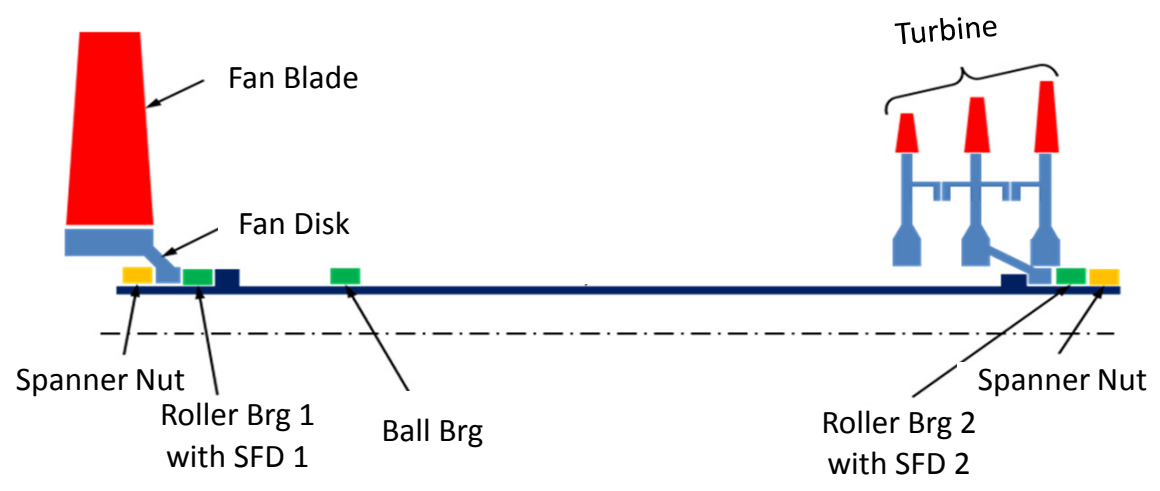

(a) Schematic View of LP Shaft

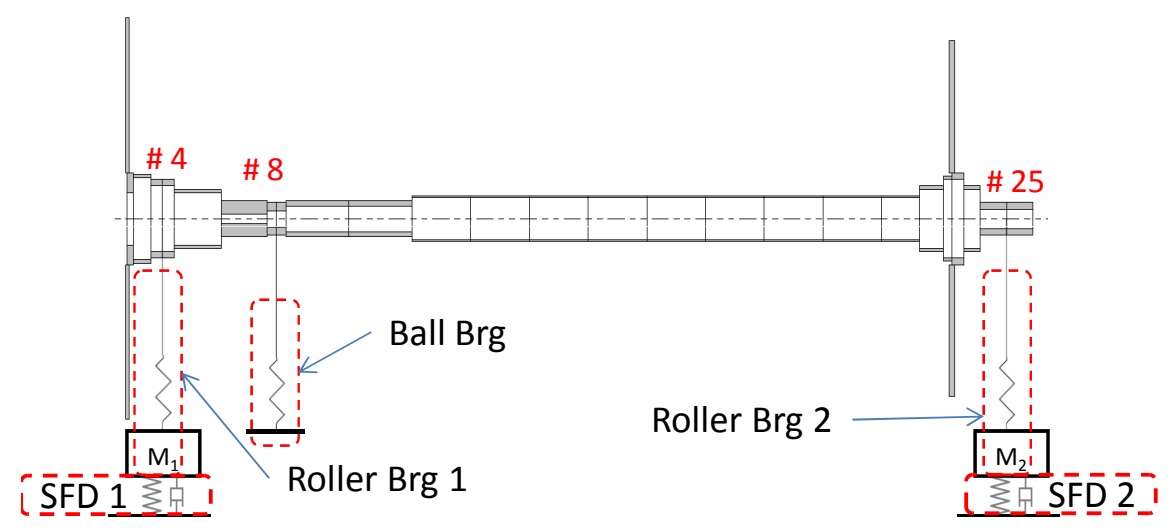

(b) Finite Element Model of LP Shaft

Figure 6.2: Schematic View and FEA Model of LP Rotor 


\subsubsection{Comparison with the Transient Analysis}

The non-dimensional forced responses of the two dampers, the fan-end and turbine-end, at different running speeds from both methods are displayed as Figure 6.3 and 6.4, respectively. All the responses are normalized to the radial clearance of each damper. As depicted, the differences between the results from two different methods are almost negligible, within the range of $1 \%$ of the radial clearance. However, the computational efficiency of HBM is much more preferable than that of Runge-Kutta method, as listed in table 6.1.

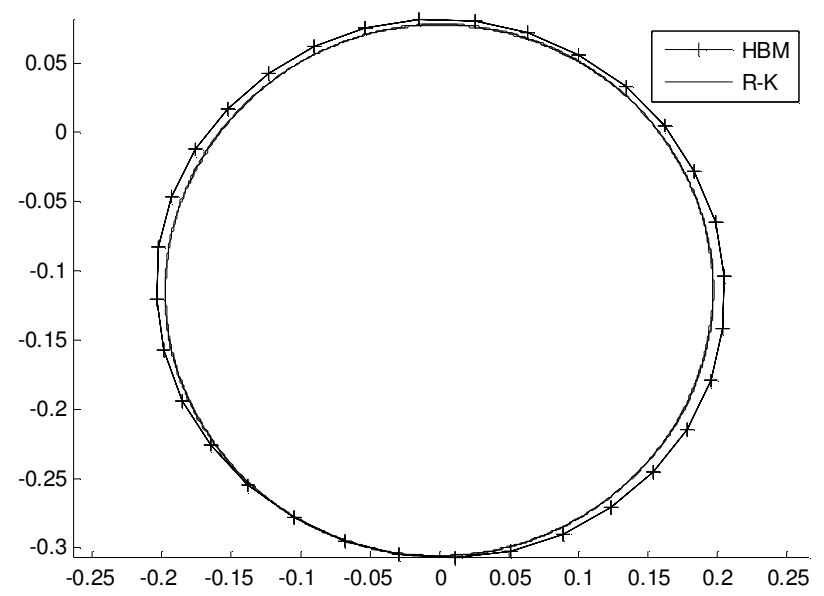

(a) $3500 \mathrm{rpm}$

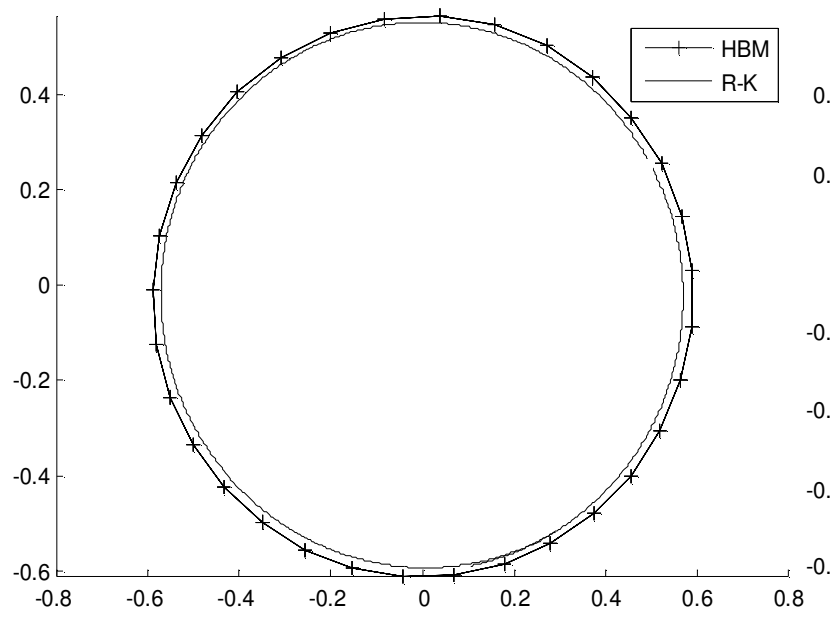

(c) $7000 \mathrm{rpm}$

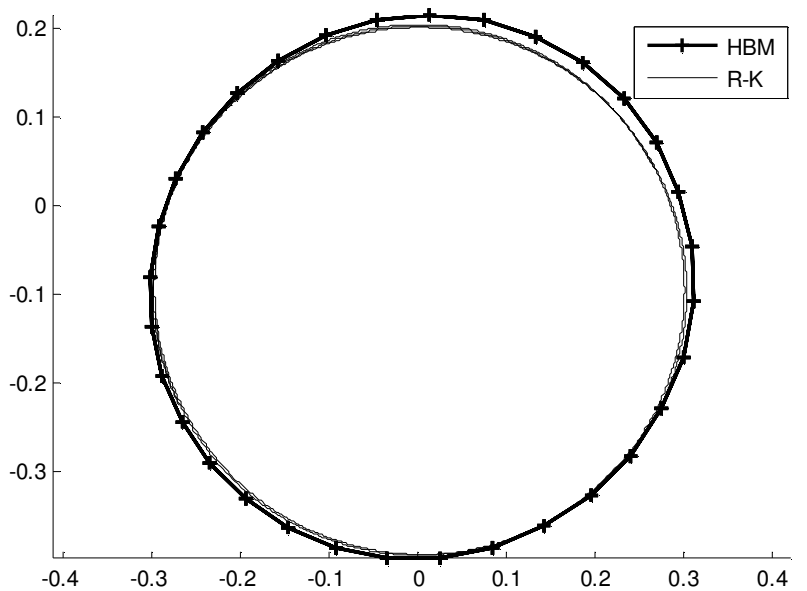

(b) $5000 \mathrm{rpm}$

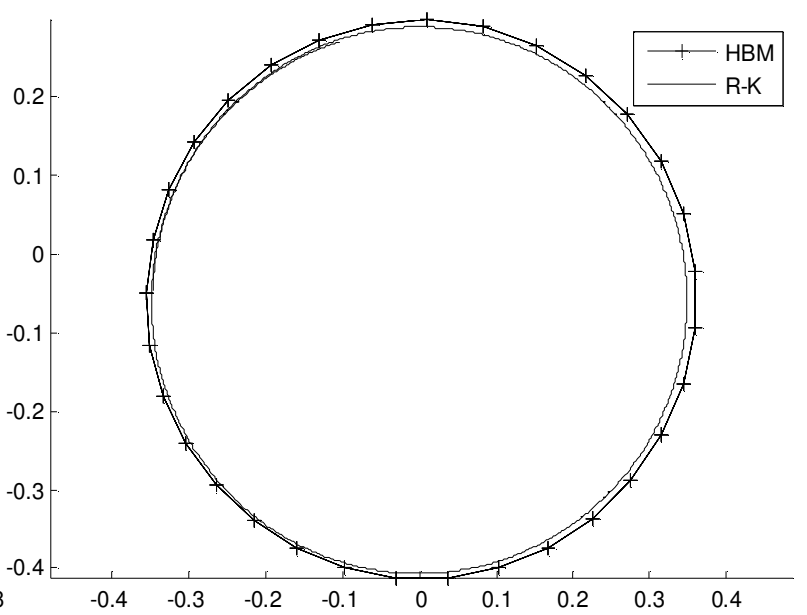

(d) $9000 \mathrm{rpm}$

Figure 6.3: Orbits of Fan-End Damper Comparison 
Table 6.1: Comparison of Computational Time

\begin{tabular}{c|c|c}
\hline \multirow{2}{*}{ Speed $($ rpm $)$} & \multicolumn{2}{|c}{ Time(Second) } \\
\cline { 2 - 3 } & HBM & Runge-Kutta \\
\hline 3500 & 125.61 & 1164.69 \\
\hline 5000 & 78.58 & 1066.49 \\
\hline 7000 & 74.98 & 433.95 \\
\hline 9000 & 79.13 & 551.7 \\
\hline
\end{tabular}

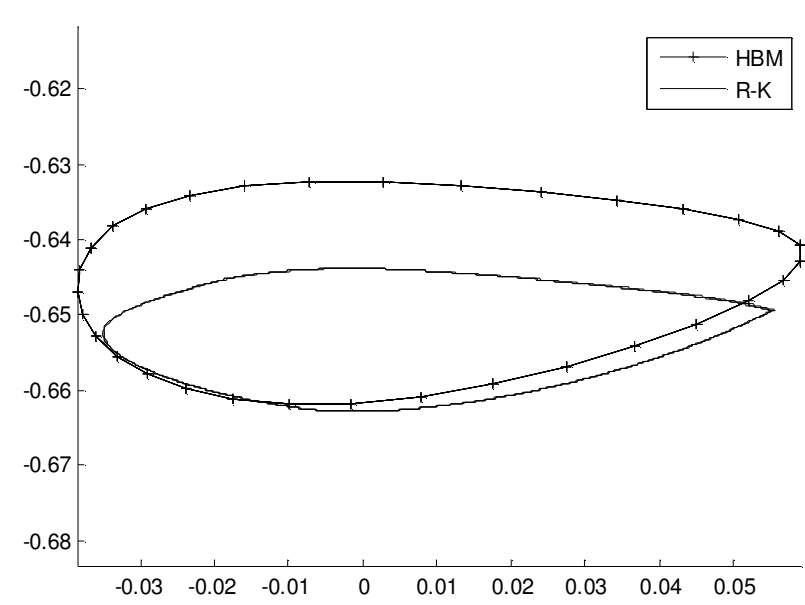

(a) $3500 \mathrm{rpm}$

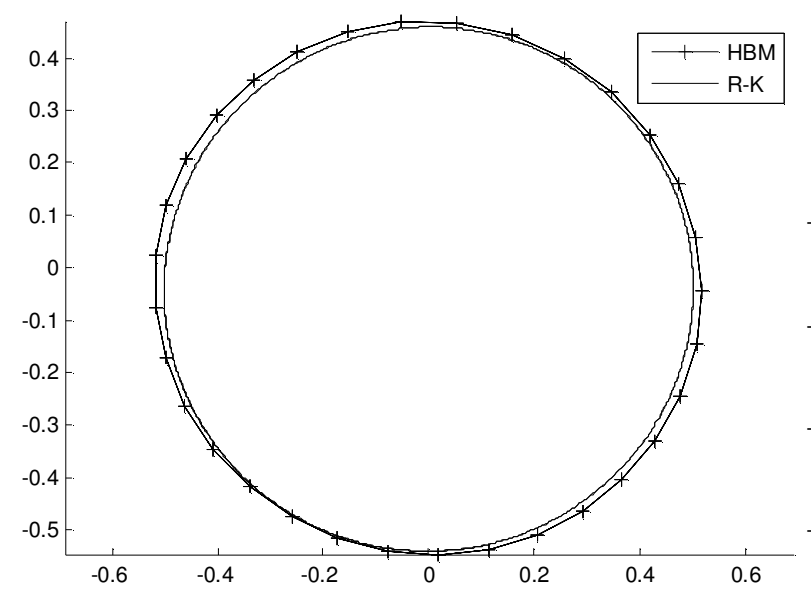

(c) $7000 \mathrm{rpm}$

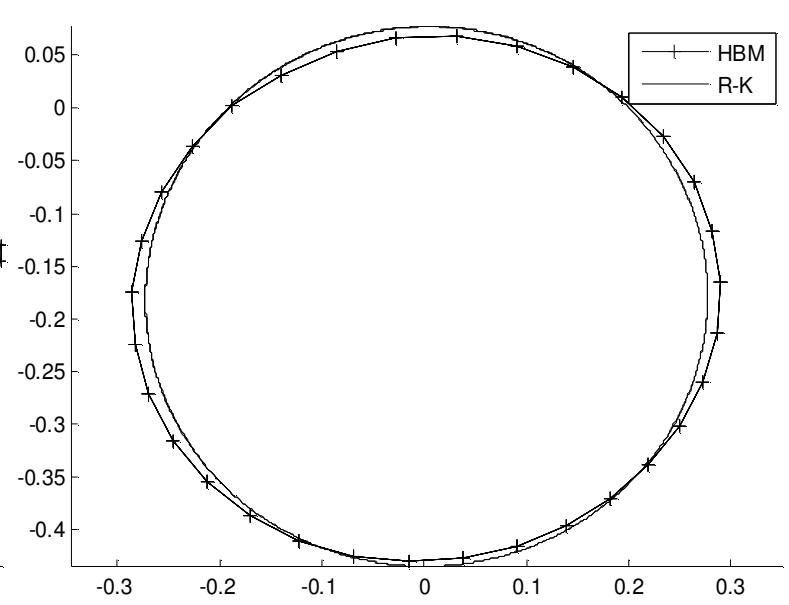

(b) $5000 \mathrm{rpm}$

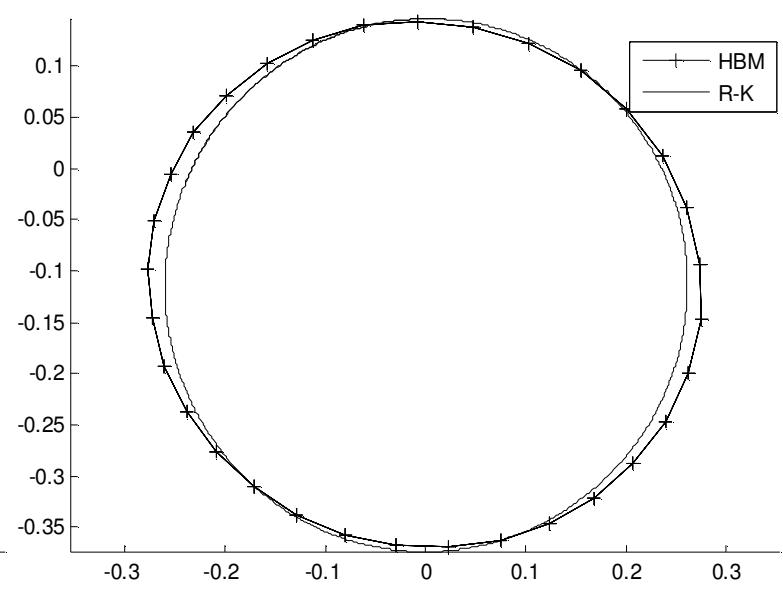

(d) $9000 \mathrm{rpm}$

Figure 6.4: Orbits of Turbine-End Damper

The HBM is 5 to 12 times more effective than the Runge-Kutta method, for these four different speeds. As discussed before, the main reason for the much longer time of RungeKutta method is that, it spends most of the time on the transient process before reaching the equilibrium. This disadvantage makes it not the first choice for steady-state analysis 
of complex rotor-bearing systems under parametric changes. As shown in the table, both methods need much more computation time for the low speed than the high one. This happens due to the presence of more nonlinearities at low speeds, which have more eccentric operating region.

With the effect of gravity, both dampers are operating eccentrically for operating speeds at 3500, 5000 and $9000 \mathrm{rpm}$. At the $7000 \mathrm{rpm}$, the orbits of the two dampers are nearly concentric, which is mainly due to the resonance around this speed. Meanwhile, the increasing running speed results in more circular orbit due to the raising unbalance force. As depicted in Figure 6.4, the elliptical response of turbine-end damper at $3500 \mathrm{rpm}$ indicates the present of super harmonics. FFT analysis given in Figure 6.5 shows that the response includes negligible $2 \mathrm{X}$ and $3 \mathrm{X}$ harmonic components. Note that, the amplitude is normalized to damper clearance, and the component from gravity is not included.

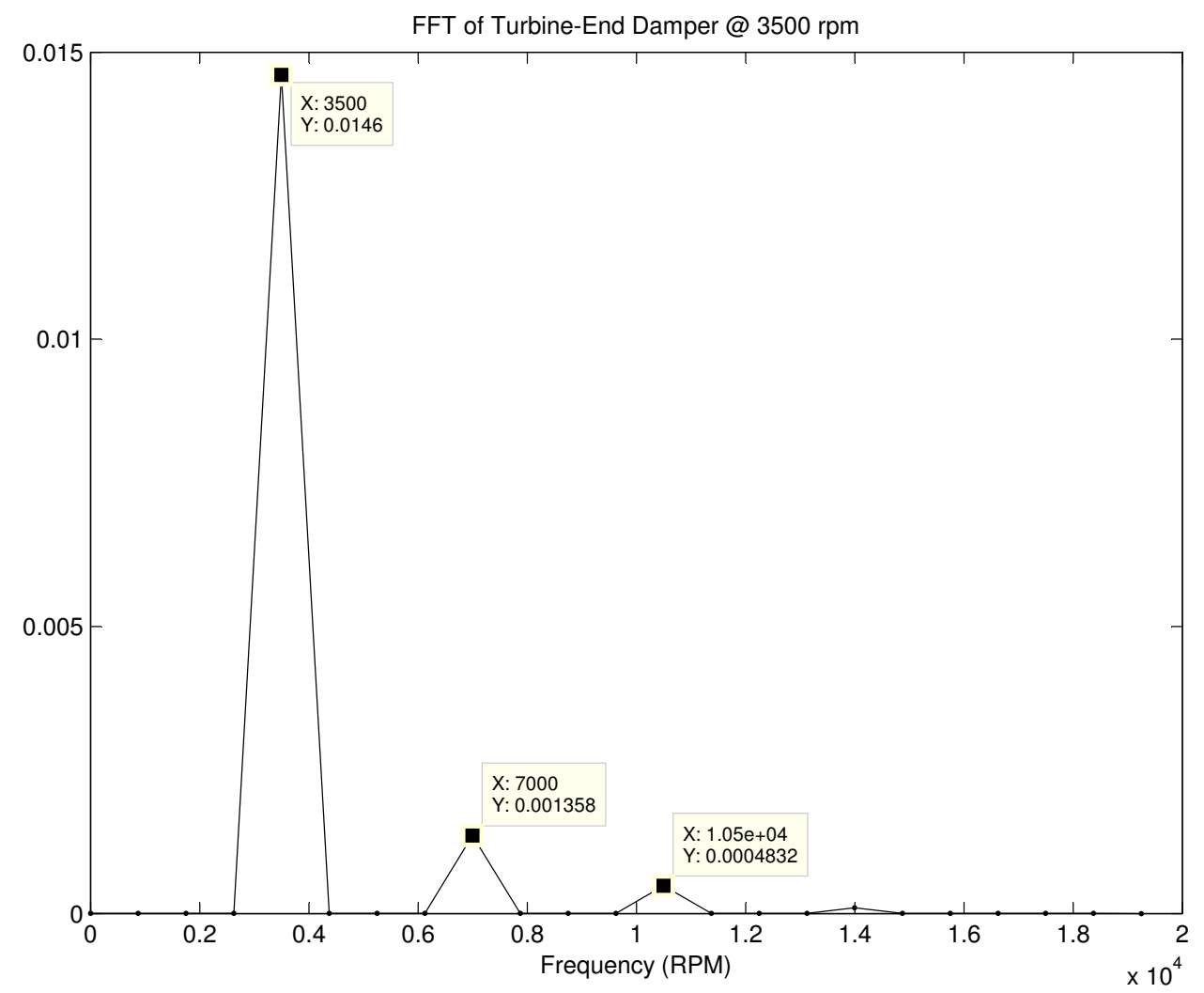

Figure 6.5: FFT of the response at $3500 \mathrm{rpm}$ 


\subsubsection{Responses with Varying Unbalance}

Different unbalance magnitudes at two different locations, fan disk and turbine disk, are applied to evaluate the effects of squeeze film damper on the responses. The magnitude changes from 2 oz-in to 6 oz-in, with phase angle fixed to $0^{\circ}$. The unbalance varies on one disk every time with the other one fixed to 2 oz-in.

\section{Varying Unbalance on Fan Disk}

For this evaluation, the fan-disk unbalance (UF) varies, while the unbalance of turbine-disk (UT) is constant. Figure 6.6 and 6.7 display the responses of fan-end damper and turbine-end damper, respectively. With small unbalance force, both dampers give responses having one critical speed around $7000 \mathrm{rpm}$. As the unbalance becomes larger, this critical speed is pushed forward to $8000 \mathrm{rpm}$, and the corresponding amplitudes is rising as well. The shifting of critical speed is caused by the increases of effective stiffness of the damper as the unbalance increases, which produces higher eccentricity. The two figure also show that the amplitude of the responses does not rise proportionally with unbalance force. The increment of amplitude for unbalance magnitude from 4 oz-in to 6 oz-in is smaller than the same unbalance change from 2 oz-in to 4 oz-in.

For response of the fan-end damper shown in Figure 6.6, another effect from the rising unbalance is the appearance of another critical speed below $7000 \mathrm{rpm}$, which is near 5000 rpm. For the turbine-end damper, as depicted in Figure 6.7, the increasing unbalance at fan-disk does not have notable effects on the responses with speed range under $4500 \mathrm{rpm}$, but the range above. Within the low speed range, the gravity load acting on turbine-end damper is larger than the unbalance forces from the rotor. Hence, the gravity dominates the response of the turbine-end damper such that the variation of unbalance forces do not have noticeable influence.

\section{Varying Unbalance on Turbine-Disk}




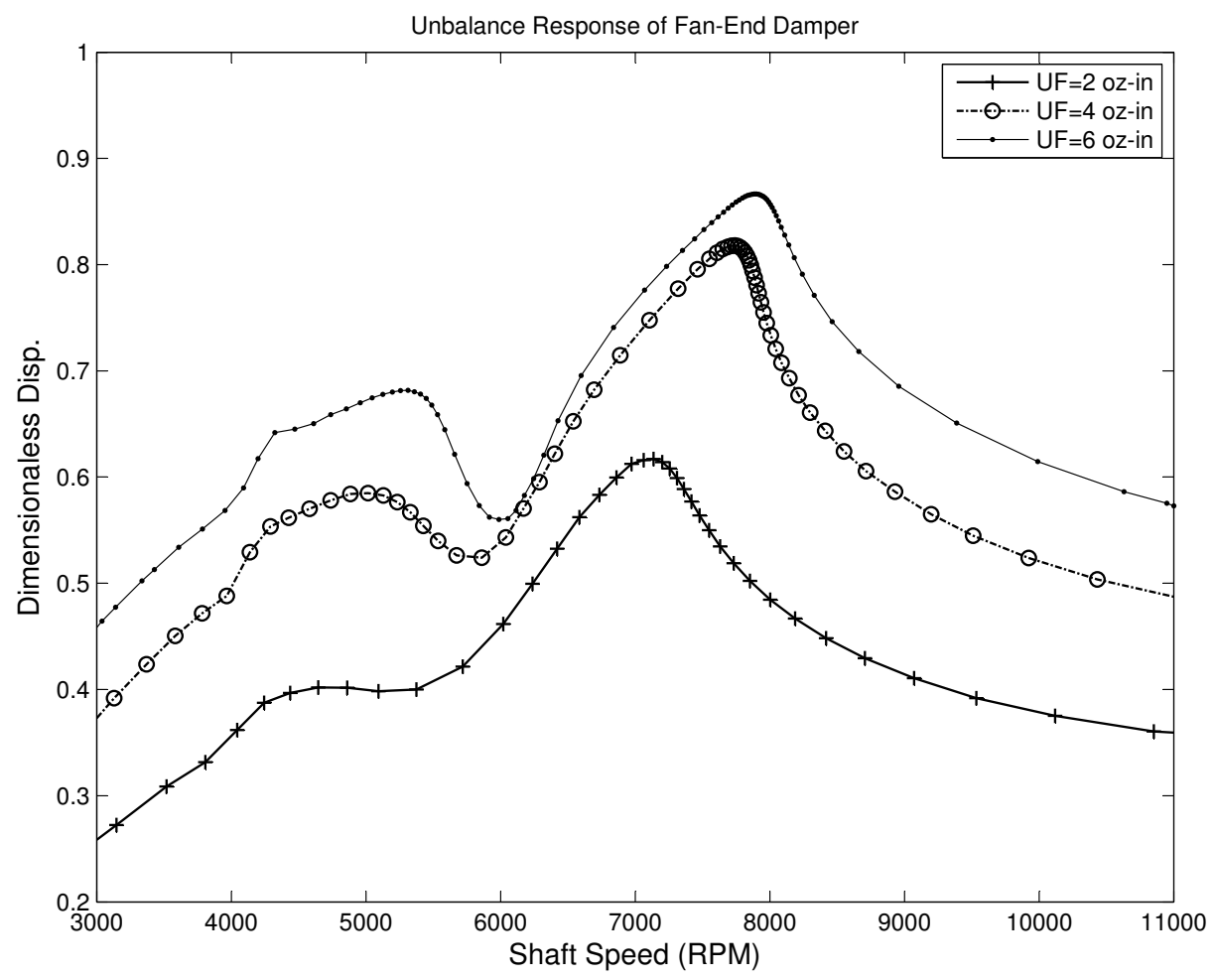

Figure 6.6: Responses of Fan-End Damper, UT=2 oz-in

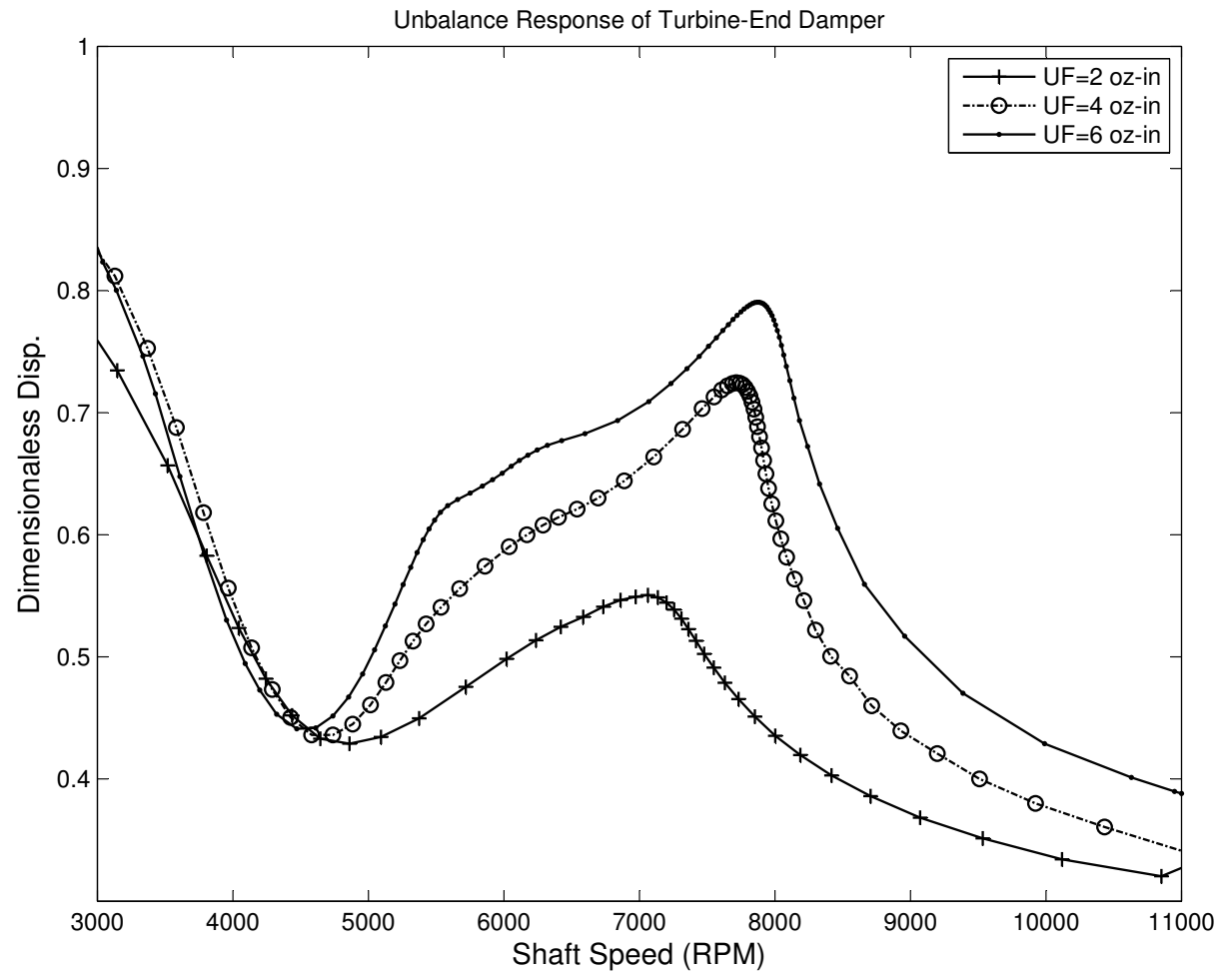

Figure 6.7: Responses of Turbine-End Damper, UT=2 oz-in 
Figure 6.8 and 6.9 display forced responses of fan-end damper and turbine-end damper with changing unbalance on turbine-disk. The unbalance loads at turbine disk vary from 2 to 8 oz-in, with constant in-phase unbalance of 2 oz-in at fan-disk. As shown in Figure 6.8, the reaction of fan-end damper is similar to the cases in Figure 6.6 for speed range over $6000 \mathrm{rpm}$. But it is less sensitive in the sense that another critical speed starts to appear not until 8 oz-in, comparing to 4 oz-in for the previous case. For the speed range below 5000 rpm, the responses are decreasing with increasing unbalance. The main reason is that the unbalance force counteracts gravity and lifts the rotor to an area where gravity has less effect. This phenomenon can also be observed at turbine-end damper within low speed range when unbalance UT is smaller than 6 oz-in, as depicted in Figure 6.9. Once the unbalance rises to 8 oz-in, the unbalance force becomes dominated over the low speed range, creates another critical speed around $5000 \mathrm{rpm}$, and generates concave range with smaller amplitude around 6500 rpm than other cases.

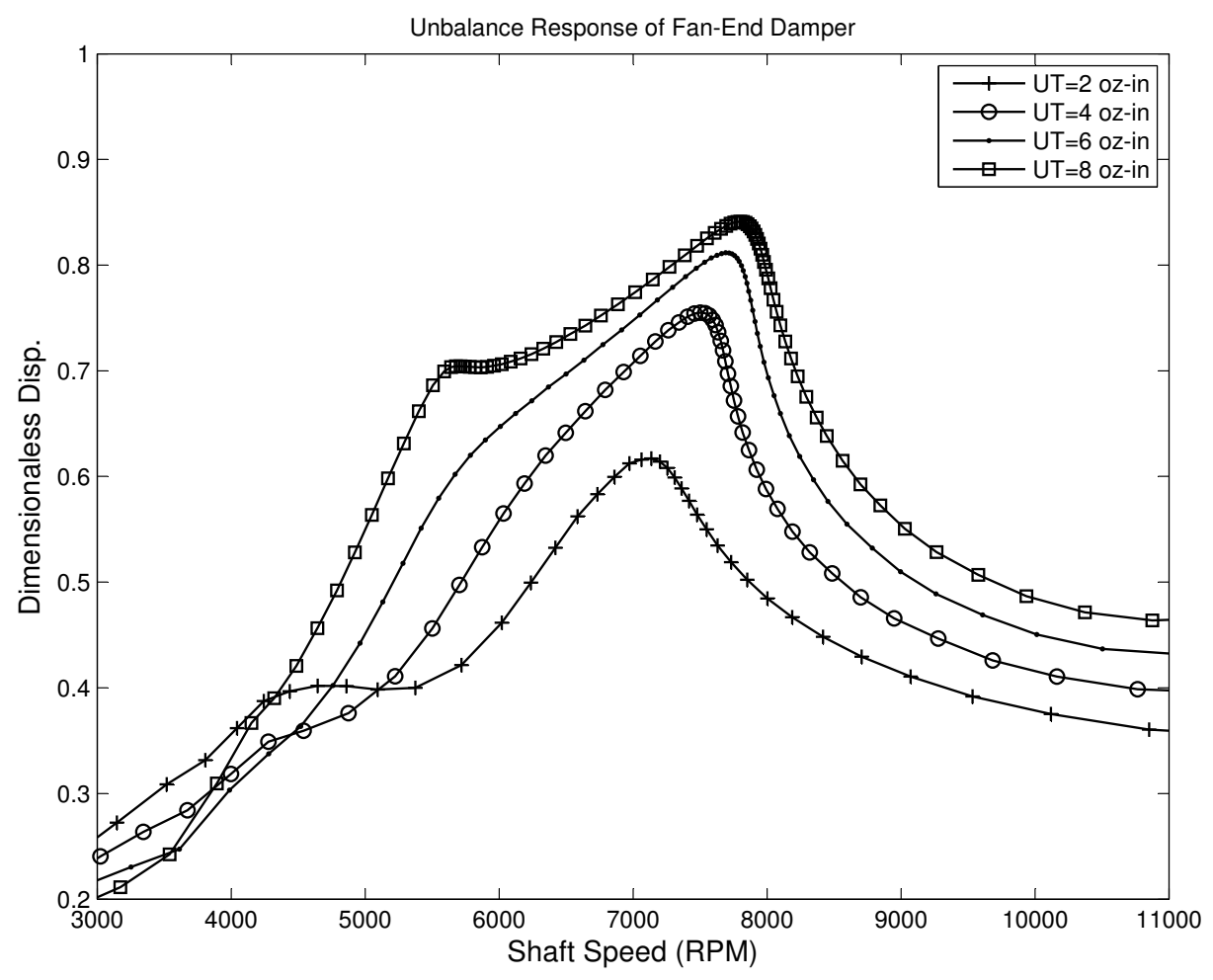

Figure 6.8: Responses of Fan-End Damper, UF=2 oz-in 


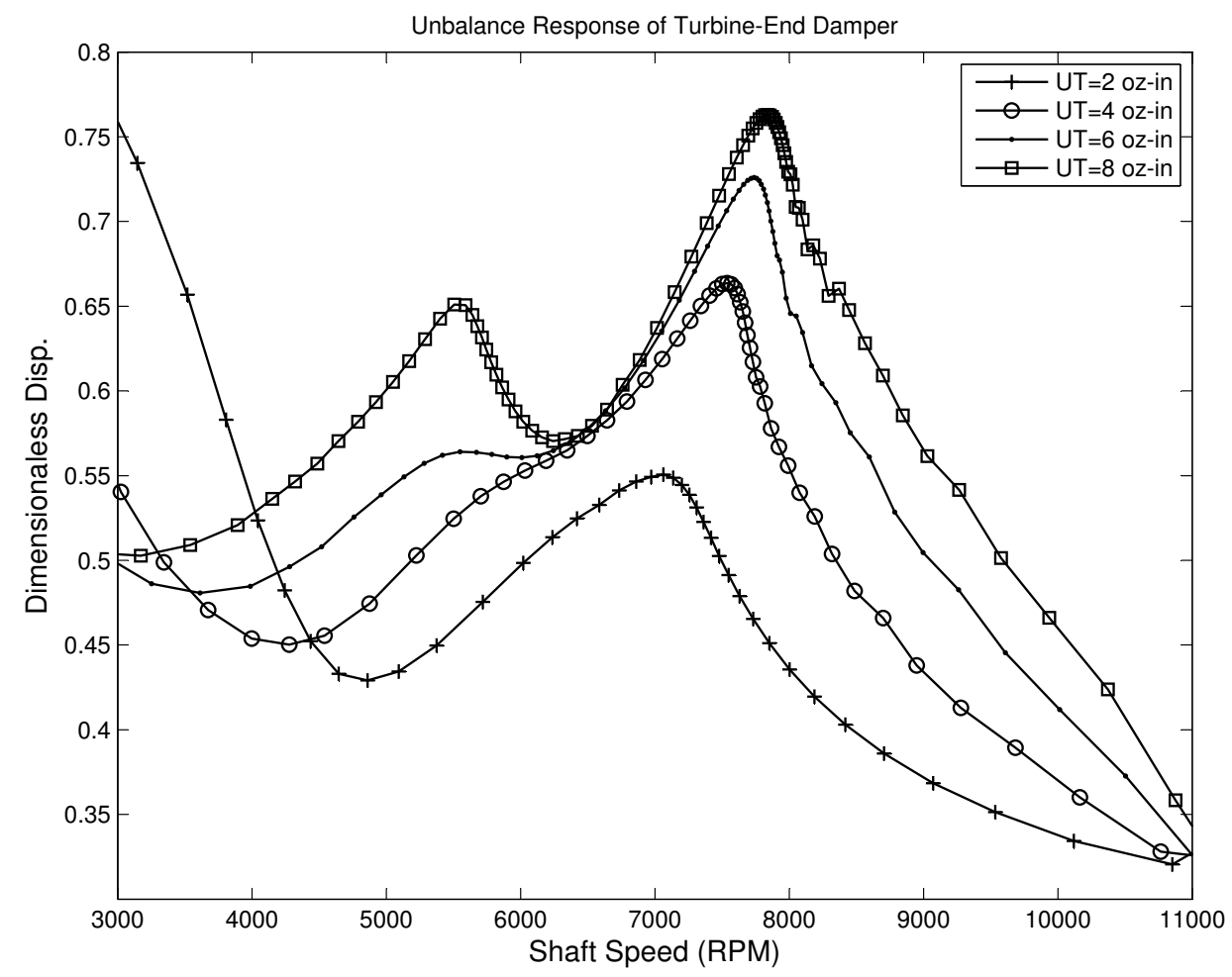

Figure 6.9: Responses of Turbine-End Damper, UF=2 oz-in

\subsubsection{Responses with Varying Spring Stiffness}

The effects of centering spring stiffness of damper on the unbalance are evaluated in this part. The value of spring stiffness is scaled to that of the ball bearing next to the fan-end damper.

\section{Varying spring stiffness of fan-end damper}

The responses corresponding to the variation of spring stiffness at fan-end damper (KF) are depicted in Figure 6.10 and 6.11 for fan-end damper and turbine-end damper, respectively. For the responses of the journal at fan-end, the stiffer centering spring increases the critical speed, raises the amplification factor, and decreases the responses of speeds out of the critical range. For the turbine-end damper, the changing stiffness at fan-end damper does not have much effect for speed range lower than $4000 \mathrm{rpm}$. While the response amplitude increases with stiffness over the range between $4000 \mathrm{rpm}$ and $5000 \mathrm{rpm}$ before decreasing. However, the turbine-end damper is less sensitive to the stiffness variation over the operating range larger than 9000 rpm. 


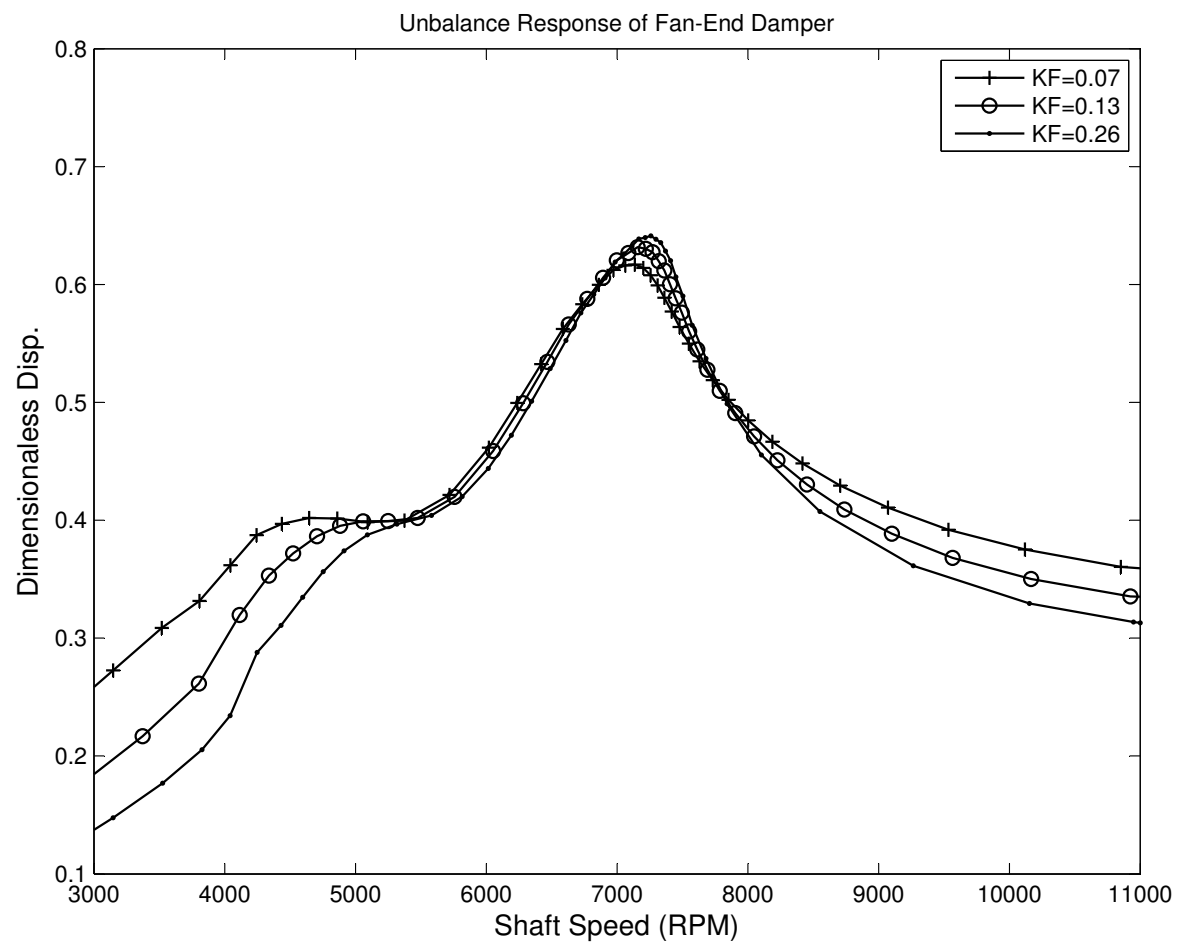

Figure 6.10: Response of Fan-End Damper with varying KF

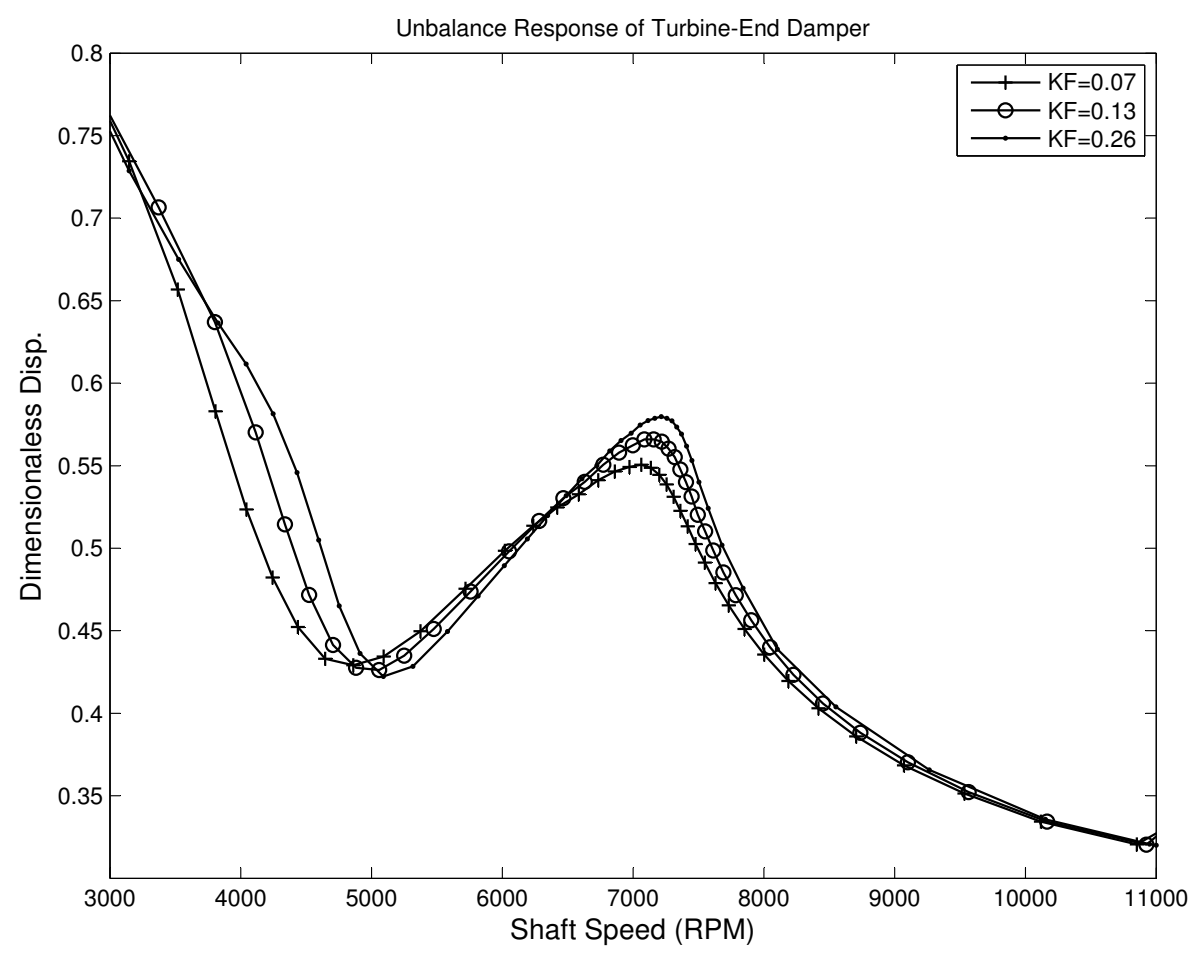

Figure 6.11: Response of Turbine-End Damper with varying KF 


\section{Varying spring stiffness of turbine-end damper}

Figure 6.12 and 6.13 display the responses of both dampers under the varying spring stiffness of turbine-end damper (KT). As shown by Figure 6.12, the responses of most speeds within the studied range does not vary significantly with the spring stiffness at turbine-end, except the flat transition between $4000 \mathrm{rpm}$ and $5000 \mathrm{rpm}$. One major difference from responses under varying stiffness of fan-end damper $(\mathrm{KF})$ is that, the stiffer spring of turbineend damper does not increase the critical speed of the system, but remains unchanged as depicted in the two figures.

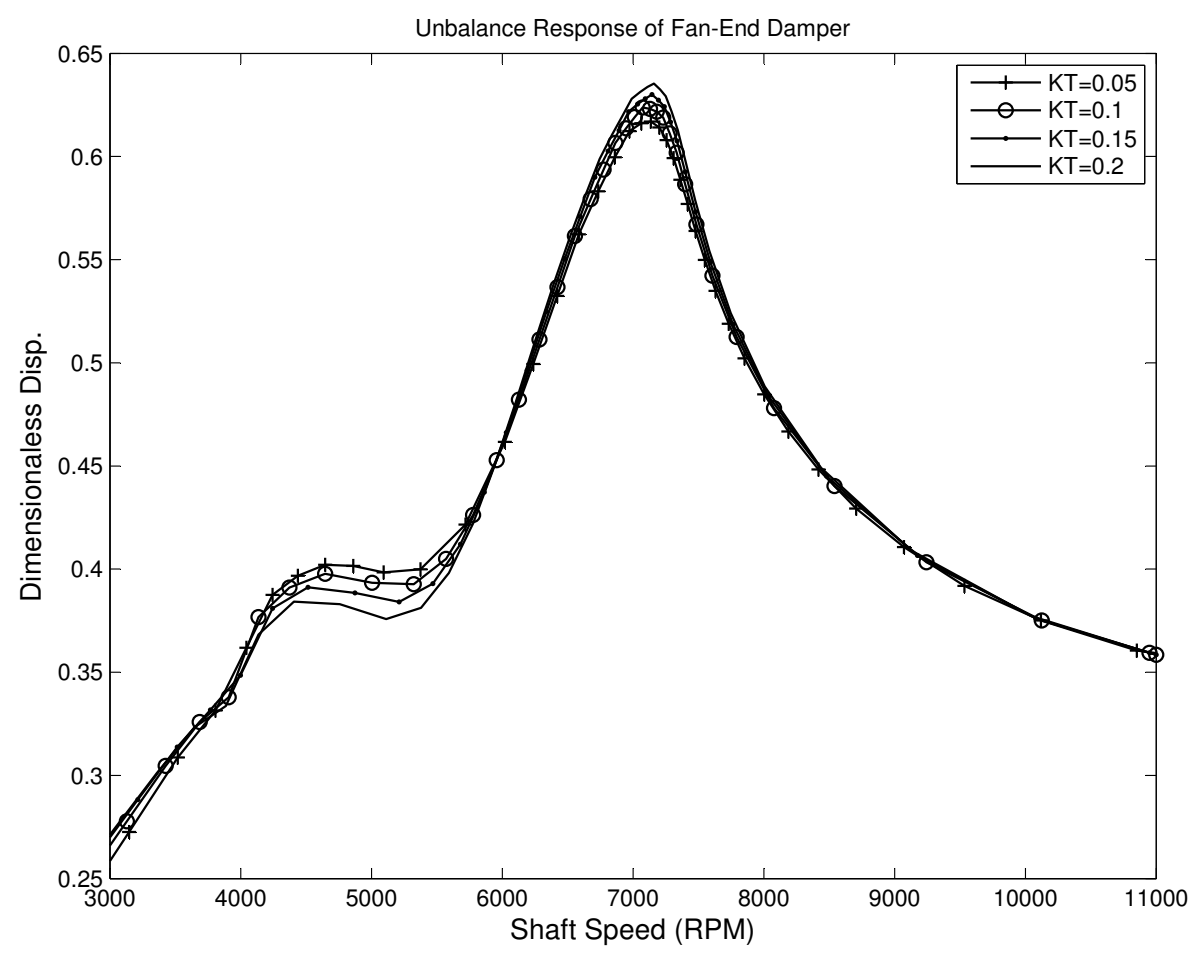

Figure 6.12: Response of Fan-End Damper with varying KT

For the turbine-end damper, the harder spring not only decreases the response amplitude for speed range away from critical speed, but also reduces magnitude around 7000 rpm, contrast to all other responses in figures from 6.10 to 6.12. As the centering spring of the turbine-end damper becomes stiffer, it supports more rotor weight and lifts the equilibrium position from the larger eccentricity region, where the damper produces higher equivalent 


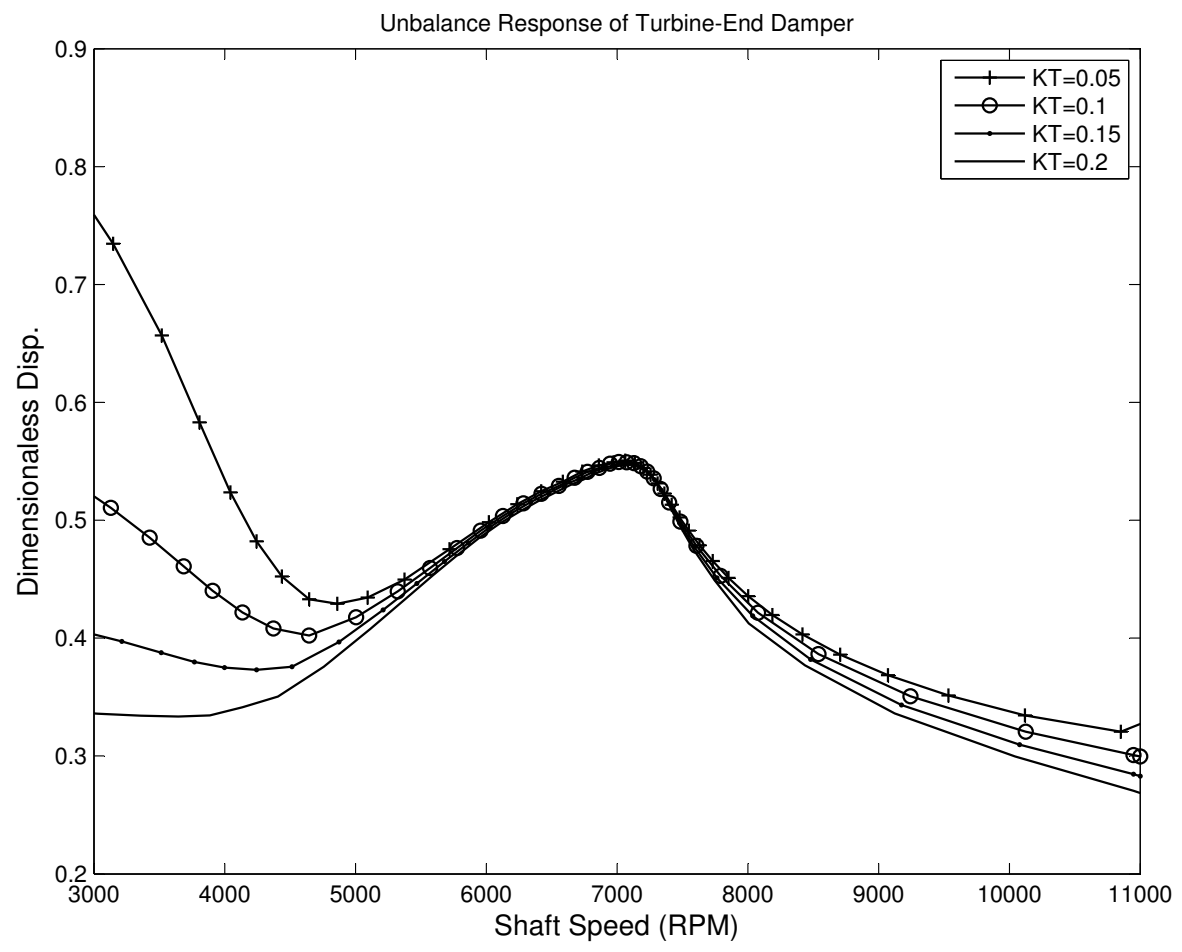

Figure 6.13: Response of Turbine-End Damper with varying KT

stiffness that diminishes the motion of damper. Hence, with less equivalent stiffness, the corresponding damping acts more effectively such that the amplitude of unbalance response with stiff spring is lower than that with soft centering spring.

\subsection{Rotordynamics of the High Pressure Rotor}

The model of the High Pressure rotor uses the same method for the analysis of LP rotor. Figure 6.14 gives the schematic view and FEA model of the rotor [121]. The rotor is discretized into 24 nodes. A roller bearing in-series with damper supports the left end of the rotor, and the other ball bearing is located at node 20. It has total weight around $210 \mathrm{lbf}$ and its running speed is about 15,000 rpm.

The rotordynamics analysis of this rotor focuses on the effects of SFDs with piston ring seal, since it is one of the most popular sealing arrangements for SFDs in aero-engines. A simplified model of the piston ring from Gunter et al. [1] will be used to study the unbalance 


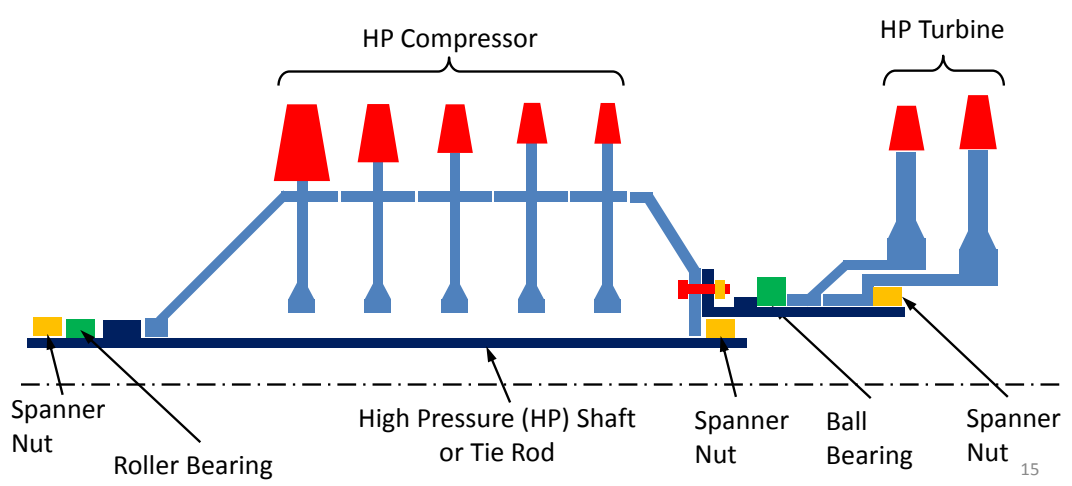

(a) Schematic View of HP Shaft

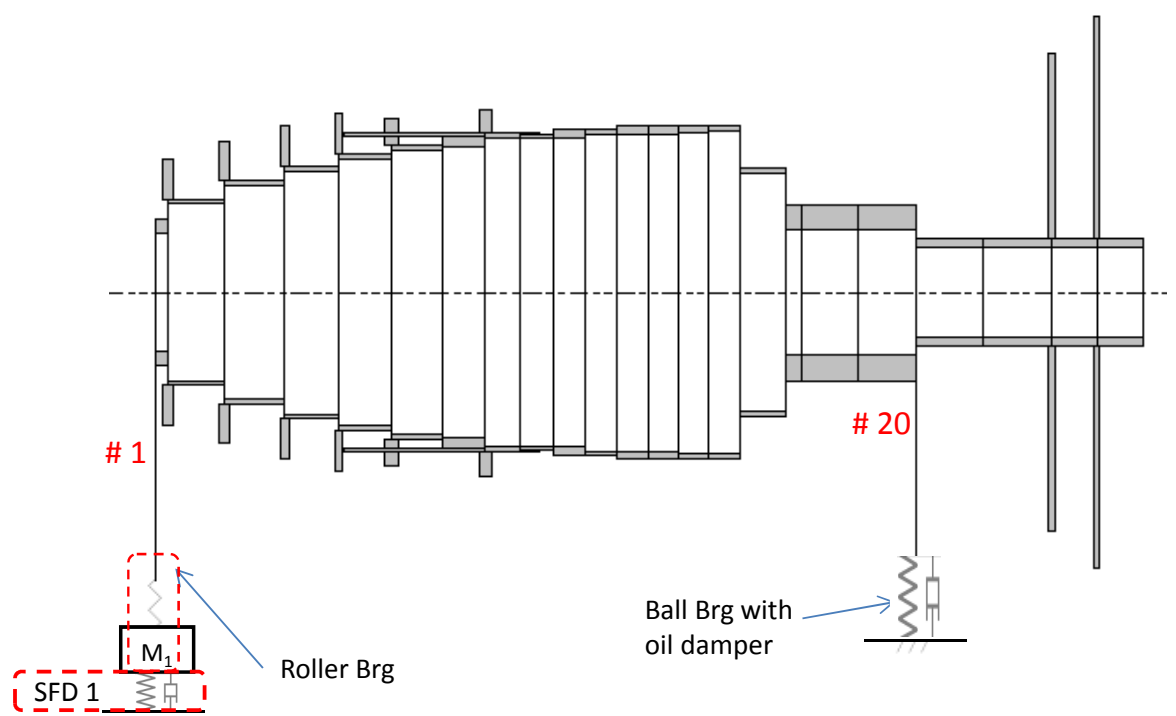

(b) Finite Element Model of HP Shaft

Figure 6.14: Schematic View and FEA Model of HP Rotor

responses and stability of the rotor. Two typical assemblies of this kind of seals, piston ring with central groove supply and direct hole supply, will be modeled using the finite difference method developed in chapter 3 .

\subsubsection{Rotordynamics with Piston Ring Seals - a Simplified Model}

The simplified model for the piston ring is the one discussed in part 4.1.2 of chapter 4 . It assumes no leakage happens at the end and the pressure inside the central groove is zero[1]. With these assumptions, the SFDs are considered as equivalent to open-end dampers, therefore, the force coefficients method is able to model these kinds of SFDs. The effects of 
varying damper parameters, including aspect ratio $L / D$, clearance ratio $C / R$ and centering spring stiffness, on the rotordynamics of the HP rotor are studied. The stability margin of the rotor subject to cross-coupled stiffness from the axial compressor is also documented.

\subsubsection{Responses with Varying Parameters of SFDs}

The rotor is excited by one unbalance force acting on the second stage of the turbine, with magnitude of $4 W / N$ and phase of $0^{\circ}$. The diameter of the journal is 5.512 inch for all the varying cases. No cross-coupled stiffness is applied for the unbalance responses. The force coefficients method with the Curve Intersection procedure from chapter 4 is used for the analysis.

\section{1) Effects of Varying Aspect Ratio}

The range of $L / D$ is studied from 0.075 to 0.25 , which is split into eight cases. The clearance ratio $C / R$ and centering spring stiffness are $1 / 1000$ and $1.75 \mathrm{e} 5 \mathrm{lb} / \mathrm{in}$, respectively, for all the cases. The film is assumed to be un-cavitated, in other words, $2 \pi$-film is used.

Figure 6.15 shows the damper responses with varying aspect ratio. For the entire speed range, the smaller $L / D$ the higher the damper responses. All eight cases have similar critical speed value around 8,000 and 14,000 rpm. The first critical speed is more sensitive to the variation of aspect ratio below 0.15 than the one above this value. While the second critical speed behaves the opposite way.

From the figure, these two critical speeds do not vary much with different L/D, which indicates the effective stiffness of the damper does not change much with varying $\mathrm{L} / \mathrm{D}$. The main contribution for the increase of $\mathrm{L} / \mathrm{D}$ is the damping, as evidenced by the amplitude reduction around the two critical speeds. Especially for the first critical speed, the amplification factor decreases dramatically with increasing L/D. When L/D is larger than 0.2 , the amplification factor becomes negligible. In other words, the first critical speed is eliminated. The responses near the second critical speed also decrease the amplification factor, but the decreasing rate is much smaller than that for the first critical speed. For L/D larger than 0.2, 
the damper generates more damping than necessary and starts to lock the journal motion, which results in the right shift of the second critical speed.

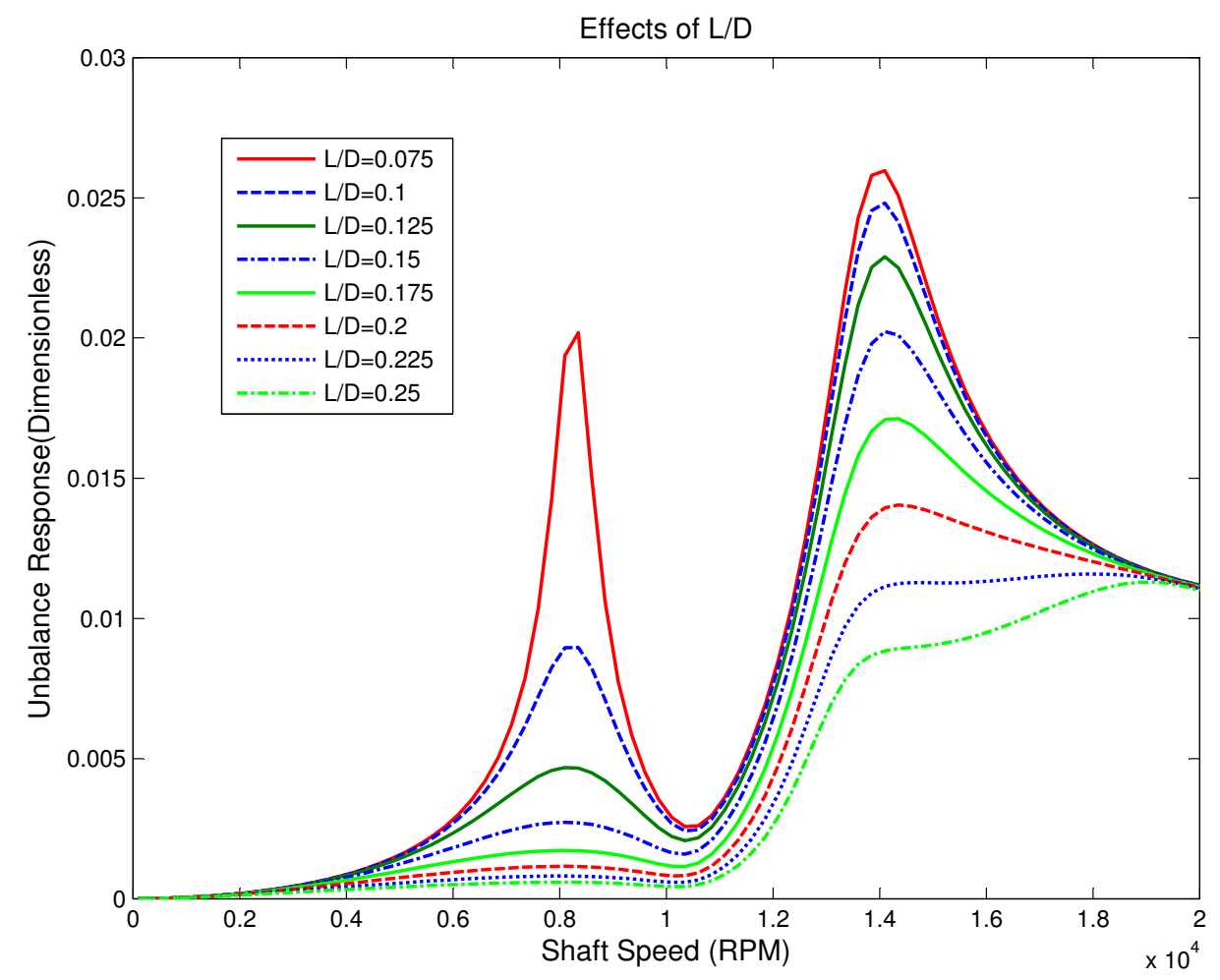

Figure 6.15: Damper Responses with Varying L/D

\section{2) Effects of Varying Clearance Ratio}

The variation range of $C / R$ is from $0.75 / 1000$ to $2.5 / 1000$, with $L / D=0.2$ and centering spring stiffness of $1.75 \mathrm{e} 5 \mathrm{lbf} / \mathrm{in}$. Similar to the study of aspect ratio, the film is assumed to be $2 \pi$-film.

The responses with varying clearance ratio are shown in Figure 6.16. There are also two critical speeds within the studied speed range, around 8,000 rpm and 14,000 rpm, which are the same as Figure 6.15. Different from the effects of varying aspect ratio, the amplitude of the first critical speed increases with the clearance ratio. The higher value of $C / R$ produces less damping than the lower value, therefore, increases the amplification factor. When $C / R$ equals to the smallest value of $0.75 / 1000$, the first critical speed is almost damped out. 
The amplitude of the second critical speed behaves opposite from the first critical for the range of clearance ratio between $1.25 / 1000$ to $2.5 / 1000$. The higher value of the ratio, the less amplitude and smaller amplification factor of the response. The range of clearance ratio below 1.25/1000 gives smaller responses than the range above. The ratio of 1/1000 generates the largest amplification factor over the entire range. As the clearance ratio reaches the smallest value of $0.75 / 1000$, the second critical speed shifts to the right.

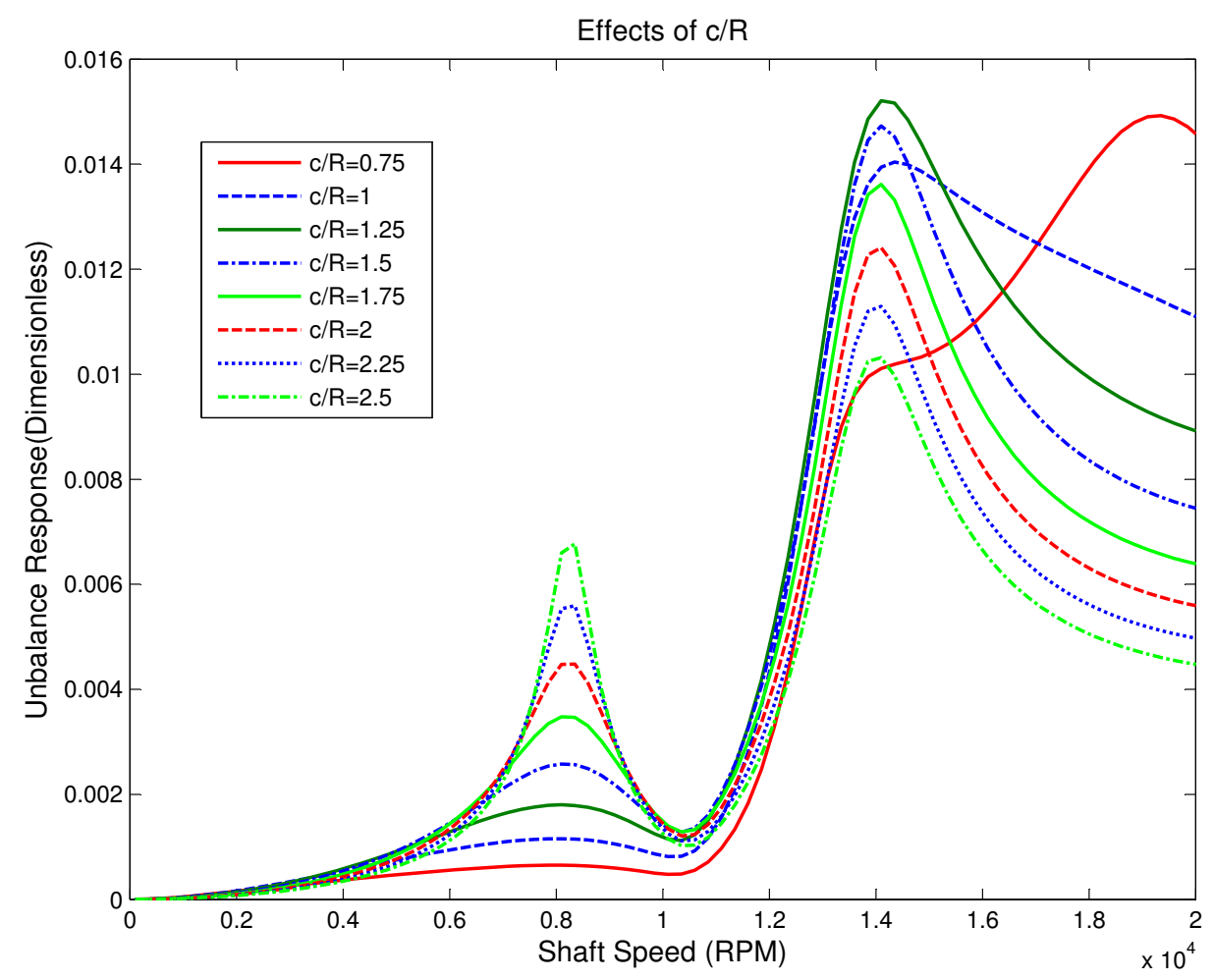

Figure 6.16: Damper Responses with Varying $\mathrm{C} / \mathrm{R}\left(10^{-3}\right)$

\section{3) Effects of Varying Centering Spring Stiffness}

The centering spring stiffness changes from $7.5 \mathrm{e} 4$ to $2.5 \mathrm{e} 5 \mathrm{lbf} / \mathrm{in}$, with aspect ratio and clearance ratio fixed to 0.2 and $1 / 1000$, respectively. The effect of the varying stiffness on the responses is depicted in Figure 6.17. The figure shows the variation range of the stiffness does not have significant influence on the responses, although the hardest stiffness is three times more than the softest one. 
The responses with soft spring stiffness are slightly higher than those with stiffer spring, when the operating speed is below the first critical speed. All the responses are nearly the same as crossing the first critical speed. For speeds near the second critical, the differences among each case are more distinguishable. The higher centering spring stiffness gives higher responses, opposite to the first critical speed. Moreover, the amplification factor is found to increase with stiffness for the studied range, which indicates the effective damping with stiffer spring is larger than that with softer spring for the speeds around and above the second critical speed.

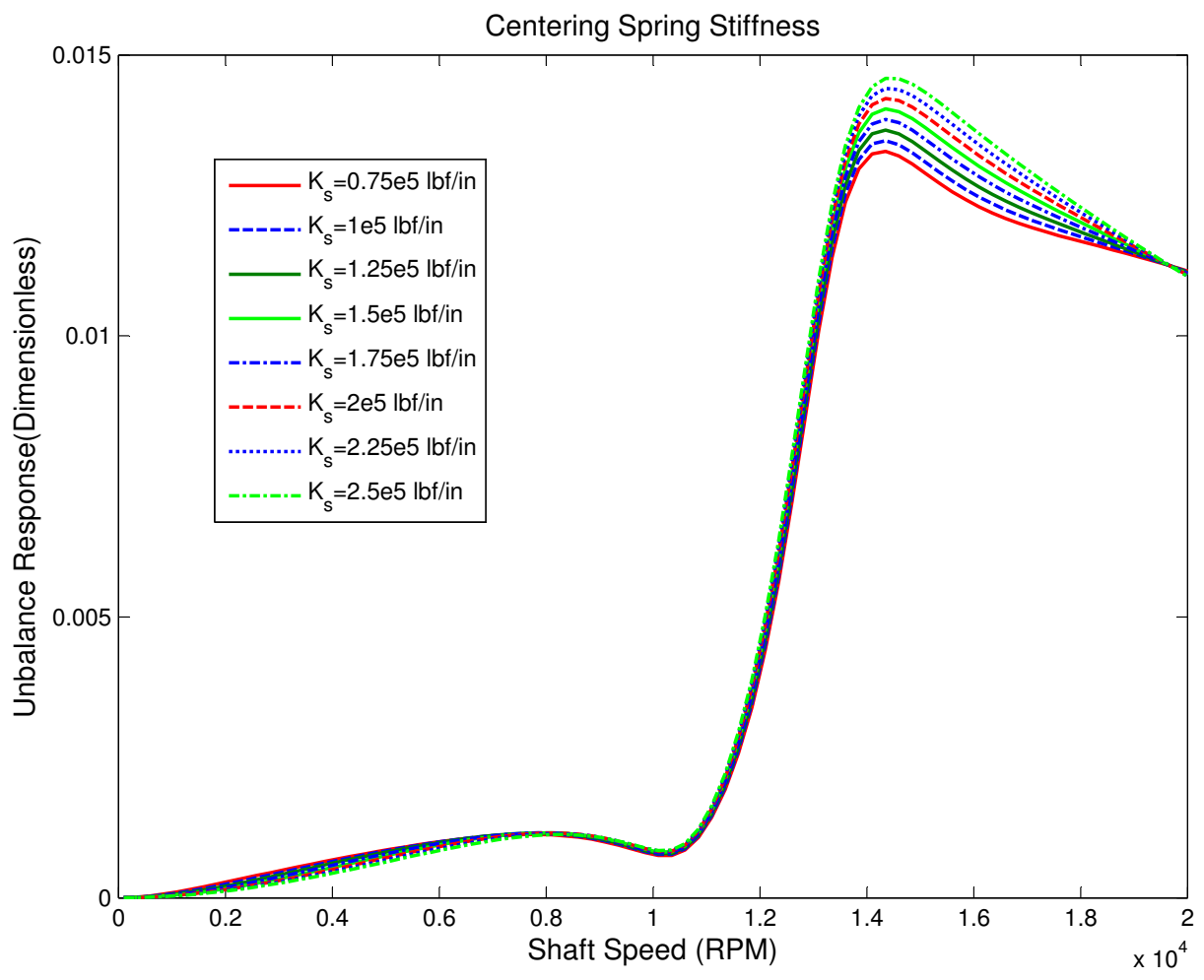

Figure 6.17: Damper Responses with Varying Spring Stiffness

\subsubsection{Stability Margin Subject to Cross-coupled Stiffness}

As discussed in chapter 4, the cross-coupled stiffness has great effect on the stability of rotating systems. Within the high pressure rotor of aero-engines, the main source of the cross-coupled stiffness comes from the aerodynamic cross-coupling in the high pressure 
compressors. These kind aerodynamic forces can cause self-excited vibration, which leads to instability[122, 123, 124, 125]. This part will study effects of SFDs on the stability of the HP rotor subject to aerodynamic forces. Similar to the unbalance responses analysis, three parameters, aspect ratio, clearance ratio and centering spring stiffness will be discussed. For all the cases, the cross-coupled forces from all the stages of the compressor are approximated as the forces acting on stage seven only.

\section{1) Stability Margin with Varying Aspect Ratio}

The variation range of aspect ratio is the same as before, from 0.075 to 0.25 , with clearance ratio and centering spring stiffness fixed to $2 / 1000$ and $1.75 \mathrm{e} 5 \mathrm{lbf} / \mathrm{in}$, respectively. Figure 6.18 and 6.19 show the natural frequency and logarithmic decrement of the HP rotor with different aspect ratio of the SFD. The natural frequency of the system is found to decrease with $\mathrm{L} / \mathrm{D}$, as presented in Figure 6.18. The frequency at $L / D=0.075$ gives about 1000 cpm higher than that of $L / D=0.25$, which indicates the effective stiffness of the damper decreases with the aspect ratio. The decreasing rate of the frequency is the most sensitive to L/D between 0.15 and 0.225 .

Figure 6.19 presents the logarithmic decrement with aspect ratio. The logarithmic decrement increases with the ratio before starting to decrease at 0.2 . The maximum logarithmic decrement is 0.57 , compared to 0.32 of the minimum value. Different from the change of natural frequency, this variation trend indicates the effective damping of the system does not behave monotonically. It has an optimized value of the logarithmic decrement, which gives the maximum stability margin for the HP rotor. This conclusion can also be observed from Figure 6.20. The figure shows the maximum cross-coupled forces the HP rotor can resist before becoming unstable for different aspect ratio. It can be found that the figure gives the same trend of the stability behavior of the rotating system, as shown in Figure 6.19. The maximum cross-coupled stiffness is about $4.5 \mathrm{e} 5 \mathrm{lbf} / \mathrm{in}$, and the minimum is about $8.5 \mathrm{e} 4$ $l b f / i n$. 


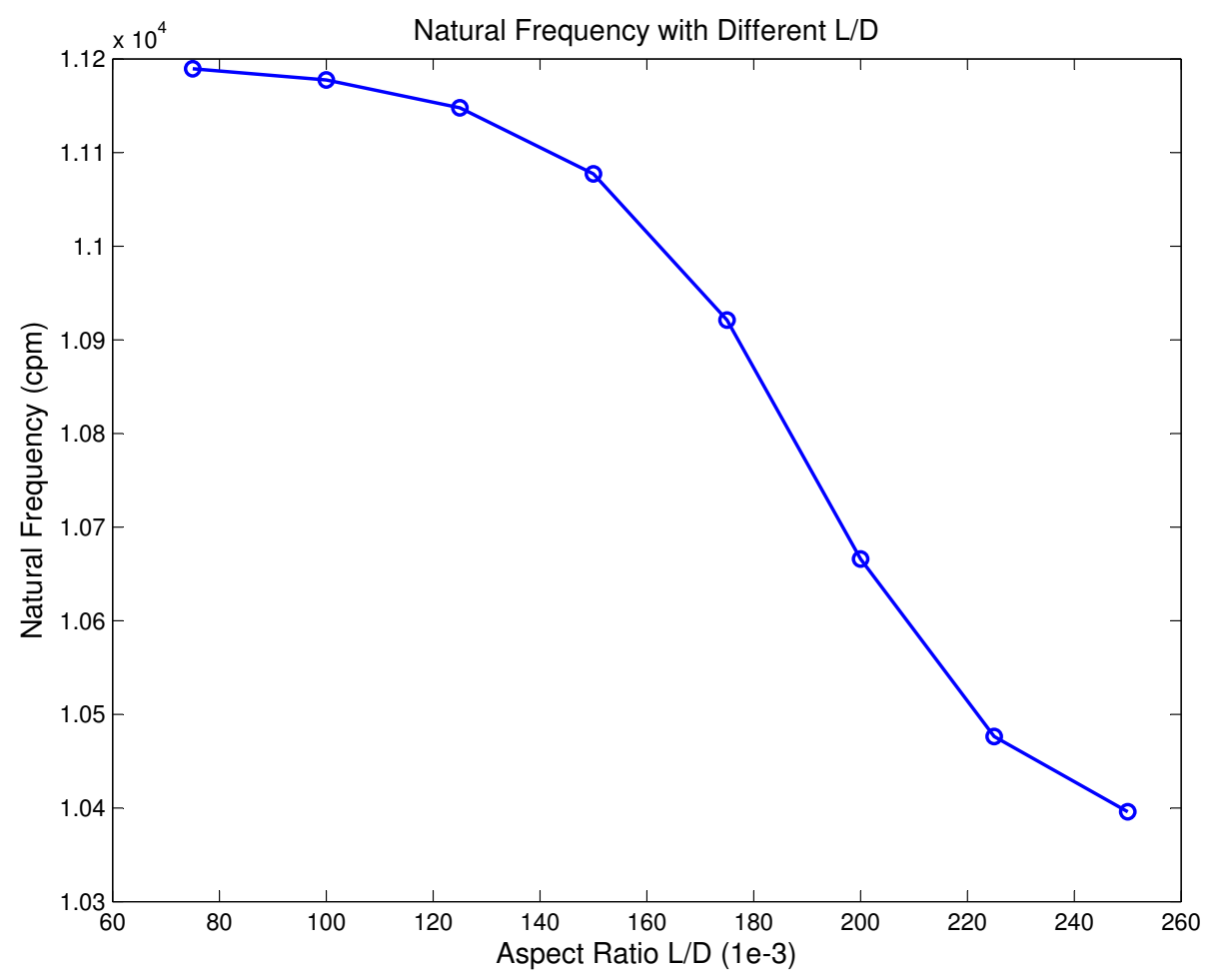

Figure 6.18: HP Rotor Natural Frequency with Varying Aspect Ratio

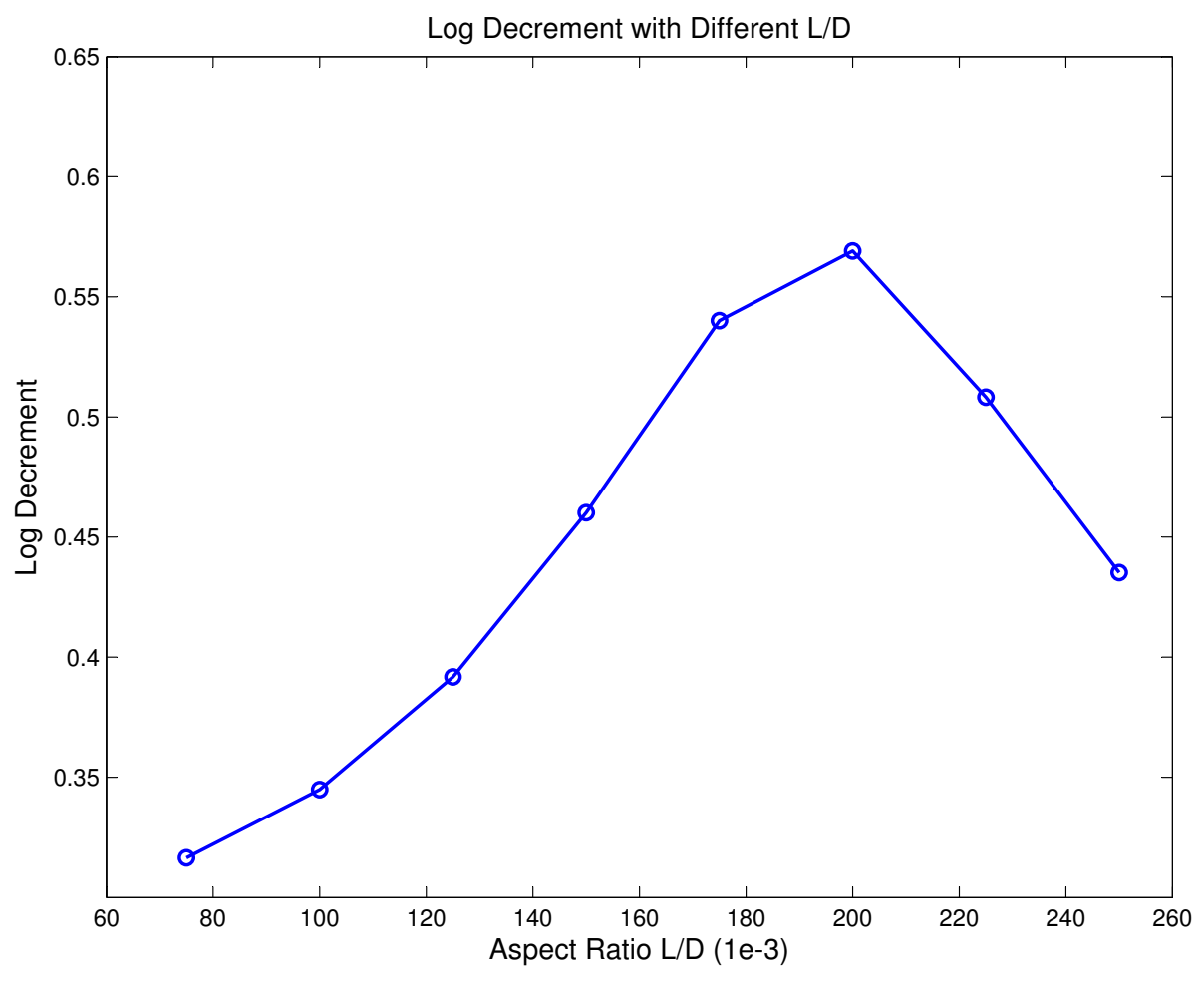

Figure 6.19: HP Rotor Log Decrement with Varying Aspect Ratio 


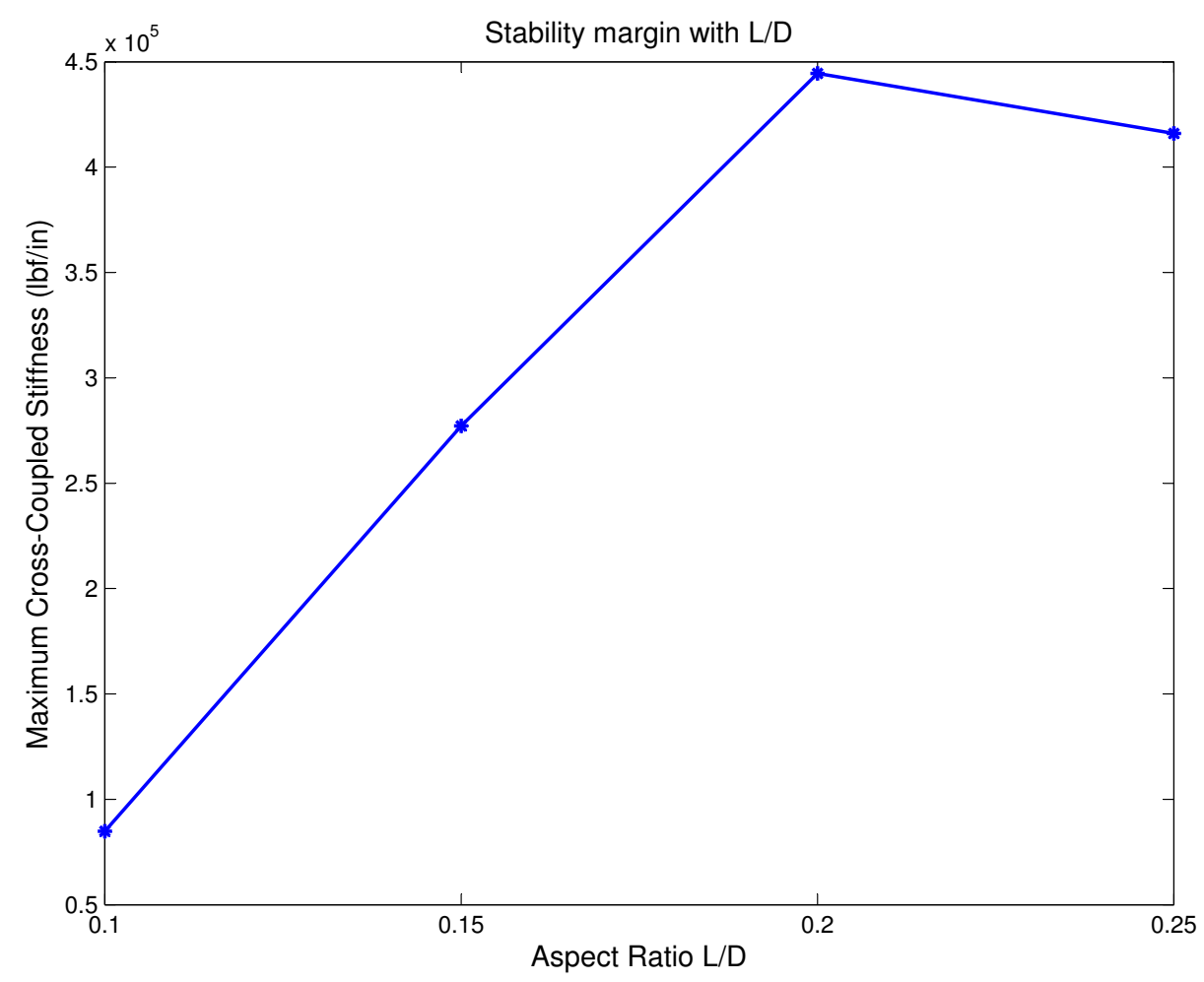

Figure 6.20: Stability Margin with Varying Aspect Ratio

\section{2) Stability Margin with Varying Clearance Ratio}

The range of clearance ratio $(\mathrm{C} / \mathrm{R})$ is from $0.75 / 1000$ to $2.5 / 1000$, with $\mathrm{L} / \mathrm{D}$ and spring stiffness fixed to 0.2 and $1.75 \times 10^{5} \mathrm{lbf} / \mathrm{in}$, respectively. Figure 6.21 gives the stability margin with the clearance variation. The margin reaches its maximum at $\mathrm{C} / \mathrm{R}=1 / 1000$ before decreasing. The maximum cross-coupled stiffness is about ten times as large as the minimum when $\mathrm{C} / \mathrm{R}=2.5 / 1000$. This difference is much larger than that with the variation of aspect ratio, which indicates the stability of HP rotor is more sensitive to the clearance ratio than the aspect ratio.

Figures 6.22 and 6.23 give the natural frequency and logarithmic decrement subject to the variation of clearance ratio. Same as the stability margin shown in Figure 6.21, the logarithmic decrement decreases with the ratio for most range of the variation, although it increases for the ratio below $1 / 1000$. It drops quickly right after the maximum value and decreases gradually with larger clearance. 


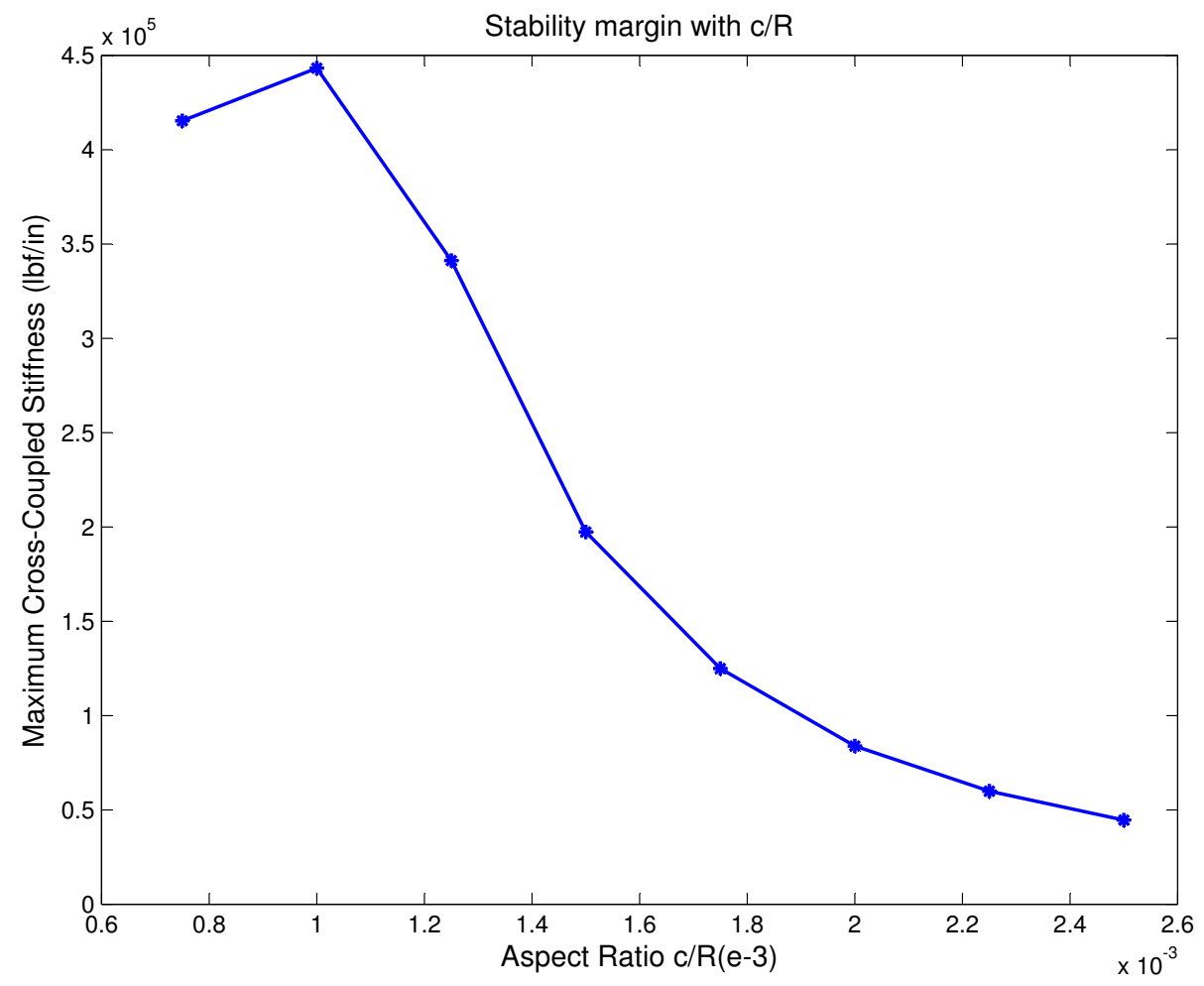

Figure 6.21: Stability Margin with Varying Clearance Ratio

Figure 6.22 represents the influence of damper clearance on the natural frequency of the HP rotor. The frequency is found to increase with $C / R$, which is different from the relation with $L / D$. It is more sensitive to the clearance range between $0.75 / 1000$ and $1.25 / 1000$ than the rest. The sensitive range gives $660 \mathrm{cpm}$ variation of the natural frequency, which is about $80 \%$ of total variation. As clearance increases above 1.5/1000, the natural frequency does not rise that much. The frequency difference between $C / R=2.25 / 1000$ and $C / R=2.5 / 1000$ is only about $10 \mathrm{cpm}$. All the observations above point out that the clearance ratio has more significant effects on the natural frequency with small clearance than that with large value.

\section{3) Stability Margin with Varying Centering Spring Stiffness}

The aspect ratio and clearance ratio are fixed to 0.2 and 1/1000, respectively, for all the cases with varying spring stiffness. Figures from 6.24 to 6.27 depict the influence of centering spring stiffness on the stability behavior of the HP rotor. In Figure 6.24 and 6.25, the stiffness changes from $0.5 \mathrm{e} 5 \mathrm{lbf} /$ in to $2.5 \mathrm{e} 5 \mathrm{lbf} / \mathrm{in}$. The figure shows that the natural frequency 


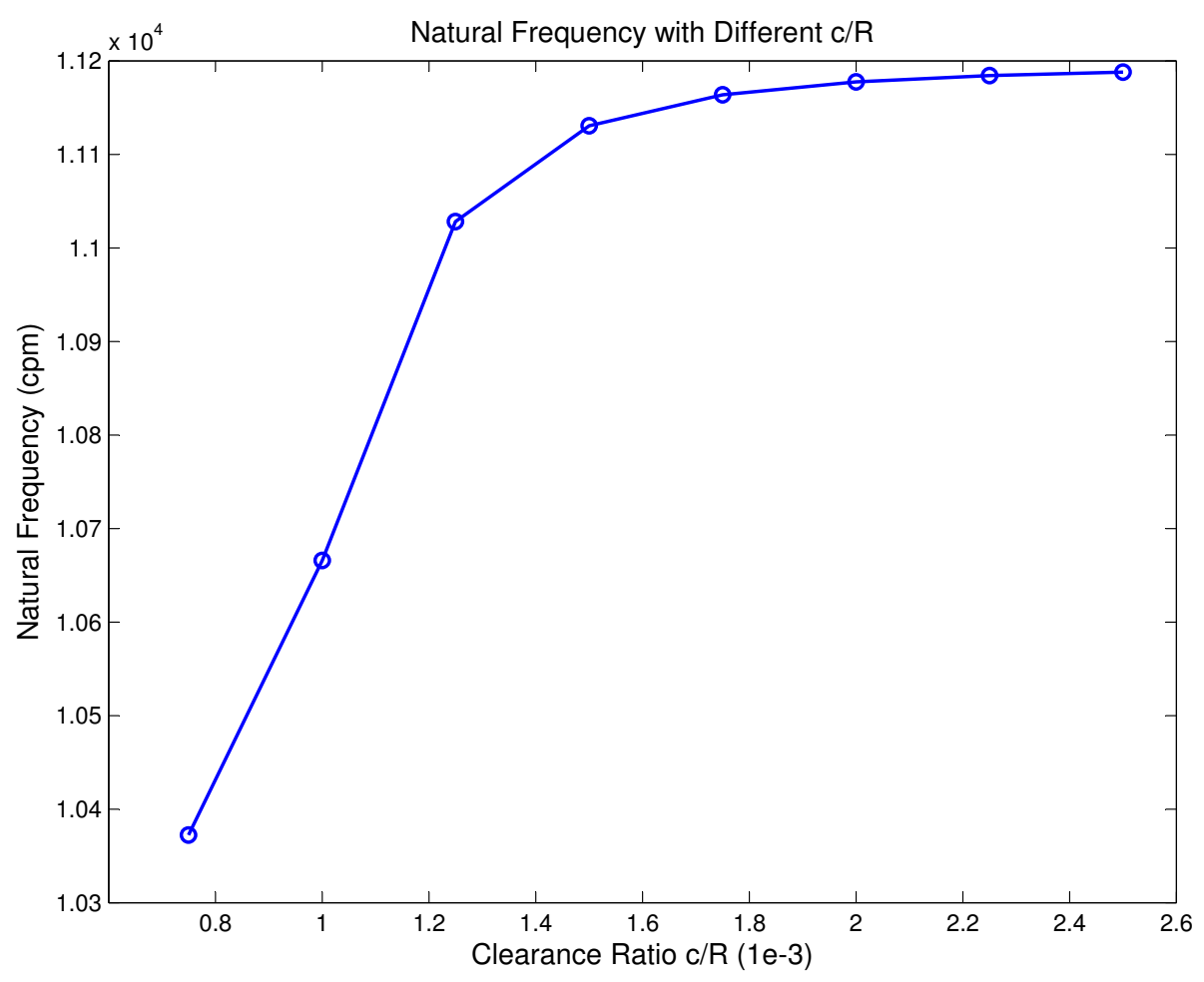

Figure 6.22: Natural Frequency with Varying Clearance Ratio

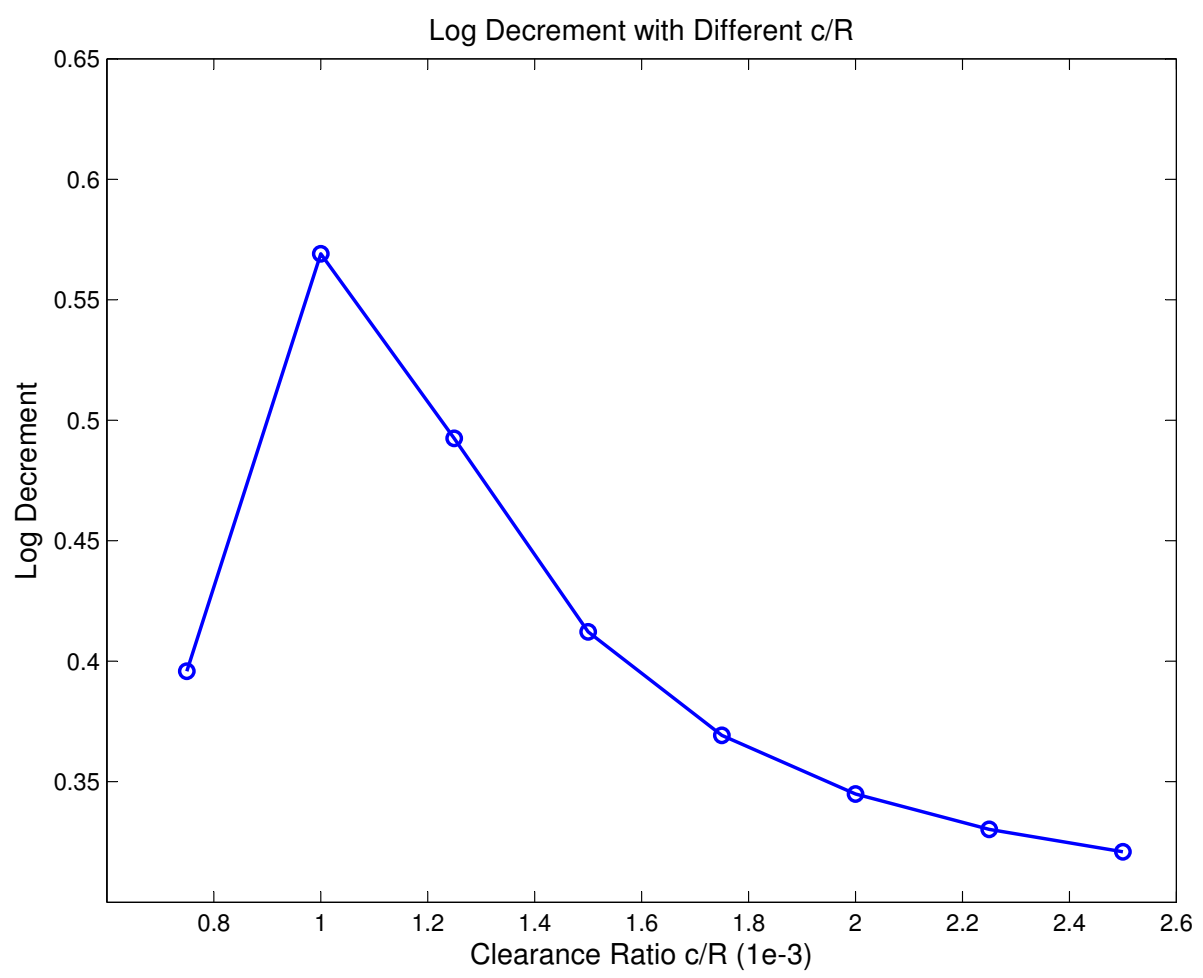

Figure 6.23: Log Decrement with Varying Clearance Ratio 


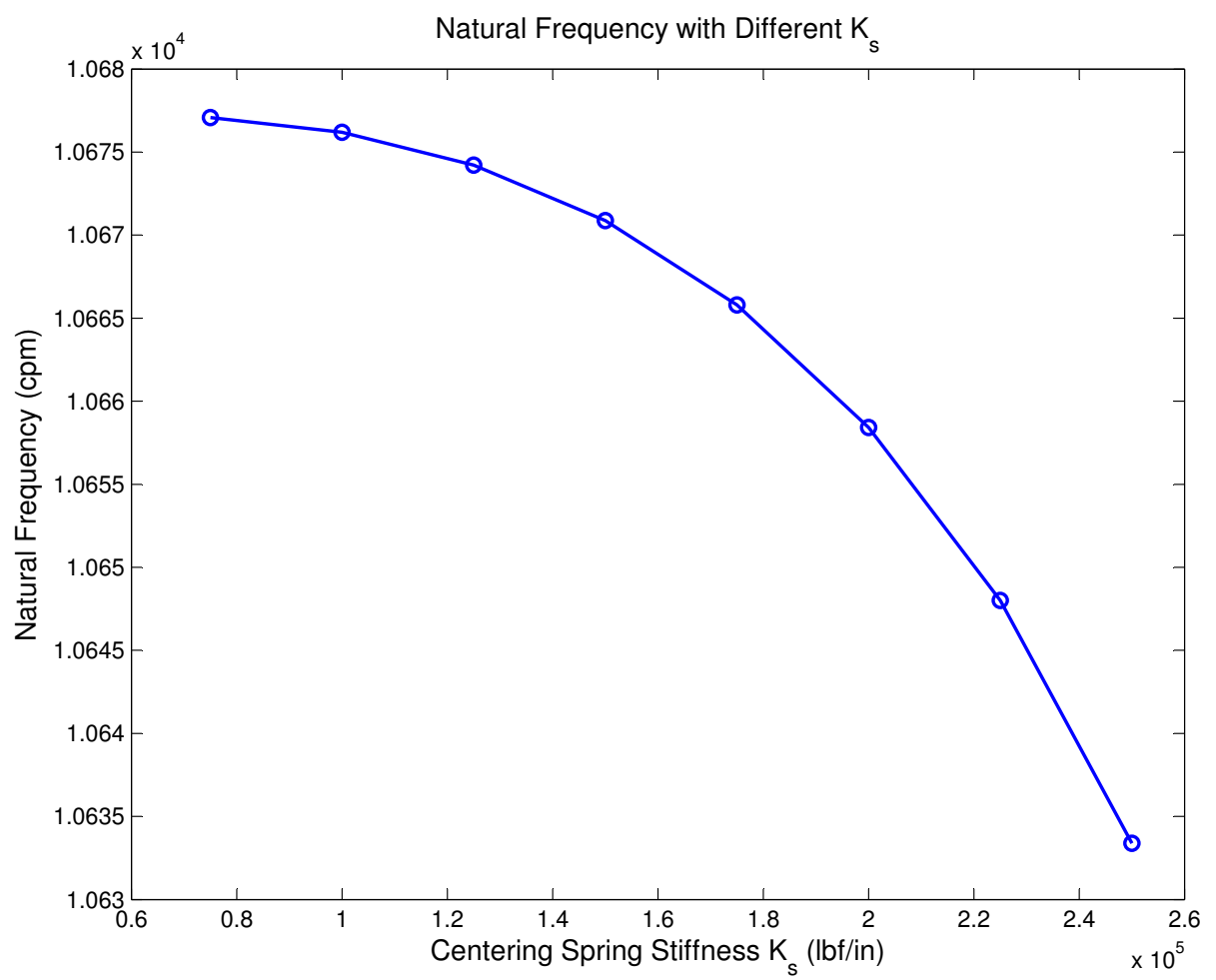

Figure 6.24: Natural Frequency with Varying Stiffness

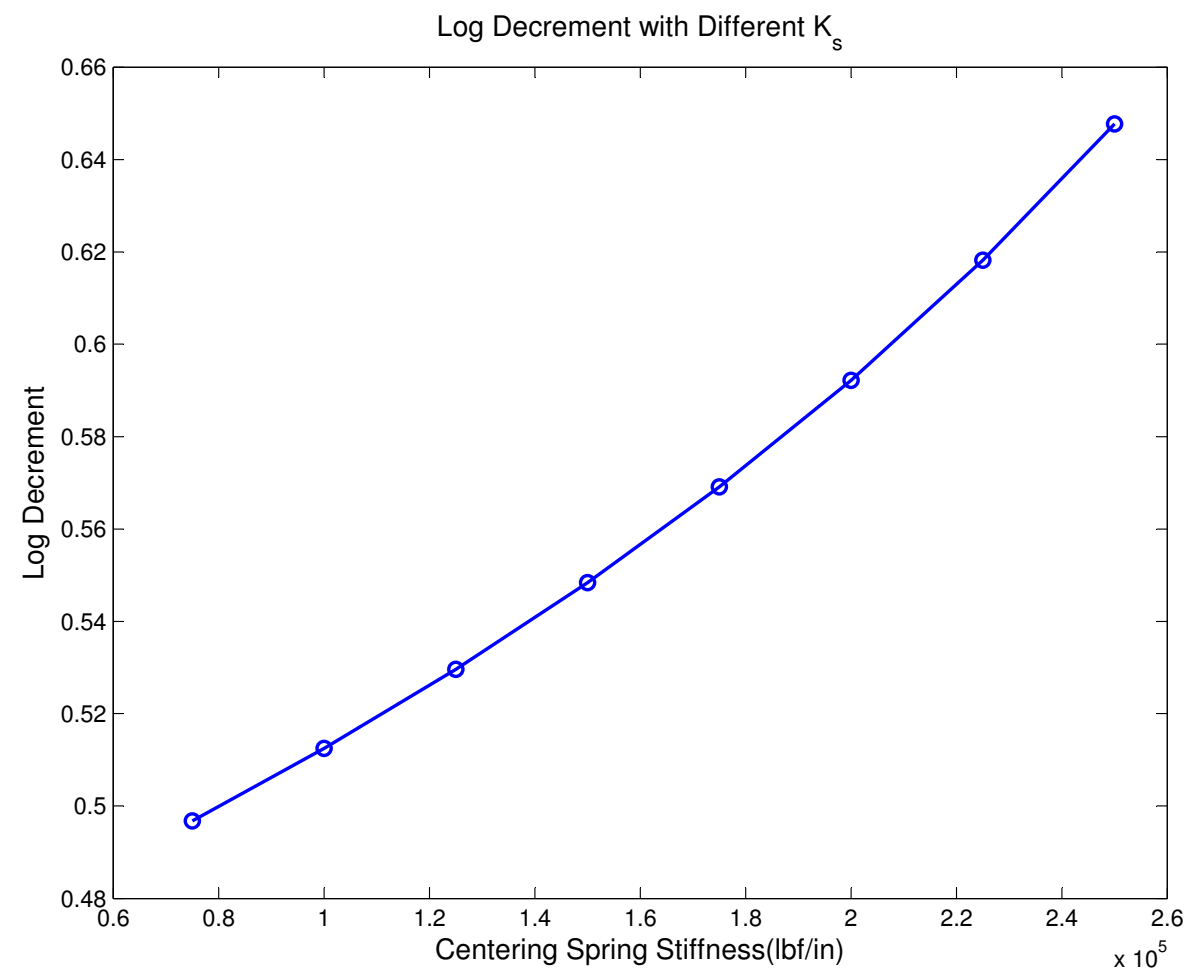

Figure 6.25: Log Decrement with Varying Stiffness 
decreases with the stiffness and the stability margin is improved as well. This might be opposite to the first impression on effects of the stiffness, however, it is the presence of SFD that gives these kinds of behaviors.

As discussed in the forced responses analysis, the journal has slightly larger precessing orbit with stiffer centering spring. Consequently, the larger displacement results in more damping from the damper. Although the stiffness from the damping is also increasing with the displacement, the increasing rate of the damping with eccentricity is larger than that of the stiffness, for the region with small eccentricities. Therefore, the effective damping of the system increases with stiffer spring and gives a more stable system. However, the stiffness range in the previous two figures does not result in significant variation of both natural frequency and logarithmic decrement. The entire changing range of stiffness only gives about $50 \mathrm{cpm}$ variation for the natural frequency and 0.15 for the logarithmic decrement.

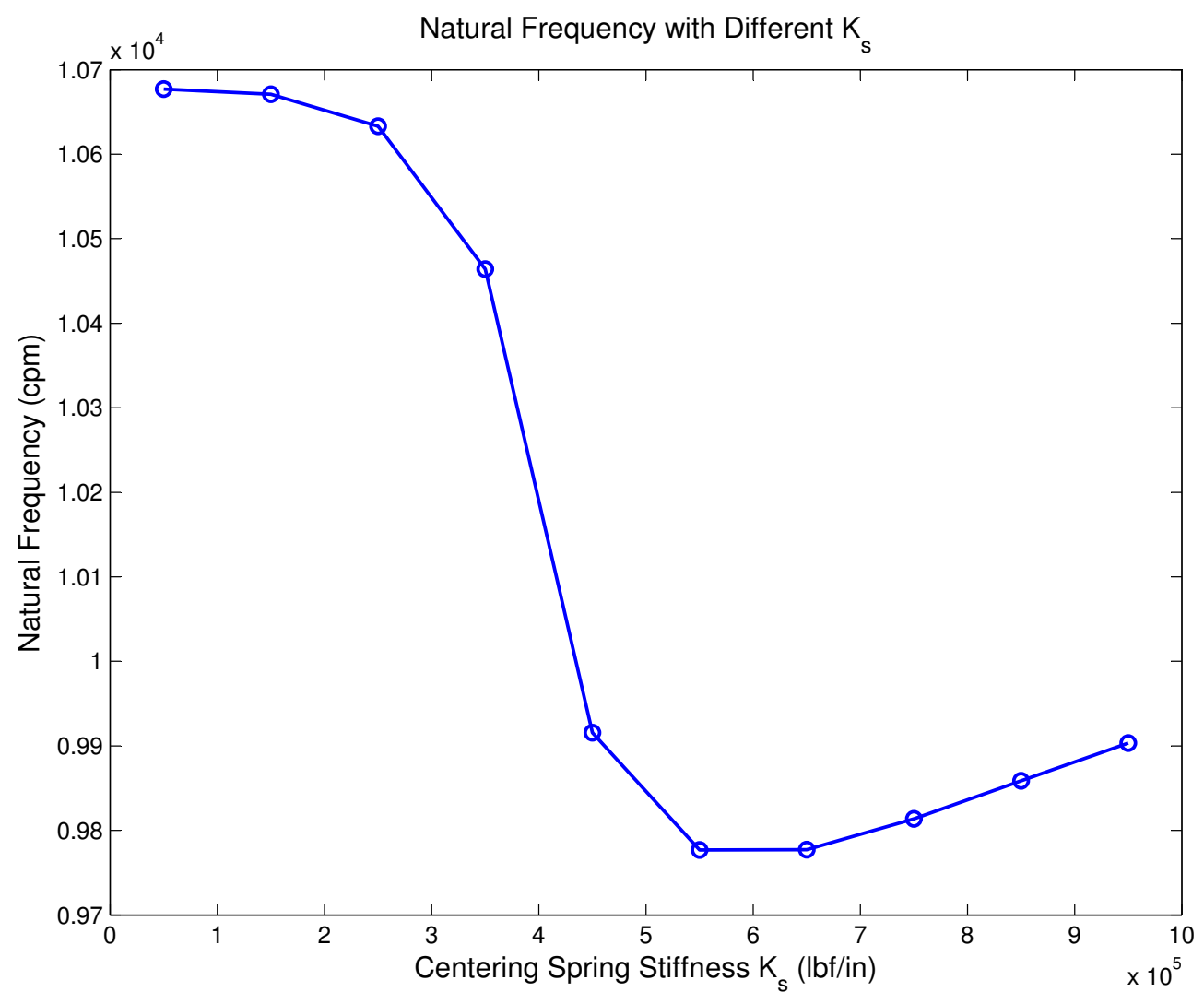

Figure 6.26: Natural Frequency with Varying Stiffness (Wider Range) 
The observations from Figure 6.24 and 6.25 can not be generalized for even larger variation of the centering spring stiffness. Figure 6.26 and 6.27 depict the influence of stiffness with wider variation, from $0.5 \mathrm{e} 5 \mathrm{lbf} /$ in to $9.5 \mathrm{e} 5 \mathrm{lbf} / \mathrm{in}$. Compared with the previous figures, the natural frequency and logarithmic decrement do not change monotonically. The natural frequency starts to increase with spring stiffness as it is larger than $5.5 \mathrm{e} 5 \mathrm{lbf} / \mathrm{in}$, and the logarithmic decrement begins to decrease with large stiffness. The logarithmic decrement reaches its maximum when the stiffness is $4.5 \mathrm{e} 5 \mathrm{lbf} / \mathrm{in}$, which gives the largest stability margin of the system.

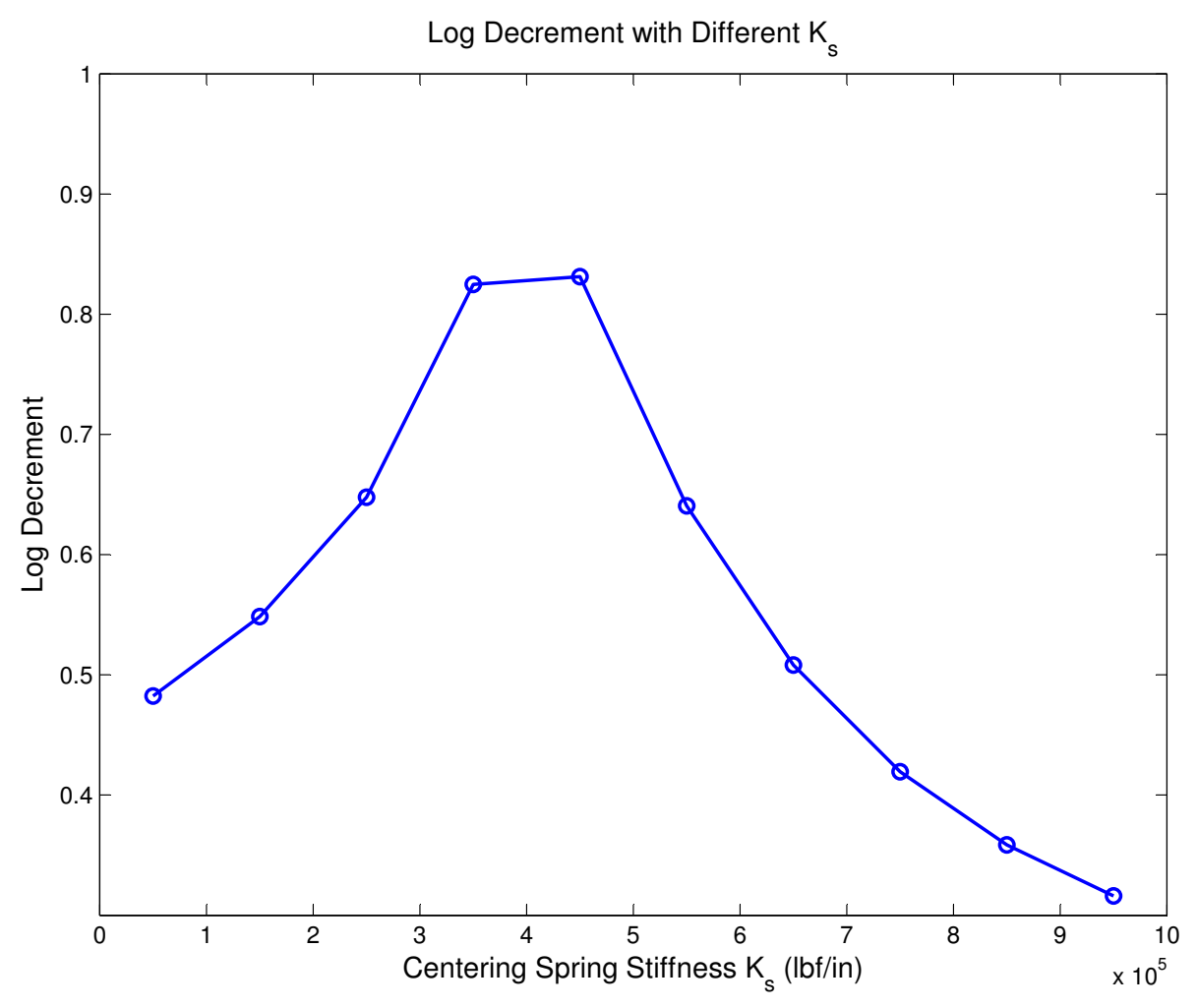

Figure 6.27: Log Decrement with Varying Stiffness (Wider Range)

Figure 6.27 also indicates that, the decreasing rate of the stability margin above $4.5 \mathrm{e} 5$ $\mathrm{lbf} / \mathrm{in}$ is similar to the increasing rate below $3.5 \mathrm{e} 5 \mathrm{lbf} / \mathrm{in}$. The transition range of the spring stiffness between $3.5 \mathrm{e} 5$ and $4.5 \mathrm{e} 5 \mathrm{lbf} / \mathrm{in}$ gives quite flat change. The total difference is less than 0.01, which is negligible, However, the change of the natural frequency within this 
transition range is the most significant, drops from 10,460 cpm to 9,916 cpm, which occupies about $60 \%$ of the entire variation, as shown in Figure 6.26.

The following two sections will study the effects of complicated damper configurations on the rotordynamics of the HP rotor, using the finite difference model of SFDs developed in chapter 3. Two different supply/discharge configurations of SFDs with piston ring seals, central groove and direct hole supply/discharge, will be considered. The aspect ratio $L / D$, clearance ratio $C / R$ and centering spring stiffness are fixed to $0.2,1 / 1000$ and $1.75 \mathrm{e} 5 \mathrm{lbf} / \mathrm{in}$, respectively. The unbalance force is the same as that for the analysis in the previous section using simplified model, with amplitude of $4 W / N$ and phase of $0^{\circ}$ on the second stage of the high pressure turbine. The piston rings for both configurations have the same dimensions, with leakage coefficient $C_{p}=0.5$, ring clearance $h_{s}=C / 2$ and thickness $w=L / 8$. Both two sections focus on the rotordynamics of the rotor at running speed of 15,200 rpm.

\subsubsection{Piston-Ring Seals with Groove Supply/Discharge}

In this section, the effects of SFDs with center groove supply/discharge on the dynamics of the HP rotor are included. The supply and discharge holes are located at $90^{\circ}$ and $270^{\circ}$ in the circumferential direction, respectively. The axial width of the groove is $10 \%$ of damper axial length. The influence of two important factors, the supply pressure and groove depth, on the rotor is documented.

\section{Effects of Supply Pressure}

The variation range of supply pressure is from $1 \mathrm{~atm}$ to $9 \mathrm{~atm}$. The discharge pressure is fixed to $1 \mathrm{~atm}$ for all the cases. The groove depth is $32 C$. The purpose of this deep groove is to make the pressure inside the groove close to the ambient pressure, as shown in Figure 3.25. Figure 6.28 shows the orbits of the journal for all the five cases. The top two orbits near the center of housing gives the comparison between the results from the simplified model in the previous section and the FEA model. The orbit with dotted line inside the blue orbit is the 
one from the simplified model. It is a circular orbit and centered, with about $30 \%$ smaller amplitude than the result from FEA model. Thus, the corresponding damping predicted from the simplified model is under predicted. Note that the comparison is only valid for the supply pressure of $1 \mathrm{~atm}$, since the simplified model is constrained by this assumption. For all other higher supply pressures, the simplified model can not even give close prediction to results from FEA model. Hence, the FEA model is preferred for accurate predictions with supply pressure higher than $1 \mathrm{~atm}$.

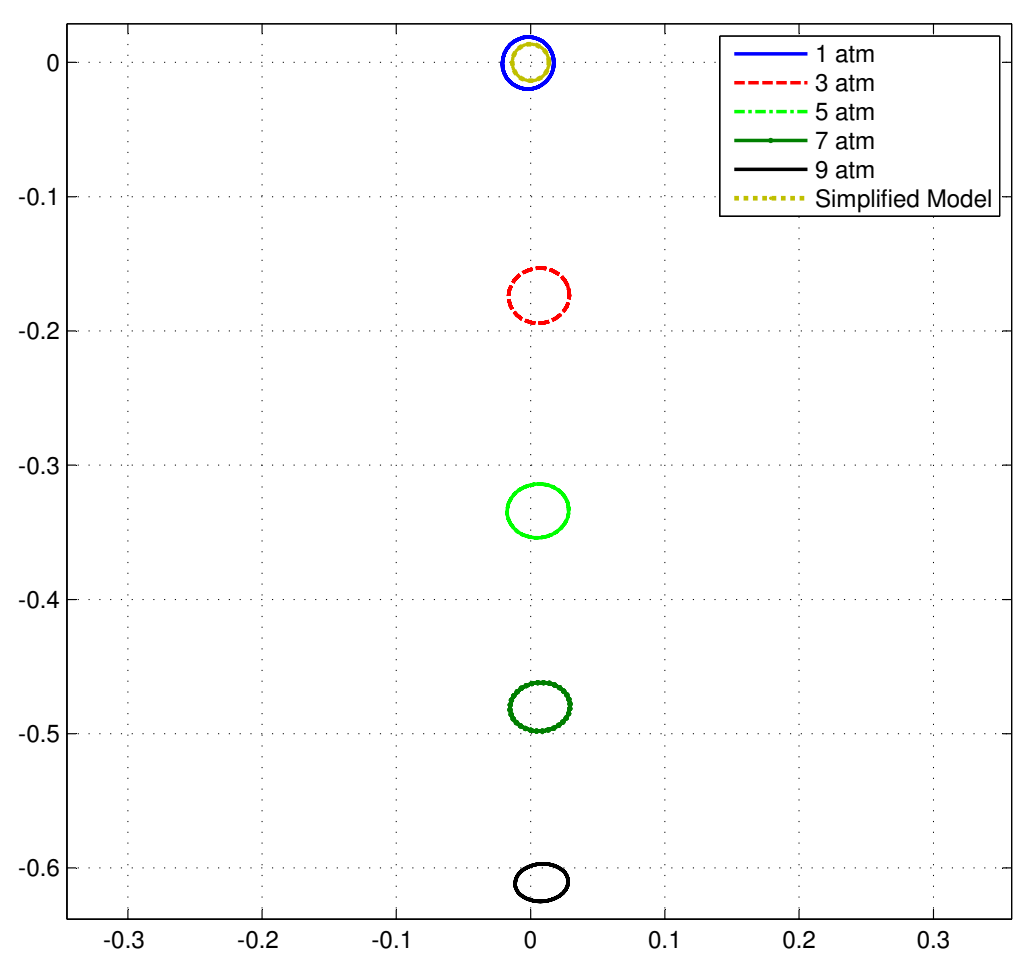

Figure 6.28: Journal Orbits with Supply Pressure(15,200rpm)

As shown in the figure, the main influence of the supply pressure is pushing the journal to more eccentric regions, especially in the vertical direction. The position of orbit center changes from the housing center to the eccentricity ratio of 0.63 . Note that the effects of external loads are not included for all the cases, it is the increase of supply pressure that pushes the orbit center downward. This happens due to the configuration of current damper and the effects of groove. The increasing supply on the top center of the damper lifts the pressure inside the groove, which makes the pressure difference between the supply and 
discharge even larger. Therefore, the net force acting downward on the journal rises with the increasing supply pressure and makes the journal orbits even more asymmetric.

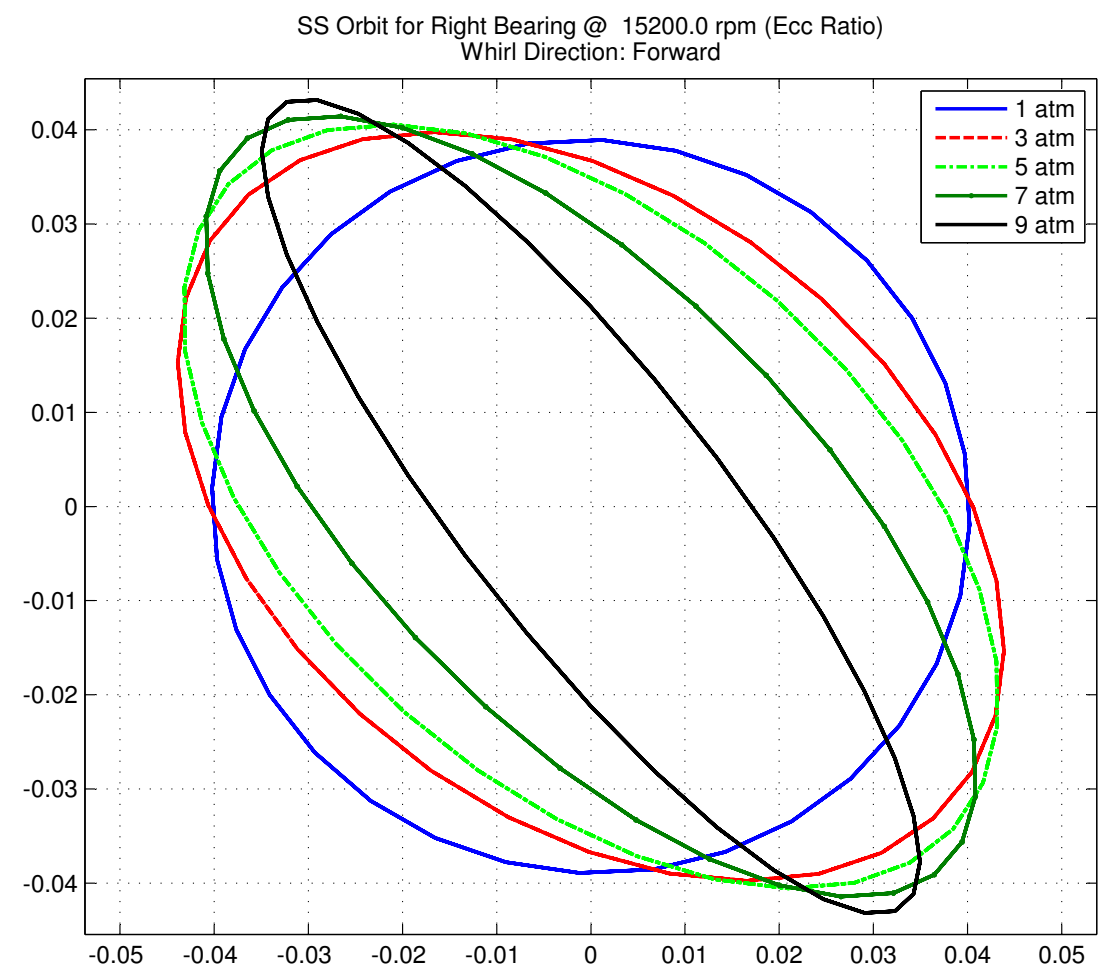

Figure 6.29: Orbits of Right Bearing with Supply Pressure(15,200rpm)

The increase of supply pressure also changes the shape of the orbits. With $p_{s}=1 \mathrm{~atm}$, the orbit is circular and almost centered. This shape turns to ellipse as $p_{s}$ reaches 9 atm, which is more evidence for the asymmetric operation with high supply pressure. This kind of shape change can also be found at other locations of the HP rotor. Figure 6.29 gives the orbits of the node at the location of right bearing, which has more obvious shape variation than the damper. Moreover, all the orbits in Figure 6.29 are centered in the housing. No distinguishable eccentricity is present in either horizontal or vertical direction. This observation indicates the increasing supply pressure does not drive the entire rotor to eccentric operating region, except those near the damper. It is worthy to mention that the orbits ellipticity of the node at the right bearing happens due to the asymmetric support of the SFD at left end, not the symmetric support of the right end. Therefore, the ellipticity of orbits in Figure 6.29 reflects 
the asymmetry of the damper. As the damper has the most eccentric orbit for the supply pressure of $9 \mathrm{~atm}$, the corresponding elliptical orbit at the right end is the narrowest.

The transition of orbit shape in the previous two figures shows another effect of the increasing pressure on the rotordynamics of the HP rotor, the improvement of system stability. As the orbit becomes more elliptical, the forward force and instantaneous velocity in the tangential direction of the orbits is not in-line. Thus, the added destabilizing energy in the forward direction decreases, which increases system stability. In other words, since elliptical orbits are more capable to digest the energy in forward direction than circular orbits [98], the increase of supply pressure diminishes the forward precession and improves system stability. This observation correlates with the discussion in part 3.3.2, which shows the effective damping of SFD with central groove increases with supply pressure.

\section{Effects of Groove Depth}

To study the effects of groove depth on the responses of HP rotor, the supply and discharge pressure are fixed to $7 \mathrm{~atm}$ and $1 \mathrm{~atm}$, respectively. The variation range of the depth is from 1 times to 32 times the radial clearance of the damper. The conditions of the piston ring seal are the same as the previous part. Figure 6.30 shows the orbits for all the five cases at running speed of 15,200 rpm. These orbits are different from each other in both orbit amplitude and shape. The deepest groove gives the most eccentric orbit in the vertical direction. The groove with depth of $8 C$ has the most elliptical orbit. The decrease of groove depth diminishes the effect of the supply pressure and gives more concentric orbit of the journal. If the groove depth equals to the damper clearance, the damper produces a circular orbit near the center of housing and has the minimum amplitude of the responses.

The figure also shows that for the variation range between $16 C$ and $32 C$ gives similar orbits and eccentricities, which indicates this range does not have significant effects on the damper responses. However, the responses becomes much more sensitive to the depth variation below 16C. The orbit with depth of $8 C$ has the eccentricity ratio jumps from 0.45 to 0.35 , and

further decrease of the depth to $1 C$ produces a circular orbit next to the housing center. The 


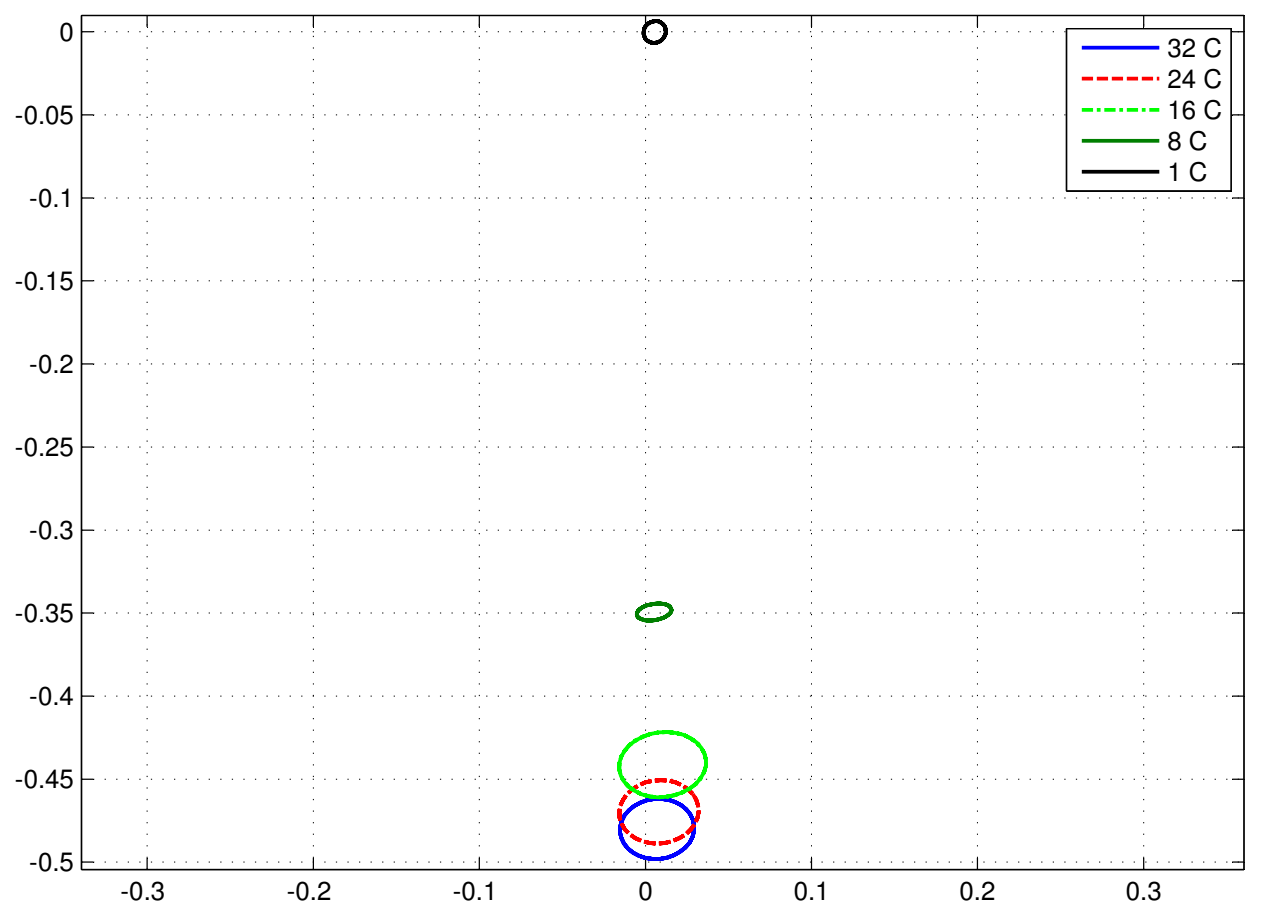

Figure 6.30: Journal Orbits with Groove Depth(15,200rpm)

change of the eccentricity ratio from $8 C$ to $1 C$ is about $35 \%$ of the radial clearance. These significant changes of the eccentricity indicate that caution must be paid to for the range below $16 C$, within which the responses of HP rotor would be quite different with minor depth variation. The above observations have good agreement with the pressure distribution shown in Figure 3.25, which records the sensitive responses of the damper pressure to the groove depth variation under $16 C$.

Figure 6.31 shows the orbits of the rotor at the location of the right bearing. Similar to the orbits in Figure 6.29, the node at the right bearing has centered orbits when subjected to varying groove depth of the left end SFD. The range with depth above $16 C$ gives similar elliptical shape orbits, although the attitude angle of the orbits increases. The orbit ellipticity grows as the groove depth reaches to $8 C$. However, the ellipticity decreases with further depth drops and the orbit becomes circular centered when the depth reaches $1 C$. For the depth of $8 C$, the orbits not only shrink its size but also gives the flattest shape, therefore, it is the most stable among all the five variation cases. 


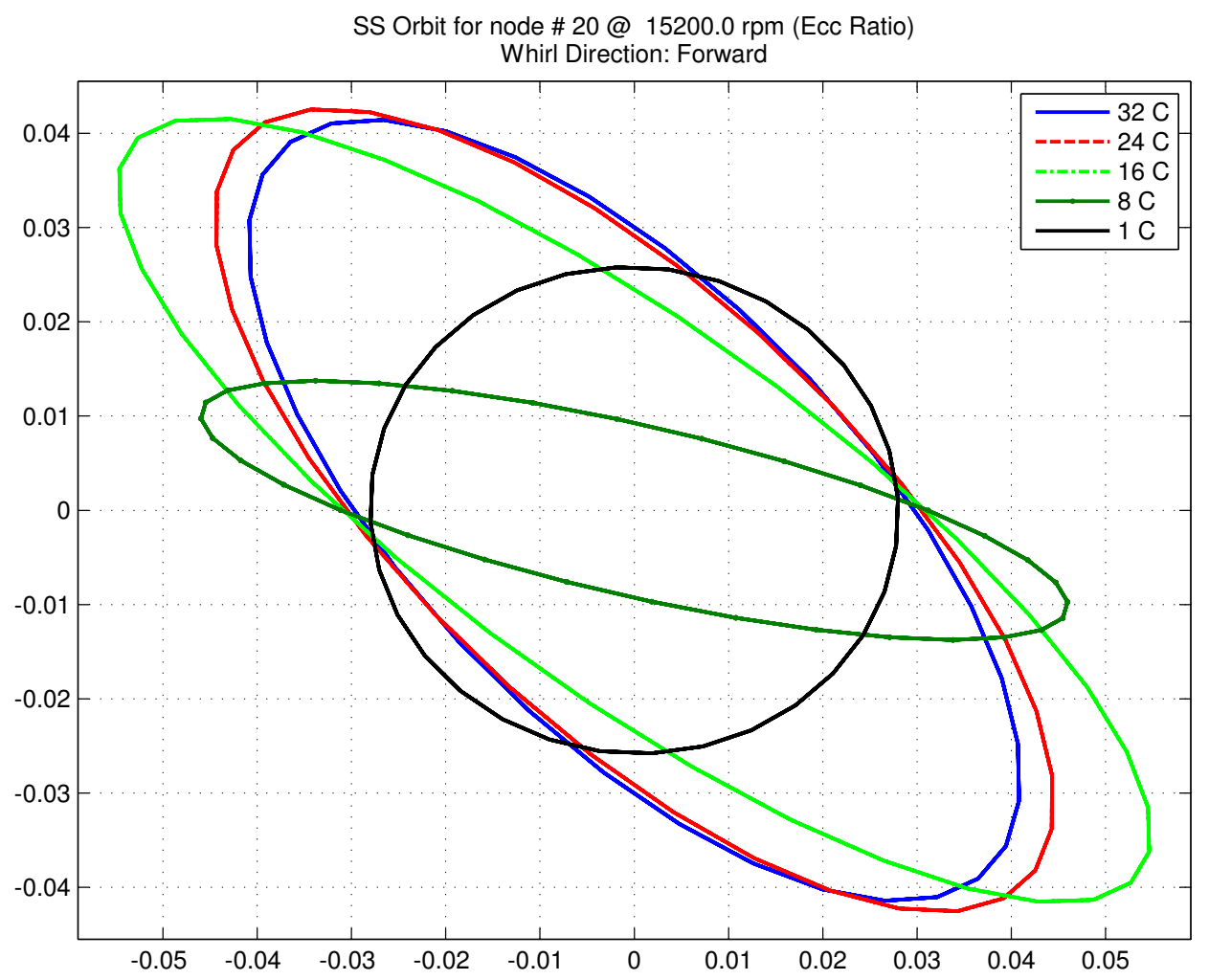

Figure 6.31: Orbits of Right Bearing with Groove Depth(15,200rpm)

\subsubsection{Piston-Ring Seals with Direct Hole Supply/Discharge}

The damper in this section has the same dimensions and operating conditions as the previous section, except there is no groove at the middle of the damper. The supply and discharge holes are located at $90^{\circ}$ and $270^{\circ}$, respectively. Figure 6.32 gives the journal orbits subjects to varying supply pressure from $1 \mathrm{~atm}$ to $9 \mathrm{~atm}$, with discharge pressure fixed to $1 \mathrm{~atm}$. Different from the effects of groove supply/discharge in Figure 6.28, the increase of supply pressure through the hole does not produces significant asymmetry. All five cases have the orbits near the housing center, although they are slightly eccentric in the horizontal direction. Comparing to the effects in Figure 6.28, the orbit amplitude is much smaller. It has the largest peak-to-peak value less than 0.02, about half of that in Figure 6.28. This shows that, with same supply pressure, the damper with hole supply/discharge is more effective on the vibration elimination than that of dampers with central groove.

The shape of most orbits in Figure 6.32 are nearly circular, except the supply pressure 


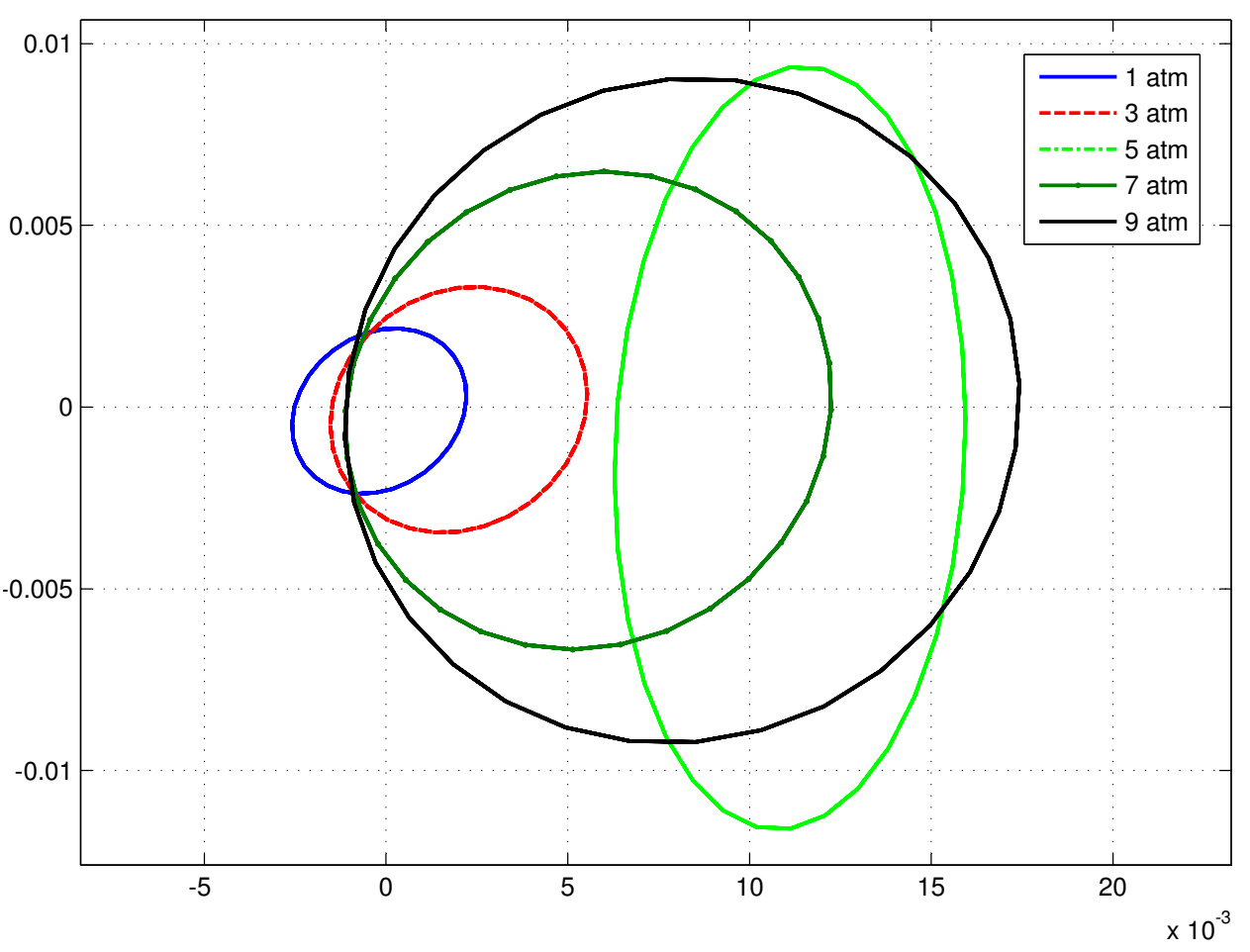

Figure 6.32: Damper Orbits with Supply Pressure (Hole Supply)

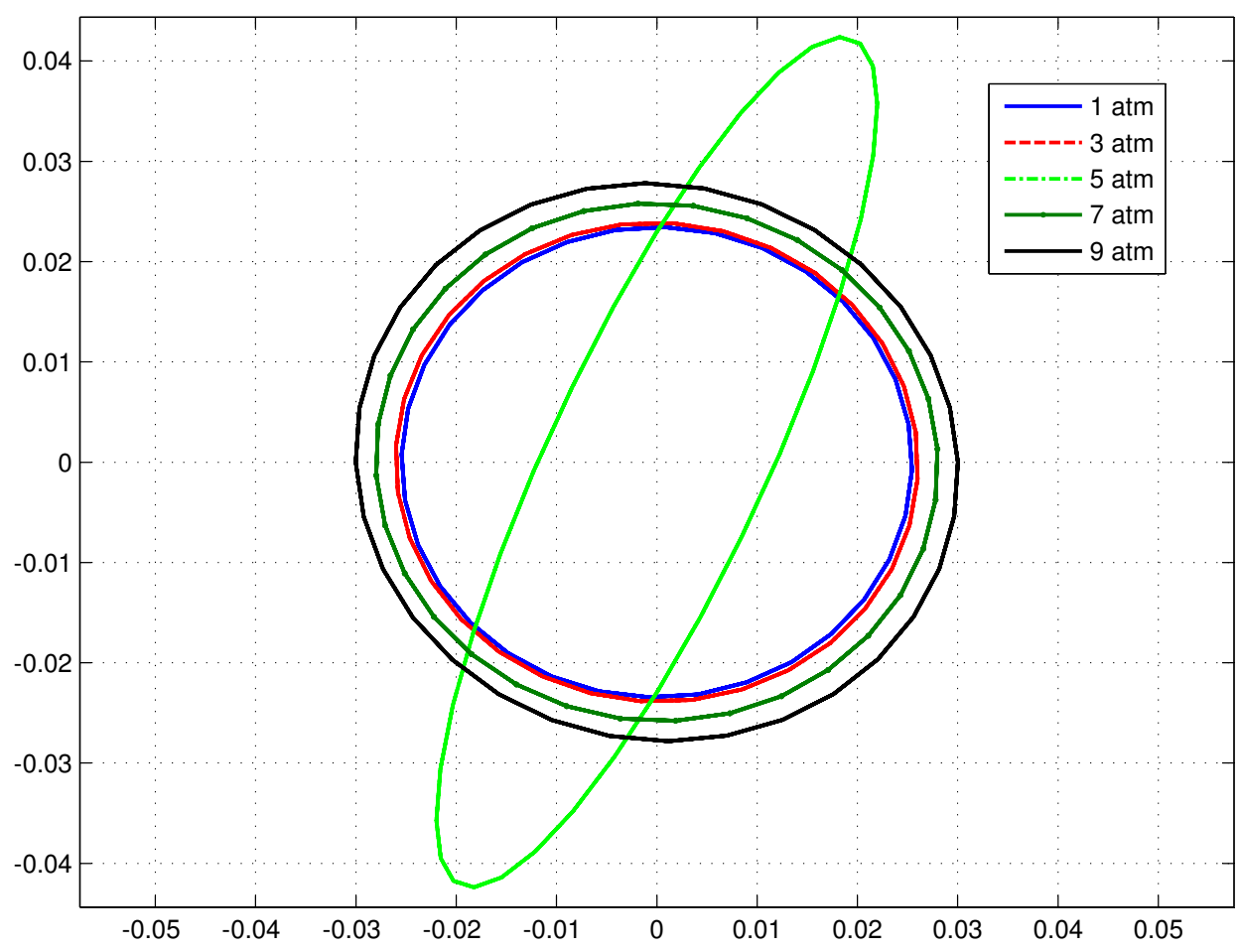

Figure 6.33: Orbits Right Bearing with Supply Pressure (Hole Supply) 
of $5 \mathrm{~atm}$. The $5 \mathrm{~atm}$ case has the most eccentric orbit center and elliptical shape with attitude angle about $90^{\circ}$. Figure 6.33 shows the responses at the location of right end bearing. Different from figure 6.29 and $6.31,4$ out of 5 cases have circular centered orbits. The response with pressure of $1 \mathrm{~atm}$ has almost the same as that of $3 \mathrm{~atm}$. The variation range of all the circular orbits is no larger than 0.01 of damper radial clearance. Corresponding to the response of damper journal at the left end, the supply pressure of $5 \mathrm{~atm}$ generates an elliptical orbit with the maximum peak-to-peak response. From stability point of view, this kind of orbit is preferred in diminishing the destabilizing forces and improving system stability.

\subsection{Summary}

This chapter applied two developed methods, the force coefficients method and improved harmonic balance method, to an aero-engine to demonstrate the effectiveness of these two methods for the rotordynamics analysis of complicated rotating systems with SFDs. The rotordynamics of both Low Pressure and High Pressure rotors were investigated. With the model of short bearing assumption of SFDs, the forced responses of the LP rotor with two dampers were studied with parametric changes. The improved harmonic balance was found to be much more effective than the traditional Runge-Kutta method for the steady sate analysis of complicated rotating systems. For the LP rotor, the parametric study showed that the effect of gravity cannot be ignored, especially for dampers with relative soft centering springs over low speeds. The increment of unbalance on either the fan-end or the turbine-end disk can excite another critical speed near $5000 \mathrm{rpm}$ for this nonlinear rotating system.

The rotordynamics of the HP rotor was investigated for SFDs with piston ring seals. A simplified model of this kind of configuration was used to understand the effects of three parameters, aspect ratio, clearance ratio and centering spring stiffness, on the unbalance

response and stability of the rotating system. All of these parameter variations do not have 
significant effects on the critical speeds of the system, except the aspect ratio and clearance ratio. The stability margin analyses were also included in this chapter. The aspect ratio of 0.2 and clearance ratio of $1 / 1000$ are the optimized value of the range studies, which produce the maximum margin to sustain the destabilizing forces from the compressor. The increasing centering spring stiffness within soft range, below $3.5 \mathrm{e} 5 \mathrm{lbf} / \mathrm{in}$, is observed to improve the stability of the HP rotor.

The effects of SFD with piston seals on the rotordynamics of the HP rotor were analyzed using the finite element model developed in chapter 3. Two supply/discharge formats of the damper, central groove and direct holes, were investigated. Two factors, the supply pressure and groove depth, of the central groove were included. The increasing supply pressure was found to produce more eccentric responses and change the orbit shape from circle to ellipse, which improves the system stability. The decrease of groove depth is able to counter the effects of high supply pressure generating circular centered orbits. Different variation ranges of the depth have different effects on the responses of the HP rotor. The rotor is the most sensitive to the depth change under $16 C$. To reduce the vibration amplitude, the direct hole supply/discharge was found to be more effective than the central groove. The increasing supply pressure through the hole does not give larger responses monotonically. The middle value of supply pressure, $5 \mathrm{~atm}$, gives the highest response amplitude and the most elliptical orbit. 


\section{Chapter 7}

\section{Conclusions and Recommendations}

\subsection{Conclusions}

This study focused on the forced responses and stability of flexible rotor-bearing systems on squeeze film dampers. Specifically, this work aimed at developing effective methods for the rotordynamics analysis of complicated rotor-bearing systems with SFDs, and studying the influence of SFDs with complex configurations on the rotating systems.

In chapter 3, the component level analysis of SFDs with complicated operating condition was done using the finite difference method. The finite difference models for piston ring seals, direct hole supply/discharge and groove supply/dicharge with holes were developed. The pressure distributions of SFDs were obtained using the developed method, and damping coefficients were obtained using a perturbation method. Both pressure distributions and damping coefficients were studied when the damper is subject to parametric changes on these configurations. For the piston ring sealed damper, the parameters contain the leakage coefficients, ring radial clearance and ring axial thickness. The effects of supply pressure and hole numbers on pressure and damping generation were obtained for the direct hole supply. For the grooved damper with hole sypply/discharge, the influence of groove depth and groove supply pressure were also documented for the component level analysis. 
Chapters from chapter 4 to 6 concentrated on the system level analysis of rotor-bearing systems on SFDs. In chapter 4, the forced responses and stability of a flexible system were gained using force coefficients method. To handle dampers with high nonlinearity, the Curve Intersection Method was developed to free the constraints on the traditional iteration method when using the force coefficients of SFDs. The effects of two simplified damper models, the open-end damper with full end leakage and central groove damper with no end leakage, on the unbalance responses and stability of a flexible rotor were discussed. The system behavior is subject to operating conditions of the shaft. The variation of damper dimensions and the consideration of fluid inertia were compared in the chapter.

The effort to remove the assumptions of simplified damper models in the force coefficients method was made in chapter 5. To meet the requirements from industrial applications with complicated SFDs, the harmonic balance method in the frequency domain was improved by introducing the idea of Homotopy and the predictor-corrector procedure. With these enhancements, the effective incorporation of complex dampers becomes possible for the rotordynamic analysis of flexible rotating systems having high order degrees of freedom. The developed method was applied to a flexible rotor-bearing system subjects to different operating conditions, including large unbalance forces, unidirectional loads, stiffness of centering spring and gyroscopic effects. The comparison between the improved harmonic balance method and force coefficient method showed not only good agreement but also the ability of the improved frequency domain method to capture behavior that was missed using the force coefficient method.

The rotordynamic analysis of a complicated rotor-bearing system, an aero-engine, was investigated in chapter 6 , using both the force coefficients method and the improved harmonic balance method. The forced responses of the LP rotor was obtained using the improved frequency domain method. The results were compared with those from the Runge-Kutta method. The effects of two parameters, the unbalance on the rotor and the centering spring stiffness of the damper, on the responses were studied as well. For the HP rotor, the chapter 
focused on the influence of piston ring sealed dampers on the responses and stability. The simplified model of a centrally grooved damper with piston rings was used to study the rotordynamics of the rotor when it is subjected to variations in the damper dimensions. The influence of the cross-coupled forces from the axial compressor on rotor stability margin was also included. For the finite element model, piston ring sealed dampers with both central groove and direct hole supply/discharge were considered.

Based on these efforts, the main conclusions of the work are the following,

\section{Component Level}

\section{a. Damper with Piston Ring}

The three parameters of the piston rings, the axial thickness, ring clearance and leakage coefficient, affect the pressure and damping capacity differently. The change of leakage coefficient has the most influence on both the pressure and damping, while the axial thickness has the least effects. For the leakage coefficient, the variation range above five gives slightly higher pressure than the open end dampers. While the range below one generates significantly higher damping capacities, the coefficient of 0.1 gives more than five times larger pressure than the open end dampers. Therefore, given a SFD with piston ring seals, the benchmark of the leakage coefficient is required in order to give accurate pressure and damping predictions.

\section{b. Damper with Direct Hole Supply/Discharge}

The supply/discharge pressure through the hole dominates the pressure in the axial direction of short damper and generates abnormal pressure at damper end. The smoothness of end pressure recovers for the long dampers, within which the direct hole supply/discharge has limited influence on the pressure at damper ends. The increasing supply pressure does not affect the cross-coupled damping but generates higher direct damping and results in more stable system. Moreover, the variation of supply pressure within low pressure range increases the direct damping more effectively than the pressure change within high range. There is 
an upper limit of supply pressure, above which the damping can not increase further. The effects of supply/discharge pressure of direct hole on the damping capacity also depends on the eccentricity of the journal. The same increment of supply/discharge pressure gives more damping improvement for the operation range with low eccentricity than that with high eccentricity. The numbers of supply/discharge holes also have important influence on the damping generation. Small number of holes(below four) was found to give higher damping forces than large numbers.

\section{c. Damper with Central Groove}

The presence of central groove separates a damper into two film lands generating identical pressure distributions. The pressure inside the groove is not always zero, depending on the groove depth. If the depth is less than 16 times the damper radial clearance, the pressure within the groove has the same order of magnitude as that of the pressure within the surrounding film land. Until the groove is deeper than this range, the pressure inside approaches the ambient pressure. With linear increase of the depth, the maximum damper pressure rises nonlinearly. The pressure is more sensitive to the depth variation below eight times the damper radial clearance than the one above. The increasing groove depth changes not only the maximum pressure inside damper but also the pressure profile. With shallow groove, the maximum damper pressure locates at the middle of the film land. While the deep groove shifts the largest pressure to the location of end seals. For the damping coefficients, the shallowest groove has the largest damping for both cross-coupled and direct terms. However, the changing paths with increasing depth are different for both terms. The depth larger than eight times the damper radial clearance does not have notable influence on the cross-coupled damping. While the direct damping keeps on decreasing until it reaches an asymptotic value, which has the groove pressure equaling to the ambient pressure.

The supply/discharge pressure through the holes of grooved damper is another important factor to the pressure distribution and damping generation. Different from the pressure through direct hole supply/dicharge, the supply/discharge pressure with grooved damper 
gives smooth pressure distribution along the circumferential direction at axial ends. It only affects the pressure profile inside the groove. For the damping coefficient, the increasing supply/discharge pressure has the effects through the elimination of cavitation, especially for the orbits having eccentricity above $50 \%$ of damper clearance. Within this range, the higher supply/discharge pressure gives smaller cross-coupled damping but larger direct damping, which results in more stable systems. For the operating range with low eccentricities, the increasing supply/discharge pressure does not have noticeable influence on the elimination of cavitation. Therefore, both cross-coupled and direct damping with different supply/discharge pressure are close to each other.

\section{System Level}

\section{a. Force Coefficients Method}

The Curved Intersection Method developed in this dissertation is more effective than the traditional iteration procedure when using the force coefficients method to incorporate SFDs for rotordynamics analysis. The code based on this developed method enables the forced responses and stability analysis of rotor-bearing systems with SFDs. The rotordynamics analysis of a flexible rotor demonstrates the ability of SFDs in the elimination of force responses and the improvement of system stability. With the presence of SFD, both the amplitude and amplification factor near the critical speed range reduce significantly, while the critical speed and responses out of this range are similar to the one without SFD. The logarithmic decrement of the studied system increases from 0 to 1.237, if SFD is introduced.

Different from linear rotating systems, the presence of SFD has the responses amplitude increasing nonlinearly with unbalance forces. The larger the unbalance magnitude, the smaller the increase of the amplitude. Additionally, the rising unbalance forces change the amplification factors, which is not the case in linear systems. The higher the unbalance magnitude, the larger the amplification factor around the critical speed, and the smaller the 
logarithmic decrement. This indicates that the large unbalance forces decrease the stability margin of systems with SFDs.

The decrease of damper clearance ratio has similar effects to the increase of aspect ratio on the journal responses. The variation of these two ratios changes the response amplitude, critical speed and amplification factors. For the responses of the journal, the critical speeds decrease with either larger clearance or smaller axial length. The response amplitude has the

reverse trend with the variation of clearance and aspect ratio. Different from the critical speed and response amplitude, the amplification factor does not change monotonically with the two ratios. The factor reaches its minimum value when the clearance ratio and aspect ratio equal to $2 / 1000$ and $1 / 8$, respectively. This finding suggests the optimized stability margin of the rotor-bearing system with SFDs. For the effects of fluid inertia, its consideration differs from the responses of those without inertia only when the running speed is large enough. Meanwhile, the importance of the inertia depends on the damper clearance ratio. The smaller damper clearance, the less effects of the inertia on the responses, even for high operating speed. For a constant speed, the inertia effects improve the stability, especially for the clearance ratio below 5/1000.

\section{b. Direct Force Method}

The direct force method does not have constraints within the force coefficient method and is able to consider dampers with complicated configuration for the rotordynamic analysis. Based on the idea of Homotopy and predictor-corrector procedure, the improved harmonic balance method is effective and efficient to analyze rotating systems having high nonlinearities, including excessive unbalance forces and extreme unidirectional loads. The rotordynamic behavior of flexible rotor-bearing on SFDs is highly dependent on the damper parameters and operating conditions.

The stiffness of damper centering spring affects not only the critical speed and response amplitude but also system stability. The softer the spring stiffness, the smaller the amplification factor, no matter the consideration of external loads. If the gravity is considered, 
the soft spring produces highly eccentric operation and shrinks the orbit size. The excessive unbalance can produce jump responses near critical speeds. Different location and magnitude of unbalance give different jump area (hysteresis loop). The increasing of unidirectional loads generates eccentric orbits, changes the orbit shape from circular to ellipse and introduces super harmonic components to the responses. The gyroscopic effect, which is widely neglected in other analyses, has important influence on the journal responses. The increasing gyroscopic effect at the location away from the damper enlarges both the critical speed and responses amplitude for running speed above the critical. However, for the locations next to the damper, the gyroscopic effect has the opposite influence. The comparison of responses from the force coefficient method and direct force method shows good agreement. The results from these two methods not only have the same critical speeds but also quite similar response amplitude. However, the improved harmonic method is able to capture the elliptical orbits which are missed using the force coefficient method.

\section{c. the Aero-engine}

The rotordynamic analysis of the Low Pressure rotor demonstrates the effectiveness of the improved harmonic balance over the Runge-Kutta method in the time domain. The responses from these two methods have difference no larger than $1 \%$. However, the computation time of the Runge-Kutta method is at least five times as large as that of the improved harmonic balance method. Due to the consideration of gravity, all the orbits of the journals at both turbine-end and fan-end are eccentric. Under one running speed, these two dampers can behave differently from each other. One may have circular orbits while the other is in elliptic orbits having super harmonic components.

The rising unbalance force on either fan disk or turbine disk generates higher critical speeds and increases the amplitude of forced response at both dampers. Besides the increase of critical speed and response amplitude, the excessive unbalance also excites additional critical speed for the response of the journal close to the unbalance location. No matter the location of unbalance forces, the corresponding response of each damper increases non-proportionally 
with linear rise of these forces and grows the amplification factor around the critical speed. The stiffness of centering spring is another important factor on the damper responses of the LP rotor. For the stiffness variation of the fan-end damper, the stiffer spring increases the critical speed and amplitude of the responses for both dampers. However, the variation of spring stiffness at the turbine-end damper has much less influence on the responses of both dampers. The amplitude of responses near the critical speed are similar to each other during the variation.

Based on a simplified model for SFDs with piston ring seals, both the unbalance responses and stability of the High Pressure rotor were investigated when the rotor is subject to the variation of damper aspect ratio, clearance ratio and aerodynamic forces from the axial compressor. The increasing aspect ratio diminishes the unbalance responses while keeping the critical speed unchanged. The amplitude of the first critical speed decreases with aspect ratio so quickly that it disappears as the ratio is over 0.2 . The amplification factor increases monotonically with the aspect ratio for the responses around the first critical speed. However, for the second critical speed within the operating range, the factor increases with the ratio at the beginning and reaches the maximum at 0.2 before decreasing. Hence, the stability margin at 0.2 is the largest over all other aspect ratios.

Subjecting to the variation of clearance ratio, the response of the first and the second critical speed behaves differently. The increasing clearance increases the response amplitude near the first critical region without changing the value of the critical speed. While for the region around the second critical, the largest clearance gives the responses with the minimum amplitude. The amplification factor is the smallest when the clearance ratio is $1 / 1000$ and decreases with the ratio above this value. Different from the effects of both aspect ratio and clearance ratio, the influence of centering spring stiffness below $2.5 \times 10^{5}$ is not crucial to the response of the other two. It has negligible effects on the responses of running speeds below the second critical speed, and slightly increases the responses amplitude at the second critical, which gives higher effective damping and slightly larger stability margin with cross-coupled 
stiffness. However, as the spring stiffness increases further above $2.5 \times 10^{5}$, the reverse effect happens, which increases the response amplitude and deteriorates system stability.

The results from finite element model of SFDs show that the simplified model is inadequate for the central grooved damper with piston ring seals. The simplified model is only comparable with the finite element model when the supply pressure is the ambient pressure and the groove depth is larger than $32 C$. The finite element model finds that the higher the supply pressure within the groove, the more eccentric orbits of the journal. Besides the influence on the eccentricity of the orbit, the shape of the orbit changes from circular to ellipse with increasing supply pressure, and the system stability is improved. The decreasing of groove depth has similar influence on the journal responses as the rising supply pressure. The shallower groove generates more concentric responses. The variation of the depth also changes the orbit shape, which becomes more elliptical with decreasing groove depth above $8 \mathrm{C}$ and backs to circular within further decrease. Different from the journal, the right-end bearing has responses with concentric orbits, no matter the supply pressure and the groove depth.

For the dampers with direct hole supply/discharge, the responses at the location of damper do not have significant eccentricities, compared to those for the grooved damper. The largest eccentricity ratio is about 0.01 , while it is more than 0.45 for the responses of the grooved damper. Another difference from the grooved damper is that the largest supply pressure does not generate the most eccentric orbits. The variation of supply pressure also generates centered response orbits for the node at the right end, and most of them are circular with similar amplitude to each other. However, the supply pressure having the most eccentric orbit at the journal location gives the only case with elliptic response of the right end.

\subsection{Contributions}

For the component level analysis of SFDs, the parametric study of pressure distribution and damping capacity of complicated dampers was enabled using the finite difference method. It 
gives a solid tool for optimized design of complex dampers with practical operating conditions. The effects of end leakage, supply and discharge conditions on the damper behaviors were investigated for the first time. The damper with piston ring seals can generate four times more damping than the open-end dampers, depending on the leakage condition at axial ends. The finite difference method developed also finds that the pressure within the central groove is not always zero, which correlates with the experimental results and suggests the discrepancy of the traditional simplified model of centrally grooved damper. The damping capacity of grooved damper predicted from finite difference method can be more than three time as large as the capacity from the simplified model. Additionally, the performance of SFDs with direct hole supply/discharge, which can not be modeled using the simple model, was evaluated initially with the method developed. The increasing supply pressure was captured to not only diminish the cavitations effects but also improve the effective damping.

For the rotordynamic analysis considering SFDs, this work improved the traditional iteration method based on the force coefficients of simplified damper models. The Curve Intersection Method was introduced, which enables the successful analyses of unbalance responses and stability for rotating systems with extreme operating conditions such as excessive unbalance fores. The method developed also makes effective studies possible on damper parameters including aspect ratio and clearance ratio, and realizes the optimized design of SFDs efficiently.

To realize effective rotordynamic analysis with realistic SFDs, the proposed work focuses on the development of an improved harmonic balance method in the frequency domain. Different from traditional harmonic balance method, the improved method introduces the idea of homotopy and the predictor-corrector procedure. Thus, it enables the effective considerations of extreme conditions such as excessive unbalance forces and unidirectional loads. Since the improved method uses a condensed equation of motion, which separates the linear and nonlinear parts of the system, it realizes the efficient analysis of complex rotating systems with high degree of freedom. Moreover, the use of finite element beam 
model ensures the method to consider important factors conveniently including gyroscopic effects and cross-coupled forces, which are neglected in the traditional method but observed to be important with the improved method.

Using the methods developed by this work, the parametric studies on the unbalance forces and centering spring stiffness of an aero engine are investigated originally. The effects of practical damper configurations with piston ring seals on the forced responses of the engine are discussed for the first time. The results obtained demonstrate the rotordynamic features of the engine with SFDs having central groove supply or direct hole supply, and underline the discrepancy of simplified damper models for the rotordynamic analysis of complex rotating systems on SFDs with practical configurations.

\subsection{Recommendations for Future Work}

The recommendations for future work on both the component level and system level analysis are the following,

For the component level analysis, future work might include the fluid inertia effects of complicated dampers, the establishment of a reliable cavitation model and other end seal conditions such as O-ring. For the fluid inertia effects, though their influence on the performance of simple dampers have been studied by others and part of these results have been applied in this work, the inertia effects within the practical dampers having complicated configurations are not well understood yet. These configurations include end seals conditions, like piston ring seal and O-ring seal, and widely used supply/discharge methods, such as central groove, direct holes. The numerical methods might give the direction to consider the fluid inertia effects within these complicated dampers.

For the consideration of cavitation, this work limits to use $\pi$-film or simplified truncation model. However, the cavitation happening in the field is much more complicated than these two models. Although some efforts have been made to give accurate predictions, their 
comparison with experimental results show no satisfactory correlations. To generate better predictions, it might be necessary to apply multiphase flow theory [126] in future work.

For the consideration of end seals, the proposed work focused on the damper with piston ring seal only. Other sealing conditions such as O-ring seals have the application in rotating systems having light weight. Besides the role of sealing, O-ring seal also acts as a centering spring due to its elastomer properties $[127,128]$. These kind of properties depend on not only the material and dimensions of the ring but also the dynamics of the journal. Hence, the nonlinearities of O-ring sealed dampers come from both the hydrodynamics of the fluid film and these elastomer properties. These two aspects are coupled with each other and provide interesting topics for future work.

For the system level analysis, experimental work to validate the methods developed in this work is essential for future work. Although the predictions from different methods presented in the current work demonstrate good correlation among each other, the experimental validations are necessary to benchmark the prediction tools. Another recommendation for future work is to improve the efficiency of rotordynamic analysis with complicated dampers. Though the methods of current work are more efficient than traditional methods, the efforts using finite element analysis to obtain the dynamic forces of dampers with piston ring seals, central grooves or holes occupy the majority of computation time. To improve this, the study of new algorithms to get the forces of practical dampers more efficiently is recommended for future work. 


\section{References}

[1] E. J. Gunter, L. E. Barrett, and P. E. Allaire. Design and application of squeeze film dampers for turbomachinery stabilization. October 1975.

[2] R. A. Marmol and J. M. Vance. Squeeze film damper characteristics for gas turbine engines. ASME, Transactions, Journal of Mechanical Design, 100(1):139146, 1978.

[3] R. Seydel. Practical bifurcation and stability analysis: from equilibrium to chaos. Springer, 1994.

[4] Rolls-Royce. AE 3007. Technical report, Rolls-Royce plc, 1998.

[5] Luis San Andres. Effect of Fluid Inertia on Squeeze Film Damper Force Response. PhD thesis, Texas A\&M University, United States - Texas, 1985. Ph.D.

[6] F. Y. Zeidan and J. M. Vance. Cavitation regimes in squeeze film dampers and their effect on the pressure distribution. Tribology Transactions, 33(3):447-453, 1990.

[7] K. E. Rouch. Experimental evaluation of squeeze film damper coefficients with frequency domain techniques. Tribology Transactions, 33(1):67-75, 1990.

[8] L. San Andres and D. Lubell. Imbalance response of a test rotor supported on squeeze film dampers. Journal of Engineering for Gas Turbines and Power, 120(2):397, 1998.

[9] G. L. Arauz and L. A. San Andres. Experimental pressures and film forces in a squeeze film damper. Journal of Tribology, 115(1):134, 1993.

[10] G. L. Arauz and L. San Andres. Effect of a circumferential feeding groove on the dynamic force response of a short squeeze film damper. Journal of tribology, 116(2):369$377,1994$.

[11] P. Y. P. Chen and E. J. Hahn. Pressure distribution in squeeze film dampers with oil hole feed. Proceedings of the Institution of Mechanical Engineers, Part J: Journal of Engineering Tribology, 208(2):105-112, June 1994.

[12] J. F. Walton II, J. A. Walowit, E. S. Zorzi, and J. Schrand. Experimental observation of cavitating squeeze-film dampers. Journal of Tribology, 109(2):290-294, 1987.

[13] J. A. Tichy. The effect of fluid inertia in squeeze film damper bearings a heuristic and physical description. In American Society of Mechanical Engineers, International Gas 
Turbine Conference and Exhibit, 28th, Phoenix, AZ; United States; 27-31 Mar. 1983, 1983.

[14] L. San Andres and J. M. Vance. Effects of fluid inertia and turbulence on the force coefficients for squeeze film dampers. Journal of Engineering for Gas Turbines and Power, 108(2):332-339, 1986.

[15] John A. Tichy. Measurements of squeeze-film bearing forces and pressures, including the effect of fluid inertia. A L E Transactions, 28(4):520-526, 1985.

[16] Luis A. San Andres and John M. Vance. Effect of fluid inertia on squeeze-film damper forces for small-amplitude circular-centered motions. ASLE Transactions, 30(1):63-68, 1987.

[17] Stephen Edney and Jhon Nicholas. Retrofitting a large steam turbine with a mechanically centered squeeze film damper. pages 29-40, College Station, TX, 1999.

[18] S. E. Diaz. Orbit-based identification of damping coefficients of off-centered squeeze film damper including support flexibility. ASME Paper, 2000.

[19] R.A Cookson and S.S Kossa. The effectiveness of squeeze-film damper bearings supporting rigid rotors without a centralising spring. International Journal of Mechanical Sciences, 21(11):639-650, 1979.

[20] Q. Li, L. Yan, and J. F. Hamilton. Investigation of the steady-state response of a dual-rotor system with intershaft squeeze film damper. Journal of Engineering for Gas Turbines and Power, 108(4):605-612, October 1986.

[21] J. E. H. Sykes and R. Holrnes. The effects of bearing misalignment on the non-linear vibration of aero-engine RotorDamper assemblies. Proceedings of the Institution of Mechanical Engineers, Part G: Journal of Aerospace Engineering, 204(2):83-99, July 1990.

[22] C.S. Zhu, D. A. Robb, and D. J. Ewins. Analysis of the multiple-solution response of a flexible rotor supported on non-linear squeeze film dampers. Journal of Sound and Vibration, 252(3):389-408, May 2002.

[23] T. N. Shiau and A. N. Jean. Prediction of periodic response of flexible mechanical systems with nonlinear characteristics. Journal of Vibration and Acoustics, 112(4):501507, October 1990.

[24] E. J. Hahn and P. Y. P. Chen. Harmonic balance analysis of general squeeze film damped multidegree-of-freedom rotor bearing systems. Journal of Tribology, 116(3):499-507, July 1994.

[25] P. Bonello, M J Brennan, and R. HOLMES. Non-linear modelling of rotor dynamic systems with squeeze film dampers-an efficient integrated approach. Journal of Sound and Vibration, 249(4):743-773, January 2002. 
[26] C Nataraj and H. D. Nelson. Periodic solutions in rotor dynamic systems with nonlinear supports: a general approach. Journal of vibration and acoustics, 111(2):187$193,1989$.

[27] J. Y. Zhao, I. W. Linnett, and L. J. McLean. Stability and bifurcation of unbalanced response of a squeeze film damped flexible rotor. Journal of Tribology, 116(2):361-368, 1994.

[28] N.S. Feng and E.J. Hahn. Density and viscosity models for two-phase homogeneous hydrodynamic damper fluids. Tribology transactions, 29(3):361, 1986.

[29] D C White. The dynamics of a rigid rotor supported on squeeze film bearings. In Conference on Vibrations in Rotating Systems, London, England; United Kingdom; Feb. ry 14-15 1972, pages 213-229. London, Institution n of Mechanical Engineers, 1972.

[30] S. Cooper. Preliminary investigation of oil films for the control of vibration. Wear, 6(6):496-496, November 1963.

[31] D. Hibner and P. Bansal. Effects of fluid compressibility on viscous damper characteristics. In the Conference on the Stability and Dynamic Response of Rotors with Squeeze Film Dampers, pages 116-132, Charlottesville, Virginia, 1979.

[32] F. Y Zeidan and J. M Vance. Cavitation leading to a two phase fluid in a squeeze film damper. Tribology Transactions, 32(1):100-104, 1989.

[33] Sergio E. Diaz and Luis A. San Andres. Measurements of pressure in a squeeze film damper with an Air/Oil bubbly mixture. Tribology Transactions, 41(2):282, 1998.

[34] S. E. Diaz and L. A. San Andres. Reduction of the dynamic load capacity in a squeeze film damper operating with a bubbly lubricant. Journal of Engineering for Gas Turbines and Power, 121(4):703-709, October 1999.

[35] S. E. Diaz and L. A. San Andres. Air entrainment versus lubricant vaporization in squeeze film dampers: An experimental assessment of their fundamental differences. Journal of Engineering for Gas Turbines and Power, 123(4):871-877, 2001.

[36] Luoyi Tao, Sergio Diaz, Luis San Andres, and K. R. Rajagopal. Analysis of squeeze film dampers operating with bubbly lubricants. Journal of Tribology, 122(1):205-210, January 2000.

[37] Sergio Diaz and Luis San Andres. A model for squeeze film dampers operating with air entrainment and validation with experiments. Journal of Tribology, 123(1):125-133, January 2001.

[38] S. S. Chen, M. W. Wambsganss, and J. A. Jendrzejczyk. Added mass and damping of a vibrating rod in confined viscous fluids. American Society of Mechanical Engineers, June 1976. 
[39] F. Zeidan. Application of squeeze film dampers. volume Vol. 36 of no. 6, pages P50-53, 1995.

[40] J. A. Tichy and M. F. Modest. Squeeze film flow between arbitrary two-dimensional surfaces subject to normal oscillations. Journal of Tribology, 100(3):316-322, July 1978.

[41] L. A. San Andres and J. M. Vance. Effects of fluid inertia on finite-length squeeze-film dampers. A S L E Transactions, 30(3):384-393, 1987.

[42] Luis A. San Andres and John M. Vance. Force coefficients for open-ended squeeze-film dampers executing small-amplitude motions about an off-center equilibrium position. ASLE Transactions, 30(1):69-76, 1987.

[43] J. M. Vance and A.J. Kirton. Experimental measurement of the dynamic force response of a squeeze-film bearing damper. Journal of engineering for industry, 97(4):1282-1290, 1975.

[44] L. A. San Andres, G. Meng, and S. Yoon. Dynamic force response of an open-ended squeeze film damper. Journal of Engineering for Gas Turbines and Power, 115(2):341, 1993.

[45] J. Tichy and B. Bou-Sad. Hydrodynamic lubrication and bearing behavior with impulsive loads. Tribology Transactions, 34(4):505-512, 1991.

[46] J. A. Tichy. A study of the effect of fluid inertia and end leakage in the finite squeeze film damper. Journal of tribology, 109(1):54, 1987.

[47] S. H. Crandall and A. El-Shafei. Momentum and energy approximations for elementary squeeze-film damper flows. ASME Transactions Series E Journal of Applied Mechanics, 60:728-736, September 1993.

[48] J. Zhang, J. Ellis, and J. B. Roberts. Observations on the nonlinear fluid forces in short cylindrical squeeze film dampers. Journal of Tribology, 115(4):692-698, 1993.

[49] L. A. San Andres and J. M. Vance. Experimental measurement of the dynamic pressure distribution in a squeeze-film bearing damper executing circular-centered orbit. Tribology transactions, 30(3):373, 1987.

[50] A. Z. Szeri, A. A. Raimondi, and A. Giron-Duarte. Linear force coefficients for squeeze-film dampers. Journal of Lubrication Technology, 105(3):326, 1983.

[51] F. Y Zeidan, L. A. San Andres, and J. M. Vance. Design and application of squeeze film dampers in rotating machinery. In Proceedings of the 25th Turbomachinery Symposium, pages 169-188, Houston,TX, September 1996.

[52] S. Jger, T. Bruchmller, and A. Albers. Dynamic behaviour and sealing performance of piston rings used in squeeze-film-dampers. Sealing Technology, 2012(11):9-13, November 2012. 
[53] J. B. Roberts, R. Holmes, and P. J. Mason. Estimation of squeeze-film damping and inertial coefficients from experimental free-decay data. Proceedings of the Institution of Mechanical Engineers, Part C: Journal of Mechanical Engineering Science, 200(2):123-133, March 1986.

[54] Luis A. San Andres. Analysis of short squeeze film dampers with a central groove. Journal of Tribology, 114(4):659-664, October 1992.

[55] M. C. Levesley and R. Holmes. The effect of oil supply and sealing arrangements on the performance of squeeze-film dampers: An experimental study. Proceedings of the Institution of Mechanical Engineers, Part J: Journal of Engineering Tribology, 210(4):221-232, December 1996.

[56] Adolfo Delgado and Luis San Andres. A model for improved prediction of force coefficients in grooved squeeze film dampers and oil seal rings. Journal of Tribology, 132(3):032202, 2010.

[57] Fabiano A. Rodrigues, Fabrice Thouverez, and Louis Jezequel. Interaction of squeeze film dampers and hole feed systems and its influence on the dynamics of a jeffcott rotor. International Journal of Rotating Machinery, 10(3):163-174, 2004.

[58] M. D. Rabinowitz and E. J. Hahn. Stability of squeeze-film-damper supported flexible rotors. Journal of Engineering for Power, Transactions ASME, 99(4):545-551, 1977.

[59] A. El-Shafei. Unbalance response of a jeffcott rotor incorporating short squeeze film dampers. Journal of Engineering for Gas Turbines and Power, 112(4):445-453, October 1990.

[60] L. J. McLean and E. J. Hahn. Stability of squeeze film damped multi-mass flexible rotor bearing systems. Journal of Tribology, 107(3):402, 1985.

[61] F. Chu and Z. Zhang. Periodic, quasi-periodic and chaotic vibrations of a rub-impact rotor system supported on oil film bearings. International Journal of Engineering Science, 35(10-11):963-973, August 1997.

[62] H. T Yau, C. K Chen, and C. L Chen. Chaos and bifurcation analysis of a flexible rotor supported by short journal bearings with non-linear suspension. Proceedings of the Institution of Mechanical Engineers, Part C: Journal of Mechanical Engineering Science, 214(7):931947, 2000.

[63] Luis A. San Andres and J. M. Vance. Force coefficients for open-ended squeeze-film dampers executing small-amplitude motions about an off-center equilibrium position. ASLE - Transactions, 30(1):69, 1987.

[64] L. M. Greenhill and H. D. Nelson. Iterative determination of squeeze film damper eccentricity for flexible rotor systems. Journal of mechanical design, 104:334, 1982. 
[65] Bugra H. Ertas, Massimo Camatti, and Gabriele Mariotti. Synchronous response to rotor imbalance using a damped gas bearing. Journal of Engineering for Gas Turbines and Power, 132(3):032501-9, March 2010.

[66] D Taylor and B. R. K. Kumar. Nonlinear response of short squeeze film dampers. Journal of lubrication technology, 102(1):51-58, January 1980.

[67] Q. Li and J. F. Hamilton. Investigation of the transient response of a dual-rotor system with intershaft squeeze-film damper. Journal of Engineering for Gas Turbines and Power, 108(4):613-618, October 1986.

[68] J. I. Inayat-Hussain. Bifurcations of a flexible rotor response in squeeze-film dampers without centering springs. Chaos, Solitons \& Fractals, 24(2):583-596, April 2005.

[69] M. Urabe. Galerkin's procedure for nonlinear periodic systems. Archive for Rational Mechanics and Analysis, 20(2):P120-152, 1965.

[70] J. E. Dennis and Jorge J. More. Quasi-newton methods, motivation and theory. SIAM Review, 19(1):46-89, January 1977.

[71] C. G. Broyden. A class of methods for solving nonlinear simultaneous equations. Mathematics of Computation, 19(92):577-593, October 1965.

[72] Jon Li Hwang and Ting Nung Shiau. An application of the generalized polynomial expansion method to nonlinear rotor bearing systems. Journal of Vibration and Acoustics, 113(3):299-308, July 1991.

[73] T. N. Shiau and J. L. Hwang. Generalized polynomial expansion method for the dynamic analysis of rotor-bearing systems. Journal of Engineering for Gas Turbines and Power, 115(2):209-217, April 1993.

[74] T. N. Shiau, J. L. Hwang, and Y. B. Chang. A study on stability and response analysis of a nonlinear rotor system with mass unbalance and side load. Journal of Engineering for Gas Turbines and Power, 115(2):218-226, April 1993.

[75] E. S. Zorzi and H. D. Nelson. Finite element simulation of rotor-bearing systems with internal damping. Journal of Engineering for Power, 99:71, 1977.

[76] M.D. Rabinowitz. Steady-state performance of squeeze film damper supported flexible rotors. Journal of Engineering for Power, Transactions ASME, 99(4):552-558, 1977.

[77] J. Y. Zhao, I. W. Linnett, and L. J. McLean. Subharmonic and quasi-periodic motions of an eccentric squeeze film damper-mounted rigid rotor. Journal of Vibration and Acoustics, 116(3):357-363, July 1994.

[78] C. S. Hsu. Impulsive parametric excitation: Theory. Journal of Applied Mechanics, 39(2):551-558, June 1972.

[79] S. Mohan and E. J. Hahn. Design of squeeze film damper supports for rigid rotors. ASME Journal of Engineering for Industry, 96(3):976-982, 1974. 
[80] Xuehai Li and Dean L. Taylor. Nonsynchronous motion of squeeze film damper systems. Journal of Tribology, 109(1):169-176, January 1987.

[81] Oscar Pinkus and Beno Sternlicht. Theory of Hydrodynamic Lubrication. New York, McGraw-Hill, 1961.

[82] T. J. Copeland. The design and construction of a circular centered orbit squeeze film damper rotor rig. University of Cincinnati, 1994.

[83] J. Zhang, J. B. Roberts, and J. Ellis. Experimental behavior of a short cylindrical squeeze film damper executing circular centered orbits. Journal of Tribology, 116(3):528, 1994.

[84] Luis San Andres and Adolfo Delgado. Identification of force coefficients in a squeeze film damper with a mechanical end seal - centered circular orbit tests. Journal of Tribology, 129(3):660-668, July 2007.

[85] B. H. Ertas, M. Camatti, and G. Mariotti. Synchronous response to rotor imbalance using a damped gas bearing. pages 725-734. ASME, 2009.

[86] F. He and P. E. Allaire. Forced response of a flexible rotor with squeeze film damper under parametric change. San Antonio, Texas, 2013.

[87] R. L. Ruhl and J. F. Booker. A finite element model for distributed parameter turborotor systems. Journal of Engineering for Industry, 94(1):126-132, February 1972.

[88] T. W. Dimond, A. A. Younan, and P. E. Allaire. Comparison of tilting-pad journal bearing dynamic full coefficient and reduced order models using modal analysis (GT2009-60269). Journal of Vibration and Acoustics, 132(5):051009-051009, August 2010.

[89] H. D. Nelson and J. M. McVaugh. The dynamics of rotor-bearing systems using finite elements. Journal of Engineering for Industry, 98(2):593-600, May 1976.

[90] H. D. Nelson. A finite rotating shaft element using timoshenko beam theory. Journal of Mechanical Design, 102(4):793-803, October 1980.

[91] E. S. Zorzi and H. D. Nelson. The dynamics of rotor-bearing systems with axial TorqueA finite element approach. Journal of Mechanical Design, 102(1):158-161, January 1980.

[92] M. Lalanne and G. Ferraris. Rotordynamics Prediction in Engineering, 2nd Edition. Wiley, 2 edition, January 1998.

[93] Fredric F. Ehrich. Handbook of Rotordynamics. Krieger Publishing Company, 3rd edition, August 2004.

[94] Paul E. Allaire. Basics of the finite element method: solid mechanics, heat transfer, and fluid mechanics. W.C. Brown, June 1985. 
[95] API Standard 684. Rotordynamic tutorial: Lateral critical speeds, unbalance response, stability, train tortionals, and rotor balancing, 2005.

[96] Robley Gordon Kirk, Edgar J. Gunter, University of Virginia Dept of Mechanical Engineering, and Lewis Research Center. Transient journal bearing analysis. National Aeronautics and Space Administration, 1970.

[97] A. Muszynska. Rotordynamics. CRC Press, 1 edition, May 2005.

[98] J. M. Vance, F. Y. Zeidan, and B. Murphy. Machinery Vibration and Rotordynamics. Wiley, May 2010.

[99] Maurice L. Adams. Rotating Machinery Vibration: From Analysis to Troubleshooting, Second Edition. CRC Press, August 2010.

[100] C.-T. Chen. Linear System Theory and Design. Oxford University Press, USA, 3 edition edition, September 1998.

[101] E.A. Memmott. Lateral rotordynamic stability criteria for centrifugal compressors. In the 20th Machinery Dynamics Seminar, pages 6.23-6.32, Quebec City, October 2002.

[102] E. A. Memmott. Application of squeeze-film dampers to a large centrifugal compressor. pages 409-420, Glasgow, UK, 2010.

[103] F. He and P. E Allaire. Unbalance response and stability of a three mass rotor supported on squeeze film damper. In ROMAC Annual Meeting, Charlottesville, Virginia, June 2012.

[104] G. E. Forsythe, M. A. Malcolm, and C. B. Moler. Computer Methods for Mathematical Computations. Prentice Hall Professional Technical Reference, 1977.

[105] N. M. Newmark. A method of computation for structural dynamics. Journal of the Engineering Mechanics Division, 85(3):67-94, July 1959.

[106] Josef Stoer and Roland Bulirsch. Introduction to numerical analysis. Springer-Verlag, 1980.

[107] G. von Groll and D. J. Ewins. The harmonic balance method with arc-length continuation in rotor/stator contact problems. Journal of Sound and Vibration, 241(2):223233, March 2001.

[108] R.A. Cookson and S.S. Kossa. The effectiveness of squeeze-film damper bearings supporting flexible rotors without a centralising spring. International Journal of Mechanical Sciences, 22(5):313-324, 1980.

[109] P Bonello, M.J Brennan, and R Holmes. Modelling the non-linear dynamics of an aero-engine low pressure rotor mounted on squeeze film bearings. 2000 .

[110] Carmen Chicone. Ordinary Differential Equations with Applications. Springer, September 2006. 
[111] Gerald Teschl. Ordinary Differential Equations and Dynamical Systems. American Mathematical Soc., 2012.

[112] J Y Zhao and E J Hahn. Subharmonic, quasiperiodic and chaotic motions of a rigid rotor supported by an eccentric squeeze film damper. ARCHIVE: Proceedings of the Institution of Mechanical Engineers, Part C: Journal of Mechanical Engineering Science 1989-1996 (vols 203-210), 207(63):383-392, June 1993.

[113] Eugene L. Allgower and Kurt Georg. Introduction to numerical continuation methods. SIAM, 2003.

[114] Rdiger Seydel. Practical Bifurcation and Stability Analysis. Springer, February 2010.

[115] Fred T. Krogh. Predictor-corrector methods of high order with improved stability characteristics. J. ACM, 13(3):374385, July 1966.

[116] P. M. Anselone and R. H. Moore. an extension of the newton-kantorovic method for solving nonlinear equations. Technical report, January 1965.

[117] D. W Decker and H. B Keller. Path following near bifurcation. Communications on Pure and Applied Mathematics, 34(2):149-175, March 1981.

[118] Hans D. Mittelmann. A pseudo-arclength continuation method for nonlinear eigenvalue problems. SIAM Journal on Numerical Analysis, 23(5):1007-1016, October 1986.

[119] C. B. Haselgrove. The solution of non-linear equations and of differential equations with two-point boundary conditions. The Computer Journal, 4(3):255-259, January 1961.

[120] F. He and P. E. Allaire. Squeeze film damper effects on unbalance response of flexible rotors: Three mass rotor and jet engine. San Diego, CA, June 2011.

[121] ROMAC. Rotor dynamics analysis using ComboRotor. Technical report, University of Virginia, 2010.

[122] J. S. Alford. Protecting turbomachinery from self-excited rotor whirl. Journal for Engineering for Power, 87(4):333-343, October 1965.

[123] A. F. Storace. A simplified method for predicting the stability of aerodynamically excited turbomachinery. Journal of Turbomachinery, 129(4):724-729, October 2007.

[124] A. Muszynska. Whirl and whipRotor/bearing stability problems. Journal of Sound and Vibration, 110(3):443 - 462, 1986.

[125] Jr Earl Logan. Handbook of Turbomachinery. CRC Press, May 2003.

[126] C. E. Brennen. Fundamentals of Multiphase Flow. Cambridge University Press, April 2005. 
[127] A. J. Smalley, M. S. Darlow, and R. K. Mehta. The dynamic characteristics of o-rings. Journal of Mechanical Design, 100(1):132, 1978.

[128] I. Green and C. English. Analysis of elastomeric o-ring seals in compression using the finite element method. Tribology Transactions, 35(1):83-88, 1992. 


\section{Appendix A: Forced Responses Using Harmonic Balance Method}

With the displacement form shown by equation 5.1 , the velocity $\dot{\mathbf{x}}(t)$ and acceleration $\ddot{\mathbf{x}}(t)$ can be expressed as the following,

$$
\begin{gathered}
\dot{\mathbf{x}}(t)=\omega_{i} \sum_{i=1}^{k}\left[\mathbf{X}_{\mathbf{s i}} \cos \left(\omega_{i} t\right)-\mathbf{X}_{\mathbf{c i}} \sin \left(\omega_{i} t\right)\right] \\
\ddot{\mathbf{x}}(t)=-\omega_{i}^{2} \omega_{i} \sum_{i=1}^{k}\left[\mathbf{X}_{\mathbf{c i}} \cos \left(\omega_{i} t\right)+\mathbf{X}_{\mathbf{s i}} \sin \left(\omega_{i} t\right)\right]
\end{gathered}
$$

Substituting the above two equations and equation 5.1 into the equation of motion (equation 5.2) and separating constant, cosine and sine terms result in,

$$
\begin{aligned}
& {[\mathbf{K}]\left\{\mathbf{X}_{\mathbf{0}}\right\}=\frac{1}{T} \int_{0}^{T} \mathbf{f}(\mathbf{x}) d t} \\
& \left([\mathbf{K}]-\omega_{\mathbf{i}}^{2}[\mathbf{M}]\right)\left\{\mathbf{X}_{\mathbf{c i}}\right\}+\omega_{i}([\mathbf{C}]+\Omega[\mathbf{G}])\left\{\mathbf{X}_{\mathbf{s i}}\right\}=\frac{2}{T} \int_{0}^{T} \mathbf{f}(\mathbf{x}) \cos \left(\omega_{i} t\right) d t \\
& \left([\mathbf{K}]-\omega_{\mathbf{i}}^{2}[\mathbf{M}]\right)\left\{\mathbf{X}_{\mathbf{c i}}\right\}-\omega_{i}([\mathbf{C}]+\Omega[\mathbf{G}])\left\{\mathbf{X}_{\mathbf{s i}}\right\}=\frac{2}{T} \int_{0}^{T} \mathbf{f}(\mathbf{x}) \sin \left(\omega_{i} t\right) d t
\end{aligned}
$$

where, $T$ is the period of response. 
Replacing the right-hand side of the above equation, equation 5.3 can be obtained.

$$
\begin{aligned}
& \widetilde{\mathbf{F}}(\mathbf{X})_{\text {constant }}=\frac{1}{T} \int_{0}^{T} \mathbf{f}(\mathbf{x}) d t \\
& \widetilde{\mathbf{F}}(\mathbf{X})_{\cos }=\frac{2}{T} \int_{0}^{T} \mathbf{f}(\mathbf{x}) \cos \left(\omega_{i} t\right) d t \\
& \widetilde{\mathbf{F}}(\mathbf{X})_{\sin }=\frac{2}{T} \int_{0}^{T} \mathbf{f}(\mathbf{x}) \sin \left(\omega_{i} t\right) d t
\end{aligned}
$$

By introducing the complex vector as defined in equation 5.4, the following equation can be obtained for each frequency,

$$
[\mathbf{P}]_{i}\{\widetilde{\mathbf{R}}\}_{i}=\left\{\widetilde{\mathbf{F}}^{L}\right\}_{i}+\left\{\widetilde{\mathbf{F}}^{N L}\right\}_{i}
$$

with,

$$
[\mathbf{P}]_{i}=\left([\mathbf{K}]_{i}-\omega_{i}^{2}[\mathbf{M}]_{i}\right)-j \omega_{i}[\mathbf{C}]_{i}
$$

and,

$$
\begin{aligned}
\left\{\widetilde{\mathbf{F}}^{L}\right\}_{i} & =T_{L} \int_{0}^{T} \mathbf{f}^{L}(\mathbf{x}) e^{j \omega_{i} t} d t \\
\left\{\widetilde{\mathbf{F}}^{N L}\right\}_{i} & =T_{L} \int_{0}^{T} \mathbf{f}^{N L}(\mathbf{x}) e^{j \omega_{i} t} d t \\
T_{L} & = \begin{cases}\frac{1}{T} & i=0, \\
\frac{2}{T} & i=1, \ldots, k .\end{cases}
\end{aligned}
$$

Applying the separation of linear and nonlinear parts, equation 5.5, which is repeated as below, can be result from equation (5).

$$
\left[\begin{array}{ll}
\mathbf{P}_{\mathbf{1 1}} & \mathbf{P}_{\mathbf{1 2}} \\
\mathbf{P}_{\mathbf{2 1}} & \mathbf{P}_{\mathbf{2 2}}
\end{array}\right]_{i}\left\{\begin{array}{c}
\widetilde{\mathbf{R}}_{1} \\
\widetilde{\mathbf{R}}_{2}
\end{array}\right\}_{i}=\left\{\begin{array}{c}
\widetilde{\mathbf{F}}_{1}^{L} \\
\widetilde{\mathbf{F}}_{2}^{L}
\end{array}\right\}_{i}+\left\{\begin{array}{c}
\mathbf{0} \\
\widetilde{\mathbf{F}}_{2}^{N L}
\end{array}\right\}_{i}
$$


Using the first sub-equation of the above expression to represent $\widetilde{\mathbf{R}}_{1}$ in terms of $\widetilde{\mathbf{R}}_{2}$ gives,

$$
\widetilde{\mathbf{R}}_{1}=\left[\mathbf{P}_{11}\right]_{i}^{-1}\left(\left\{\mathbf{F}_{1}^{L}\right\}_{i}-\left[\mathbf{P}_{12}\right]_{i}\left\{\widetilde{\mathbf{R}}_{2}\right\}_{i}\right)
$$

The substitution of the equation above into the second sub-equation of equation (9) result in the objective equation to solve $\widetilde{\mathbf{R}}_{2}$ as bellow,

$$
\left\{\widetilde{\mathbf{R}}_{2}\right\}_{i}=[\mathbf{U}]_{i}^{-1}\left(\left\{\widetilde{\mathbf{F}}_{2}^{N L}\right\}_{i}-\{\mathbf{V}\}_{i}\right)
$$

with $\mathbf{U}$ and $\mathbf{V}$ given as,

$$
\begin{aligned}
& {[\mathbf{U}]=-\left[\mathbf{P}_{\mathbf{2 1}}\right]\left[\mathbf{P}_{11}\right]^{-1}\left[\mathbf{P}_{12}\right]+\left[\mathbf{P}_{\mathbf{2 2}}\right]} \\
& \{\mathbf{V}\}=\left[\mathbf{P}_{\mathbf{2 1}}\right]\left[\mathbf{P}_{11}\right]^{-1}\left\{\mathbf{F}_{\mathbf{L}}^{\mathbf{1}}\right\}-\left\{\mathbf{F}_{\mathbf{L}}^{\mathbf{2}}\right\}
\end{aligned}
$$

Once the displacement of the nonlinear parts $\widetilde{\mathbf{R}}_{2}$ is obtained using equation (11), the responses of the linear parts can be solved with equation (10).

As mentioned in section 5.1.1, equation (11) is an implicit equation, which needs to be solved iteratively. To apply iteration method, such as Newton-Raphson method, the objective equation for $\widetilde{\mathbf{R}}_{2}$ can be transferred to a variant form as,

$$
\overline{\mathbf{Q}}\left(\widetilde{\mathbf{R}}_{2}\right):=\left(\left\{\widetilde{\mathbf{F}}_{2}^{N L}\right\}_{i}-\{\mathbf{V}\}_{i}\right)-[\mathbf{U}]_{i}\left\{\widetilde{\mathbf{R}}_{2}\right\}_{i}
$$

Given the initial guess $\widetilde{\mathbf{R}}_{2}^{i}$, the approximated variant $\overline{\mathbf{Q}}$ of next iteration step is,

$$
\overline{\mathbf{Q}}\left(\widetilde{\mathbf{R}}_{2}^{i}\right)+\left(\frac{d \overline{\mathbf{Q}}}{d \widetilde{\mathbf{R}}_{2}}\right) \Delta \widetilde{\mathbf{R}}_{2} \approx 0
$$

where, $\frac{d \overline{\mathbf{Q}}}{d \widetilde{\mathbf{R}}_{2}}$ is the Jacobian matrix evaluated when $\widetilde{\mathbf{R}}_{2}=\widetilde{\mathbf{R}}_{2}^{i}$. 
Thus, the approximated solution of next step $\widetilde{\mathbf{R}}_{2}^{i+1}$ can be obtained as,

$$
\widetilde{\mathbf{R}}_{2}^{i+1}=\widetilde{\mathbf{R}}_{2}^{i}-\left(\frac{d \overline{\mathbf{Q}}}{d \widetilde{\mathbf{R}}_{2}}\right)^{-1} \overline{\mathbf{Q}}\left(\widetilde{\mathbf{R}}_{2}^{i}\right)
$$

From equation (14), the Jacobian matrix at each iteration step is available using,

$$
\frac{d \overline{\mathbf{Q}}}{d \widetilde{\mathbf{R}}_{2}}=\frac{d \widetilde{\mathbf{F}}_{2}^{N L}}{d \widetilde{\mathbf{R}}_{2}}-[\mathbf{U}]
$$

where, the derivative $\frac{d \widetilde{\mathbf{F}}_{2}^{N L}}{d \widetilde{\mathbf{R}}_{2}}$ can be solved numerically using perturbation method.

Therefore, the procedure from equation (14) to (16) is repeated until $\widetilde{\mathbf{R}}_{2}^{i+1}$ ensures the variant equation (14) to satisfy the given error requirement. 


\section{Appendix B: Stability Using Harmonic Balance Method}

From equation 5.10 to 5.12 , the velocity and acceleration of the perturbed component can be expressed as,

$$
\begin{gathered}
\Delta \dot{\mathbf{x}}(t)=\lambda e^{\lambda t} \mathbf{q}(t)+\omega_{i} e^{\lambda t} \sum_{i=1}^{k}\left(\mathbf{Q}_{\mathbf{s i}} \cos \left(\omega_{i} t\right)-\mathbf{Q}_{\mathbf{c i}} \sin \left(\omega_{i} t\right)\right) \\
\Delta \ddot{\mathbf{x}}(t)=\lambda^{2} e^{\lambda t} \mathbf{q}(t)+2 \lambda e^{\lambda t} \dot{\mathbf{q}}(t)-\omega_{i}^{2} e^{\lambda t} \sum_{i=1}^{k}\left(\mathbf{Q}_{\mathbf{c i}} \cos \left(\omega_{i} t\right)+\mathbf{Q}_{\mathbf{s i}} \sin \left(\omega_{i} t\right)\right)
\end{gathered}
$$

Substituting above two equations in to the equation of motion (equation 5.2) and separating cosine and sine terms gives,

$$
\begin{aligned}
& {\left[\mathbf{K}-\omega_{i}^{2} \mathbf{M}\right]\left\{\mathbf{X}_{c i}\right\}+\omega_{i} \mathbf{C}\left\{\mathbf{X}_{s i}\right\}+\left[\left(\lambda^{2}-\omega_{i}^{2}\right) \mathbf{M}+\lambda \mathbf{C}+\mathbf{K}\right] e^{\lambda t} \mathbf{Q}_{c i}+\left[2 \lambda \omega_{i} \mathbf{M}+\omega_{i} \mathbf{C}\right] e^{\lambda t} \mathbf{Q}_{s i}=\widehat{\mathbf{F}}(\mathbf{X})_{\cos }} \\
& {\left[\mathbf{K}-\omega_{i}^{2} \mathbf{M}\right]\left\{\mathbf{X}_{s i}\right\}-\omega_{i} \mathbf{C}\left\{\mathbf{X}_{c i}\right\}+\left[\left(\lambda^{2}-\omega_{i}^{2}\right) \mathbf{M}+\lambda \mathbf{C}+\mathbf{K}\right] e^{\lambda t} \mathbf{Q}_{s i}-\left[2 \lambda \omega_{i} \mathbf{M}+\omega_{i} \mathbf{C}\right] e^{\lambda t} \mathbf{Q}_{c i}=\widehat{\mathbf{F}}(\mathbf{X})_{\sin }}
\end{aligned}
$$

Using the complex displacement vector $\widetilde{\mathbf{R}}$ and introducing its increment $\Delta \widetilde{\mathbf{R}}$ as,

$$
\Delta \widetilde{\mathbf{R}}=e^{\lambda t} Q_{c i}+j e^{\lambda t} Q_{s i}
$$

the equation (20) can be written as, 


$$
\left(\begin{array}{ll}
\widetilde{\mathbf{K}} & \mathbf{K}^{*}
\end{array}\right)\left[\begin{array}{c}
\widetilde{\mathbf{R}} \\
\Delta \widetilde{\mathbf{R}}
\end{array}\right]=\widetilde{\mathbf{F}}=\widehat{\mathbf{F}}(\mathbf{X})_{\cos }+j \widehat{\mathbf{F}}(\mathbf{X})_{\sin }
$$

where, $\widetilde{\mathbf{K}}$ and $\mathbf{K}^{*}$ are defined by equation 5.14 , which are repeated as following,

$$
\begin{aligned}
& \widetilde{\mathbf{K}}=\mathbf{K}-\omega_{i}^{2} \mathbf{M}-j \omega_{i} \mathbf{C} \\
& \mathbf{K}^{*}=\lambda^{2} \mathbf{M}+\lambda \mathbf{C}-j 2 \lambda \omega_{i} \mathbf{M}+\widetilde{\mathbf{K}}
\end{aligned}
$$

Defining a variant $\mathbf{E}(\widetilde{\mathbf{R}})$ as,

$$
\mathbf{E}(\widetilde{\mathbf{R}}):=\widehat{\mathbf{F}}-\widetilde{\mathbf{K}} \widetilde{\mathbf{R}}
$$

and its Taylor expansion can be expressed as,

$$
\mathbf{E}(\widetilde{\mathbf{R}})=\mathbf{E}\left(\widetilde{\mathbf{R}}_{0}\right)+\frac{\partial \mathbf{E}}{\partial \widetilde{\mathbf{R}}}\left(\widetilde{\mathbf{R}}-\widetilde{\mathbf{R}}_{0}\right)+O\left(\Delta \widetilde{\mathbf{R}}^{2}\right)
$$

Neglecting the high order term $O\left(\Delta \widetilde{\mathbf{R}}^{2}\right)$ and combining the above equation with equation (22) result in,

$$
\left(\mathbf{K}^{*}-\frac{\partial \mathbf{E}}{\partial \widetilde{\mathbf{R}}}\right) \Delta \widetilde{\mathbf{R}} \approx 0
$$

which can be expanded as the following,

$$
\left[\lambda^{2} \mathbf{M}+\lambda\left(\mathbf{C}-j 2 \omega_{i} \mathbf{M}\right)+\widetilde{\mathbf{K}}-\frac{\partial \mathbf{E}}{\partial \widetilde{\mathbf{R}}}\right] \Delta \widetilde{\mathbf{R}} \approx 0
$$

Applying the definition of $\Delta \widetilde{\mathbf{R}}$, the equation above can be transferred to the equation in state space form as given by equation 5.16 , which is repeated as following,

$$
\left[\begin{array}{cc}
\mathbf{M} & \mathbf{0} \\
\mathbf{0} & \mathbf{M}
\end{array}\right]\{\dot{\mathbf{v}}\}+\left[\begin{array}{cc}
\mathbf{0} & -\mathbf{M} \\
\mathbf{K}_{\mathbf{E}} & \mathbf{C}_{\mathbf{E}}
\end{array}\right]\{\mathbf{v}\}=\left[\begin{array}{l}
0 \\
0
\end{array}\right]
$$


The above equation can be represented as standard eigenvalue problem,

$$
\lambda\left[\begin{array}{cc}
\mathrm{M} & \mathbf{0} \\
\mathbf{0} & \mathrm{M}
\end{array}\right]+\left[\begin{array}{cc}
\mathbf{0} & -\mathrm{M} \\
\mathrm{K}_{\mathbf{E}} & \mathrm{C}_{\mathbf{E}}
\end{array}\right]=\mathbf{0}
$$

Therefore, standard eigen solver can be applied to determine the system stability, using the equation above. 